\title{
TRANSCENDING MODERNITY WITH RELATIONAL THINKING
}

Pierpaolo Donati 


\section{Transcending Modernity with Relational Thinking}

This book explores the ways in which social relations are profoundly changing modern society, arguing that, constituting a reality of their own, social relations will ultimately lead to a new form of society: an aftermodern or relational society. Drawing on the thought of Simmel, it extends the idea that society consists essentially of social relations, in order to make sense of the operation of dichotomous forces in society and to examine the emergence of a "third" in the morphogenetic processes. Through a realist and critical relational sociology, which allows for the fact that human beings are both internal and external to social relations, and therefore to society, the author shows how we are moving towards a new, trans-modern society - one that calls into question the guiding ideas of Western modernity, such as the notion of linear progression, that science and technology are the decisive factors of human development, and that culture can entirely supplant nature. As such, it will appeal to sociologists, social theorists, economists, political scientists, and social philosophers with interests in relational thought, critical realism, and social transformation.

Pierpaolo Donati is Alma Mater Professor (PAM) of Sociology at the University of Bologna, Italy. Former President of the Italian Sociological Association, he is the author of Relational Sociology: A New Paradigm for the Social Sciences, co-author of The Relational Subject, and co-editor of Social Science, Philosophy and Theology in Dialogue. 


\section{Routledge Advances in Sociology}

\section{COVID-19}

Volume I: Global Pandemic, Societal Responses, Ideological Solutions Edited by J. Michael Ryan

\section{COVID-19}

Volume II: Social Consequences and Cultural Adaptations

Edited by J. Michael Ryan

\section{Prevent Strategy}

Helping the Vulnerable Being Drawn Towards Terrorism or Another Layer of State Surveillance?

Edited by David Lowe and Robin Bennett

\section{Regime Change in Turkey}

Neoliberal Authoritarianism, Islamism and Hegemony

Edited by Errol Babacan, Melehat Kutun, Ezgi Pınar and Zafer Yllmaz

307 The Politics of Europeanisation

Work and Family Life Reconciliation Policy

Nazli Kazanoglu

308 Internet Dating

Intimacy and Social Change

Chris Beasley and Mary Holmes

309 Transcending Modernity with Relational Thinking Pierpaolo Donati

310 Exploring Welfare Bricolage in Europe's Superdiverse Neighbourhoods Jenny Phillimore, Hannah Bradby, Tilman Brand, Beatriz Padilla and Simon Pemberton

For more information about this series, please visit: https://www.routledge. com/Routledge-Advances-in-Sociology/book-series/SE0511 


\section{Transcending Modernity with Relational Thinking}

Pierpaolo Donati 
First published 2021

by Routledge

2 Park Square, Milton Park, Abingdon, Oxon OX14 4RN

and by Routledge

605 Third Avenue, New York, NY 10158

Routledge is an imprint of the Taylor \& Francis Group, an informa business

(C) 2021 Pierpaolo Donati

The right of Pierpaolo Donati to be identified as author of this work has been asserted by him in accordance with sections 77 and 78 of the Copyright, Designs and Patents Act 1988.

The Open Access version of this book, available at www.taylorfrancis. com, has been made available under a Creative Commons AttributionNon Commercial-No Derivatives 4.0 license.

Trademark notice: Product or corporate names may be trademarks or registered trademarks, and are used only for identification and explanation without intent to infringe.

British Library Cataloguing-in-Publication Data

A catalogue record for this book is available from the British Library

Library of Congress Cataloging-in-Publication Data

Names: Donati, Pierpaolo, 1946-author.

Title: Transcending modernity with relational thinking/Pierpaolo

Donati.

Description: 1 Edition. | New York : Routledge, 2021. |

Series: Routledge advances in sociology | Includes bibliographical references and index.

Identifiers: LCCN 2020051476 (print) | LCCN 2020051477 (ebook) |

ISBN 9780367705121 (hardback) | ISBN 9781003146698 (ebook)

Subjects: LCSH: Social interaction. | Postmodernism-Social aspects. |

Globalization-Social aspects. | Technology and civilization.

Classification: LCC HM1111 .D668 2021 (print) | LCC HM1111

(ebook) | DDC 302-dc23

LC record available at https://lccn.loc.gov/2020051476

LC ebook record available at https://lccn.loc.gov/2020051477

ISBN: 9780367705121 (hbk)

ISBN: 9780367705138 (pbk)

ISBN: 9781003146698 (ebk)

DOI: $10.4324 / 9781003146698$

Typeset in Times New Roman by KnowledgeWorks Global Ltd. 
To those who strive to see the social relations that, like invisible threads, orient human life 


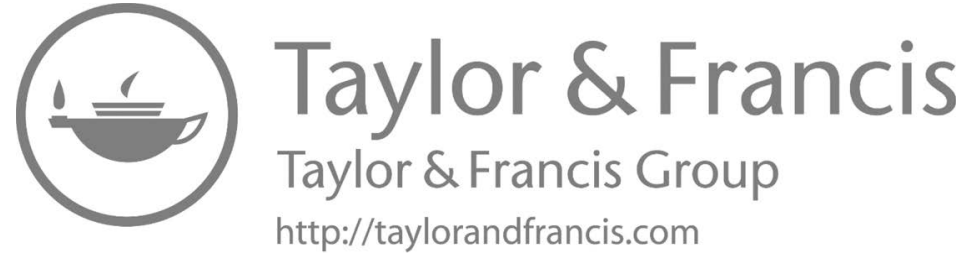




\section{Contents}

List of figures $\quad$ ix

List of tables $\quad \mathrm{x}$

Introduction: Towards a relational society $\quad 1$

\section{PART I}

The emergence of aftermodernity

1 Beyond the modern dilemma freedom (lib) vs control (lab): What after? 15

2 Overcoming the market/state binary code

3 Shedding light on society through a relational (not relationist) gaze 51

\section{PART II}

Insights into the morphogenetic changes of social morality

4 Relational society as a morphogenetic configuration

5 The morphogenesis of social morality

6 Morality and social networks

\section{PART III}

Why and how can the new society be "good"?

7 What does the good life consist of? 
viii Contents

\section{PART IV}

The hybridisation of society: shall we forget about its human character?

9 The new scenario of a hybridised society

10 Prospects: Should we abandon the dream of a human(e) society?

References

Name Index

Subject Index 


\section{List of figures}

1.1 The relational hyperbola of late modernity 30

1.2 The hyperbolic relational nature of aftermodern society at its beginning

2.1 The economic logic of a lib/lab society

2.2 The economic logic of a relational society 39

2.3 Aftermodern society as a societal network intertwining

3.1 Different orders of social relationality: processual (interactional/transactional) and structural 56

4.1 The basic general scheme of the morphostatic/morphogenetic process by which social relations are reproduced or changed $\quad 76$

4.2 The model of the social relation as a "molecule of the social" and its environments

4.3 The structure of the social relation (social molecule) typical of modernity 86

4.4 The transition from modernity to aftermodernity 88

4.5 The molecular structure of the social relation typical of an aftermodern society

4.6 The different meanings of the value added by the relation to something/someone (analytical dimensions of a relational complex)

4.7 The generation of relational goods through social capital (and vice versa)

5.1 How system morality and agential morality are mediated by the morality of social networks

6.1 The interplay between individual and relational ethics

8.1 The morphogenesis of the subject's relational identity (along the time steps T1-T7)

9.1 A scheme of the process of hybridisation (structural, agential, and cultural) 


\section{List of tables}

8.1 Three ways of defining identity 176

9.1 Four types of relations generating hybrids when mixed together

10.1 A comparison between substantialist, relationist, and relational approaches 


\section{Introduction}

\section{Towards a relational society}

\section{Introduction text}

"The real voyage of discovery consists not in seeking new landscapes but in having new eyes" (Marcel Proust)

\section{Premise}

After many years of theoretical work and empirical research on social relations, and on the paradigm of relational sociology, I think the time has come to outline the vision of society to which these reflections and field research lead.

As far as I can see, from the 1980s, from year to year, the relational perspective on society has proven to be increasingly topical. The reasons for this growing relevance are many. I could mention the processes of globalisation, the advent of new technologies (information and communication technologies [ICT], artificial intelligence [AI], robotics), the dematerialisation of society, the diffusion of scientific theories based on the principles of relativity, indeterminacy, the progress in quantum physics, genomics, and many other sciences, not to mention the Covid-19 pandemic which showed the importance of social relations in spreading the virus as well as in avoiding it. Overall, all these factors have accelerated the process of creating a new "relational environment." The various economic crises of the first decades of the 21st century and then the Covid-19 pandemic of 2020 did the rest, making the social relationship the protagonist of social and cultural change, for better or for worse.

This book is an attempt to show how the reality of social relations is profoundly changing modern society and will lead it to be another society, no longer modern as we have known it in the past. Of course, the book is not against modernity, which has brought so many benefits to humanity. Nor is it a question of taking sides for a conservative or progressive position. It is simply a matter of understanding that we are going beyond modernity. It is the exit from one world to go to another world. This exit is as problematic as any creation of a new societal formation. 


\section{Introduction}

\section{What is modernity?}

Modern society has been defined in a thousand ways. Sociology has been particularly rich in contributions. Obviously, it is impossible to mention even a few of the main texts. I limit myself in recalling some representations with respect to which I intend to highlight the novelty of the idea of a relational society. I think about the Capitalist Society (Karl Marx 1848), The Acquisitive Society (Richard Tawney 1923), The Achieving Society (David McClelland 1961), The Active Society - "one that is master of itself" (Amitai Etzioni 1968), the Information Society (James Beniger 1986), and, more recently, Risk Society (Ulrich Beck 1992), Reflexive modernisation (Ulrich Beck, Anthony Giddens, and Scott Lash 1994), The Rise of the Network Society (Manuel Castells 1996), and up to the Knowledge Society that many have talked about.

I mentioned these authors only to point out that the various definitions of society have marked an evolution of modernity characterised by a crescendo of dematerialisation of the concept of modern society.

All the connotations of what has been and still is modern society are obviously linked together. They have tried to capture the various phases and changes of the modernisation processes up to the so-called late modern, postmodern, hyper-modern, and so on. It is not the task of this book to directly address these complex issues, which are rather kept in the background.

The purpose of this book is to argue that modern society encounters a social and cultural morphogenesis that leads to "beyond modernity." We are entering an aftermodern, or trans-modern society that I call "relational society." I propose to speak of a society that abandons modernity and transcends it, while maintaining certain aspects of it.

This new society radically alters the guiding ideas of Western modernity, in particular the idea that society, albeit with ups and downs, is marked by a linear or multilinear progress, that time is quantitative, that science and technology are the basic factors of development, and that culture can entirely replace nature. All these ideas encounter a profound crisis. It must be recognised that the changes are moving in uncertain directions and that the configuration of society opens up to the world of the "otherwise possible." It is, therefore, also possible for society to regress to a permanently worse configuration than in the past. Among the phenomena that contrast modernity, we must mention the fact that time becomes qualitative and circular, that science and technology prove to be the causes of perverse effects, that nature rebels against the domination of culture.

The discontinuity is not just about one or more dichotomous differences between the past and the new that emerges, because it is the same dichotomous thought used by modernity that is put into question. New ground of social phenomena feeds a new thinking about the possible relationships between opposing entities, such as conservation/change, past/future, quantitative/qualitative, and nature/culture. What relationships? 
Having to choose, for simplicity reasons, a dichotomy that represents the most significant nucleus of modernity, as it marks its apogee and its decline, I assume that modernity, as a cultural and structural configuration, is defined by the opposition between two symbolic codes, which I call lib and $l a b$. They are still at work, as evidenced by the race to the Covid-19 vaccine which saw the free market advocates opposed to those who rightly wanted the vaccine to be considered a common public good.

At the beginning, and for a long time, the two codes took on the ideological meaning of liberalism (lib) and socialism (lab), freedom vs equality. The third pole of the French Revolution, fraternité, never had a significant role. Subsequently, the two codes were reformulated in various ways and became increasingly abstract. $L i b$ has become the point of reference for individualistic approaches. $L a b$ has become the point of reference for system or holistic approaches.

The relevant fact, from my point of view, is that $l i b$ and $l a b$ have been and still are used as binary codes $(0 / 1)$ which exclude the "Third." The Third can be interpreted in many ways, but what matters to me is that it is not a combination of $l i b$ and $l a b$ (as in the "Third Way" of Giddens). The Third is what transcends both the lib and the lab. It is another symbolic code. In this text, I will try to explain what it is.

\section{From Georg Simmel to attempts to portray the future of society}

The modern world is characterised by the confrontation between progressives and conservatives, left and right, and lab and lib. This world is over. Neither is the winner, although liberalism seems to prevail at times. Why can we no longer describe society with modern categories? Simply because, in the meantime, society has gone somewhere else. Where has it gone?

Who anticipated the new world, beyond the very important contributions of other classic authors such as Marx, Durkheim, Pareto, Weber, was Georg Simmel. An author who experienced the drama of the "tragedy of modern culture." Simmel argues that the tragedy of culture comes about when the objective culture comes to dominate the subjective culture of the individual. In other words, the tragedy of culture occur when the individual's will and self-development become submissive to the product of its own creativity, without the hope of being able to "reverse" the situation (as Marx prophesied).

As Papilloud (2018: 212) reminds us, to respond to the dissolution of values and social life, Simmel develops all his ideas on the rock of the concept of relation. The relation summarises the interpenetration of three processes: Wechselwirkung (reciprocity effect), Vergesellschaftung (socialisation), and Tausch (exchange). Simmel places different importance on each of these concepts throughout the development of his works. In his late writings, he 


\section{Introduction}

extends the meaning of Wechselwirkung to a cosmological principle of life energy. Papilloud (2018: 212-213, italics mine) writes,

Each of these three concepts describes the same shift in modernity from substance to function, that is, from a social differentiation resting on socially defined positions to a social differentiation that itself defines social positions. For Simmel, this cultural turn in modern society leads to an abstract society in the sense that social forms play an increasingly important role for the definition of these social positions, and finally, for the determination of the possibilities that will make society concrete. To put it in a less abstract way, modern society is one in which social actors, groups, and institutions grow by concentrating ever more content in forms capable of occurring in every possible relation. In this regard, money as well as love are good examples of such modern social forms which tend to be life principles, highlighting the indifference of these social forms towards their contents, and ultimately the exchanges that they mediate, as well as towards the actors, groups, and institutions using them to build society. For Simmel, the indifference of such generalized forms potentially fosters strong social inequalities not only because they tend to be monopolized by the actors but also - and maybe above all - because they reduce the concrete work on relations to an abstract game with social forms. The tragedy of modern culture and modern society eventually finds its sociological expression in this ambivalent situation, where modern society is possible as a concentration of its most contradictory tendencies - which means, at the level of Simmel's relational sociology, that modern society is based on relations which, at the same time, it tends to substitute against social forms.

I intend to resume Simmel's work from where he left it as an exploratory and unfinished project. For him, modernity envelops itself in itself without being able to get out of it. As Beck will then say, modernisation becomes "a systematic way of dealing with hazards and insecurities induced and introduced by modernisation itself" (Beck 1992: 21).

Simmel fragments have been developed in various directions. Luhmann (1998), for example, developed the theme of modernity in terms of shifting from substance to function. The theme of the abstractness of social forms was taken up by network analysis. Relationists, such as Powell and Dépelteau (2013), have constructed a concept of relational sociology understood as an abstract game with social forms.

For me, the key to getting out of modern tragedy would be a new vision of relationality. Simmel saw such a possibility through the concept of Wechselwirkung, but the point is that this concept can only be elaborated with a theory of emergence in morphogenetic processes and with a stratified ontology that he did not possess. 


\section{My proposal}

My proposal starts from the need to develop the Simmelian discourse on the relationship as that reality which constitutes the only solid element in critical conditions of uncertainty, darkness, disorientation, and sense of helplessness. It is a perspective that opposes an abstract, purely relativistic, and procedural sociology.

I start from the assumption that society is not made up of people who exchange something with each other (as in the Aristotelian tradition), but that it consists essentially of social relations acted out by people who belong to a relational social context in which they are embedded. Society is not a space that contains relationships, but it is relationship itself (society does not "have relationships" but "is relationship").

What I mean by social relationship is said in all the chapters of the book, in particular in Chapters 3 and 4. Along the whole text, the reader should keep in mind the polysemic and ambivalent semantics of the concept of social relation, which has a double meaning as a process and as an outcome of that process. It may be helpful to consider the analogy with the concept of "association" which denotes both the process of becoming fellows/members (i.e. joining) and the outcome of this process, that is the association as an organised entity with its structure. In the same way, when we say that two agents/ actors "are in relation" or "have a relation," we can mean the process of reciprocal action (interaction and transaction) taking place between them or the product of this process, that is the emergent effect, which is the social relation as a new reality existing between them with its own structure. The latter is a social structure to the extent that it operates as a stable connection between the positions of those who are in relation. Therefore, from the ontological point of view, the social relation can be viewed both as an element essential to the process of emergence and as an emergent (structural entity) in itself.

What I care to anticipate and underline here is my critique of sociological relationism based on a radical pragmatism grafted on that postmodern culture that enhances relativism, processuality, and indeterminacy, a perspective to which Simmel himself tried to react with his Lebensphilosophie.

All relational sociologists agree that we are facing a "relational turn," whose common denominator is the rejection of both methodological individualism and holism. But they give very different interpretations, generally of two types, sometimes combined with each other (Luhmann's theory is an example). These two types are objectifying relational sociologies (e.g. structuralists and systems theorists) and subjectivising relational sociologies (e.g. action theorists and those who claim that knowledge is totally observer-dependent). Neither of them satisfies me, because they don't have a properly relational concept of social relationship as I conceive it.

These two ways of looking at social relations are in continuity with the foundational symbolic code of modernity, which I call lib/lab, where lib stands for subjectivity (free agency) and lab stands for constraint or bond 


\section{Introduction}

(social structure). The two polarities are continuously transformed and recombined while keeping one point still: the exclusion of the Third. The Third is precisely the relationship.

In the past decades, there has been a wide debate on the agency vs structure issue, which has tried to connect the two polarities. But, in the end, most scholars placed themselves either on the side of the agency and the transactions it triggers (e.g. Emirbayer 1997 and his followers, who devalue the structure) or on the side of the structure (e.g. Luhmann 1995 and his followers, who consider the agency as an environment outside the system).

The search for a micro/macro link has not paid off, simply because these ways of thinking do not see the relationship between agency and structure, and, for this reason, they do not include the meso level in which the Third is located.

I consider the morphogenesis/morphostasis scheme elaborated by Archer (1995) as a fundamental methodological tool to highlight what connects micro and macro, that is relationships at the meso level, where the linearity of time leaves room for its circularity, and quantitative time becomes qualitative. I must add that, differently from Archer (2003), I think that the link of conjunction between agency and structure is not personal reflexivity, but relational reflexivity, which entails the fact that the relationship has its own reflexivity (the relational feedback mechanisms).

In this text, I will try to explain why and how the relational turn leads to a deep discontinuity with the qualifying key concepts of modern society. By way of example, I list some of them.

1 The Marxian concept of capital, precisely as a social relation (Shaikh 1990), has been radically changed: on the one hand, at least in advanced countries, it is more and more limited and regulated against the exploitation it entails, reoriented for social purposes and re-embedded through corporate social responsibility, the civil economy, and so on; on the other hand, it is redefined as social capital, which consists of relationships of trust, cooperation, and reciprocity.

2 Durkheim's concept of division of social labour, from functional and organic, becomes relational, that is it becomes networking; the functional differentiation that, according to Luhmann (1995) characterises modernity through binary processes that enhance functional specialisation, gives way to forms of relational differentiation, which follow non-functionalist or suprafunctional logics.

3 The Weberian concept of bureaucratisation of society is radically modified by what I call the Digital Matrix surveillance system (algocracy), which destructs hierarchies and favours a more horizontal, reticular society.

4 The condition of incredulity towards metanarratives, which according to Lyotard (1984) characterises postmodernity, erodes the metanarratives of the past, while new metanarratives are born, such as 
those of the post-human and trans-human, the new ecological cultures oriented to a reappropriation of nature, and those of new religious movements.

Clearly, modern society does not disappear in an instant, it continues to exist. What I want to underline is the fact that, through these transformations, a "relational society" is born that generates unprecedented social forms that escape the old oppositions, such as conservation vs progress, freedom vs control, agency vs structure. On the one hand, the new forms seem to require a liquid and fluid society, but on the other hand, they seek a new connective social fabric.

From an ontological and epistemological point of view, the turning point lies in taking note that human persons are neither the architects of social relations, nor actors who passively perceive them. Interhuman relationships need to be redistinguished from other types of relationships. System reflexivity is by no means sufficient. Personal and relational reflexivity are needed.

My thesis is that only a realist and critical relational sociology accounts for the fact that human beings are both internal and external to social relations, and therefore to society, while constructivist-type relational sociologies consider human agents/actors it either internal or, vice versa, external to society. The relational society of which I speak can be envisioned only if humans are seen as operators of the boundaries between the inside and outside of society and are capable of transcending what exists in a given situation. Human subjects are positioned on the border between the inside and the outside of the structures that condition them, and by acting on the borders, they can change them.

To see the borders properly, one needs to get out of the binary code inclusion/exclusion, according to which one is on one side or the other, totally included or totally excluded, without any mediation. I argue that there is another way of seeing things. There is what I call "relational inclusion," which allows human beings to look at society from the outside, even though they are included in it, and vice versa. This can be seen only with the "relational gaze."

If relationality (not communication, or culture, or structure, or agency per se) is the constitutive fact of society, then agents (individual and collective) are certainly embedded in a certain context, but potentially also capable of transcending their social relations and positions. The ability to transcend given society derives not so much from scientific discoveries and technological artefacts but from the ability of human beings to change the boundaries between their interior (internal conversation) and their exterior (the relational context), because they can only live in the relationship with others, so that existing social forms can be transcended only by transforming these relationships, with and through them.

The Covid-19 pandemic forced us to "measure" relationships, their properties and qualities. The next pandemics will do the same. All epidemics have the task of showing us that the social relationship is not only a vehicle, 


\section{Introduction}

a channel through which the virus passes, but it is also the remedy if handled properly. In a sense, we can say that the relationship brings the virus, but it is also the means to avoid it. Relationships decide the quality of our life and our destiny. They do it for better or for worse. We need to measure the distance and strength of the relationship, its qualities and causal properties. Getting sick during an epidemic like Covid-19 has strong analogies with being prey to other kinds of pathogens, such as fake news carried by ICTs or harmful consumption offered by the market. Social relationships must be measured in terms of penetration capacity. Knowing how to involve and detach ourselves from others becomes fundamental.

The underlying imperative of this book is the need to empower our "culture of relationships." Relationships are ambivalent and enigmatic because they can generate good or evil, and therefore require competent observation and evaluation. They are a reality different from our feelings or ideas. Normally, we do not see them, because they are immaterial and invisible like air. Precisely for this reason, they have the power to manage us if we do not know how to map and handle them. However, relationships are never what we want them to be. They are never just the projections of our interests. They affect our lives even if we are not aware of it. Hence, there is a challenge.

\section{Contents of the book}

The target of this book is to show the power of social relations in generating a new society beyond modernity. To understand this epochal change, the book proposes to equip us with a new ontological and epistemological paradigm that I call "relational thinking."

Part 1 is intended to explain the exit from modernity.

The thesis of Chapter 1 is that the transition from modern to aftermodern society is driven by the need to overcome the dilemma freedom/control distinction to go towards a post-lib and post-lab society. We must seek a sociological approach which may allow us to see the new, historically unique aspects of social formations that are taking place before us more clearly.

Chapter 2 deals with the recurrent societal crises connected to the processes of globalisation. We are still short of a deep sociological interpretation of these crises differing from interpretations centred upon economic, moral, and political factors. My argument is that, in order to avoid permanent societal crises, we have to break away from the self-referential logic of the lib/ lab configuration of society. Such a new set-up shall be capable of allowing for the emergence of new social subjects that can generate and adopt certain ethical standards of conduct and change the economic-political systems. This transformation should be structural. Luhmann would say that this is not possible, because - in his view - society operates with autopoietic mechanisms insensitive to social subjectivities. I propose to meet the challenge of Luhmannian sociology by showing why and how the emergence of a new society is as possible as it is necessary. 
Chapter 3 tackles this challenge by suggesting that the solution lies in having a "relational gaze" on social reality. Today, many sociologists invite us to see social "objects" (societies, institutions, social movements, social classes, etc.) in a processual way, that is as being made of always fluid, dynamic relations. In my view, they offer a reductive and misleading vision of social reality, because they rely upon a flat social ontology rather than a stratified social ontology, so that social relations are reduced to eternal flows underestimating the weight of structures. On the contrary, in my view, a truly relational sociology should attribute a relative autonomy to structures, even if they are produced by processes. The relational gaze distinguishes between different orders of reality, the processual-interactional and the structural one, so to be able to see how ethically oriented interactions can generate new economic and political structures. It can show how the economic, the political, and the ethical are intertwined with each other. The topic is expanded upon in subsequent chapters.

Part 2 is intended to clarify how a new society is generated through morphogenetic processes, in which the moral dimension of social life plays a fundamental role.

The aim of Chapter 4 is to understand how the morphogenesis of society comes about through social relations, which are the connectors that mediate between agency and social structure. The generative mechanism that feeds social morphogenesis resides in the ways in which social networks alter the social molecule of the structures already in place. From viewpoint of relational sociology, social morphogenesis is a form of surplus of society with respect to itself. This surplus is produced through the relationality that agents/actors create in their interactions, so as to alter the initial structures. The morphogenetic surplus is not so much the product of structural effects as of "emergent relational effects." Society increases (or decreases) its potential for surplus depending on processes of valorisation (or devalorisation) of social relations. Empirical references are given in order to show how the lib/ lab societal arrangement is overcome by an aftermodern society that can be called "relational."

Chapter 5 explores the germs from which a relational morality could arise. In modern social sciences, moral norms are generally explained as a product of individual actions or as a manifestation of adaptation to sociocultural institutions. In recent decades, theories that combine these two paradigms in various mixes have multiplied in so-called institutionalised individualism, a distinctive trait of Western morality. In my views, these modalities for explaining morality reflect the structural and cultural dualisms of modern Western society (based on the individual/State, private/ public, and micro/macro axis) and their compromises, which today face a profound crisis. The basic thesis is that moral norms are meeting processes of change characterised by the need for new relational behaviours between humans and between them and nature. The emergence of a trans-modern social morality takes place in those social spheres in which social relations 
are considered a reality endowed with sui generis qualities and causal properties, so that relational normativity becomes the fundamental moral criterion of new social practices.

Chapter 6 focuses on morality of social networks. Premodern traditional ethics has imputed the morality of action to the "acting subject" on the basis of a principle of linear causality. With the advent of modern social sciences, the social, economic, and cultural conditioning weighing on the human subject have been evidenced. The result has been to attribute moral responsibility to a "conditioned subject" who, in turn, attributes the responsibility to the existing societal structures, including so-called unjust laws. In this way, responsibility has become anonymous, and its weight has been transferred to the welfare state. A sort of vicious circle was generated, which now can only be broken by new relational subjects, both individual and collective, who take responsibility for social networks that produce relational evils at a distance. Ethics is required to make itself relational, in the sense that the attribution of responsibility for acting for good or bad cannot be limited either to a single act or to structures, but must invoke the reflexivity of relational subjects and the reflectivity of social processes that take place in networks of relations. The ethics of intention is no longer sufficient. It must be integrated with an ethics of responsibility that is not restricted to the direct consequences of individual acts but takes into account the indirect consequences of relational networks.

Part 3 tries to identify who are the bearers of a societal change that can avoid the relational evils to which the lib/lab configuration leads.

In continuity with the previous part, Chapter 7 wonders, in which ways and why do hyper-modernised societies change the concept and practices of the "good life?" What are the prospects for the next future? Human happiness is being redefined as the possibility of enjoying opportunities that present themselves in contingent situations. It becomes a matter of analysing who offers these opportunities, how they are used, and what effects they produce. I argue that there are three main sources offering opportunities: the lib/lab systems, a global communication matrix of an impersonal nature, and new collective subjects of civil society organised in social networks. The opportunities afforded by these three sources are selected on the basis of a multiplicity of logics (individualistic, systemic, or relational). I claim that these different moralities of the good life are generated according to different ways of addressing the relation between "the social" and "the human," and, more generally, according to the diverse ways of considering social relations as the decisive reality fostering human fulfilment. In the end, my thesis is that, in a society conceived as a field of opportunities, the discriminating factor of "living well" becomes the relational or non-relational nature of the good that is sought and realised by the acting subjects. This guiding idea is what supports relational social work in dealing with welfare and well-being issues. The good life is a matter of the modalities with which 
agents and social networks produce relational goods or, vice versa, engender relational evils.

Chapter 8 tries to clarify in what sense and in what way relational subjects are emerging. After the publication of The Relational Subject (Donati and Archer 2015), the issue of how to connect personal reflexivity (i.e. inner conversation) and social reflexivity (i.e. how social relations affect the identity of the subject reflexively) has remained opened. In this chapter, I propose an answer to this problem from the point of view of relational sociology. The argument is that (i) it is possible and necessary to distinguish between two kinds of reflexivity (inner and relational) and their different contributions; and at the same time, (ii) we need to clarify their complex connections in the process of structural and cultural morphogenesis of the identity of the agent/actor. A conceptual framework, capable of empirical verification, is proposed that can explain the relational constitution of agents/actors beyond the limits and fallacies of both individualist and radically relationist approaches.

Part 4 deals with the challenges to the relational society that come from the hybridisation of social identities and relations with new digital technologies.

Chapter 9 examines the phenomenon of hybridisation of social relations. The enhancement of human beings through digital technologies compels us to evaluate whether, how, and when these technologies feed the flourishing or, vice versa, the alienation of humanity. Social identities, relations, and organisations are forced to take shape in the environment of a Digital Matrix that is a symbolic code which tends to replace all the ontological, ideal, and moral symbolic matrices that have structured societies in the past. In a certain sense, the Digital Matrix I am talking about is a functional substitute for that metaphysical conception that Leibniz, in his Dialogus (1677), outlines by saying that "the world is formed thanks to the thought and calculation of God" (cum Deus calculat et cogitationem exercet fit mun$d u s)$. In this new vision, God's place is taken by the symbolic code of the Digital Matrix. The great calculator is no longer the divine person, and certainly, not the human person, but an anonymous system that circulates and pervades the world.

As a form of TechGnosis, the peculiarity of the Digital Matrix is that of making the boundaries between human and non-human labile and crossable in every way in order to foster hybrids. Hybrids, however, are not random or purely contingent entities. They stem from complex interactional networks, in which social relations are mediated by the Digital Matrix. The processes of hybridisation of social identities and relations are selective and stratified according to the ways in which the human/non-human distinction is thought of and practised. Three scenarios of hybridisation are outlined along with three kinds of societal morphogenesis: adaptive, turbulent, and relationally steered. The chapter examines these alternatives and their possible consequences. The Covid-19 pandemic of 2020 had the effect of 


\section{Introduction}

accelerating the digitisation of all societies. The relational society will therefore be increasingly virtual.

Finally, in Chapter 10, I argue that the great challenge is to think whether what we call "society" could retain its basic human characters or whether it is inevitable that it will become post- or trans-human. The thesis is that the recognition of the human character of social life depends on whether the human is essentially distinguished by its own species-specific relationality. It is a question of rethinking human identity by passing from unrelated thinking, which reifies or subjectivises identity, to relational thinking, for which the essence of human identity lies in the fact of being a sui generis relational subject. It is the relationship with the Other (not the face of the Other as such) that tells me what I actually am. The human character of society is either relational or it is not. The human feeds on a relational rationality, far beyond instrumental and value rationality, otherwise it does not surpass itself but, on the contrary, it regresses or degrades in the non-human.

What we call "relation" belongs to all the orders of reality, it is a way of existence of all existing entities (existence takes priority over essence). Everywhere, but particularly in the case of human beings, it presents itself as an enigma. In the end, what I call "relational society" may not be the social configuration that people dream of. However, if we increase our awareness of what it is, we can perhaps limit the tragic conflicts, wars, and exploitation of human beings that bloody the world, or even simply we can avoid indifference towards our fellow human beings and the creation. It can be an antidote to a society dominated by technocracy and algocracy. Technology is capable of destroying humanity. But, as long as there are human beings who cultivate their humanity, technology will not be able to dominate the world, because it will have to go through the Caudine forks of meaningful social relations. 


\section{Part I}

\section{The emergence of aftermodernity}




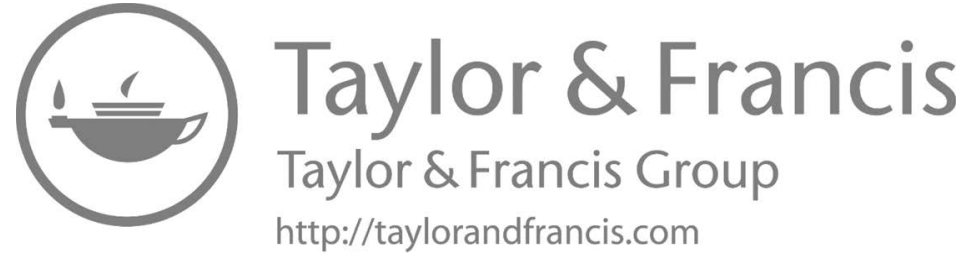




\title{
1 Beyond the modern dilemma freedom (lib) vs control (lab)
}

\author{
What after?
}

\section{Modernity as a lib/lab dilemma}

Modernity contains a fundamental dilemma. It was born based on a specific antithesis between freedom and social order (the latter in the sense of control), as polarities irreducible to one another within which social life unfolds. Freedom is generally thought of as the possibility of action unbound by conditioning. On the contrary, order (control) is intended as a bind that conditions action from the outside. Conditioning and binds are first conceived as naturalistic, then as normative, and finally mechanical.

The point I want to underline is that, to moderns (both liberal and non-liberal), freedom lies outside control (i.e. extrinsic to its forms). Social control as such cannot make one free, cannot be a component of freedom, but only expand or diminish the chance for freedom, which is built on other foundations. Freedom lies in the subject (individual, collective, or historical), while social control lies in external constrictions (in the form of rules, structural, normative, or functional).

The previous statement may be mitigated by saying that both liberty and control are conceived within a shared framework characterised by rationality, contractuality, and conventionalism. However, dualism remains.

Some have observed that Western social thought, compared to other cultures, places freedom ("liberation" of the subject, beginning with the individual) as a priority and as a limitation of control. Of course, only in the West do we find radically libertarian theories, unknown to other societies. But it would be stretching to maintain that modern social thought interprets society only as a process of liberation or, vice versa, only as a process of control, although these unilateral temptations are anything but absent.

We can instead state that modern social theories still differ today on the side of the distinction (freedom vs control) from which they choose to observe society: some see society from the side of freedom and as a function of freedom (I will call these lib theories), while others see it from the side of control and as a function of social control (I will call these lab theories). Either it is $l i b$ or $l a b$, the third is excluded. The fundamental binary distinction is that of freedom/equality. Some might object that these are 
not antithetical terms, since "equality of the conditions of freedom" also exists. But one might respond to this objection that the conceptual category "equality of the conditions of freedom" is paradoxical, and therefore does not eliminate the binary nature of the lib/lab distinction. Indeed, sociological analysis reveals that the processes that encourage liberty are against social equality, and vice versa social controls are introduced to reduce the inequality deriving from the existence of certain freedoms.

As modernity develops, the "lib/lab complex" increases, in which the two poles - lib and lab-combine and are gradually placed in increasing synergy. Distinctions are no longer seen as objective differences but as social constructions. Modern social thought legitimates a configuration of society in which $l i b$ and $l a b$ feed one another, however opposite they appear. Through this process, classical liberal thought finds its end. This is the framework that should be highlighted.

Observing social reality from a lib/lab standpoint has certain consequences: (i) it leads to theoretical paradoxes and (ii) it contradicts many aspects of empirical reality. Modern and contemporary social theories raise these two sets of problems. In an attempt to address these problems, modern social thought transforms its very nature: from an explanatory and/or interpretive narration of social reality, seen as a phenomenon that emerges spontaneously, it becomes a means for the paradoxical construction of social reality itself.

Those who have sought a non-paradoxical composition between freedom and control within the paradigms of modernity, specifically Talcott Parsons, have failed. No matter how hard they try, social theories which refer to the classics (up to and including Parsons) do not see how freedom and control can be reconciled, in the sense of mutual support or at least significant interdependence with one another. Freedom and control are assumed as two tracks - infinitely parallel - along which social theory runs, but nothing is said about how they are intrinsically connected. Social thought therefore finds itself with the continuous need to return to the discussion on the meaning of the categories of freedom and control per se. In doing so, it generates theories that are by necessity anti-modern, neo-modern, or postmodern.

This highlights the fact that modernity has made a bet. It has configured the relationship between freedom and control as a typical synergic antithesis (i.e. an antithesis producing a synergic effect) between the two terms of the distinction. But today this bet seems about to be lost. Indeed, in today's Western society, we can note that the contingencies for both freedom and control are increasing, that both sides tend to pursue their own paths independently, that their meaningful bonds no longer hold - at least those that were considered meaningful until recently. This very fact marks the end of classical liberalism (Gray 1993). This highlights the fact that, today, the union between $l i b$ and $l a b$ has become an "unhappy marriage."

The relationship between liberty and control cycles endlessly and ends in a void, or it remains limited by forms of self-understanding (the lib/lab 
complex) that prevent society from developing new meaningful and more stable relations. As a matter of fact, if the lib/lab logic is radically extended to all forms of social life, it generates catastrophes. If instead it is restricted to inhibit further possibilities for synergy, it runs the risk of leading to degenerative processes, for example a regression to pre-modern forms of social life or a leap into postmodern destructuration. Modern management of the freedom/control coupling becomes increasingly problematic.

At the beginning of the 21 st century, many governments attempted to reintroduce a neoliberal reading of society understood as a system that can simultaneously increase freedom and social control, making both more rational, contractual, and conventional. But this is an illusory dream. The synergy no longer acts as a guiding criterion for all of society. The freedom/control distinction is reduced to a mere conceptual pair, analytical in nature, that no longer grasps the meaning nor the functions it held in modernity. One wonders, then, whether modern social theories that reason in terms of lib/lab might contain some actual ideological biases based on a type of society which now appears obsolete.

Theories that remain within the lib/lab framework see subjects and rules, but not the generation of society. Generating society becomes - peculiarly, for the first time in human history - building a network of communicative relationship networks.

The thesis of this chapter is that the passage from modern to aftermodern society is specifically distinguished by the need to move towards a relational approach to the freedom/control distinction, which is post-lib and post-lab, post-individualistic, post-holistic. Such an approach may allow us to see the new, historically unique aspects of social formations that are taking place before us more clearly.

\section{Lib and $l a b$ meet and combine}

In modern social thought, despite the debates between lib and lab thinkers or, if we prefer, methodological individualism and holism - society is seen through the common framework of a historical process that conceives itself as individual and/or collective liberation from the ascriptive ties of the community (Gemeinschaft) to move towards progress in which Reason, be it individual or collective, micro or macro, of action or social systems, leaves its contract mark on society.

Within this scenario, freedom is intended as freedom "from" (thus as an opening of contingencies of existence, and not merely dependencies), rather than as freedom "for" something or someone. And within this framework, social control is intended as external coercive regulations rather than an intentional and purposeful choice according to a moral conscience inherent in the subjects and their relationships.

Some might object that this is simply the positivistic, functionalistic side of social thought, so to speak. I feel the same way. But the point is 
that within modernity, no great Western social theory appears immune from creeping positivism, which even pervades those theories intended to be non-functionalistic or even anti-functionalist (Marxian theories for example).

Why is positivistic functionalism considered to be limiting and simplistic, but then permeates every theory and ends up winning in the end?

I believe the reason lies in the fact that $l i b$ and $l a b$ theories are not truly opposites, but largely complementary: they "dance together," so to speak. This very dance is what feeds positivistic functionalism. Freedom and regulation, whether aimed at the individual or collective, work together in a certain way (to be defined later) to build that symbolic and institutional complex (lib/lab) that contains the collective consciousness of what modernisation means and what are deemed to be "good systems governance" under normal condition. Different is the case in which there is a need to face serious economic crises, pandemics, and catastrophes, as we will see later.

The categorical imperative says: we must expand all freedoms under the sole condition that they do not create a constriction for anyone; and good governance is considered an expansion of all possible freedoms as long as they are "compatible" with one another and with the concurrent principles (especially equality) that act as external binds.

From a methodological standpoint, this means that individualism and holism "shake hands," support, and complement one another. Society is conceived as a game between freedom and control along the individual-government axis, through continuous renegotiation between the market and the state.

Even empirical research sees through the same lenses. To give an example, anyone wishing to prove that school choices are individual rather than induced by the societal system can do so. However, in the end, one must take note, on the basis of empirical research, that the growth of individual freedoms in choosing school careers does not alter the stratification structures of the whole society (Boudon 1981). Individualism and holism meet and shake hands.

This is the lib/lab paradigm inherent in modernity. It proposes a synergy between freedom and social control that constitutes the "propulsive engine" for the entire historical-social formation. The engine works like this: social control is used to free individuals, and freedom is used to make control more rational and functional to progress, under the assumption that one can be freed from the binding nature of social relationships without putting social order in deep danger.

Even after the economic crises, global warming, pandemics, or other catastrophes, Western social theory thinks of society in these terms: as the battle between the forces of freedom, representing the propulsive (innovative) thrust, generally free of any need for a priori ethical justification, and the forces of social control, representing a brake (self-preservation safety) and generally requiring justification, which must become increasingly technical-functional. The burden of proof is on control. The brake refers to the public sphere and must be used only when others' private freedom 
is violated, not before and not for any other reason. The fuel for the history machine is the liberty/control distinction used as a synergic antithesis between the private and public. Synergy means the use of the public to privatise the private (legislation must increase private freedoms) and, vice versa, the use of the private to publicise the public (the more freedoms grow, the more public control must be increased to guarantee them). The development of one is based on the development of the other, even if "at a distance," because controls are entrusted to the system, while freedoms float in the environment of the social system.

The engine of the modernity machine, thus configured, is fuelled by a potentially infinite energy - or at least that is how it is represented. Progressively removing constrictions to freedom, making it potentially unlimited, means creating an inexhaustible source of resources. If one then manages, in a complementary fashion, to invent a form of social control that does not block this process of liberation, but instead uses control to expand freedoms, for instance using new technologies, then social control itself is no longer an insurmountable obstacle, but rather a mere identification of temporary limits and functionally necessary mechanisms (including automation) to ensure that freedoms run smoothly.

Those forms of society that interrupt this process are viewed as deviations, pure accidents, temporary halts, or stopovers. This is how we interpret, on the one hand, political dictatorships (whether they be communist, fascist, Nazi, or other types) that eliminate civil freedoms and, on the other hand, those forms of capitalism considered rampant or haphazard (casino capitalism), which do not guarantee equal freedoms for all. In the eyes of the lib/lab paradigm, dictatorships and unregulated capitalism are "unintentional effects," which must be once again subjected to the (same) freedom/control directive distinction. Modernity is convinced that the lib/lab machine is expandable in terms of progressive upgrades. It refuses the idea that this logic may generate situations that are more problematic than before.

The dance where $l i b$ and $l a b$ shake hands is still the prevailing arrangement in many countries. In the meantime, however, its limits have become apparent. We are gradually realising that it prevents the observer from seeing beyond the horizon where other possibilities have their place. Indeed, many possibilities are not at all thematised or discussed, and many of those that are considered probable in the end prove more virtual than real. In brief, today one realises that the lib/lab approach does not see the morphogenesis of the dialectic between freedom and control that escape modern logic.

\section{The modern freedom vs control dialectic leads to paradoxes and is challenged by empirical reality}

The lib/lab configuration begins to be placed under serious discussion when it encounters systematic malfunctions, thus when one realises that it can no longer function structurally. 
There are many crisis paradigms. Most of these take note that the modern paradigm of development encounters structural limitations in its external and internal environments (to cite just one author, see Hirsch 1995). Within the system, the synergy between giving more freedoms, on the one hand, and organising social controls capable of ensuring these freedoms, on the other hand, produces more problems than solutions. As for the relationship with the physical environment, one realises that we cannot exploit nature (physical resources) indefinitely. On the whole, the increase of pathological repercussions becomes evident.

Yet, not everyone sees that critical results are the product of specific relationship processes between freedom and social control that generate vicious or perverse circles. What I would like to emphasise here is that the lib/lab complex in itself leads to situations that are unsolvable paradoxes and that contrast mightily with the needs and experiences of daily life. Let us examine these two aspects.

1 In the first place, to describe society specifically as a synergic antithesis between freedom and control leads to unsolvable paradoxes because: (i) freedom enters into contradiction with itself and (ii) social control loses its legitimacy. Let me examine these separately.

a The exaltation of freedom as the absence of normative restrictions internal to the agency and its subject spreads social anomie and ultimately causes the self-destruction of the same freedom. In the symbolic code that modernity generates, it is written that freedom as interdependency is a purely temporary limitation, because freedom as such consists of the possibility of abandoning interdependency. The growing monetisation of social transactions, in which money is transferred as long as the possibility of avoiding any constraints in future transactions is increased, is the prototype of this evolution. The value-pattern of society becomes the convertibility of anything into anything else.

As long as this process remains restricted to limited groups of people (the modern élites), only the highest social classes experience a lifestyle in which freedom is an end unto itself. Only they, for the moment, enter the paradoxes. When the process becomes a mass phenomenon, one realises that all of society takes on the same characteristics. We are at the "drift of liberalism," thus within a social arrangement in which freedoms cancel each other out. The very idea of civil comes under discussion (Seligman 1992). To generate a civil life, one must create new social institutions which - far from encouraging a "lightening of ethics" - manage to intertwine freedoms and controls through morally significant relationships between them. When the old relationships fall, one must seek new rules for the creation of social institutions that connect freedom and control in a different way. 
Since it is imperative to be modern, and since we are modern to the extent to which we do not seek rules which can constitute freedom from within, but only mechanisms to reduce the undesired effects of freedom, social norms come to feed vicious circles within the system of freedom. To put it bluntly: many private behaviours are permitted, but their public effects are punished or blamed, as happens when a country permits a certain use of drugs in the private sphere but punishes those who sell it and blames those who become drug-addicted.

b Something similar takes place for control. A concept of social control as external and coercive towards the agency and its subject causes a substantial delegitimisation of the social order, because the controls do not have the necessary consent of the people. Therefore, the mechanisms (institutions and rules) that should guarantee the social order lose their effectiveness.

The more social rules are separated from the subjects' motivations and interior aims, the more they are perceived as purely artificial and arbitrary, and therefore free of any human sense; they become a pure technical necessity, which spreads an adiaphoric ethics.

There are abundant indicators of these results. We might briefly recall: the collapse of the institutional welfare state; the fact that the law has changed from guarantor of social order to a source of social disorder. In all of these instances, social control is first delegitimised in terms of aims and values, and then reduced to a technical point and thus subject to procedural rules that chase their own tail (Dahl 1979). For example, the welfare state was created to achieve greater social justice, but was then reduced to a functional fact of redistributing resources, thus making the ethos of welfare more and more evanescent.

The control machine begins to waver. This phenomenon may be described as the emergence of an order based on increasing entropy. Some, using other languages, speak of the death of public goods, while others declare the death of privacy. But few see that these demises are produced by that very modernity that exalts the lib/lab synergy. The outcome is due to the prevalence of negative freedoms (devoid of moral norms, i.e. intended as freedoms "from" something or someone) over positive freedoms (those that are oriented towards - they are "for" - something or someone). The latter see the Alter not merely as a limit, but as a condition and resource for Ego's agency and its responsibility (responsibility understood not only as a due response, but as taking care of the Other). In this case, Ego is free to act insofar as he/she can promote Alter's freedom as a condition and resource of his/her own action. Contrary to the unrelated character of the concept of negative freedoms, the positive concept emphasises the relational nexus existing between Ego and Alter, which becomes the focus of sociological interest. 
2 In the second place, the lib/lab code contradicts empirical evidence and subjective experiences of daily life. Modern social thought describes the relationship between freedom and control as a synergic antithesis that can find new mutual adjustments for better solutions of social problems. But that is not how things really are.

In common-sense experience, the growth of freedom is always problematic, as is the growth of social controls. To conceive of society as a "society of individuals" (Elias 1978) with increasing individualisation, that is as a society capable of "individualizing individuals" (Beck 1992) by loosening social constraints, appears to be highly misleading for at least two reasons: firstly, because it underestimates the fact that the individual is prey to new powerful collective forces linked to fashion, social media, and consumer market and, secondly, because it does not mention that, as many authors have shown - such as Foucault and Donzelot - the institutionalisation of individualism may transform the "technology of self" into the destruction of the self.

It is beyond a doubt that new freedoms appear on the one side and new controls on the other. Nevertheless, this growth is not parallel. Most of the time it is asymmetrical in space and time. It remains highly problematic in the rules that guide social processes. The idea that social control may be configured in such a way as to ensure greater individual freedoms without significant relations between freedom and control leads to mystifying forms of system control, which bring about the schizophrenia typical of daily life in our times.

The experience of contemporary men and women is that they live between two entirely discrepant levels of reality. On the one hand, they are theoretically free to do anything they like, on the condition that it remains private. The culture of globalisation reinforces this feeling in them, which is that they may "privately" enter the realm of virtual reality, so to speak. On the other hand, when they deal with actual deeds, men and women find that the opportunities to satisfy their needs are socially limited and structured. Many specific freedoms and identities are denied. The ideology of egalitarian control ensures that men and women can have the same freedoms and opportunities, but in practice, the opposite occurs. Social freedoms and opportunities are gendered, that is differentiated by gender, and the egalitarian viewpoint does not allow us to see the new inequalities that are generated. And yet the globalising machine of modernity provides a representation that denies this fact. It can admit the existence of inequality only as a temporary situation, pending the continuation of the antithesis of synergistic lib/lab in an attempt to generate forms of control that ensure greater freedom, which does not happen.

The conclusion is that, in the globalisation processes, the ideology of freedom masks widespread non-freedoms, and the ideology of equality masks new inequalities. 


\section{Attempts to reconcile freedom and control conducted within modernity}

Parsonian theory represents the finish line and point of greatest inertial equilibrium in modern sociological thought between freedom of action and the need for social order, between private and public, or, to use the words of Jeffrey Alexander (1983), between substantial and formal voluntarism. Parsons is the last of the classics to theorise the lib/lab framework as the optimal solution that allows that contact between reason and religious revelation which, according to many (Seligman 1992), is the origin of the modern spirit and its idea of freedom (civil society). To him, this configuration is a structural form that conditions freedoms in order to ensure the achievement of common goals in a liberal democracy. In other words, for Parsons, the lib/lab ensures the maximum possible fit between system integration and social integration, ${ }^{1}$ if not the first-best Pareto optimality.

In Parsons (2007), the societal community may combine freedom and control only because it still retains something that transcends both. It incarnates the spirit of a freedom that is born "from within" social actors, since it rests on a cultural system with religious roots. After Parsons, sociology is no longer able to justify this arrangement, which appears to be grounded in an unduly normative vision of society. Because of how his theory (read: the original AGIL diagram) is formulated, it absorbs and rationalises the transcendent element, the vital source (of values) of the social system. Within the very logic of Parsons' (non-relational) AGIL, the subject of freedom disappears against the determinations and structural limitations of social action. Indeed, in Parsons (1967), it is already evident that civil society cannot stop the secularisation of transcendent (i.e. religious) concepts and values.

With Parsons and immediately thereafter ends the dream of the starry heaven above that is reflected within us. The Kantian spirit of modern social thought dissolves. It is no longer so easy to reconcile freedom and control. The lib/lab synergy can no longer be considered a normally functioning process. Normality becomes the very fact that the mutual conciliation of freedom and control no longer works. Society becomes a "deviant majority."

Parsons thought that freedom consisted of individuals internalising the value-patterns and control mechanisms of the Weberian Protestant ethic, and Freudian schemes in socialisation terms. Both one and the other have been lost. Thus, it is revealed that this kind of thinking was modern only in part; it actually reflected a few pre-modern convictions. In its specific constitution, modernity thinks that freedom cannot be founded on value normative control. This is the exact point where Parsons fails and must be abandoned.

1 For the distinction between social integration and system integration, I refer to Lockwood (1992). 
It becomes clear why and how Parsons never found a way out of the dilemma that lies at the heart of all modern sociological theory, and that can be expressed by the guiding question: how is it possible to limit the (modern) social system's demand to control all areas of human life, beginning with the pretence that something (nature? religion?) comes before the system and that, through it, legitimises its institutions?

Parsons (1977) theory still assumes that (i) freedom and control work within a certain symbolical framework of values and (ii) they increase each other by respecting the famous cybernetic hierarchy of control (latency $\rightarrow$ integration $\rightarrow$ goal attainment $\rightarrow$ adaptation). But both of these conditions have collapsed today. Thus, the guiding question becomes: how can the modern social system develop, or even survive, if it globalises contingencies and cancels the control hierarchy? Parsons' theory becomes useless.

Sociology in recent decades is a declaration of the failure of Parsons' theory as the apex of modern theorisation on the freedom/control dilemma.

Three alternatives arise after Parsons:

a run the risk of being anti-modern bringing freedom back under the aegis of social control;

b support the path of a neo-modernity by reintroducing the synergic antithesis between freedom and control in search of new forms of compatibility achieved by adjusting re-selected mixes and contingencies;

c or, one may enter the postmodern, further destructuring the two terms and their relationships.

It is not difficult to place the various currents of post-Parsonian social and philosophical thought within these three ways of responding to the crisis of modernity. In terms of the morphogenetic theory, they may be classified as follows.

The first category includes the neo-communitarians, who commit errors of downward conflation, as they assume that freedoms can be regulated top-down by building new communities more or less impervious to general entropy; "communities are necessarily, indeed, by definition, coercive as well as moral, threatening their members with the stick of sanctions if they stray, offering them the carrot of certainty and stability if they don't" (Beckert 2006: 81).

The second includes the neoliberals, who commit errors of upward conflation, as they assume that the lib/lab synergy can still work bottom-up, expanding freedoms only to reassert the basic values and institutions of constitutional liberal democracy (see, for instance, Alexander 1994 and Habermas' 1998 constitutional patriotism).

The third are the neo-relationists, who commit errors of central conflation between freedom and control in that they define them through each other, "horizontally." They conflate agency and structure. Giddens' (1992) thesis on "pure couple relationship" is an example, as this relationship is 
structured by a rule which consists in the fact that the partners live together only as long as they derive individual satisfaction from it. Another example is Beck and Beck-Gernsheim's argument (1994) according to which the family institution becomes a social form made up of subjective preferences that work as social norms to themselves.

Indeed, the debate on the future of modernity is a mixture of these three "pure" types of response.

Amidst this debate, radical postmoderns emerge. They break with any humanistic tradition. As Luhmann put it bluntly and brutally (1995: 354), "man is no longer the yardstick of society." He argues that modernity cannot regenerate itself through any reference to human nature. Luhmann places freedom outside the system, in the so-called system environment, where the human subject may fluctuate as desired. Control is delegated to the autopoietic system. With the Luhmannian structural neo-functionalism, social theory reaches a decisive turning point, in the direction of a radically contingent relationism as to what concerns both freedom and control. Sociology proves itself ready to be placed in that crucible of postmodern thought that cuts all human ties between human freedom and social control. In this light, from my point of view, Luhmann appears simultaneously as the gravedigger of modernity and the lark of radical postmodernity, intended as a society that, after him, cannot distinguish the human-social from the non-human social (as in Latour's actor-network theory). This becomes the core problem of the postmodern world.

\section{Limitations and obsolescence of the lib/lab paradigm}

In late modernity, the freedom vs control dialectic meets structural and cultural limitations beyond which it may not go. What are these limits? We may summarise them briefly by stating that the late-capitalistic arrangement:

a identifies control with mere technical needs, or functional mechanisms, which should be managed by impersonal systems (regulated through negotiations between the state and the market),

b identifies freedoms using the yardstick of market freedom, thus generalising freedoms as processes of commodification,

c makes all associative spheres of social solidarity (i.e. non-profit-oriented actors, otherwise called prosocial private spheres, and civil economy) residual, allowed only to survive in the most marginal spaces of society,

$\mathrm{d}$ weakens the civil culture of the lifeworld, that is debilitates the civic commitment of people and their informal networks, through privatised and standardised forms of consumption and behaviour.

The lib/lab arrangement now stands on a process of ethical and cultural relativism. Ethics is not able to develop an adequate symbolic code for an internal differentiation capable of limiting the partiality of lib/lab ethics (i.e. 
institutionalised individualism) in the face not only of the great environmental, genetic, and technological challenges, but also to remedy the fragmentation of society and the loss of social integration.

This is the background against which advanced society, in the first few decades of the 21st century, no longer represents itself as the best of all possible worlds, but only as one of the many possible variations of one world that is infinitely "otherwise possible."

Indeed, many old problems remain unsolved, and others arise that the lib/ $l a b$ arrangement cannot confront. These problems have to do with:

- the crisis of the welfare state induced by the growth of freedoms guaranteed regardless of the negative consequences of private behaviours,

- the overflowing markets beyond national confines and other control apparatuses,

- the unregulated influence of the scientific and technological progress on bioethical and environmental issues,

- the increasing risk of amoral behaviour by subjects, against the increasingly mechanical nature of control systems which have by now long stopped relying on the purposeful, intentional motivations of subjects in behaviour areas such as drug taking and selling, environmental pollution, the diffusion of hazardous lifestyles, the perverse effects of the ICTs on people and their communicative relationships,

- the end of the Mandeville paradigm according to which private vices favour public virtues.

The end of modernity is revealed by its inability to achieve its promises. One realises that the lib/lab complex (freedom/equality) has made solidarity residual and continues to corrode the primary and secondary forms of social integration in lifeworlds. The lib/lab complex systemically empties the fabric of sociality. Society discovers that it is a powerful machine that turns life into merchandise. The freedom/control combination has taken on the abstract form of general intellect (of Marxian memory) that appears and materialises every day more in the globalisation processes implemented by the new communication and information technologies, the so-called infosphere (Floridi 2015) or, to say it with Teubner (2006a), by an anonymous Matrix of communication.

Within the winning model of Western modernisation, beyond the threshold where solidarity is radically eroded, the existence of sociability, as a sui generis reality, may no longer be assigned to freedom nor control, nor to a combination thereof, simply because the modern definitions of freedom and control implode.

The only alternative that remains is to conceive of sociological theory as the construction (and management) of paradoxes. Sociological paranoia becomes a keyword.

The dialectic between freedom and control becomes something else in relation to the dream of early modern civil society. The reconciliation 
between freedom and control appears increasingly desperate, because these two realities are kept separate under the representation of a society in which freedom floats in the environment of the social system governed by imperatives of control, without freedom and control being able to communicate. To go beyond, society needs a fundamental change in the guiding distinctions upon which social institutions are built. Sociology must choose whether to continue to reason within the old framework, or to consider an alternative.

\section{A relational approach to freedom vs control dilemmas in an aftermodern society: possible scenarios}

So far, I have argued that this symbolic code (lib/lab) can no longer act as the guiding code for the entire societal system. It becomes a mechanism for highly limited choices in specialised social sectors (particularly in the regulation of the trade-offs between the state and the market). I would now like to draw the theoretical implications of this way of interpreting society beyond modernity.

The overall premise lies in assuming that the freedom vs control relationship becomes an antithesis only in particular instances. The antithesis especially when synergic - is only one of the possible reductions of the relational dilemmas between the two poles. It lends itself to describing the relationship between state and market, but not relations within and between the other spheres of life. Generally speaking, a complex, multifaceted relationship arises between freedom and control.

When this reality takes on a new appearance, we enter the aftermodern world, where alternative relating processes emerge because the relationship between freedom and control may now be seen and enacted with many more degrees of contingency on both sides. This contingency is selectively reduced in different ways, according to the communication contexts, since these are relationally contested contexts. That this type of society presents new problems, and even immense risks, is intrinsic in its relational nature.

From a theoretical standpoint, a fact then emerges: that freedom and control are not simply two dimensions inherent in every social relation, but are social relations themselves, which must be conjugated differently in different social environments. ${ }^{2}$ We must define freedom and control as social relations, and do it without making their interaction with other relations and dimensions of social action - such as, for example, with solidarity antithetic or even perverse.

2 As I will explain later, stating that freedom and control are social relations in themselves means that AGIL must be understood as a complex of relations (Donati 2011a: 147-154), which in turn means that freedom must have its own internal controls, while control must have its own internal freedoms. Or, otherwise stated, freedom and control cannot be disconnected entirely but should be redefined as relations between the components of AGIL. 
Freedom not only stands outside control, but also inside it; freedom is a form of control and its source of justification. Control not only offers greater or lesser opportunities for freedom, but constitutes it, in the sense that it creates the various forms and degrees of freedom itself.

Freedom and control work together, and are not mutually exclusive alternatives. Instead, they are contexts and opportunities that develop one another. To see this, we must consider the freedom/control distinction as a complex relationship of social relations. But how is it possible to consider freedom and control as social relations?

1 As far as freedom is concerned, modernity has opened up freedom to the world of the possible by introducing new distinctions.

First example: by introducing the distinction between freedom "from"

(negative) and freedom "for" (positive), it has on the one hand expanded negative freedoms as demands for non-interference and on the other opened new horizons of positive freedoms as needs to achieve significant goals.

Second example: by introducing the distinction between procedural freedom and substantial freedom, it has on the one hand increased the possibilities for routine social relations and on the other made creative relationships of significant human intention possible.

The lib/lab complex, however, still sees the first side of these relationships almost exclusively. It mainly sees negative and procedural freedoms, while it has great difficulty in seeing positive and substantial freedoms. We need to rethink the sense of liberty if we wish to avoid nihilism.

This explains why the lib/lab sociology has observed freedom essentially in the form of the contingency inherent in "money" as a generalised symbolic means of interchange and communication, thereby making all social relations abstract and instrumental. But, in doing so, this vision has obscured the reverse processes, those through which new embodied, value-based, heavily intertwined and at the same time self-restricting social relationships have produced social forms outside those regulated by the lib/lab logic. Many sociological theories have not realised that the social relationship is an emerging effect of mutual action and have thus ignored the fact that vital associative worlds produce positive and substantial freedoms outside the state-market complex.

2 The same has happened for control. Modernity generated new distinctions of social control; that is, it created control as a social relation that can be played out in many diverse ways.

First example: by introducing the distinction between system integration control and social integration control, it has on the one hand built new norms that operate without resorting to 
actual human intentionality (checks through mere administrative procedures) and on the other opened up room for rules that instead require a rife intentional activation or adherence (when they are left to the discretion of the agent/actor, as happens in informal networks and in some digital media networks).

Second example: by introducing the distinction between hetero-control and self-control, it has on the one hand been able to construct impersonal apparatuses of social security and regulation and on the other explored the worlds of internal psychological conscience-based regulation.

The lib/lab complex favours the first side of these relations. It easily resorts to coercive system control towards people (using state or public apparatus, as happens to regulate the poor), while it has difficulty in recognising social control activated by subjects in their voluntary networks. Take, for instance, those social networks that give rise to protest movements, advocacy networks, or informal third sector initiatives, to name a few, which intentionally or unintentionally concur to exercise social control without being recognised in their contribution. This explains why most sociology has ended up seeing the social domain as that which negates the authenticity of the self, that is seeing society as a powerful machine that denies individual authenticity, rather than seeing primary and associative relationships as what makes it possible.

As I said, modernity tends to play out freedom and control as opposite dimensions, negatively correlated, but it still believes in the possibility of finding some sort of accommodation between them. It is assumed that if freedom is expanded along the refero axis (i.e. relation as an intentional-cultural reference), then control is reduced along the religo axis (i.e. relation as a structural bind), and vice versa. On the concept of refero I am referring to Weber's idea of social relation (Weber 2013 [1922]), while for the concept of religo, the reference is to Durkheim (1951 [1897]) (for more details: see Donati 2011a: 87).

Figure 1.1 depicts these two ways of understanding the social relationship as Cartesian axes in which the abscissa represents the relationship as a bond (religo) and the ordinate represents the relationship as a symbolic reference (refero). Modernity is understood as a society in which relations tend to take on a hyperbolic form, either in the direction of symbolic reference (called "refero") or in the direction of a constraint or bond (called "religo"), without connecting the two orientations, which correspond, respectively, to the theories of action and systems theories.

If the intentionality and the symbolic value (refero) are exalted, the structural link (religo) should be reduced to the minimum until it can be cancelled, as in the charismatic movements studied by Weber. If the structural link (religo) prevails, the intentional symbolic reference must be reduced to 


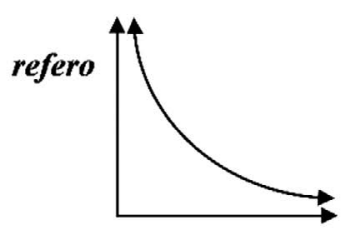

religo

\section{Legend:}

\section{refero $=$ symbolic axis (value and intentional references $=$ freedoms): e.g. competition as motivation and value}

religo $=$ functional axis $($ adaptive and regulative binds $=$ controls $)$ : e.g. constraints for social equality

Figure 1.1 The relational hyperbola of late modernity

Source: Author.

the minimum and eventually emptied, as in the idea of organic (functional) solidarity by Durkheim in The division of social labour, or, nowadays, as in cases of catastrophes and pandemics, when the axis of control (use of constrictive rules) prevails.

In late modernity, the tension between the two poles explodes. Compromises are no longer within reach. Instead of a system that proves unstable, but still thinks it can control itself to advance modernisation (this was the illusion of the cybernetic hierarchy of the Parsonsian AGIL), postmodern society assumes a hyperbolic configuration of itself, in which the antitheses find no accommodation or synthesis. The lib/lab antithesis (i.e. refero/religo) becomes a hyperbolic relationship in which the two terms of the relationship go on their own in an asymptotic direction (Figure 1.1).

Freedom and social control assume an asymptotic configuration, that is, they become "hyperbolic escapes" (Figure 1.1). The asymptotic development of control must expunge freedom in the system environment (thus outside institutions). The same occurs for the asymptotic development of freedom, which confines controls to its environment (thus only within system operations). In one direction, freedoms are placed outside the social sphere (thus outside social institutions), and in the other social control becomes only systemic (i.e. made of self-referencing and a normative automatism) and remains without justifying values. To understand this, take, for instance, the dilemma competition (marketisation as refero) vs social control for equality (welfare state provisions as religo).

The lib/lab complex thus ends up stretching all social spheres asymptotically towards "polar forms," either dominated by control (along the religo axis of functional constraints) or by freedom (along the refero axis of intentional-symbolic references). Private spheres (e.g. families) are further privatised, and public spheres (e.g. welfare service apparatuses) accentuate their surveillance and control character. 


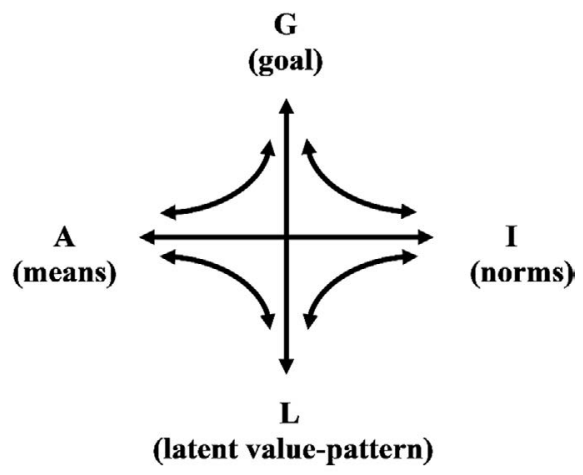

\section{Legend: \\ refero (L-G axis) = culture (symbolic and significant) \\ religo (A-I axis) = structure (adaptive and regulative)}

Figure 1.2 The hyperbolic relational nature of aftermodern society at its beginning Source: Author.

It is always improbable to find a balance point on the hyperbola in which refero and religo are connected to each other in such a way as to generate a significant emergent. This difficulty leads to the constant reduction of the social world to the Problematizierung of the world itself. It is no longer just about describing society as an antithetical oscillation between statu nascenti movements and processes of institutionalisation without that social institutions can correspond satisfactorily to the needs of the lifeworlds. The problem is that there is a dissolving of both social movements and institutions, at least in the sense intended by modernity.

I hypothesise that this formula of reading late modernity may be generalised using the relational interpretation of the AGIL multidimensional scheme to observe the transition to an aftermodern society as a relational society generated by modernity itself (Figure 1.2). The hyperbola of Figure 1.1 becomes specified when it is articulated within Figure 1.2.

The axis of the refero is interpreted as a relationship between latency (L) and purpose or goal $(\mathrm{G})$. The axis of the religo is interpreted as a relationship between means (A) and integration rules (I). The AGIL scheme, interpreted relationally, schematically represents society as a social organisation made up of the market (A), the political system (G), civil society or community (I), families and informal networks $(\mathrm{L})$ connected in various ways (Donati 2011a: 152-154). The letters A, G, I, L are intended both as analytical components of every social form and as concrete subsystems. Social forms exist in that they organise means (A) in relation to their goals (G), using norms and having values that justify the goals (L).

Now, the hyperbolic configuration of society that replaces any idea of an "organic" or modernising system implies that the four dimensions of social relations (goals, means, norms, values) tend to go their own way 
and to redefine relations with other dimensions based on mutant logics (Figure 1.2). The goals are no longer connected to the means, norms, and values; the means are no longer connected to goals, norms, and values; norms are no longer connected to goals, means, and values; values are no longer connected to goals, means, and normative standards. Each polarity tries to attract the others to itself without one dimension having an absolute and stable primacy over the others.

In the example of the dilemma competition (marketisation) vs social control for equality (welfare state provisions), their antithesis is transformed into many possible relational combinations of their respective elements (goal, value-pattern, norms, means) along the two axes of refero and religo. For instance, competition can assume the purpose of equality. Equality can adopt some market means. There are many different ways in which the value-patterns of competition and equality (as intentional purposes) can be combined with their respective means and norms, so to reconfigure the relationships between freedom and control otherwise.

If a group of people wants to create an organisation in which social solidarity prevails over any other motivation, it must provide itself with situated aims, norms, and means suitable to achieve its aims, cannot use the same means and norms as an organisation that works for profit purposes. Certain social cooperatives, NGOs, community foundations do this. If one of the latter, on the other hand, uses the same means and/or rules of forprofit activities, as certain social cooperatives or foundations at the service of multinational companies do, it will become something else.

Multiple social forms can arise. Then, we can see that the space of relations between freedom (refero) and control (religo) delineates the scenarios for new micro, meso, and macrosocial forms emerging in an aftermodern society.

These forms emerge from hyperbolic relations in which each dimension attracts the others to itself and redefines the relationships with them. This reveals the relational nature of refero and religo, that is, the fact that, being relations, they can take very different forms. In any case, their relationship, from a synergistic antithesis, becomes a complex of hyperbolic relationships in which one element seeks its own maximisation and reconfigures the other elements in relation to itself. In this hyperbolic configuration, the chances of having an indeterminate range of social relational forms increase.

Let us take the empirical example of couple relationships. In principle, if the couple relationship becomes "pure" (of pure intimacy, as though suspended in a normative vacuum), that is maximising freedom, or, otherwise stated, when the love relationship is reduced to the axis of the refero (L-G), then the institution (the religo of the couple, i.e. the relationship A-I between means and norms) is brought to an asymptotic minimum, which means that the institution no longer controls the couple's life. How will this configuration evolve? To another institution? Various possibilities are open, which can be seen through morphogenetic processes (see Chapters 4 and 5). 
On the other hand, couples exist who have an opposite characteristic, that is, they maximise the axis of the religo (A-I) by privileging the issues of means and norms (see the diffusion of premarital contracts in the United States and elsewhere). What happens if, at a certain point, the axis of freedom comes into play, not to undermine or renegotiate the contract that is legally binding, but because one partner asks the other to ignore the contractual rules or forget them to affirm feelings of love instead of legal norms? In this regard too, developments are a question of how social morphogenesis will evolve.

We could imagine the most different situations and the search for the most different social relationships. What is important is to understand that the relational society opens Pandora's box and, in order to find new solutions, it needs to resort to another symbolic code than the modern and postmodern ones.

To describe society as a relational-hyperbolic tendency of social forms is clearly an alternative to the description based on the functional primacy of one of the four functional prerequisites adaptation, goal attainment, integration, or latency. The functional primacy of one of the four poles is no longer possible (as Parsons and Luhmann still believe) for the entire society.

\section{Summary}

In conclusion, the more society becomes aftermodern, the more each relationship (each sphere of social relations) tends to be based on its own guiding distinction (its own principle of operation) in such a way that freedom and control are not seen in an antithetic contraposition, but combined in differentiated modes, by changing their own elements and their reciprocal relationships.

Perhaps, by adopting a relational paradigm, we can see how the norms of freedom and control lie neither simply in the individuals (as "abstract" subjects) nor in systems (as normative structures) but in social relations when taken seriously for what they are: fully emergent effects from reciprocal actions between social subjects.

Herein lies the novelty of civil society, which beyond modernity no longer coincides only with the democratisation of the political system, but above all with the possible emergence of collective subjects that express new processes of civilisation.

The political expression of this project can be called "societal citizenship," intended as distinct from nation-state citizenship. Societal citizenship is produced by forms of social governance instead of political government. It is built as a co-growth of freedom and control within social networks acted by relational subjects, through distance relationships between civil society and the state, rather than as an ascriptive emanation of the nation-state (implemented, as in modernity, through the principle of progressive inclusion of the population in it). 


\section{The emergence of aftermodernity}

Social relationality is the new glue of society, not the state per se, or an abstract normative system, or functionalist adaptation systems. It may perhaps be called "the political" (le politique) of social exchanges, as opposed to "the politics" of the political party system (la politique), according to the distinction made by Caillé (1993). But only if we see that the political stuff of society consists of its "relational glue," and if we can observe it in an adequate way, we can realize that "the political" has become simultaneously more global and more local, meaning that it has spread throughout all relational dimensions of society and at the same time has differentiated within each societal sphere, according to autonomous intersections (nexi) between freedom and control. In the 21st century, society will be able to manage "the political" only as a form of post-liberal as well as post-labour relationality unknown to the moderns. 


\section{Overcoming the market/ state binary code}

\section{How to explain the recurrent system crises?}

It is an established fact that modernising societies are subject to recurrent system crises. I take the financial economy's crisis that broke out in September 2008 as emblematic for the way in which many other system crises are faced. I am referring to the industrial revolution 4.0 driven by new robotic technologies and artificial intelligence (AI), the economic and social crisis due to the Covid-19 pandemic erupted in 2020, and others.

To get to the point, most theories converge on the idea that world society is bound to face a future bristling with risks, uncertainties, disorientation, and even chaos (in the technical sense of the word) without hope of solving system crises. Only caps and temporary remedies can be provided or let crises run their course. The dictum by Luhmann (1976) that "the future cannot begin," means that societal systems must accept a condition of permanent crisis. Where the term crisis has the meaning of a critical event that stands for (or is expected to) leading to an unstable and dangerous situation affecting the whole society, but also as a test period for further adjustments, or an emergency event, a turning point in a disease that sooner or later we will come out in ways that we cannot know in advance.

In this chapter, I am interested in analysing how modernised societal systems react to recurring crises. Their reaction consists of a game between the political system and the market. In the end, they must take note that the political power can enforce very few limitations to economic systems, and economic systems cannot expect politics to solve their problems. Their exchanges are very limited, only contingent, merely functional, and cannot meet normative imperatives beyond economic and political action. Ethics is turned into an exaggerated steering mania, which proves to be practically ineffective when challenged by real incidents (Luhmann 1997: 50).

The measures adopted cannot solve the crisis for a number of reasons.

First of all, all the remedies remain within the "economic-political system," what I call "lib/lab" configuration. My argument, then, is that if we want to avoid a permanent crisis, then remedies have to break away from the self-referential logic of economic-political systems. 
Secondly, the ethics that is called upon to correct the markets' malfunctioning and state's failures has no credible sociological foundations, since neither the market nor the state are sources of ethical standards. If ethical corrections are to work, one needs to think of a different way of organising society. Such a new setup (i) has to be capable of allowing for the emergence of collective relational subjects (viz. "social environments" for the economic and political system) that can generate and adopt certain ethical standards of conduct and uphold them in economic-political systems and (ii) has to meet such a condition in a structural manner and not by way of an occasional voluntary commitment. Luhmann (1995) would say that this is not possible, because - in his view - society's multiple spheres cannot in any way influence one another, least of all exchange ethical services. I propose to meet the challenge of proving that this is as possible as it is necessary, if we want to avoid permanent crises.

\section{My explanation}

What are the mechanisms that make the lib/lab society unsustainable? Here I would like to analyse the problematic aspects of these systems and subsequently verify whether there can be a societal configuration that can overcome their limitations. Let us first look at intrinsic faults of the lib/lab setup.

According to the lib/lab approach, the structure of society is basically an interweaving of economics and politics with respect to which "the rest" - as a private affair - is seen as marginal and irrelevant to the common good and citizenship. The lifeworlds are conceived as merely "private" spheres. But, from a sociological point of view, what lies outside the state-market pair is not insignificant for the achievement of the common good, for citizenship, and for the workings of both market and state. If the lifeworlds are conceived as mere receptors of state and market decisions and activities, and therefore devoid of social subjectivity, the lib/lab system falls into a chronic crisis of social integration it cannot remedy.

To put it another way, for the lib/lab system, there is no alternative to the combination of liberalism and socialism. A champion of this approach, Ralph Dahrendorf (1994) has defined citizenship as a gift granted (octroyée) by an enlightened political élite that extends the social rights guaranteed by the state to increasingly large portions of the population to make available to them the provisions offered by the free market. Such a societal configuration refrains from tackling the social integration problems due to the erosion of social relations induced by capitalism and state-induced population passivation. Even though one may agree that society's system planning is not a workable regulatory response, still it is clear that lib/lab systems generate increasing social integration deficits - the so-called modernity pathologies (Habermas 1984) - for which they provide no remedies.

The world system (or globalisation) is the outcome of this worldwide societal lib/lab structure. What determines the crises is a kind of "economic logic," an evolutionary model which is supposed to bring us to "progress," that produces 


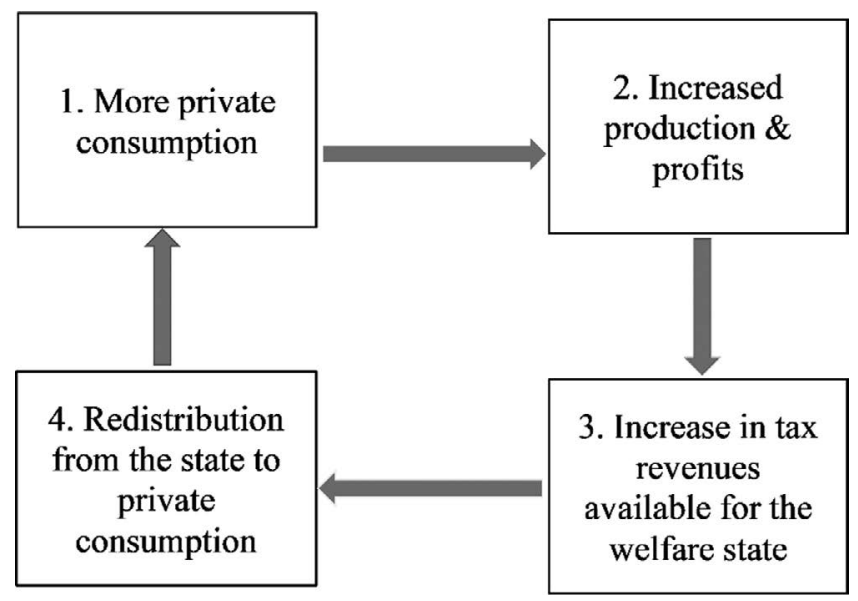

Figure 2.1 The economic logic of a lib/lab society

Source: Author.

unexpected effects, side effects, and negative externalities which erode the civil society on which the lib/lab system is based. What is this logic about?

Let me summarise it in Figure 2.1. The economic logic I am talking about consists in using political power to favour consumption, especially private ones (Box 1), which in turn will fuel productivity and profits (Box 2), in such a way as to be able to increase the tax levy (Box 3) that allows the welfare state to redistribute more financial support to the population necessary to increase consumption (Box 4). The rest is irrelevant. Banks and financial systems serve this logic.

Such a system logic, with all its internal mechanisms, cannot be extended over certain thresholds, because great social problems arise once certain economic growth levels are exceeded. In particular,

1 Consumerism generates a broad range of pathologies in the human condition of many people: consumption needs are artificially induced, and media technologies are misused both by producers and by consumers.

2 The social inclusion model that is supported by this logic (founded on a simple extension of the typical 20th-century welfare state) makes beneficiaries ever more passive and produces distorted effects: it creates various traps (the poverty trap, the crystal roof limiting women's social mobility and distorting equal opportunities on the basis of gender, etc.) and above all immunises individuals from social relations.

Many will point out that there are no alternatives to the system logic I am talking about (Figure 2.1) because (i) if you curb consumption, you also stop economic growth; (ii) if you cut social expenditure (the welfare state), you create poverty. 
What shall we do then? The proposals put forward are centred on introducing two kinds of correcting tools:

1 Putting "more ethics into the market," as proposed by some, in the hope of making actors more responsible; two examples of this are business ethics at the production stage and a fairness ethics in the distribution of goods; such proposals are especially aimed at correcting the lib side of this setup.

2 Extending citizenship, as proposed by others, to make it more inclusive to embrace the weakest social segments of the population, in order to reduce poverty and social problems; such proposals are especially aimed at correcting the lab side of this setup.

I note that such corrective measures do not modify the system logic of lib/ lab configurations. As generous as the above proposals may be, they do not stand many chances of succeeding because it is the lib/lab system itself which makes them ineffective. The system continues to work in such a way as to be functional to a moral order centred upon individual, instrumental, and utilitarian values and criteria. Though sensitive to the need for personal honesty and greater social justice (in the form of equal opportunities), these values and criteria fail to meet the need to create a civil society capable of generating a social morality that supports sufficient cohesion in the networks of relationships between people. Economy refuses to consider ethics as an autonomous variable of economic action (and function). In the long run, a system that gives absolute primacy to the means, tracing back to them the other components of social relations (the integration norms, the goal, and the pattern-value: see Figure 2.2), is self-defeating. If the societal configuration is to generate a social integration in the lifeworlds, which is sufficiently synergistic with the system integration (market/state), the lib/lab logic must be radically modified.

\section{Should we adapt to a Darwinian evolution?}

The lib/lab vision of the world system brings with it the idea that society develops according to progressive evolutionary tendencies. The processes do not tolerate any restrictive, external regulations of the lib/lab logic (as summarised in Figure 2.1). The only regulations this logic can endure are the functional ones, which is functional to its own reproduction.

A functionalist approach, anyway, can only determine the same problems again and again. Luhmannian neo-functionalism turns to be just "another way," only outwardly non-ideological, of describing the commodification of the world and an evolutionary adaptation of the whole society to such commodification processes.

Basically, the functional version of the lib/lab model proposes us to live in a society that adapts to Darwinian evolutionary laws, lacking any 


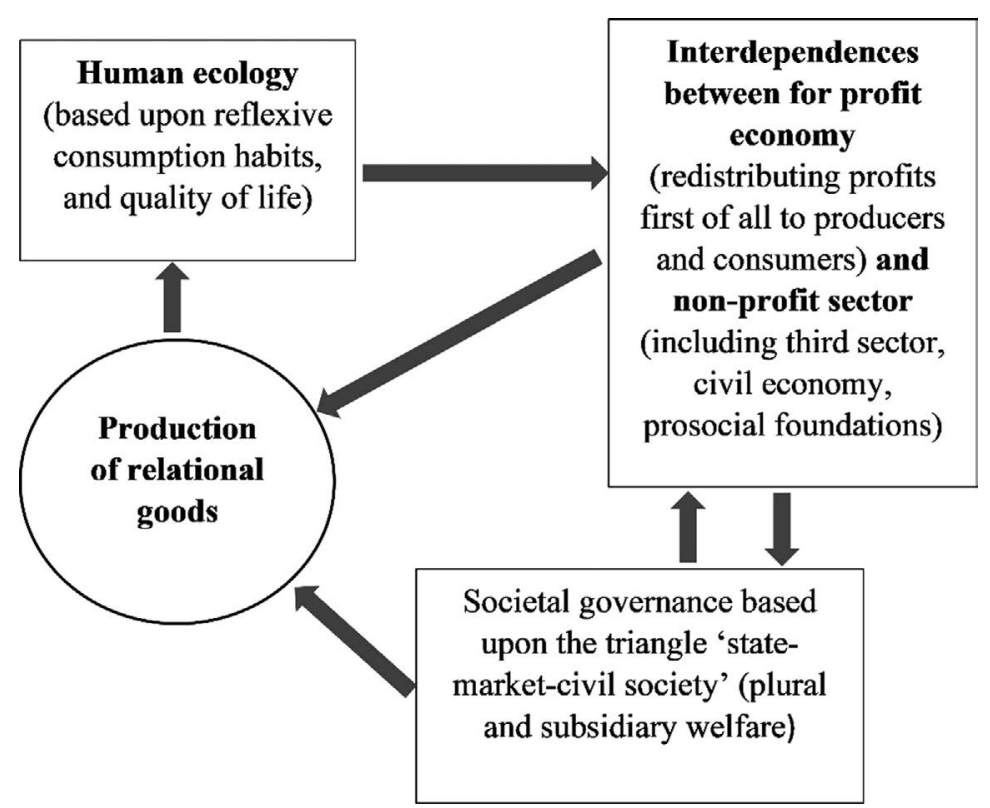

Figure 2.2 The economic logic of a relational society

Source: Author.

finalism and pushed by its competition and survival skills. Cooperative behaviours are possible, but nevertheless they remain marginal. This is the (Luhmannian) world system of globalisation.

There seem to be no alternatives to this state of affairs. Utopias have fallen. The word is given to the stock exchanges. George Soros (2000), the international magnate, has pointed out that financial markets' workings follow their own "reflexivity" (or reflexive rationality) marked by evolutionary mechanisms, which are self-referential and have uncertain outcomes. These mechanisms are rooted in the particular reflexivity of economic actors who discount the future. They shape reality (i.e. what actually happens in society, not only within markets) through investments that anticipate the future and pre-empt future reality in the shape desired by financial operators. Reality is transformed through the financial operators' own "reflexive truth."

This is another version of a society evolving under the primacy of the adaptive function guided by money as the engine of evolution. Money is the g.o.d. - the generator of diversity - of Darwinian evolution. The question that arises is whether it is realistic to think that society can evolve as a financial market with its so-called reflexive logic promoting an evolution without finalism. Clearly, society is not a stock exchange. There are other types of reflexivity that shape society. It is these "other" forms of reflexivity that can get us out of the lib/lab systems' own chronic crisis. 


\section{No alternatives to an evolution without finalism?}

Can we think of alternatives to the functionalist and evolutionist model I have been discussing? I think that the world is in need of a post-functionalist, indeed an aftermodern development model, which goes beyond the functionalist model hitherto considered the theoretical and empirical infrastructure of Western modernity (Davis 1959).

However, a word of caution is needed here. Functionalism cannot be overcome by a backward-looking humanistic view, unable to match the competitive skills of functionalism. It has to be a humanism proving capable of taking functionalism into account while overcoming its limitations.

Such a post-functionalist development configuration or logic ought to be able,

1 at a macro level: to reduce system determinisms by lib/lab institutions, in order to avoid commodification mechanisms,

2 at a meso level: to favour organisational networks capable of selfsteering,

3 at a micro level: to change consumption habits and lifestyles through reflexive consumption and according to more sustainable values in the lifeworlds.

It seems to me that such changes are not possible within an economy whose ruling principle is the GDP growth imperative. They become possible, though, as soon as one takes on board the fact that GDP has been a useful well-being parameter for developing countries with quite a low average income and with widespread poverty problems, but it becomes hardly significant for societies that have reached a certain well-being threshold. Starting with the latter countries, GDP has to be replaced by other parameters of measurement and evaluation of progress that refer to Gross Domestic WellBeing (GDWB) (Stiglitz Report 2009).

A sustainable lifestyle does not mean a "poor" economy that reduces aspirations to a qualified well-being. It does not mean, for instance, a mere deindustrialisation or entrusting health and school services to imaginative "community" solutions, nor does it mean rejecting technology. It does not mean going back to a naively "naturalistic" way of life. These are utopias without any hope. A different economy is made possible by a different notion of well-being and of happiness with relational and not merely materialistic connotations (Diwan 2000). This is suggested by the "happiness paradox," according to which the well-being - beyond a certain income threshold does not grow with the increase in available money, but instead with significant human relationships.

The recurring crises need to be addressed with a model of sustainable development (see Figure 2.2). If we want to pursue a human ecology (i.e. preserve the ecosystem), reflexive consumption and attention to the quality of life are required. The processes of production-distribution-consumption of 
goods and services should assume a relational configuration by promoting a synergy between profit and non-profit sectors, which means a modification of the logics of both. The for-profit sector must become socially responsible, and the non-profit sector must become more efficient and effective with full autonomy with respect to the other sector. This latter target is certainly difficult to pursue. Take for instance the case of health service. International evidence indicates that non-profit organisations (NPOs) are likely to be more attentive to service quality but less cost-efficient. However, this is not always true. For example, researches on the care sector in Italy show that NPOs can be as efficient as other organisations. What is even more important is the fact that the efficiency of these organisations is positively correlated to the production of relational goods, highlighting the importance of intrinsic motivation compared to the profit motive (Destefanis and Maietta 2015), as well as having greater value for a caring democracy (Zubero 2018).

The rule of the welfare state shall be gradually replaced by a societal governance, featuring a "market-state-third sector" triangle (plural and subsidiary welfare). Societal governance should operate reflexively both on consumption and on the differentiation of different markets (for profit, nonprofit, civil economy, etc.) aiming at producing relational goods.

These changes indicate the birth of another type of system configuration, based on a relational economy that evaluates progress in terms of GDWB rather than in terms of Gross Domestic Product (GDP), as illustrated in Figure 2.2. The figure outlines the economic logic of the relational society through a circular sequence: human ecology can be pursued through the interdependence between profit economy and non-profit sectors, so that the societal system governed by a joint partnership between state, market, and third sector can promote the production of relational goods on which human ecology depends. Furthermore, it is indicated that the interdependence between the profit and non-profit sectors itself produces relational goods regardless of system regulations.

It is important to emphasise the role of the social spheres bearing a development model that connects the economic to the social after modernity has separated them. I am referring to those economic realities which are partly non-profit and partly managed to producing profit for ethical purposes. The non-profit sector is made up of third-sector organisations, such as civic associations, voluntary organisations, NGOs, and civic foundations. The novelty is represented by the so-called civil economy companies, which aim to do business ethically through a cooperative and solidarity organisation both between producers and between producers and consumers. These are entrepreneurs and managers who intend to produce profit with social responsibility, in view of a sustainable economy, and allocate most of the profit for prosocial purposes.

Within the third sector, we find a vast world of social cooperatives, social enterprises, voluntary associations, ethical banks, various forms of microcredit, fair trade, NGOs, community foundations, and multiple forms of 
enterprises which we call civil. Such bodies create their own financial markets, such as the the KIVA project in the United States, SASIX (South African Social Investment Exchange) in South Africa, Bolsa de Valores Sociales y Ambietais (BVS\&A) in Brazil, the Asian Impact Investment Exchange (IIX) managed by the Social Stock Exchange Asia (SSXA) in Singapore, GEXSI (Global Exchange For Social Investment) in the United Kingdom, MYC4 in Denmark, and Social Stock Exchange Ltd. in the United Kingdom. Others have proposed to create a "social stock exchange," aimed at managing "social and welfare business," which would become an integral part of a horizontal subsidiary setup between the welfare state and an active welfare society. This might happen by setting up a sort of Alternative Investment Market, whose financial instruments would be shares (issued by low-profit enterprises and non-profit social enterprises) and debt bonds (equally issued by for profit and non-profit bodies).

Such new enterprises as low-profit limited-liability companies and community interest companies, as well as new ethical banks, can produce a different response to the system crises, not merely by adapting themselves but by giving moral standards priority in economic and social action and by being able to modify life, investments, work, and consumption styles. Compared with traditional capitalist enterprises, such enterprises have a number of peculiar features: they are bound to produce relational goods (and more generally intangibile goods), and they show greater flexibility and value lateral social mobility rather than upward or downward job mobility.

Not only is the influence of all these actors growing, but above all such spheres operate as an engine of a civil society that is alternative to the typical capitalist market underpinning the lib/lab setup.

As Manterys (2017: 86) writes,

Any change in the spirit of aftermodernity and building a relational civic society implies the emergence of new relational structures, which sustain the autonomy of individuals while adding capital to their relational, and not individual, aspect through "valorisation" of the new social forms, which are usually placed in the "third sector" (Donati 2015a: 99-105). In other words, the emergence of a new social formation is at the same time an introduction of a "third actor" and implementation of relational imperatives mitigating the topdown oppression of the authority of the state and the instrumentalism of competing for precious resources, and through this allowing fuller civic participation and the liberty to form symmetrical and non-instrumental relations.

\section{Rethinking civil society beyond modernity}

The recurrent crises of the lib/lab configuration have highlighted the difference (a real splitting) between the old and the new civil society, that is the one that still reasons in terms of liberalism and socialism and the new 


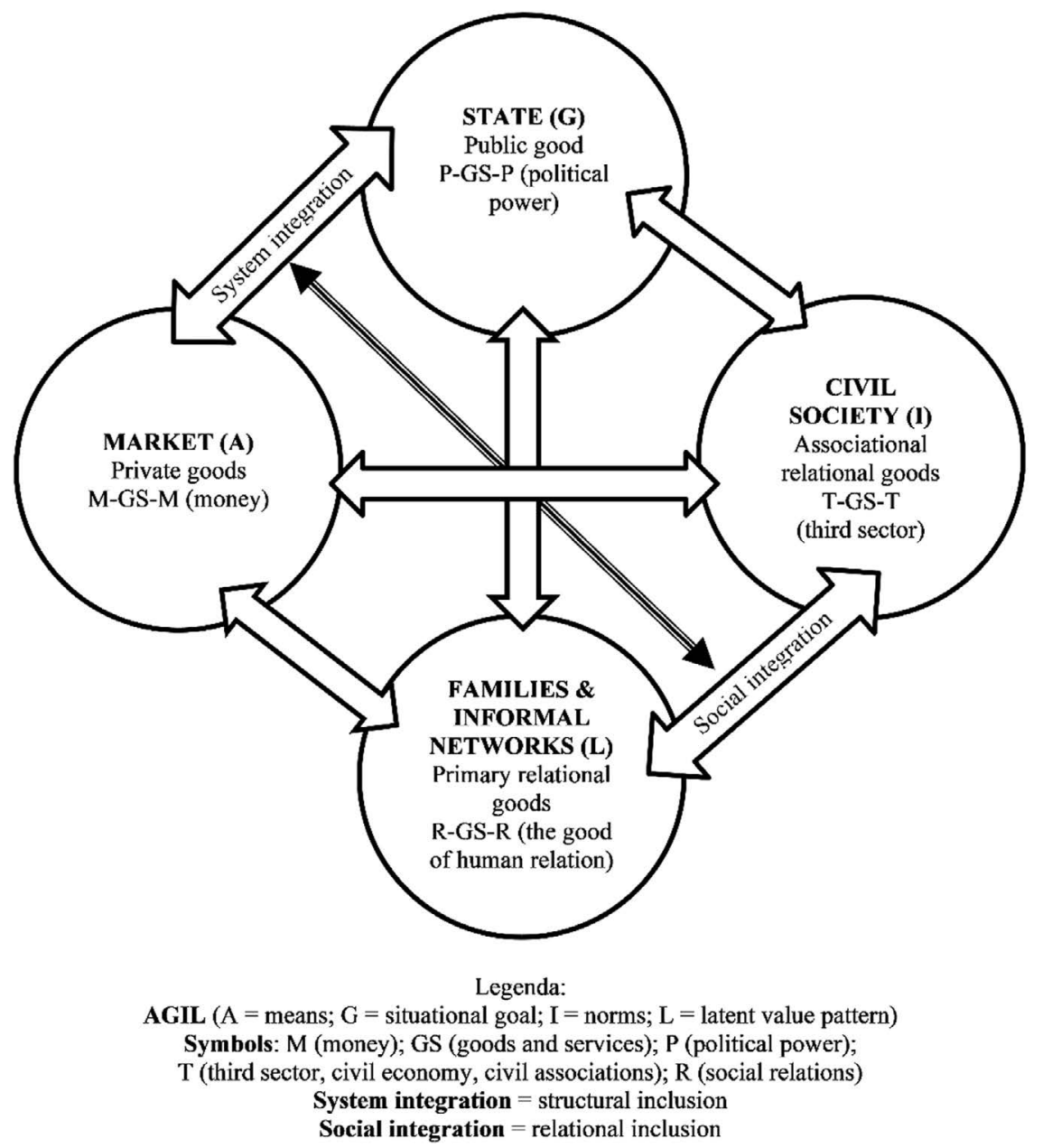

Figure 2.3 Aftermodern society as a societal network intertwining system and social integration

Source: Author.

one that instead brings out a distinct third pole, distant from the other two, capable of redefining them. We may have reached a turning point between the old and the new civil society.

The new societal configuration (as outlined in Figure 2.3) does not completely erase modernity, but sees the modern lib/lab setup only as a particular case, that is as a way of operating (i.e. organising economy, politics, etc.) which is only applicable to ever more limited action areas. The new setup is not characterised by a logic of dominance of a pole (market or state) over 
the other or by commercial negotiation logics between subsystems, but by a network-like logic which is forced to make the different societal spheres more co-operative, or at least to follow a mutually non-destructive competition logic, within a global sustainability project.

To implement such a setup, one needs a relational configuration that modernity was unable to tolerate, because it was overwhelmed by cultural movements conceiving of modernity as a way to immunise people from sociality.

As outlined in Figure 2.3, on the one hand, the old civil society is still amongst us, tending to subject every good to the sequence by which money is invested in goods and services (GS) which in turn are used to make more money (M-GS-M). Actors, that is, invest money in a good they have no need for, but which is only instrumental to making more money. At first, they attribute to that good a monetary (functional) value and then trade it to make more money. It is important to understand that this mechanism presides over the whole lib/lab system. The state also uses it in its relationships with the market. The state uses the market to get the money to pay for public welfare, which in turn is the source of votes, the political system's own money. In this context, civil society is identified with the market.

On the other hand, a new civil society is emerging, which is identified with real economy. In real economy, in contrast with the previous case, the good is evaluated in itself and money (also in forms different from currency) is only used by actors as a tool to acquire the goods and services they need [according to the sequence GS-M-GS]. A good is translated into the money only in order to obtain another necessary good. Money is embedded into social relations, and its different forms depend on which social relationships are at stake.

Rethinking civil society means understanding whether, and how, it is possible and necessary to shift from the M-GS-M sequence to the GS-M-GS sequence. This shift modifies modernity's own view. At the core of this view lies the relational nature of goods and services. Indeed, if it is correct to say that the distinctive feature of a modernising economy is to erase the relational nature of goods/services and economic processes, the building blocks of a new economy will be precisely the new needs for interpersonal and collective relationships. It is not by accident that we see gifts - and the enlarged circuits of exchanges based on reciprocity - coming back into so many social spheres and in many different forms: as redistribution, investment, free voluntary work, civic action, and also means of production. From a sociological point of view, gifts and reciprocity exchanges point to the pursuit of social bonds and to the need for social relations to be forged to cement the sense of community.

The key element of the distinction between modern and aftermodern society is the fact that the latter is confronted with the need to produce a variety pool of options in goods/services production and consumption, in life styles, and in welfare measures which cannot be evaluated in terms of a merely functional monetary equivalence. This pool must respond to existential needs of the life-worlds, must replenish primary natural resources, and above all must do this through the creation of common goods, by which I mean relational goods. 
This results in the rise of a new Zeitgeist. Whenever we say that future society will have to be inspired by the ethical criterion of sustainability, everyone has different things in mind, but the basic idea is the one for which instruments, such as finance and technology, must match human needs, and not vice versa, without compromising resources for future generations. It implies that the means must be used only as means, and not as autonomous ends or objectives, and be legitimised by values of relational well-being and with appropriate regulatory rules.

I summarise the distinction between modern and aftermodern setups as follows:

1 Main features of a "modern society":

(A) The preferred symbolic medium of interchange is money intended as currency (which decides the value of all other factors, in particular production, labour, and technology).

(G) The goal of society is to increase the GDP, and the only constraint imposed on money is that it provides more money; money is an end in itself, because of the functional culture which makes all goods and services subjectable to monetary equivalence.

(I) Companies have no broader social responsibility than that strictly associated with their own employees and customers.

(L) The value motives of social action are individualistic, instrumental, and acquisitive.

2 An "aftermodern society", on the other hand, is featured as follows:

(A) Money, as symbolic medium of communication and interchange, can take many different forms, monetary, and non-monetary; the medium of currency as the privileged symbolic medium of interchange leaves room to other forms of money understood as entitlements to goods and services provided not only by the state, but also by the market, the third sector, and the community (Donati 2001: 189-197); the distinction between monetary and non-monetary means is assessed based on their specific and differential contribution to the real economy, in which many goods and services do not allow for monetary equivalents.

(G) Society's goal is to maximise GDWB, and for this purpose, money (in its various monetary and non-monetary forms) is subject to constraints that must support sustainable relational contexts; the different forms of money are incorporated into social relationships that decide on its value and the type of exchanges for which it can be useful.

(I) Enterprises take on an external responsibility to the community's stakeholders; corporate social responsibility is extended outside the company; profits do not only or entirely go to shareholders, but must be shared with the stakeholders; social responsibility is also 
broadened with regard to employees with forms of reconciliation between work and family, through relational contracts as well as corporate citizenship.

(L) Value motives are relational, that is oriented to pursue a relational well-being that is generated by relational goods and in turn regenerates them.

Therefore, aftermodern society is characterised by an observable multiplication of forms of money, labour, and capital. The types of work multiply due to technological changes. Capital is considered not only from a financial point of view, but also as a political, social, and human capital. This entails a multiplication of contracts, in addition to the classic ones, towards forms of relational contracts necessary to pursue an economic objective embedded in a relational social context (Donati 2001: 181-188).

\section{A relational society needs a "relational state"}

Since the 1990s, I have launched the idea of the "relational state," whose rationale is based upon a relational theory of society. The traditional welfare state should be replaced by a new societal configuration characterised by the fact of being shared, associational, and relational within and between the different social spheres. A progressive society needs a truly responsive welfare state that builds the capabilities of all relying upon a relational work: services that value and build on relationships. This is the relational state.

A form of welfare that understands that a social issue stems from a factual configuration of the social relations between people and not only in their individual good or bad will, that loneliness makes people sick and eventually kills, that personal insecurity or poverty depends on the lack of a reliable social network, that people need a social network to find a job when most of jobs are never advertised, and that people need a community to address the problems of violence, depression, and anxiety.

To pursue a relational society means connecting high system integration (at the level of macro-institutions) with high social integration (at the micro level) going through forms of reticular associations at the meso level. If we criss-cross the two aforementioned forms of integration, dichotomising them for simplicity in high and low, we get four types of welfare state configurations.

- Type 1 (low system integration and low social integration): this is a society where a fragmented market welfare (lib) prevails (e.g. the United States).

- Type 2 (low system integration and high social integration): these are societies characterised by a stratified and segmented residual welfare left to primary social networks (e.g. underdeveloped countries). 
- $\quad$ Type 3 (high system integration and low social integration): this is a societal configuration characterised by the traditional institutional welfare state (lab) (e.g. countries governed by a form of socialism).

- Type 4 (high system integration and high social integration): in this case. the societal system is characterised by an institutional plural welfare; the whole configuration is that of a relational state supported by a relational society (e.g. emerging policies and practices in evolving postlib/lab systems).

The relational society is one where the political institutions put in practice the principle of active subsidiarity, in its various forms - particularly in the circular form between the political system and civil society - and by that way they ensure high social integration in civil society. The Province of Trento in Northern Italy (Malfer 2019) provides such an example. Its political system operates through an extended network of social cooperatives and a regular partnership between public and private organisations through intermediary bodies.

A relational welfare state is not just an idea. It is the political form of a relational society. Its basic principle is to provide better levels of basic welfare through the building of rich social networks that can empower people through forms of cooperation among the four basic sectors in order to produce relational goods.

The relational state is a way to design services aiming at empowering people and families in order to face many difficulties in day-to-day life. Families have the potential to change their own lives. The relational state empowers civil society by adopting a relational vision of all social issues and their possible solutions within a framework that promotes relational generative mechanisms (Donati 2015b). It provides the framework for those at the front line to create new relationships with people that support fair and sustainable social transformation.

A relational approach defines not the goals assigned to people, but the way through which people can achieve their inner motivated goals in relation to significant others. Relationships are the glue that keeps people together and the relational state can build public services that foster good relationships. For instance, a service can create opportunities for those seeking work, by providing someone to vouch for you, to support you, and be reflexive with you. It will promote the building of a social network around you within this framework, including the support for the small businesses that will drive job creation.

According to Cook and Muir (2012), there are four areas where scholars should set out their ideas more fully.

The first is how people working in public services can be supported to acquire the skills required by the relational state. Mulgan (2012: 10) argues that the skills and capabilities of people working in a relational state will be different to those in the "delivery state": "the ability to empathise, 
communicate, listen and mobilise coalitions of citizens and professionals to achieve social goals." For example, he suggests that we make healthcare more like education, deliberately aiming to raise the skills of the public through, for instance, courses or e-tutorials to support people with diabetes and dementia. According to this perspective, we need to identify new roles that staff will play in 21st century public and private services ("catalysers," "navigators," "brokers," "storytellers," "resource-weavers," "activators") as part of a process of supporting citizens to be co-authors of their own lives. Social workers should be trained in learning new methodologies of relational work. Supporting professionals in acquiring these skills and building effective relationships is a key challenge, but one which professional bodies, universities, and service providers are not yet well set up to meet. My relational perspective emphasises the need to share learning across welfare professionals and informal caregivers and to ensure that those engaged in relational services, such as health care assistants, social care workers, and classroom assistants, become keen on how to do a relational observation $(\mathrm{O})$, a relational diagnosis $(\mathrm{D})$, and a consequent relational guidance $(\mathrm{G})$.

ODG systems are based on the sequence: relational observation $(\mathrm{O}) \rightarrow$ relational diagnosis $(\mathrm{D}) \rightarrow$ relational guide $(\mathrm{G})$. The principles on which ODG systems operate are as follows: (i) it is a question of defining the social problem and its solution in terms of relationships (e.g. if the problem is unemployment, it is a question of defining it in terms of relationships and look for the solution in modifying the relationships that generate it); (ii) the relational guide consists in activating all those involved in the problem by building a social network between them, and then making them interact spontaneously, while a team coordinates the subjects so that the interactions are oriented to cooperate in order to produce relational goods (for more details, see Chapter 5 in Donati 2011a).

The agency is made by all the parties, as in an orchestra or a sports team, where everyone follows a cooperative standard that is used to continually regenerate a non-hierarchical and non-individualistic social structure and consequently modifies the behaviour of the individual subjects, who are driven to generate relational goods.

Now let us see some details to explain the acronym ODG. (O) Relational observation aims to define the problem as due to a certain relational context. Therefore, it favours the meso level (in which relational goods can be produced). (D) Relational diagnosis aims to define the satisfactory (or not satisfactory) conditions with respect to the effects produced by the relational context. (G) Relational guidance aims to modify the social context so that it can be mastered by the agents/actors in order to generate relational goods.

A second area where more development would be useful is on how the emphasis on relationships will intersect with an outcomes-focus. Social relations should not be conceived as "objects" of the same kind of concrete or material outcomes, but nevertheless we can speak of relational outcomes. Mulgan (2012: 25) writes, "Some of the goals of government have to be 
concerned with outcomes - fewer families in crisis, for instance, or better survival rates in hospitals. But others should be relational...." This sentence seems to trace a functional distinction between goals as outcomes and goals as relations that is inappropriate, because the former have a relational constitution like the latter. Outcomes are not the opposite of what is relational: in fact, we can talk of relational outcomes as a new configuration of relations leading to the resolution of a problem. Relational outcomes can be measured, although with different methodologies in respect to quantitative performances. The complex links between social relationships, outcomes, and culture can be investigated within a normative framework that can handle unbound social morphogenesis (see Chapter 6).

A third area is concerned with the discussion about the practices of devolving funding down to the individual level through personal budgets and pupil premiums and how they will intersect with the emphasis on relationships. The literature on social work has highlighted potential tensions between individualised funding and a therapeutic social work based on valuing relationships. Personalisation does not (and should not) mean individualisation. To reduce the human person to an "individual" means to embrace an impoverished ontology, namely one that fails to accord sufficient weight to the primordial and existential realities of human interdependence, inter-being, and symbolic interaction. This is not to deny the importance of personal choice and control in life planning, but rather to argue that choices and control are best favoured within an ontological framework in which inter-being, sociability, and the socially reflexive nature of the self is at the fore. The relational state should operate through a relational reflexivity and refer to relational subjects.

In the end, relational state situates the relations between the public and private sectors, between the state and civil society, in the sphere of coresponsibility, partnership, and co-production (Mendoza and Vernis 2008). The relational state is a perspective based on the thesis that changes affecting the economic and political structure in recent decades have transformed the roles and capacities of social agents, above all in public and government sectors. The globalisation of the economy can transform the traditional welfare state model into a relational state model, above all in its role as public manager. The organisational model to which the relational state belongs is that of the social entrepreneur, capable of creating and managing complex inter-organisational networks in which public, private, and civil society organisations play their part. This new relational model of governance relies more on decentralised civil society initiatives, media exposure, and business self-regulation than on active state intervention. It adopts the principle of subsidiarity together with the principle of solidarity, which means to overcome the defensive and restricted interpretation of subsidiarity as mere "devolution" or "let people do things by themselves." The relational state is a modality of enforcing an active and promotional interpretation of subsidiarity as "a way to help people to do what they have to do." 
Relational thinking challenges the assumption of the prevailing economic paradigm that it is best to pursue economic growth at whatever social cost, and then pick up the pieces of poverty and broken families afterwards through tax and redistribution policies. An alternative approach, which puts relational priorities first, would seek to protect families and communities while pursuing a Gross Domestic Wellbeing and thus avoid the need for subsequent redistribution and social intervention. Changed priorities in schools would mean no longer aiming to maximise the potential of each child expressed in terms of economic or individual achievement. Instead, the first priority of schools would be to ensure that by the time young people leave, they are able to relate well to others, are prepared to take responsibility, and ready to contribute to the wellbeing of their family and community. Alternative goals based on relational thinking in the criminal justice system would have far-reaching consequences. Instead of a system aimed simply at retribution, or at rehabilitating offenders, the primary goal would be to reconcile relationships between offenders and the victims of their crimes, permitting them to be restored into responsible membership of their community (Weaver 2016). Relational businesses would no longer have the primary goal of maximising shareholder value at whatever cost to the other stakeholders. Recognising that there is more to sustainability than shortterm profits, a relational company would seek to maximise relational wellbeing among all the stakeholders.

\section{Summary: a new way of thinking and making society}

The world system based of the financialisation not only of economy but, we may well say, of all social relations, experiences a chronic crisis and has to be reconverted. But how? In this chapter, I have argued that we do not have to resort to an abstract societal "model" but rather to facilitate ways of life (forms of a modus vivendi) which can empower the practices of a civil society that is not subordinate to the compromise between state and market.

It is possible to apply to this new civil society a notion of "reconversion" by analogy with what happened to market reconversion, when we shifted from an economy based on large industrial concerns to the information and knowledge economy. It can be defined as a reconversion of civil society if we think of it as a bottom-up promotion of networks of social relations that do not meet the criteria of monetary equivalence and/or the functional imperatives imposed by state laws, but respond to the need to create relational goods. The reconfiguration of civil society according to this scenario will also redefine the ways of being of the state and the market. 


\section{Shedding light on society through a relational (not relationist) gaze}

\section{Which sociological look do we need to see the relational society?}

In this chapter, I intend to clarify what it means to have a "relational gaze," without which we cannot see the nascent relational society beyond modernity. Such a society can only exist if social interactions and transactions manage to build structures that are capable, in turn, of sustaining reflexively managed relational flows.

For example, if we want to understand the evolution of capitalism, we must ask ourselves how the concept and reality of what we call "capital" changes. Simplifying, we have three ideal-typical ways of observing the phenomenon: the objectifying gaze (which applies positivistic knowledge), the subjectivising gaze (according to which knowledge is observer-dependent), and the relational gaze. Whoever uses the former sees capital as the accumulation of economic power of a social class (or elite or lobby or other) over a mass of exploited people, with the processes of inequality that it entails. Who uses the second, sees capital as an ability to anticipate ("discount") the future, creating subjective expectations that produce a reality from which to profit by using the medium of money. Both these ways of conceiving capital have their own validity. But they remain inside modernity. I am interested in seeing if and how social capital changes as a kind of social relation - and social structure - that generates another type of society. For this purpose, another kind of gaze is needed, which I call relational.

\section{The issue of the scientific gaze}

The scientific gaze tries to render the observer's work "rigorous." Exactly what the term "rigorous" means is somewhat debatable. For classical scientists, it means that the work in question is appropriate to the observed object, and as such is controlled, replicable and devoid of any distortion; for those sciences based on constructivism, on the other hand, such terms lose their value since a gaze creates, even imagines, its object. In any case, each science has more than one way of looking at reality, depending on 
how it constructs its relationship with reality. Such a relationship is a gaze, called "knowledge." Precisely because it is a relationship, a gaze represents a combination of the four components of any relationship, that is the value attributed to the gaze, its situational goal or intention, its means, and its norms of use.

What happens in the case of the scientific gaze is similar to what happens when a light is switched on in a room. What is seen depends on various factors: on what the person switching on the light wishes to see, on the observer's visual capacity, and on the characteristics of the device projecting the rays of light in terms of the rays' colour, their intensity, and their capacity to penetrate the observed objects. Each device possesses different qualities and powers, and each observer is interested in seeing different things.

Important questions of social ontology, epistemology, and social practices emerge here.

As regards social ontology, the observer may adopt a realistic stance, which presupposes the existence of a reality beyond the observer, together with the possibility of distinguishing the observer from the observed (Porpora 2018a). Alternatively, the observer may adopt a constructivist position whereby, in the words of Luhmann, reality is the observation itself (Rasch 2012). This approach leads towards deconstructionism, where the gaze is an expression of nothingness, of the void, of insoluble paradoxes, and thus a sort of optical illusion. On the other hand, "critical realism claims that it is possible to gain knowledge of actually existing structures and generative mechanisms, albeit not in terms of a mirror image - in this respect Rorty is correct in his criticism of the naive realists - but certainly in terms of theories, which are more or less truthlike" (Danermark et al. 2002: 10).

Critical realism, constructionism, and deconstructionism are ontological positions implying very different epistemologies and practices.

The transition from the 20th to the 21st century was characterised by a boom in constructionist and pragmatist approaches that completely subverted the realistic perspective of the sciences. Tired of, and disinterested in, an understanding of the world based on structures and dynamics, these new approaches proposed a vision that constructs the world around selected values and self-referential operations, thus deontologising observed reality. Constructionism is sometimes a silent ontology, and at other times a flat ontology: "[c]onstructionism is ontologically mute. Whatever is, simply is. There is no foundational description to be made about an 'out there' as opposed to an 'in here', about experience or material. Once we attempt to articulate 'what there is', however, we enter the world of discourse [...] The adequacy of any word or arrangement of words to 'capture reality as it is' is a matter of local convention" (Gergen 1994: 72-73).

According to critical realism, we need to distinguish three domains in the world (Bhaskar 2008): the observable domain of social phenomena (the "empirical" domain), where events are witnessed; a further domain existing beyond human experience, where the causal structures resulting in 
observable events interact (the "actual" domain); and a third, empirically undetectable domain where the potential, but non-actualised, underlying causal structures of objects are located (the "real" domain). This scheme of things represents a "deep ontology", unlike the flat, monovalent ontology offered by empiricism.

The gaze of a person adopting the social ontological perspective of critical realism, not only tells us what is "real" insofar as we see it in its empirical manifestations and in its ongoing causes, but also reveals the potential of that which exists within the depths of reality (reality as potential). It avoids ideological, abstract utopias, but offers a "concrete utopia" instead.

Without doubt there are innumerable styles of gaze, just as there are innumerable ways of relating to the world. These include narrative, discursive, highly imaginative, dramatic, scientific and futuristic styles, among others. Nevertheless, ultimately, we always end up asking ourselves what exactly it is that our gaze gathers.

Kenneth Gergen's "turbulent ontology" suggests that diverse gazes can be seen as the products of different confluences of relating between people's different activities and experiences (Shotter 2012: 10-12). While these confluences of relating undoubtedly play an important role, this does not mean that in a gaze reality loses its boundaries, its stratified structure, that is the layers constituting that reality. Following on from Kenneth Gergen (2009), what is meant by the idea that an observer's view of relations, such as work or the family, reveals ceaseless, unrepeatable forms of life consisting of intermingling movements? If Gergen's perspective, according to which "we live our lives embedded in the turbulent flow of a number of intermingling activities" (Gergen 1973) is taken to the extreme, the result is a gaze that is incapable of identifying defined structures and processes, because that gaze only perceives conflations between elements and layers of reality.

Critical realism does not deny that the observer plays an essential part in perceiving and processing what is observed. However, it distinguishes between strong constructivism and weak constructionism and only accepts the latter subject to certain conditions. As Bhaskar and Danermark (2006: 283-284) point out, "To weak constructionism, which involves the idea that there is a necessarily interpreted element in the construction of any theoretical understanding and any social object, a critical realist has no objection. However, if [constructionism] is taken to imply that the phenomenon investigated is just a theoretical interpretation or cognitive construction, or that a social phenomenon such as some specific form of disability exists only as an idea or belief, then it is clearly false."

A clear distinction needs to be made between the relational gaze and the relationist gaze. Many of those who have declared their adoption of a relational gaze, in truth, have been relationists insofar as what they adopt is a pragmatist approach that distorts the meaning of "relational" by transforming it into a mere interactive or transactional process. Emirbayer (1997), in his Manifesto for a relational sociology, never defines what he means by 
"relation" and "relational sociology", but instead talks of a "transactional sociology." Many authors followed Emirbayer's example by further radicalising his constructionist pragmatism. Considerable confusion ensued, to the point where Dépelteau and Powell (2013) came to the conclusion that relational sociology is nothing more than a play on words (Powell and Dépelteau 2013).

If one accepts these authors' approach, the relational gaze becomes a completely contingent, more or less random, turbulent transaction, with reality remaining inaccessible. The relationship between the observer and the observed risks being turned on its head: rather than being conceived as the observer's questioning of an observed or experienced reality, it becomes the exploration of what that reality could be in a purely contingent world. As Luhmann (2002) would say, that gaze is not so much concerned with understanding reality as it is, as with what "could be otherwise" (contingency is, quite simply, the fact that things could be otherwise than they are). These authors' gaze, even when understood as a relationship, is driven by the desire to transform human beings and society. As Gergen says, "As I will propose, when the logics of reflective pragmatism are fully extended, we enter a new territory of understanding, one in which the vision of research is radically altered. We replace the captivating gaze on the world as it is with value-based explorations into what it could be. This conception of a future forming orientation to research opens the way to new aims, practices, and reflections" (Gergen 2015a: 287, italics mine).

The idea that the researcher's gaze has to change the world rather than merely reflecting it does not seem to be perfectly consistent with Gergen's professed Buddhist ontology. On the one hand, he rejects Western traditions - both the Christian religious tradition and the secular Enlightenment worldview based on reason - and proposes a pantheistic, humanitarian, pacifist view of the world. On the other hand, however, he claims that "human nature is a cultural construction" that can and must be changed with a view to building a new "relational humanism" (Gergen 2015b: 151).

Given these characteristics, it is easy to see why Gergen's humanism, like that of all radical constructivists, leaves ample room for some version or other of post-humanism or trans-humanism. It ends up negating the boundaries between the human and the non-human, and as a consequence, the distinctive character of the human gaze is lost. So, is there no difference between the gaze as a human relationship, and the gaze as an actor-network relationship? Perhaps we need to look a little closer at what we mean when we say that a gaze is a relationship; in other words, we need to further examine what we mean by the term "relational."

\section{The relational gaze}

Adopting a relational gaze means first and foremost, observing social reality as a product of relational processes that are responsible for generating 
social structures and for reproducing or modifying them over the course of time. This viewpoint does not reduce relationships to structures, just as it does not reduce structures to relationships. Relations work within a structural context, regardless of how fluid and dynamic they may be, and this context is relationally constituted. Structures and relations forge a multilayered social world. Within a stratified social ontology, the assumption is that social relations and structures are co-principles of social reality, without there being a conflation of the two.

In my view, it is certainly correct to say that social relations are "flows," in the sense that they consist of reciprocal actions and transactions generating processes endowed with a certain fluidity (degrees of contingency). Social structures are not fixed as material buildings. Social relations always have some dynamism. However, as Bateson (1972: 83) argues, "The river shapes the banks and the banks guide the river. Similarly, the ethos models the cultural structure and is guided by it." To keep the metaphor, processes and transactions (the waters) flow within a context in which they themselves are conditioned by structures (the river), which they themselves forge in turn.

In general, a social structure changes through a morphogenetic process that starts from an initial structure at time T1, and through an interactive phase occurring between time T2 and time T3, generates an elaborated structure at time T4 (Archer 1995) (see Figure 4.1). Dépelteau, as most relationist sociologists, is right in analysing what happens during the T2-T3 phase, when relations show their interactional moment, but he fails to see the structure emerging at the end of each cycle of change. He sees only "continuity of change": "We never get involved twice in the same social process (in the same 'couple,' 'family,' 'classroom,' etc.)" (Dépelteau 2018: 503). He does not see the interplay between interactions and structures for two reasons: firstly, because his ontology is flat ("single-level ontology"), not stratified, and assimilates relations-as-interactions to relations-as-structures; secondly, because he does not distinguish between the temporal phases. It is important to understand that relationships are the object of sociology both as interactions-transactions (relationship as a process), and as emerging effects that possess a structure (relationship as a structure).

What I want to underline are the differences that exist between different orders of social relationality, that is, between the processual (interactional and transactional) order and the relational structural order, the latter being the outcome of processes that formulate structures (see Figure 3.1). I believe that this is the framework with which points of convergence between relational and processual-transactional sociology can and ought to be found (Vandenberghe 2016).

The social interaction of Ego and Alter always occurs within a specific social context constituted by a conditioning structural network. Of course, as White (1992) states, dyadic interactions are already embedded in a larger 


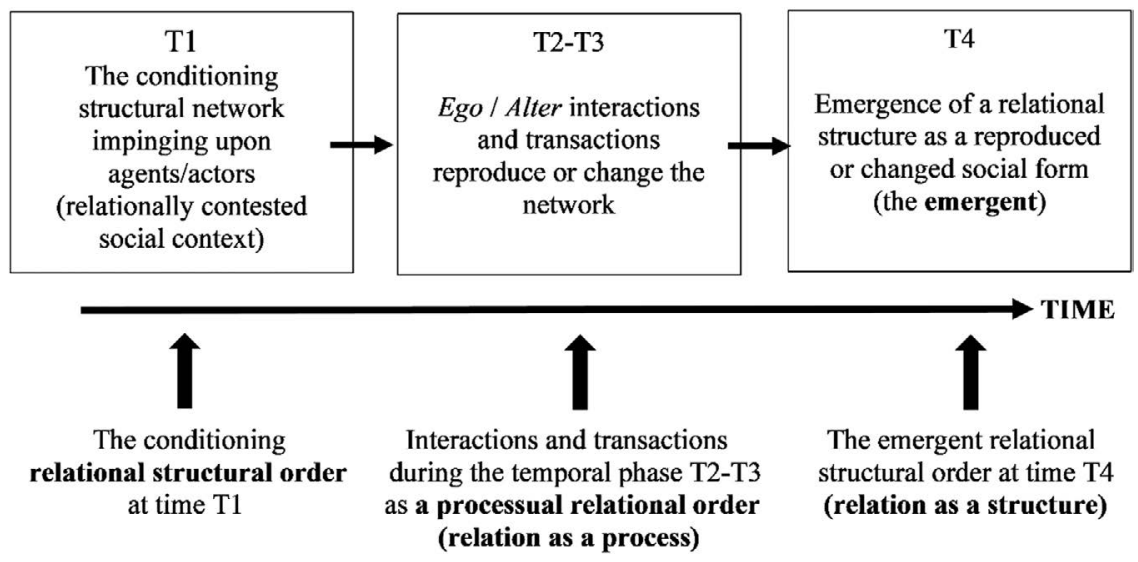

Figure 3.1 Different orders of social relationality: processual (interactional/ transactional) and structural

Source: Author.

network that includes the netdoms of Ego and Alter. ${ }^{1}$ Here I limit myself to exemplifying the two-way relationship for sake of simplicity.

If we look at the interactions and transactions within the temporal interval T2-T3, we observe the relational order of reality as a process (in which the structural network is questioned and can be reproduced or changed). However, when interactional networks stabilise, albeit temporarily, after a certain number of T1-T4 cycles, a new context is somehow generated. A new structural network emerges which, as a structural relational order of reality, will impact subsequent interactions and transactions (as in Figure 3.1).

The structures (rivers) are emergent phenomena constituted by flows (relations in the temporal phase of interactions and transactions) through the cycles of morphogenetic and morphostatic processes. They regenerate continuously after the water (the flow of relations) has gone. So, at time T1 of the morphostatic/morphogenetic process, we can observe a certain structure (the river), while at time $\mathrm{T} 4$, we see another structure emerging from phase T2-T3 of interactions, transactions, and exchanges. What happened between $\mathrm{T} 1$ and $\mathrm{T} 4$ is certainly dynamic and fluid, but this does not mean that structures are purely fictitious or emerging in the sense of appearing but without having any stability and consistency. This happens in physical

1 White (1992) defines networks as social spaces with broader temporal relational extensions than any smaller abstraction of momentary dyads; domains are the lifeworlds constituting the phenomenological contents and horizons of those relational spaces; networks and domains - netdoms - merge in type of tie, delivering a set of stories and sense of temporality to the relationship. 
(quantum) reality as well as in social reality when we go from the micro level to the macro level through the meso level.

Supporting my argument are the studies by Jussi Lindgren and Jukka Liukkonen (2019) who have offered a new interpretation of quantum mechanics according to which reality does not depend on the person measuring it. These authors concluded that the correlation between a position and momentum, that is their relationship, is fixed. In other words, reality is an object that does not depend on the person who measures it.

Therefore, those who speak of a liquid society, such as Bauman, must ultimately recognise that behind and beyond liquidity there are precise structures of inequality and discrimination.

I would agree with relationist sociology according to which the relational order of society has a transactional character when we look at it in a certain temporal phase (the phase T2-T3, between time T1 and time T4 of the morphostatic/morphogenetic process). Surely, one cannot enter the same water at different times, because the flow is unceasing. However, one can enter the same river, where the term "same" means the ipse (the same substantive entity), and not the idem (with the same characteristics as before), according to the distinction made by Ricoeur (1990).

In sociology, the processual perspective cannot be intended as a "metaphysics of becoming" based upon a radically flat ontology, because otherwise we could not account for the fact that social relations as transactions (the water) generate structures (rivers) that qualify, and cause the relational configuration of, the social fabric. This is the relational gaze.

Quantum physics states that the world is made up of relations that appear now as waves and now as corpuscles. For its part, relational sociology holds that the social world is made up of relations that appear now as processes (flows, wave equivalents) and now as structures (corpuscle equivalents). The problem that remains to be solved is to understand, in physics, how waves become corpuscles and, in sociology, how processes become structures, that is how we move from micro to macro. As Figure 3.1 shows, the relational thinking I propose constitutes the bridge between the two theories: in physics, between classical mechanics and quantum mechanics, and, in sociology, between the structuralism of classical sociology and the processualism of relationist sociology, ${ }^{2}$ as it does an operation that they do not see, that is it connects the micro to the macro through the meso level, which is the level at which the elements of interactions and transactions combine to give rise to an emergent. As I wrote with Archer (Donati and Archer 2015: 13),

2 As is known, quantum physics has been heavily influenced by the Upanishads: Subhash Kak (2008). The Wishing Tree: Presence and Promise of India. Delhi: Munshirm Manoharlal; Carlo Rovelli. Helgoland. Milano: Adelphi, 2020. The same can be said for the influence of Oriental thought (Veda, Upanishads) on relationist sociologists with respect to the conflation that they make between mind and reality. On the issue of emergence, see Porpora (2018b). 
"We defend an emergentist conception of relationality, which generates real and causally efficacious - but not 'substantialist' - emergent properties that unite this approach with Critical Realism. (Henceforth, those who reject emergence will be referred to as Relationists.)."

\section{The difference between observing and gazing}

Observing is an operation of indication. Indication does not become knowledge if it does not go through the process of recognising what one intends to know. And recognition is a relational and reflexive process.

The sociological gaze is not simply observing something/someone from a distance; it is not an aseptic, emotionless operation devoid of momentum (as in Luhmann, for example), but is an "involving operation," that is an operation in which the observer is involved in what is being observed. For this reason, it possesses a theoretical value (the search for the truth) and a practical value (insofar as it is a critical action in regard to the world), which is quite unlike that of impersonal observation.

Hence, the idea that a gaze, unlike mere seeing, is not something that can be offered by a third person, but requires the viewpoint of the first and second person too; indeed, it involves the perspective of the interplay between the three perspectives. The problem is how these three perspectives are to be combined.

What is missing is a sociological outlook achieved through a complex relationality that is not limited to one of them. In other words, we need to understand the complexity of the "relational gaze."

This is not taking the "middle ground" between objectivism and subjectivism, since it presupposes "another" viewpoint different from the objectivity-subjectivity dualism. The relational gaze is based on a social ontology, an epistemology, and social practices that are all relational because they have abandoned both holism and individualism. This gaze applies to all realities, but is particularly significant in the case of sociology where the object consists of social relations, their genesis and morphogenesis, their processual dynamics, and their established structures.

For example, when we encounter a group of people, it is normal for us to wonder who they are, that is whether they are members of a professional team, an informal group of friends, the members of a club or association, simply a group of tourists on holiday, and so on. In attempting to establish the nature of the group, we inevitably identify the group in terms of the relations between the group's members. The identity of the group does not correspond to individual characteristics, but to relational characteristics. In practice, if we wish to answer the question "who are they?", we have to carry out three operations: (i) acknowledge the group of people we see (the empirical phenomenon); (ii) ask ourselves what structures and mechanisms are at work in the interactions and relations between the group's members (the domain of the actual, i.e. what it is that makes the relationship between 
these individuals social); (iii) and ask ourselves which potential structures and mechanisms are not revealed (i.e. are not empirically visible), but could underlie the dynamics of that group (the domain of the real as a latent structure).

We can adopt this gaze in regard to all social phenomena, such as work, the family, health, well-being, solidarity or conflict, domestic life, friendship, and trust. Take the case of work, for example. The three phases of a person's gaze when observing this phenomenon consist in the observer focusing on, (i) work as the performance of services by one or more persons (empirical); (ii) which social structures and mechanisms give rise to said services, and what and/or who renders them such (actual); (iii) the potential capacity for change possessed by those structures and mechanisms giving rise to the activities observed (real). This is how the relational gaze encourages us to transcend the taken for granted.

A gaze becomes relational the moment that it focuses on the relationship between the observer and the observed and identifies the terms of the relationship on the basis of its causal properties and characteristics, rather than on the mere appearance of interactive processes. In fact, interactional pragmatism goes no further than the first two operations ( $a$ and $b$ ), thus failing to reflect on the latent structure of the relationship between agents/actors and on the potential generative mechanisms of that relationship.

A gaze thus becomes truly relational when it employs meta-reflexivity in the form of relational reflexivity. Relational reflexivity is defined here as the reflexivity that an agent exercises not on him/herself in relation to the context (as inner conversation), or on what the Other thinks or does, but on the relationship with the Other. It is therefore an act that operates as a relationship-steering mechanism, as opposed to automatic or non-relational social mechanisms (Donati 2015b). Let us now examine what this means exactly.

\section{What do we mean by "relational"?}

The concept of "relation" is widely considered problematic, in which it is polysemic and is used at various levels of reality, with diverse meanings, and often in a confusing, indiscriminate manner.

I propose to distinguish between five different meanings of the term "relation," based on the ontological status of the term and on the setting in which the relation is observed.

\section{Relations in the logical sense}

This is the primitive meaning proposed by Aristotle as a logical category. Saying that there is a relation between something (or someone) and something (or someone) else implies that a certain distance or proximity (to pros ti) exists between the two. The "structure" of the relation is that of a logical entity (of the observer's thought) of a "spatial" character, which does 
not have any consistence in itself, but depends on substances, that is on the terms that are placed in relation to one another. A relation as such is an accidental property that needs substances (the terms of the relation) in order to exist. This classical idea of relation is totally inadequate when trying to understand the meaning and weight of the modern concept of relation as the emergent effect of the mutual action of two or more agents.

\section{Physical relations with the natural world (physical setting)}

These are physical relations impacting the subject. For example, a child touches something very hot, this causes the child pain, and he/she pulls his/ her hand away and becomes aware of the fact that very hot things should not be touched; we notice a beautiful sunset and we stop to contemplate it; if a storm strikes accompanied by thunder and lightning, our reaction is to seek shelter. A relation dictated by the physical senses, which signals pleasure or pain, is a pre-social relation; but this relation is then processed in the mind through cultural mediation, meaning that this processing is conditioned by cultural habits and manners acquired from previous interactions. For example, when looking at a beautiful sunset, a person may experience feelings that translate into previously acquired images which the observer may reflect on through the mediation of previous experiences. The view of a tornado arriving triggers relations mediated by what the observer has discovered in the past. In any case, the relationship with the natural world is not a reciprocal action between subjects. It is a subject-object relationship. The relational gaze here consists in the way in which the subject processes the response to physical stimuli on the basis of his/her own acquired sociocultural experience.

\section{Relations with the practical world}

In the practical world, a relationship is a position adopted in regard to things, situations, and contexts insofar as they are human artefacts or the products of society. As Archer (2010: 294) writes, "in the Practical Order, tacit skills are emergent from the affordances and resistances presented by objects and the assimilation of and accommodation to them on the part of the subject. Activities such as competently playing tennis, a musical instrument, touch typing, or driving all depend upon "catching on" and, at more advanced levels (such as improvising at jazz or manoeuvring an articulated lorry), upon acquiring a real "feel for the game." From my point of view, in this order of reality, the use of objects constitutes a social practice that implies a specific relationality (e.g. when we prepare a family lunch, go to the gym, drive a car, or interact with a robot). What is of fundamental importance if we are to understand the meaning of these relations is that their referent is an "object" which encapsulates the representation of actions previously performed by human beings, and therefore, it incorporates a relational message so to speak. The relational gaze upon these 
"object relationships" requires a sort of relational "dialogue" with objects and not just a physical relationship as in the previous world. For example, if somebody receives notification of a message on Facebook, that person's mind will respond to the message on the basis of the relationship that the message implies. In this world, specific mention should be made of relations with smart AI and robots, since they are not inert objects. A relationship with objects possessing their own "autonomy," such as sophisticated robots, raises specific problems in those cases where the agent treats such robots as if they were "people." In these cases, it is necessary to distinguish between human and non-human relationships.

\section{Relations with the social world, that is with human beings and groups of human beings}

In this world, a relationship is both an orientation or symbolic reference having a reciprocal meaning (as defined by Weber) and a bond between people and their social roles underlying morality and social integration (according to the sociology of Durkheim). It may initially consist of a meaningful orientation that generates a bond or a bond giving rise to an inclination; in either case, the two components (symbolic reference and social binding) are connected and interwoven. I have called these two elements refero (symbolic reference) and religo (tie), and I would argue that their combination produces an emergent effect, that is a social relationship as a social structure with its own characteristics and causal powers. Here, the nature of a relational gaze is that of an effect deriving from the contributions of the related subjects, who generate an emergent.

\section{Latent relations with a reality that transcends the empirical and the actualised}

Relations with realities that are hidden from view because devoid of all materiality - such as religious or supernatural realities - are what we may call latent; however, they are no less real as a result of their latency. These latent relations possess their own potential. They connect us with a world that we cannot experience through the physical senses, but that includes important realities (called "values," "ultimate concerns," "living symbols"), which we cannot measure using quantitative methods, but which are key to the forging of the subjects' identities and the structure of such relations. These values include trust, peace, and fraternity, which are of an invisible, intangible nature. The relational gaze on these realities goes beyond any established cultural system and sociocultural interaction, because cultural meanings are reinterpreted in order to reach a meta-reality.

I have distinguished between these five types of meaning of the term "relation" in order to avoid any generic definition and any confusion between the different layers of reality (stratified social ontology). 
Let us take as an example the statement "things speak to us," as if things non-human objects - were capable of relating to us humans. The relationship between us and the objects involved in this statement can be interpreted in different ways, which are precisely different layers of reality. The relationship can be seen as a logical relationship, as a physical relationship, and as a practical relationship. If the object is natural (a stone, a cat, a flower), it seems to me difficult that the relationship can have the characteristics of the inter-human or transcendental one. The case in which the object is a human artefact is different, because in the artefact (like a work of art or a robot), the relational gaze can glimpse the intentions of those who produced it to establish a certain relationship of human value.

Of course, when we see great works of art or a beautiful sunset, this triggers feelings and emotions in us, and these stimulate thoughts which have a relational character for us, but this dialogue is entirely processed within our Self (Scheve (von) 2018). Objects offer us perceptive stimuli, memories, signs, and symbols which we ourselves give meaning to, and which we mentally "converse" with/reflect on in order to relate to them. If we want to understand the contribution to the relationship made by the observer and by the observed respectively, a reflexive relational gaze is needed.

Bruno Latour's Actor Network Theory (ANT) currently lends support to the argument that the human/android distinction will gradually cease to exist, insofar as it sees the network of relations between humans and non-humans as a subject-agent acting on its own behalf (the actant). What kind of relational gaze can there be if, as Latour argues, the distinction between humans and non-humans gradually ceases to exist?

As Whittle and Spicer (2008: 611) claim, the "Actor Network Theory, otherwise known as the sociology of translation, rejects the idea that 'social relations' are independent of the material and natural world." Latour's view, like that of all relationists who eliminate the aforesaid distinctions, leads to one losing sight of the unique nature of social relations. If we lose the specificity of the different relationships and their own contribution, we can no longer see the responsibilities of the network's various agents. As Graham Harman (2009) has pointed out in his object-oriented philosophy, the relationist metaphysics of Latour cannot properly accommodate the capacity of objects to cause or mediate surprises for other objects.

I wonder what kind of gaze and what type of relations can be attributed to the actant. What relations can human beings have with the actant? Latour suggests that we adopt a "hybrid gaze" that eliminates the subject-object distinction (Blok and Elgaard 2011). The social (i.e. the relation) takes the form of indistinct interactions, and eventually vanishes, the moment the human subject disappears. The relational gaze of sociology no longer possesses human characteristics, becoming as it does a hybrid thing.

At this point, the problem is that of understanding what is meant by a sociological gaze that has been rendered so "hybrid" by technological 
progress that human beings now question their own identities and capacities. In order to understand such changes, a truly relational gaze is required.

\section{The sociological gaze needs a relational reflexivity}

If the sociological gaze is a way of reflecting on what surrounds us, bearing in mind our own inner reflections, then we must understand what it means to be reflexive "in relation" to a context. Let us take the definition of reflexivity given by Archer (2007: 4): "reflexivity is the regular exercise of the mental ability, shared by all normal people, to consider themselves in relation to their (social) contexts and vice versa." What does "in relation to" mean here?

If we accept this (mental) definition of reflexivity, then it is difficult to understand what role the relationship plays as an entity distinct from the self that thinks within itself. The concept of relationship runs the risk of playing a purely logical role. The Ego's gaze seems to be sociologically relational because it takes into consideration what comes from other people, together with the opportunities and constraints offered by the situation, and reacts reflexively from its own viewpoint. "Reflexively" here means that the Ego pursues what it believes to be a good life through an internal conversation that firstly defines and dovetails the Ego's own concerns (internal goods), then develops practical courses of action (individual projects as micropolitics), and finally establishes sustainable practices (modus vivendi) that can satisfy the Ego itself.

What this definition of reflexivity fails to account for is the role that the social relationship plays in the reflexive process, as an emergent reality with its own causal powers and properties. In my view, the relational gaze requires relational reflexivity because the correctness and reliability of the cognitive process is guaranteed by rendering the relationship with the object of investigation reflexive ${ }^{3}$ and not by the correspondence (or symmetry) between the mind of the knowing subject and that mind's object.

For example, one may wonder why parents who themselves are good people have children who are socially deviant, and, vice versa, how it is that individually deviant parents manage to have honest, successful children. Empirical research shows that such outcomes can be ascribed to the fact that children are socialised not so much by their individual parents, but rather by the relationship that their parents have with one another. The socialisation of children is a result of the relationship between parents, ceteris paribus. Different outcomes depend on how relational reflexivity works within the family. While in the first case, well-meaning/good

3 "Reflexive" is not the same as "reflective." Reflexivity is an act of relating to an object by an acting subject; therefore, it implies an agency. Reflectivity is an automatic reaction effect, or a state of being reflected, as happens when light is reflected from the mirror. 
parents may have deviant children because they, the parents, fail to be reflexive in regard to their mutual relationship, in the second case, deviant parents (such as drug addicts and criminals) may have successful children because, albeit unconsciously, they actually exercise a positive relational reflexivity between themselves. On a practical level, by reflexively modifying the relational dyadic or group network, deviance can be prevented (Weaver 2016).

What gaze do individuals adopt on the basis of their inner reflexivity? Let us examine the ideal types of reflexive mode identified by Archer (2003, 2007). The gaze of communicative reflexivity is dependent on the outside world ("significant others") and is mediated by family attachments because the Self is incapable of separating itself from such attachments. The gaze of autonomous reflexivity is self-centred or self-referential. The gaze of meta-reflexivity is prompted by the individual's dissatisfaction with the existing situation and by criticism of what that individual's action has achieved. The gaze of fractured and impeded reflexivity is wholly disoriented and leads to inaction, since such people find it very difficult to make decisions, to establish courses of action to be consistently pursued, and above all to engage in anything more than survivalist day-to-day planning; their internal conversations cannot lead to purposeful action, but tend to exacerbate personal distress and disorientation resulting in an inability to think, deliberate, and act with conviction.

In developing her original analysis of these different types of personal reflexivity, Archer examines how social structure (continuous or discontinuous, etc.) conditions individuals (without determining their courses of action however); yet there is no in-depth focus on the role played by the social relationship that mediates between the structure and the specific modes of reflexivity characterising different people. If one believes that the mediating social relationship plays a secondary role derived from the inner conversation, then we are led to Dépelteau's (2013: 818) argument that we need to abandon the concept of structure in order to give priority to pure interactions and transactions.

I don't think this is Archers' intention, but in any case I wish to clarify my point of view. I argue that, on the one hand, social structures influence personal reflexivity by acting not directly on the individual's mind, but through the social relations that give shape to personal identity and are acted upon by relational reflexivity. On the other hand, only by focusing on how relational reflexivity is implemented can we ascertain the influence of social structures on the acting subjects (and understand the structure of social relations).

According to my relational sociology, the gaze becomes relational if the investigator observes the contextual relations as such, together with their independent dynamics, in order to perceive how relations - as real entities existing outside of the observer - modify the mental activities (reflexivity) of the individuals concerned and their re-actions. This is what I mean when I say that a relational gaze is achieved through a relational reflexivity (Donati 
2011b). We need to understand the limits of a vision of reflexivity consisting of an individual's internal conversation, as well as the vision of reflexivity as determined by structures or social system, if we are to see the relational reflexivity that is necessary in order to achieve a relational gaze.

I believe that the concept of social relation employed by Archer is not very far from the definition of social relation proposed by Max Weber:

The term 'social relationship' will be used to denote the behaviour of a plurality of actors insofar as, in its meaningful content, the action of each takes account of that of the others and is oriented in these terms. The social relationship thus consists entirely and exclusively in the existence of a probability that there will be a meaningful course of social action - irrespective, for the time being, of the basis for this probability.

(Weber 2013: 26-27, emphasis mine)

In other words, the social relationship is traced back to the actions of individual agents, since the action of each actor, to be meaningful, takes account of the actions of the other actors and is oriented in such terms. The relationship is not an entity that exists between them with its own reality.

Weber's definition is partial and insufficient for the purposes of a comprehensive sociological analysis. It fails to offer an in-depth view of social phenomena, since it only grasps the referential orientation of the relation (the refero), whilst ignoring its binding structure or structuration (the religo), and does not account for the emergent impact of their combination.

Weber does not mention the subject's acknowledgement of the worth of the relationship itself. The worth of the relationship is given by the individual subjects concerned and does not possess its own ontological status. Even when Archer talks about relational reflexivity, the internal evaluation of the relation or relationship is the one given by the person concerned; that is, it is how the agent sees, considers, and values that relationship subjectively.

If we accept the idea that a gaze, including a sociological gaze, is the product of the observer-agent's mind, then we risk adopting a radically constructionist position whereby the individual may take on any sociocultural identity he/she chooses. In fact, many current-day scholars, including certain critical realists, accept the constructionist approach in regard to gender roles, as if these roles were fully editable, and entirely equal to, or interchangeable with, any other social role. In this regard, Archer (2019a: 29) argues that the identity of a human being (i.e. human personhood) does not concern that person's bodily constitution, since it consists in the capacity to adopt a first-person perspective. But, if we assume that the body is irrelevant for the mind, it also becomes irrelevant to social relationships. Therefore, the latter become pure mental entities, and, with the advent of ICTs, they can be identified with internet connections.

Thus, one can understand Archer's (2019b) statement according to which relational reflexivity, which regards social interaction, "is not different in 
kind" from personal reflexivity. In this way, the relational gaze is dependent once again on the subjectivity of the agent and does not concern the relationship between the subject and the Other, while from my point of view, relational reflexivity is, on the contrary, of a different kind from personal reflexivity.

To understand relational reflexivity, one may ponder the question: "am I willing to be questioned by reality (or by the Other)?". The willingness to be questioned thus means changing one's reflexivity because of the relationship, that is because the relationship raised by the question changes the context itself so that a new relationality is required. What reality (or the Other) tells me may change my perspective, that is my manner of thinking in the first person. The Self becomes involved by what is not the Self. In other words, the Self must consider the existing relationship with reality and with the Other, while bearing in mind a point of view which is not its own.

A relational gaze - the relational perception of things - clearly means questioning reality on the basis of one's own interests; however, this operation is not confined to within the observer. The fact that the relational gaze adopts the viewpoint of the relationship means that it is the result of a combination of first-, second-, and third-person perspectives. One perspective does not suffice if one wishes to grasp the relational nature of reality. The relational gaze avails itself of all three perspectives. Of course, exactly how these three perspectives are utilised will depend on the narrative context.

For example, if John turns to a friend and says: "Why don't we go for a walk together on Sunday?," John's gaze is on his friend in the first-person. The "We-relation" (going for a walk together as a "We-relation") is only a possibility, a symbolic reference, and not a certainty, a binding tie. If, on the other hand, John is invited by a colleague to a party to be held the following Sunday, and in reply to the invite says, "Ah, next Sunday I'm going for a walk with my wife," then John is speaking in the first and second persons, since he is also speaking on behalf of his wife and including her in the We-relation of the Sunday walk, and replies to his colleague from the point of view of that relation. If John is telling his friends how he spent the previous Sunday, and says, "Did anyone see my wife and me together last Sunday?," he is adopting the perspective of a third person, because it is the viewpoint of an impersonal gaze, that is it refers to what happened in the third person.

The first-person perspective is clearly unavoidable, since the gaze is that of the observing subject. The second-person perspective entails dealing with the Other's concerns, which means adopting the view of a reality that goes beyond one's own interests, that is the relational good, which transcends and resolves the problem of the dual hermeneutics (Donati 2019). The third-person perspective renders the observation impersonal and, in some way, represents the implicit or explicit finalism of the relationship that the observer is observing from the point of view of the sociocultural context. I believe that there is no reason to negate the role played by the third-person perspective: this term can be interpreted as the reference to the objectivised 
"we-ness" of the relation, that is to the actualisation of the being together, which goes beyond both Ego and Alter, from the viewpoint of the impersonal sociocultural context. The third-person perspective expresses an impersonal gaze which is common currency in everyday conversation. However, it has to be combined with the first and second-person perspectives if it is not to reify and depersonalise the gaze. For example, the gaze of a person who wishes to see the "relational reality" of an institution (such as family, school, or company) as a social phenomenon that goes beyond individual, contextualised cases needs to adopt the third-person perspective. However, this gaze, if it is to provide any meaning in the lifeworld, must be interpreted also from the first- and second-person perspectives.

\section{Summary}

I have tried to argue that in social sciences, as in other sciences, the diverse ways of observing reality should not entail the adoption of a purely relativist, processual, or transactional point of view. There is another way - through the relational gaze - of seeing and appreciating reality in its "being," that is in its ontological essence, which is not fixed but which unfolds within the framework of the contingencies that arise. I have discussed the conditions under which the relational gaze may be distinguished from other gazes, such as objectivising and subjectivising gazes, or combinations of both, or from other types of gaze altogether. The relational gaze makes intelligent use of first-, second-, and third-person perspectives, without relying on just one of the three. In other words, it is capable of distinguishing between personal reflexivity (internal conversation), relational reflexivity (meta-reflexivity concerning the relations), and systemic reflectivity (the reflective mechanisms of relational networks), and recognises the role that each plays.

The relational gaze focuses on the relationship between the observer and the observed, by adopting the point of view of the relationship and rendering the relationship itself reflexive. It cannot be restricted to the viewpoint of the observer, whose mind constructs its chosen object of observation; neither, indeed, is it simply the viewpoint of an external world that is introjected in the observer's mind. It is not a mechanical operation, but neither is it pure interaction or transaction. More specifically, it cannot be defined merely as the "observation" of an autopoietic, self-referential system. The relational gaze is the triggering of a relationship that is responsive to sense perceptions and emotions, and thus to feelings, which constitute a commentary on its ultimate concerns; but it is certainly not passive. It is a complex activity triggered by a person's relational reflexivity.

Relational society is based on this kind of gaze, otherwise it does not exist. 


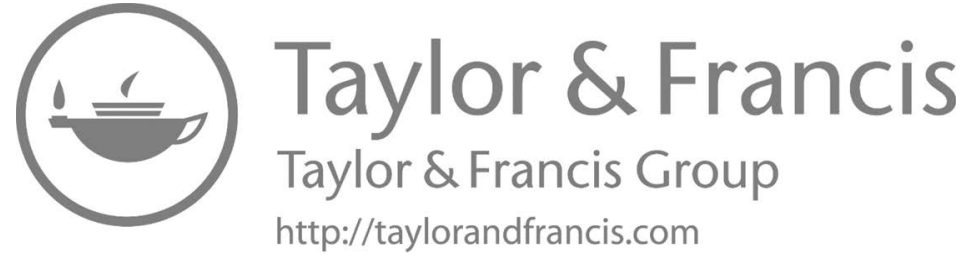




\section{Part II}

\section{Insights into the morphogenetic changes of social morality}




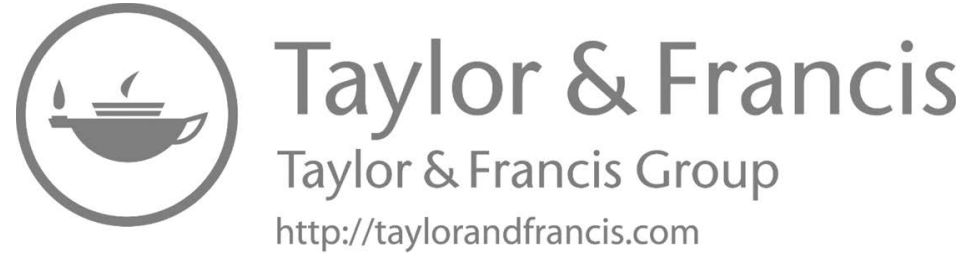




\section{Relational society as a morphogenetic configuration}

\section{How do we conceive of society?}

In the year 1991, talking about the so-called postmodern era, I wrote, “... what happens is understandable as social morphogenesis under conditions of high complexity." I suggested to rethink social theory and the representations of society by leaving aside the grand narratives of the past, and restarting from the very basic concept of social relation.

The social morphogenesis scheme appears to be particularly suited to give an account of how the objective and subjective factors, internal or external to a given society, combine and interact with one another so as to generate a society that is different from the preceding one.

The fundamental thesis of this chapter is that the morphogenesis of society comes about through social relations, which are the connectors that mediate between agency and social structure. The generative mechanism that feeds social morphogenesis resides in the dynamic of social relations networks. In order to observe this phenomenon, it is necessary to invoke a general theory of social relations.

What do I mean by "society"? What I call "society" is not a more or less orderly, more or less conflictual collection of agents/actors (whether individual or collective) that share an arena of action. The society of which I am speaking is made by individuals but is not made of individuals. Certainly, only individuals can activate it, but society is another thing with respect to what individuals are and carry in society, even if enhanced with smart technologies.

Every society lets itself be understood based on a fundamental criterion of a suprafunctional nature, which defines its operating modalities (i.e. how that society draws its guiding distinctions) and, thus, its qualities (how it conceives its difference as compared to other societies) and its powers (that for which it causes certain outcomes and not others). For example, if one

1 My translation from P. Donati. Teoria relazionale della società. Milan: FrancoAngeli, 1991, p. 11. 
chooses as basic criterion the kind of functional differentiation predominant in a society, then one arrives at distinguishing between segmentary, stratified, and functional societies, as Luhmann does. If one argues that a new society is arising different from the archaic, pre-modern, and modern ones, one needs to identify the basic criterion on which such a new society is founded in order to qualify it properly (Donati 2011a: 203-207).

All of the classical sociology adopted a particular criterion, which in general was dualistic or binary. For example, it contrasted pre-capitalist society and capitalist society (K. Marx), society with mechanical solidarity and with organic solidarity (E. Durkheim), communitarian relations and associative relations (M. Weber), Gemeinschaft and Gesellschaft (F. Tonnies), societies based on concentric circles and societies based on intersecting circles (G. Simmel), and so on. Many contemporary authors also carry on using dualistic typologies. For example, Berger and Luckmann (1995) propose the distinction between "integrated" societies and societies "exposed to crisis."

All these theories typically share a dualistic view regarding the process that leads from one type of society to another type. For relational sociology, one society distinguishes itself from others by the way in which its relations are qualified and by the way in which the networks of relations that define it are configured. To speak of the morphogenesis of a society thus means speaking of transformations of the social relations that make that society in as much as they "exceed" initial structures.

From the standpoint of the relational paradigm, society is the product of associative and dissociative relations that arise in response to conditioning societal structures and continuously alter them. It is a matter of understanding how the structural dynamic of relations creates a society that is different from others due to the fact that its relational generative dynamic is different. The social morphogenesis scheme seeks to fill this gap. It does this with the support of a theory of reflexivity that makes it possible to analyse differing forms of society on the basis of differing forms of reflexivity that each society promotes and enables. In any case, it is about different types of relationality that we are speaking.

In other words, "making society" implies that the social relations among participants are conceived and lived as a modus vivendi, that is, as a way of conducting one's existence with others in a social sphere that is not differentiated according to functions, but according to relations, instead: this is to say that it is distinguished by the sui generis qualities and powers that constitute it. Such relational modalities confer a specific configuration upon every "society."

A society is never perfectly integrated and conflict-free. It usually has problems with both social and system integration. Belonging to a "we" does not at all mean that internal diversities are lacking or negated. Internal differentiation (among agents, their cultures, their relations) is necessary for the existence of a society. Differently from the functionalist approach, 
relational sociology maintains that the guiding distinction of the aftermodern society is a form of relational differentiation that leads to configure society in a suprafunctional way.

\section{A theory of social relations and their structure}

When I think about social relations for what they are, it always occurs to me to compare them to air. What they have in common is the fact of being invisible to the naked eye and of being essential for human life. The analogy stops here. However, the comparison between what air is for the human body and what social relationality is for the identity of human persons can be instructive.

Air does not have its own molecular structure: it is simply a mixture of gases and of solid and liquid microscopic particles; its basic elements combine together, altering its composition while they take in or expel certain gases depending on conditions of pressure, temperature, etc. But it does not have a "form" in the same sense in which this concept is implied when talking of social relations. It might seem that the same thing happens for social relations, but this is not the case. The social form is not an aggregation or composition of elements but is instead the relational structure - active and modifiable - that exists and operates among its elements, which can be radically replaced by other elements. Air does not have this quality. What air can cause (good or bad weather) is of the order of probabilities. What social relations can cause is of the order of possibilities.

Many of the relations of everyday life are indeed highly contingent (random) in their composition of motivations, desires, feelings, aspirations, plans, rules, interests, means employed. Relations seem to fluctuate in a similar way to air in that they are composed of a mixture of elements, which are scarcely or not at all ordered, that continually alter the relations' quality. Air can be more or less hot or cold, dry or humid, still or windy, clean or dirty; it can acquire various types of scents and odours, and so on. The same thing may appear to happen to social relations: they can be more or less warm or cool, stable or unstable, and so on. Thus, it would seem that there is a decided resemblance between air and the social relation. Marx said as much in his famous pronouncement (stated in the Manifesto of the Communist Party of 1848), which was taken up by Berman (1982) in connection with the experience of modernity: "All that is solid melts into air." The solidity of the social relations of the past is transformed into air. But I wonder: is this how things really are?

My response is yes and no. On the one hand, relationships seem to become extremely fluid (think of the mixture of female and male characters in gender relationships). But, on the other hand, unlike air, social relationships tend to be structured according to particular forms (which I call "social molecules"). These forms consist of elements linked by a specific relationality that is exercised among them. Of course, the elements of the social relation 
are not of a chemical-physical order of reality. These elements belong to the order of action and interaction. So they include feelings, emotions, opinions, intentions, projects, material objects. All these elements enter exchanges and transactions from which an emerging effect is generated. This is how new structures are elaborated. Such structures are qualified by the kinds of qualities and powers pertaining to the social relations that make them up. In their turn, these qualities and powers of social relations decide the effects that the relations have on people and social formations.

Perhaps a virtual society is like air, but a concrete society has a tendency to structure itself. Indeed, whoever claims that our society is becoming "liquid" must then admit that, in fact, there exist social structures endowed with great stability and influence over individuals' lives (think of the structures of inequality).

Those who maintain the analogy between air and social relations evoke attractive images, but from an ontological and epistemological point of view, the analogy is very limited and ultimately misleading.

The ontological and epistemological presupposition of this perspective lies in the fact that relationality is the mode of existing of that which belongs to the social order.

I assume that "being in relation" is an expression which has three analytical meanings:

1 It says that, between two (or more) entities, there is a certain distance which, at the same time, distinguishes and connects them.

2 That such relation exists - that is it has a reality - in itself (from Latin "ex-sistere," which means "to be out having its own consistency" in respect to its generators) with its own qualities and causal powers.

3 That such a reality has its own modus essendi (the modality of "being" inherent to the relation), that is a structure, be it more stable or more volatile.

These three meanings are analytical, because - from an empirical view point - every relation contains all these aspects, which are closely linked to one another.

Let us turn now to the structure of the social relation and enter "inside" the social relation. If we define the social relation as an emergent effect of a reciprocal action between Ego and Alter in a social context, the relation can be seen from the subjective side (of Ego and Alter, respectively) or as "object" (objective reality) between the two.

Let me remind of the following three semantics of the social relation:

1 the semantics of the relation as refero, that is as a symbolic reference grounding in a motivating value intentionality;

2 the semantics of the relation as religo, that is as bond, structural connection made of norms and means; 
3 the generative semantics of the relation understood as an emergent from the combined provisions of refero and religo in a particular configuration (relational effect).

Many authors define the social relation in terms of a structure of interdependence between mutual expectations. Other scholars conceive of social relations as "transactions." Of course, these definitions touch upon relevant aspects, but have the great limit of not showing how the emerging structure is elaborated, because they do not enter into the relationship. They merely observe the effects produced by the individual actions of the agents.

In my view, the relation as effect of reciprocity (Wechselwirkung) takes on a form endowed with its own qualities and causal powers, which requires a reference to the specific social context in which the interactions between agents/actors take place. The context can define the social relation as a simple event (e.g. a person asks for a beer in any pub, pays, and leaves), or as a bond that was created through multiple reiterations over time (e.g. the relation between a patient and his/her personal doctor), or a bond that derives from ascriptive factors (e.g. the relation between parents and their children).

Let us take a synthetic look at the social relation's composition and form.

\section{Composition}

The social relation is composed of elements that come from Ego and Alter's actions but these acquire a different significance when they become elements proper to the instantiated relation since they enter into a new form (see the definition of the form on the next page). The elements in question are those that give meaning to action and are therefore very diverse: expectations, goals, means used, etc. with respect to certain individual affinities, utilities, needs, and values.

An interesting way to conceptualise the analytical elements of every single action is to group them into four categories: the goal or target $(\mathrm{T})$ pursued in a situation, the means used to reach the goal $(\mathrm{M})$, the norms $(\mathrm{N})$ that are followed in relating to one another the elements of the relation, and "latent cultural value" (C) that the relation incorporates.

The most difficult element to be grasped is the "latent cultural value." It is not an abstraction, but corresponds to the criterion used for evaluating the action's goal (T). It answers the question about "why" (in-order-to motives) an agent/actor performs an action and seeks a situational relation.

The relation as emergent can be analysed in its composition with reference to the above-mentioned four analytical elements, provided that we distinguish these elements as components of the single actions and as components of the elaborated structure. In other words, within the emerging structure, the action's elements take on a relevance different from those present in the agents/actors' single actions. The four orders of elements are not necessarily congruent with one another; on the contrary, their 


\section{Time1 - The social and cultural structure conditioning a given social relation $\mathbf{R}$ (e.g. constraints and cultural models that impinge upon the employer-employee social relation) -}

Time2 - Interactive network of agents / actors who can reproduce or modify the relation $\mathrm{R}$ by keeping the same elements and their combination or changing them - Time3

Temporal phases

\section{Time4 - Emergence of an elaborated R' that can reproduce or change the initial relation $\mathrm{R}$ (e.g. reproduced or changed employer-employee relations) within another conditioning structure -}

\section{$\rightarrow$ (a new cycle begins)}

Figure 4.1 The basic general scheme of the morphostatic/morphogenetic process by which social relations are reproduced or changed

Source: Author.

coherence is always problematic, at the levels both of single actions and of the social relation.

The combination in question, which has an autonomous relational structure with respect to the single actions that generate it, can be read as a black box. As a black box, it can be trivial or non-trivial, that is it can always generate the same output, or it can always generate different outputs. Of course, morphogenesis exists when the black box is not trivial.

\section{Form}

This is the relational structure that organises the elements coming from single actions and combines them in such a way as to impart to them a certain arrangement (relational effect) that has a causal power over the participants. It is a result of a morphostatic/morphogenetic process.

The analysis of this process (see Figure 4.1) starts at Timel by observing the relationship $\mathrm{R}$ in question as placed within a social and cultural structure that conditions it. For example, let think that we observe the employer-employee relationship as the focus of our research interest. Then, we get empirical information about the interactions between the actors/ agents in a temporal phase between Time2 and Time3. Empirically, we want to know how their actions reproduce or change the initial relationship $\mathrm{R}$ in the interactive network linking employers and employees (relational network analysis). This network is our black box, in which the agential elements 
combine so to reproduce or change the outcome, which is observed at Time4 as elaborated structure (relationship $\mathrm{R}^{\prime}$ ).

In general, in a social network, the mix is formed through the encountercollision (i.e. a certain combination) among the goals, means, norms, and values of single actions within the conditions at the network's boundaries.

In short, the social relation is the emergent effect of reciprocal actions reiterated over time among social actors occupying different positions in a societal configuration (system or social network). The relational analysis translates the network into a matrix $(\mathrm{i} \rightarrow \mathrm{j} / \mathrm{j} \rightarrow \mathrm{i})$ from which we infer that the relational effect is the result of Ego and Alter's contributions plus the contribution added by the relation as such (Tam 1989).

The reciprocal action (interaction), if stabilised for a certain period of time, causes an effect to emerge consisting in a structure of interdependence (or configuration of the relation, or its "figuration" as Elias 1978 would say), which can be reiterated or changed over time. The elaborated structure can be a (relational) good or a (relational) evil depending on the (positive or negative) influences that it has on the subjects/actors of the relation and depending on the consequences (outcomes) that it has on the broader network of interdependencies (social networks) in which it is embedded.

A fundamental warning: when I speak of the "target" or "goal" of a relation, this expression is to be understood as strictly correlated (if not equivalent) to the expression "guiding distinction" or "directive distinction" of a relation, which is the distinction used by the agents/actors in order to make a selection among a variety of possibilities. What the relation aims at (i.e. its end) is to keep the distinction that makes that relation specific in respect to other, different relations. For instance, if I visit the doctor, the goal of the relation is to get medical care; if I am to achieve this purpose, I must firmly distinguish between the therapeutic relation and other kinds of relations possible with the doctor. One must do a relational work aimed at distinguishing between this specific relation in respect to other relations.

In a human biological cell, antigens exist to help our body distinguish molecules as self or non-self. In a social form, antigens exist to help a peculiar social form distinguish social relations (molecules) as specific (self) or extraneous (non-self) to that form.

Let me explain this idea with the words of Viviana Zelizer:

In all economic action, I argue, people engage in the process of differentiating meaningful social relations. For each distinct category of social relations, people erect a boundary, mark the boundary by means of names and practices, establish a set of distinctive understandings that operate within that boundary, designate certain sorts of economic transactions as appropriate for the relation, bar other transactions as inappropriate, and adopt certain media for reckoning and facilitating economic transactions within the relation. I call that process relational work. 
Let us consider, for example, the doctor-patient relation. Both the agents/ actors have goals, means, norms, and values, which are in part convergent and in part divergent but, in any case, are understood subjectively in different ways. The relation that emerges is made of elements that come in part from the doctor and in part from the patient, and is an activity-dependent reality operating between the two. Although the relation is supposed to be of a therapeutic nature with a view to improving the patient's health, it is possible that the actions' elements do not lead to this type of relation but to some different types of relation. For example, in reiterating their relationship, patient and doctor happen to talk about their existential problems and the therapeutic relationship becomes a conversation over matters that have nothing to do with the health issues, or they develop a friendship relation, and one of them becomes member of a club attended by the other where the therapeutic relationship becomes informal instead of being restricted to the formality of the doctor's office.

Another example is the relation between partners in a couple. When Ego and Alter decide to form a couple, they try to adapt their respective situated goals, means, norms, and motivational values so as to create a relation from which each partner can receive what he/she expects. "Being in" this relation means pursuing some opportunities while accepting some constraints. Both the opportunities and the constraints are continuously renegotiated. The action of free giving, that is, the unconditional acceptance of the other's expectations by one or both parties, is possible but is an exception because normally the two partners try to find an arrangement that satisfies their personal needs. The difficulty with such an arrangement arises from the fact that the relation is a "third party" or tertium that mediates between Ego and Alter. It has its own reality, which is relevant not only for the partners but also for other actors/agents outside the couple who observe it, evaluate it, and have their own expectations of it. Whoever observes the couple attributes certain properties and powers to it that are different from the two partners' personal expectations.

We could say the same thing about the relation between employer and employee (which is formally spelt out in a contract that establishes the relation), or between teacher and student, and so on. The ways of combining the elements provided by Ego al Alter with their actions depend on the nature of the relation and of the social context (boundary conditions). If the setting is a family context, it will have a family form; if it is a medical office, a school, a business, a public assembly, etc., we will find different relational forms for each of these settings.

The group relation can be seen as an extension of the dynamic between Ego and Alter to a plurality of subjects, but of course, complexity increases. Some examples come to mind, such as the case of the "reflecting team" used in mental health services that conduct therapy with individuals and families 
in difficulty ${ }^{2}$ or the case of the "family group conferences" (Seikkula and Arnkil 2006) ${ }^{3}$ that work to help parents manage problems with their children in situations of risk by building interactive networks between them from which a relationality can emerge that supports the parents in their tasks. In these group dynamics, we can see that the network connecting participants is formed on the basis of a "social molecule" (about which I will say more later), which is formed by the modality of relational reflexivity adopted by the participants who, anyway, have different individual modes of reflexivity.

\section{The relation as generative mechanism}

Social morphogenesis begins within relations, and it is through relations that new social forms are generated. It is in the social relation's form ${ }^{4}$ that compatibilities, contradictions, and complementarities ${ }^{5}$ between the elements that compose the relation are, or are not, realised in varying ways and degrees.

When the social relation approximates to a habitus, reciprocal action takes on a reproductive character, based on negative feedback. Social morphogenesis comes about when the relation involves the reflexivity of subjects and entails positive feedback, in particular, relational feedbacks (Donati 2015b).

2 The "reflecting team" is a method of social work aimed at providing relational services to people in difficulty or at risk through the building of a relational network between professionals and all the people involved in a particular issue (Andersen 1991). The team removes barriers between therapists and clients, lets everyone participate in a dialogue in which there is the attempt to achieve a change in the situation by continuously questioning the roles and relations among all the participants. Operating in this way, the professionals encounter people in a state of need without pre-existing hypotheses, engage in a conversation that probes what has still not been seen or thought, in a form of communication that aims to let an alternative understanding emerge regarding what has been defined as problematic. To the extent that the network operates with these relational modalities, possibilities for change are opened up. These possibilities are evaluated in light of therapeutic goals and, thus, we are in the presence of what I call a relational system of observation, diagnosis, and relational guidance.

3 A family group conference (FGC) is a decision-making and planning process in which the "wider family group" (parents, kin, friends, neighbours, other families) makes plans and decisions for children and young people who have been identified either by the family themselves or by service providers as being at risk and in need of intervention that will safeguard and promote their welfare. It is possible to define an FGC as a relational service because it is based on a participatory approach in which social services work together with parents, children, and other important relations to find the right way to care and protect the child by stimulating the reflexivity of the people involved and their relations.

4 The form of the relation answers the question: "What type of relation do we have/want between us?"

5 According to what Archer theorized (1988: 219-226, 258-273). 
A relational feedback, differently from individual positive feedback (which is an individual variation introduced by Ego into the relationship with Alter), is a positive feedback working on the relational structure of the participants' network. It operates when the subjects perceive that their relational structure is like a reality that influences them for good or for evil as relational subjects. Then, the agents can reproduce or modify the relational structure of their network. They can stabilise it or destroy it, depending on the kind of reflexivity that governs the feedbacks, and of course, reflexivity can also be blocked.

In general, the relation is a generative mechanism to the extent that it contains reflexivity, and the latter makes the black box non-trivial. That is why the emergent relational structure has qualities and properties that "exceed" social interactions (which have an événemential character: they are pure "events"). Among various properties and powers belonging to the relational dynamics leading to Time4 (Figure 4.1), I would like to emphasise two that are connected to each other.

Firstly, the social relation is intrinsically reflexive, in the sense that it "always circles back" on to the subjects that are in the relation. Naturally, reflexivity can be minimal, impeded, distorted, or fractured and in that case so will be the relationality among the agents/actors. Precisely because they "exist in relation," agents/actors must think and act focusing on their relationship beyond individual intentions or actions. The structure of the relation is reflexive in that the axis of the refero is not only a symbolic reference to the goal that Ego and Alter intend to realise (i.e. define which relationship to have between them), but it is also a continuous bring back (referre) what emerged from their prior interactions to their new resolutions. In case, there is no solution to the problem of how to define the mutual relationship, there will only be a (contingent) interaction, not an established social relation.

In my opinion, it is in the quality of the reflexivity put in place by the agents that the ethical value of the relationship resides, to the extent that the relationship requires a "response" (responsibility), that is, being responsible to oneself and to others for the results of interactions. Obviously, reflexivity will have quite different characteristics from one relation to another and from one context to another. In the market, for example, things happen very differently than in the family. In any case, the reflexive character of the relation is decisive for social dynamics. We can speak of the relation as the "reflexive mechanism" of the social realm.

Secondly, and in parallel, the social relation can never be purely mechanical because it has a ternary, not binary, structure. Automatic mechanisms are binary (stimulus-response, $0-1$, yes/no) ${ }^{6}$ and do not have purposes (read: desires), since they perform functions. The social relation, instead, contains

6 As Simmel (1989: 258) claims, "The formula of the purpose is ternary, that of the mechanism [is] only binary." 
a finalism - one that may or may not be realised because of other countervailing mechanisms that are at play. Insofar as the relationship operates with a finalism it constitutes a particular generative mechanism, which can obviously produce very different effects. It is not a given that the relation's finalism corresponds to the ends of the singular subjects who are in relation, even when they are in agreement. On the contrary, it is highly probable that the outcome caused by the relation is distanced from the particular goals (wishes, expectations, etc.) of the singular subjects in relation precisely in that it is an emergent effect that mediates between subjects. The finality may be simply to enjoy the products of their relationships. In any case, to say that relations have an ethical dimension, simply means that relations have a moral value since what they mean to the agents is good or bad, or an inscrutable mixture of both.

Often the actors/agents that appeal to noble "values" such as peace, justice, and solidarity are not efficacious in generating a congruent social structure because they do not see how to give form to the social relation that is meant to realise that value. In order to understand the problem of the relation's form, it is useful to think of it as a "social molecule."

I use the term "molecule" to state that the components of a social relation have to connect in a peculiar way in order to produce an emergent effect endowed with a certain stability and causal powers, if the social morphogenesis has to generate an elaborated structure. Although a certain degree of contingency can and should be introduced in the process through which an emergent is generated, not all possible combinations of the relational components can be effective in producing a specific kind of social relation. In general, every social sphere (like an organisation, an association, a public office, a school, a hospital, a family, a pub, etc.) is identified by a speciesspecific kind of social relationality, with its associated effects.

In talking about the social relation as a molecule of the social world, I think that this is more than a metaphor for the molecule which exists in the physical-chemical world. In the latter, a molecule (elementary or composed) is the smallest particle which retains the characteristics and specific properties of a physical substance and is capable of autonomous existence (as long as it can). Something similar happens - as loose as the analogy may be with regard to the social relation which characterises a specific social sphere or context or fabric. We can think of the social molecule of a youth gang, a neighbourhood, a voluntary association, a firm, a school, a cooperative, a bank, and so on.

Take the example of schools. Of course, every school is different from any other. But, after all, each one is a school, not a family or a corporation. School system can be very different from one another, for example centralised in France and decentralised in the United Kingdom. But does that mean that all schools have nothing in common? Why do we call them "schools"? Is that only a nominalist convention? When I argue that schools are characterised by a specific social molecule, I am referring to the fact that 
they have in common a system of relationships that distinguishes them as schools from any other type of social relationship systems. But of course, the molecule is different for each school, just as all humans have the same DNA in common but each one has a unique composition.

\section{The social relation as molecule of the social}

The social realm tends to organise itself in "molecules" that - if stabilised generate the social structures that characterise a context. If I go into a pub, the social context that I find there envisions certain goals (I cannot ask to buy a bicycle), certain means for achieving these goals, certain rules and values, not to mention certain combinations of these. There are pubs for young people, who go there to get a drink and find friends. And there are pubs for older people, who go there to play cards. In this, the social molecule of the pub is materialised; it can also, obviously, be changed, in which case the social molecule meets a process of social morphogenesis.

Let us see what is meant by the assertion that we can think of the social relation as a "molecule" whose elements, with their internal combinations, confer on it particular properties and powers. I must repeat that the reality of an established social relation (when it is an emergent effect, an elaborated structure) continues to be activity dependent, since there is no deus ex machina that makes it exist independently of the subjects. It exists outsides the subjects, but cannot work without them.

We can briefly recall the specific social molecules that characterise the different types of society.

A tribe or clan exists in as much as whoever is part of it feels bound within the "tribal molecule," that is embedded in that organisational principle of the tribal or clan structure that is the same for everyone and does not leave margins for variability. The assertion by Durkheim, according to which the collective conscience coincides point by point with the individual conscience, cannot be absolutised because collective representations cannot completely block the reflexivity of all individuals. There is always the possibility that some member(s) of the tribe can avoid the coercion of the "collective conscience." This possibility does not detract from the fact that the collective mechanisms are at work: to claim that a tribe implies a peculiar social molecule is equivalent to claim that in tribal societies the social differentiation is segmentary, and not stratified or functional, let alone relational in the sense intended here.

Pre-modern traditional societies are generally "stratified." In this arrangement, each social stratum has its own "social molecule," the molecule of that specific social stratum (or class). The social relations in the medieval aristocratic class have a social molecule that is different from the professional or artisan classes organised in corporations or guilds (physicians, notaries, carpenters, butchers, etc.), and from other classes (think of the peasants) as well. Each stratum or corporation has its own social molecule 
in terms of the aims, means, norms, and values of the social relationship that each individual belonging to this stratum must comply with.

Modern societies break away from the molecular structure of the preexisting social strata and inaugurate a type of social molecule that, in its own properties and powers, is completely different as compared with all those that came before. This is a molecule that tends to nullify its morphostatic character. The form of the modern molecule is based on the principle of functional differentiation as its basic principle. In late modernity, functional differentiation opens up to the guiding value of contingency (the value of the plural) as the superordinate value of action, ${ }^{7}$ which involves the maximum possible variability and variations of social relations.

In fact, we say that a society is "more modern" to the degree to which its social molecule promotes pluralism, that is an increasing pluralisation of all social forms as its directive distinction. In postmodernity, the process is radicalised. The social molecule appears as an aggregation/combination of elements that is a "form without form," in the sense that postmodernity's social forms reject any kind of "canon," predefined standard, truth, or stiff identity. It does this on the basis of principles of freedom and equality among all participants. In a certain sense, postmodernity can be defined as a form of society in which the structures of social relations are intrinsically (i.e. normatively) morphogenetic in that social morphogenesis is simultaneously the value and the norm that guides all of society in all its expressions. In the postmodern form, social identity is like that of the protagonist in Philip Dick's novel $A$ Scanner Darkly who, in continuously adopting different identities, loses the sense of self.

The goals and means of the social relation follow an evaluative criterion that opens the door to the world of the possible. This criterion consists in the continual creation of variations and variability. This process is extended to all social spheres, albeit gradually. It affects, at different and non-sequential times, all social institutions, like the political system, the economic institutions, the family, the school. Social processes make use of generalised symbolic media that can operate only on condition of being able to realise the normative value of maximum openness to the possible ("being politically correct" is its basic norm). The slogan is to create ever new and ever greater opportunities as goods in themselves for the individuals irrespective of their relational consequences, except of course for damages to others. In the first place, money and political power do this. The same thing is required of all other generalised symbolic media, in particular the use of influence to form public opinion.

We can represent the social relation as a molecule with a chart (Figure 4.2) indicating that the relation's structure consists of four elements and their reciprocal links. The four elements are: $(\mathrm{G})$ the concern/interest selected 


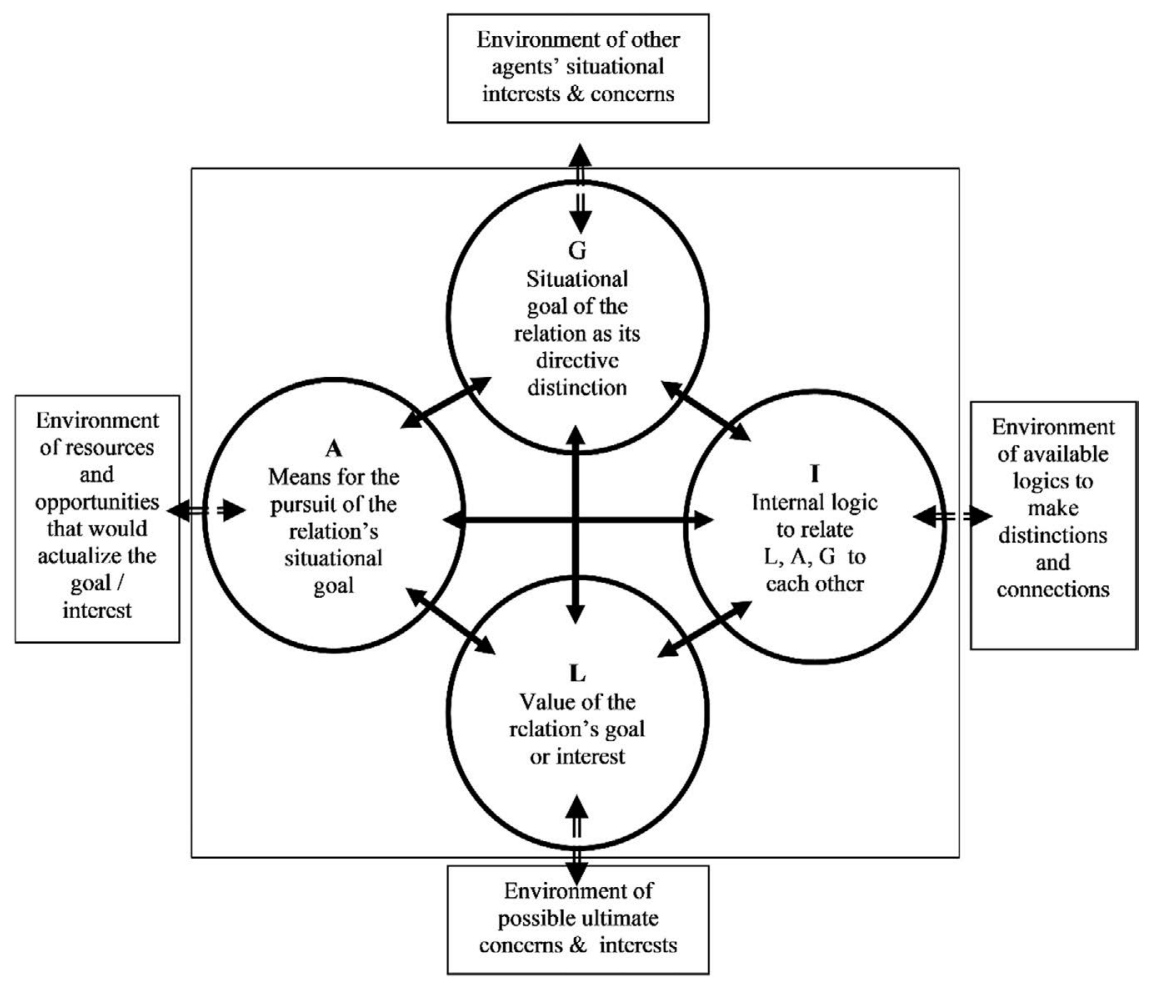

Figure 4.2 The model of the social relation as a "molecule of the social" and its environments

Source: Author.

or selectable as directive distinction for the relation; (L) the cultural value attributed to the relation's concern/interest; (A) the means available to pursue the relation's concern/interest; (I) the logic of relating (through distinctions and connections) the other elements $\mathrm{L}, \mathrm{A}$, and $\mathrm{G}$.

For example, if we want to carry out a survey on how individuals choose to make a couple, what is at stake is the relationship with the partner. The elements of this relationship could be detected through the answers to the following questions: $(\mathrm{G})$ which people are interested in living as a couple, or, conversely, do not consider such relationship as an interesting purpose for them?; (L) what value to attribute to that relationship? (it could be a marriage, a simple cohabitation, a temporary experience); (A) what means are considered suitable to achieve the goal?; (I) how do individuals put these elements together in order to configure an acceptable modus vivendi for them? From all this, we can draw, with various techniques, a profile of the various ways of structuring the couple relationship in a certain population. 
Each element of the relationship has a border with a specific environment. The environments are as follows: (L) the cultural value attributed to the selected social relation borders on the environment of the possible ultimate concerns/interests that can be attributed to that relation; $(\mathrm{G})$ the situational goal selected or selectable as directive distinction for the relation borders on the environment the other agents' situational goals (the environment of the interests pursued by others); (A) the means adopted for getting the relational goal borders on the environment of the possible resources and opportunities that would actualise the situational goal; (I) the norm chosen in order to relate $\mathrm{L}, \mathrm{A}, \mathrm{G}$ borders on the environment of the available logics to make distinctions and connections so to perform the relation.

It should be noticed that the component called "norms" is to be understood not as a legal constraint, but as the logic (and therefore rules) of relatedness between the elements of the social relation which enables them to connect in some way and produce the emergent effect (in this regard, norms are not only restrictions, but also enablements). Norms can foster innovation. Indeed, such norms are required. Examples abound. The field of norms introduced by law in order to change family patterns is very rich in this respect: for instance, to make the parental leave compulsory for the father (together or instead of the parental leave for the mother) innovates family relations. The field of social services and social policies is another one: for example, introducing the norm of "governing by networking" leads many welfare services to be changed from a vertical structure to horizontal configurations characterised by forms of circular participation and dialogical settings.

On the basis of this conceptualisation, we can feature the social molecule produced by modernity in the following way (Figure 4.3). It is constituted by four base elements that are combined together:

(G) The situational target or goal of the social relation is the directive distinction that works to select a variation as a broadening of opportunities; to produce social morphogenesis, the variation must be freed from all ascriptive constraints (i.e. it must be maximally contingent).

(A) The means for achieving this goal can be of the most diverse kinds, but what is essential is that they be treated as "money" because money is the generalised symbolic means of interchange that makes it possible to render all objects equivalent, depriving them of their constraints; as a matter of fact, money is the trigger of typically modern relationality, which renders social relations indifferent, making their ascriptive character and intrinsic quality disappear.

(I) The norms (internal logic) of the modern social molecule are rules that must promote the production of variety; they are typically acquisitive which means that they valorise competition rather than concomitant complementarities. 


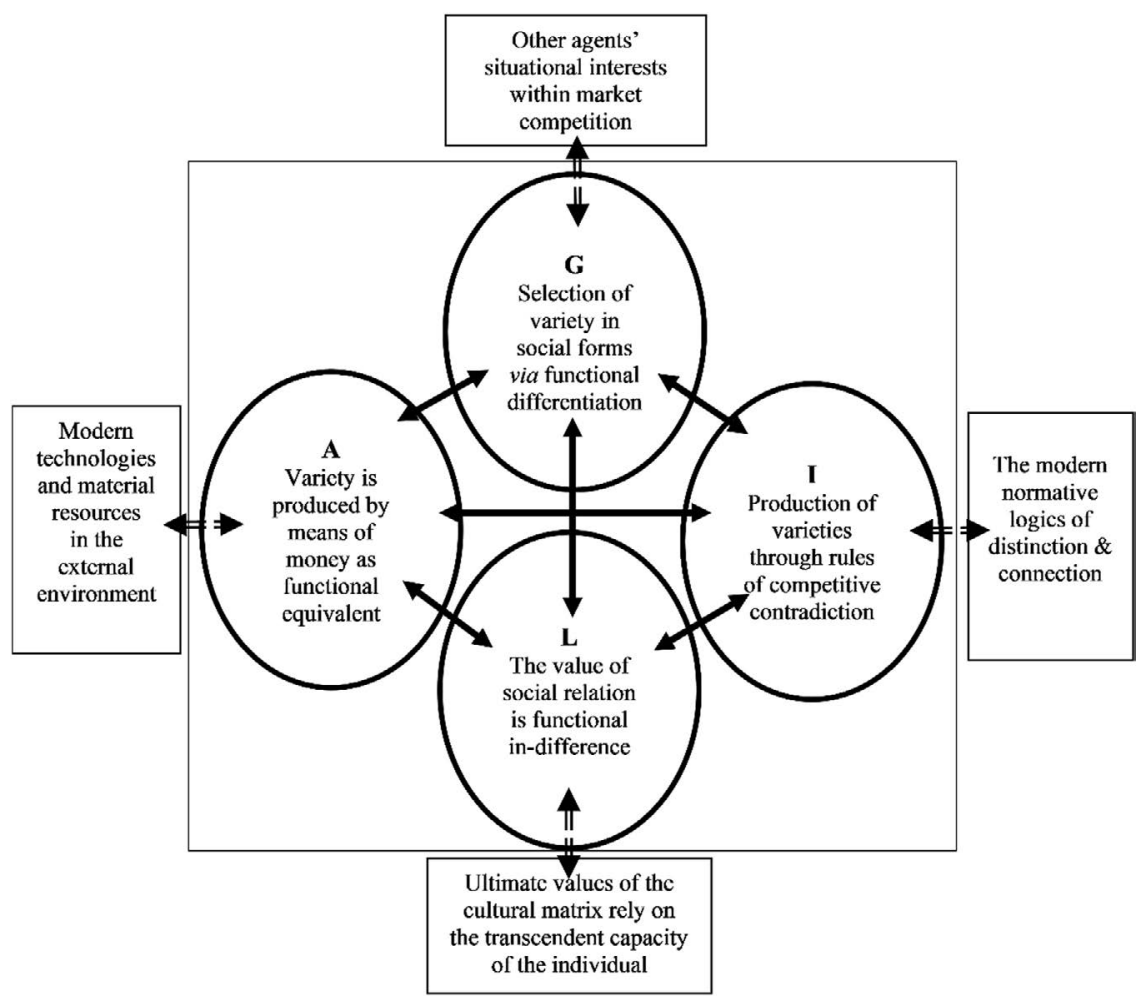

Figure 4.3 The structure of the social relation (social molecule) typical of modernity Source: Author.

(L) The value of the relation's interest, working as guiding distinction, is its indifference towards unconditional values (i.e. its polytheism of values, as Max Weber would say); this is to say that the relation assesses reality on the basis of values that are always fungible with other values; the culture of this society (society of individuals) is characterised by relativism and decontextualisation of relations; it is fed by a theological matrix for which transcendence belongs to the individual's skills, to her self-affirmation, and is non-relational (Donati 2010).

Concerning the environments of the typical modern social molecule, Figure 4.3 tells us that $(\mathrm{G})$ the selection of variety in order to change social structures and institutions is related to an environment where other relevant people make selections of their interests in a different way, from which the competition arises; (I) the rules combining the elements are related to 
an environment in which the binary logics of distinction and connection typical of modernity are found; (A) money serves as a means to address relationships with the external environment where physical, scientific, and technological resources are found; (L) the cultural value is related to a theological matrix that derives from Protestantism considered as the engine of an evolutionary process of individuation and rationalisation (as portrayed by Parsons 1967).

If we observe things in this way, we can come to appreciate the truth and, at the same time, the fallacy of Luhmann's thesis. It is well known that Luhmann (1995) maintained that the adaptive function of society, that is the instrumental function performed by money as a generalised symbolic means of communication, is the dominant and specific function of the modern social system, in contrast to the systems of pre-modern societies. A society - Luhmann (1976: 517) asserts - that gives institutional primacy to monetary mechanisms (i.e. on a systemic level, to its economy) will therefore orient itself towards an "open" future and will conceive of itself as progressive (as moving ahead). In his opinion, modernity consists precisely in the creation of a continuous variability due to the fact that functional primacy is given to the economy, which disengages money from specific values and social norms (for more details, see Donati 2011a: 150-152).

Luhmann takes up and brings to its extreme consequences the perspective of Georg Simmel according to which the more the objects are exchangeable, the more the same objects are perceived as replaceable, and therefore they lose their specific qualities. They become abstract entities. Money represents the purest form of these exchanges, a form of interaction that is separate from the content of the interaction itself. Consequently, in the monetary economy, all relationships between people tend to be considered as measurable and able to be calculated, and the calculation prevails over sentiment. I guess that those relationist sociologies that reduce relationships to pure interactions and transactions, willingly or unwillingly, reflect this culture of money as a means of making all relationships equivalent, including those between humans and non-human entities, for example robots.

So far, Figure 4.3 is in agreement with Luhmann's theory. Where my relational theory disagrees with Luhmann's theory is that in his opinion, modernity does not have a standard norm, while I believe that the modern relationality also depends on a standard of normative value. This standard model enhances a type of "pure relationality" (that of money, which is not tied to anything) understood as the possibility that social relationships may be or become immune from any social bond. In this case, it is not money like all genres of means - that makes society "always otherwise possible" (as Luhmann claims), but it is, instead, the cultural value that is attributed to social relations in the typical molecule of modernity, and related social norms. It is this normative value that legitimises the use of money as a universal converter, that is, the use of money as the abstract equivalent of everything, and even of human persons. 


\section{From modern to aftermodern relationality}

We might wonder: when is it that the social morphogenesis of modernity comes about? From the point of view of relational sociology, this happens when the relationality unique to its social molecule finds itself confronting a type and degree of contingency that it is no longer able to manage. Society (as defined here) approaches a breaking point in which agents/actors experience increasing failures. The collapse of the social molecule that characterises the structures of the acquisitive society becomes possible. This happens in the spheres experiencing the most radical processes of modernisation. Many emergent phenomena signal the advent of a turning point, which is indicated by the formation of another social molecule, of an aftermodern type, beginning from the sphere of cultural latency, that is, by a change in values that support new situational goals. Obviously, there is a modernity that continues and a modernity that collapses. These processes are represented in Figure 4.4.

The transition begins at a breaking point where social disasters induced by the modernisation processes cannot be solved by modernity itself (it is the concept of "reflexive modernization" proposed by Beck, Bonss, and Lau 2003). The turning point for the change of the relationship as a social molecule occurs through the adoption of a principle of suprafunctional non-equivalence. This turning point initiates the processes of social morphogenesis that lead to the emergence of the aftermodern social molecule. This does not prevent some processes of neo-modernisation from continuing to persist, while in any case, the modern social molecule degrades in increasingly wider areas of society.

The collapse of the modern relation can be described as the fading of the competitive contradiction between culture and social structure based on an individualistic transcendental matrix. This configuration is followed by the birth of experiences in which a different synergy emerges between a new culture

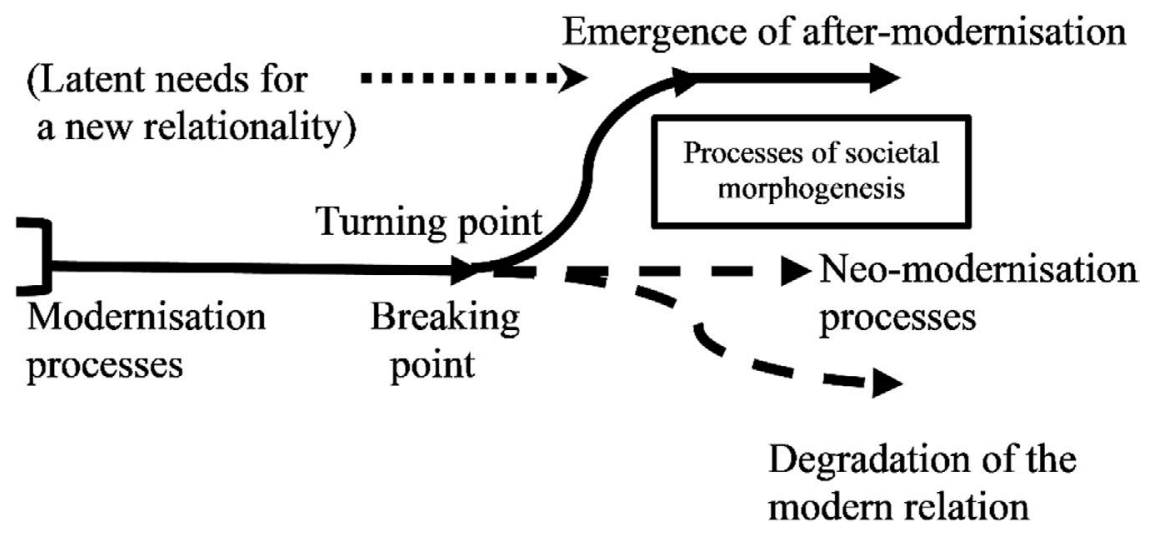

Figure 4.4 The transition from modernity to aftermodernity

Source: Author. 


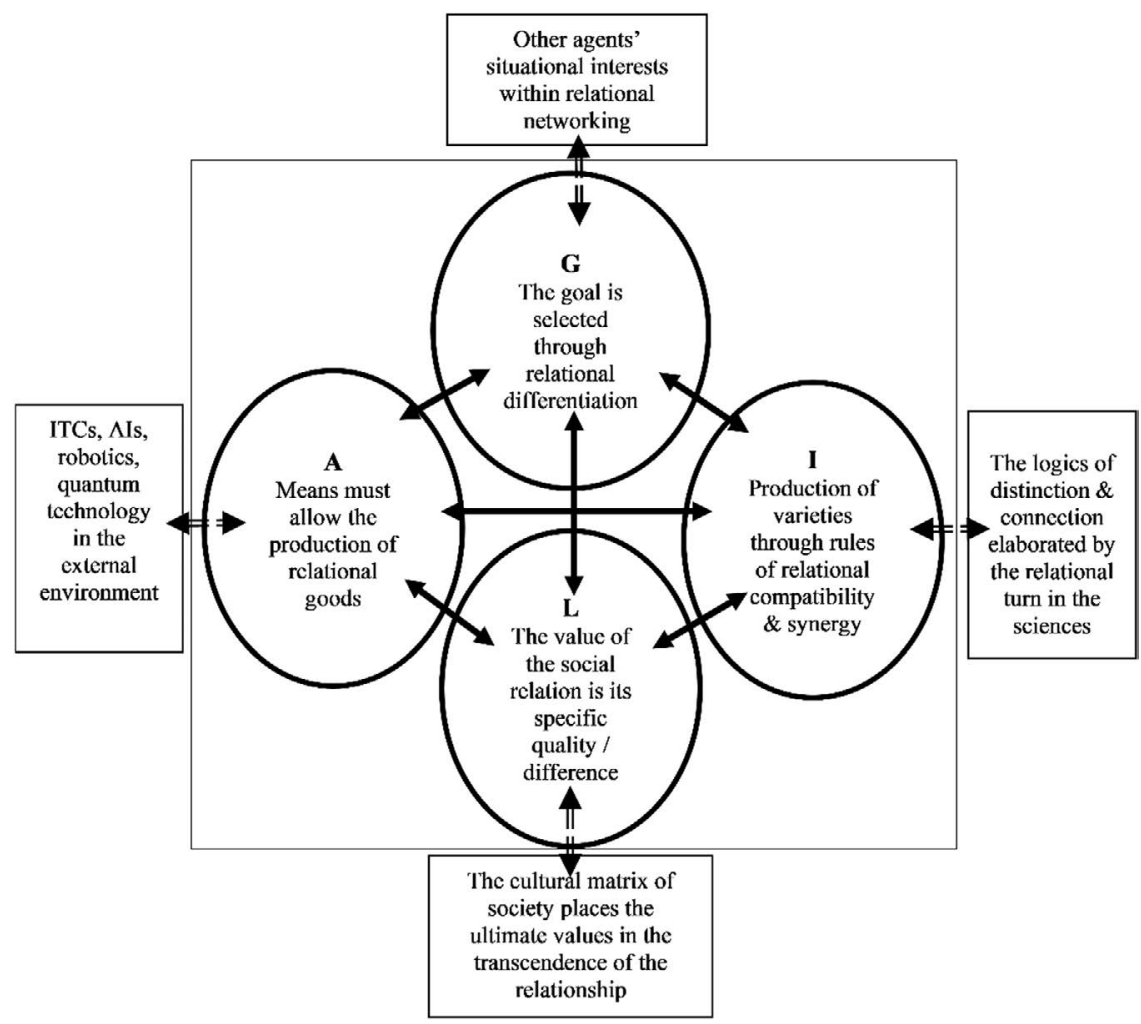

Figure 4.5 The molecular structure of the social relation typical of an aftermodern society

Source: Author.

and a new structure, both characterised by attributing a relational meaning to values as well as social roles, functions, and performances (Figure 4.5).

Rushworth and Schluter (2011: 2-3) write, "This means learning to see life from the perspective of relationships, as opposed to seeing it from the viewpoint of materialism or individualism. Instead of assuming that income or profit should generally be the ultimate goal for personal, corporate or government decisions, we argue for relational wellbeing instead since ultimately our relationships are what matter most in life. Learning to think relationally calls for a Copernican revolution: instead of placing material wealth, or individual rights and freedom, at the centre of our metaphysical solar system, with all other things - including relationships revolving around them, we place relationships at the centre, to reflect better what we ultimately value. As an example, take the decision to buy a microwave oven: we may consider the decision financially (can I afford it?), or spatially (is there room in the kitchen?), or environmentally (how does this affect my carbon footprint?) - but what about relationally? Having a 
microwave could either enhance or lower relational wellbeing in the household. Reducing the time spent on preparing food could either permit more time for talking together over the meal, or else lead to family members eating at different times and not talking together at all. Looking at the decision through a relational lens will bring this dimension into perspective."

The structural principle of the passage to aftermodernity is that of a social molecule that is no longer based upon functional equivalence but is based upon a principle of non-fungibility of social relations. Figure 4.5 illustrates the features of the emergent molecule:

(G) The social relation's target/goal is to select variations according to the type and degree of relationality that they entail, that is through relational (not functional) differentiation, with a view to producing relational goods.

(A) The means for achieving the goal can be extremely diverse, but they must be such as to allow for the production of relational goods; they should promote a network of social exchanges that confer a relationally satisfying identity upon the agents/actors.

(I) The logic of the aftermodern social molecule is relational, meaning that it promotes the rules that coordinate values, means, and situational purposes in order to respect the non-fungibility of social relations; in short, relations cannot be indifferent or made equivalent.

(L) The value attributed to the relation is chosen on the basis of the meaningful experiences that the agent can obtain from that relation in contrast to what can be offered by other types of relations; in short, the cultural matrix is relational rather than individualistic.

Confronted with society's possible collapse, that is with a crisis in its integration on both social and system level, significant parts of society activate morphogenetic processes that consist in creating networks of relations in which the functionalist principle is replaced by suprafunctional principles. Money is replaced by other generalised means of interchange and communication, or by other generalised means of social relationality. For instance, money can be replaced with time (see the experience of the time banks). The cultural matrix changes in order to acknowledge and promote the value of social relations as the distinctive criterion of the new social molecule in which the means no longer have a pure functional primacy. The new molecule gains ground if and to the extent that the primacy of the adaptive function is replaced by the criterion of the intrinsic value of each social relationality.

\section{Where is capitalism going to?}

My differential relational sociology differs from other relational sociologies because it points out that the peculiar/differential constitution of each society, each social sphere, and each social system is based upon the different 
(sui generis) structure of its "founding social relation." ${ }^{8}$ In particular, this theory argues that the likelihood of generating a new emergent and stable social formation increases when (i) each agent in a relationally shared context believes that the others view her/him as a relational subject, (ii) can distinguish between actions producing relational goods or relational evils, (iii) anticipates limited disapproval from others that can lead to positive feedbacks (i.e. an unmanaged increase in other divergent relationships), (iv) is positively motivated to adopt convergent complementarities with the others through relational feedbacks, and (v) can rely on previous experiences of contexts producing relational goods or evils.

To better understand this perspective, it is worth returning to the already mentioned analogy, albeit very loose, between a physical molecule and a social molecule. In both cases, the word "molecule" is used in order to highlight the fact that a structured set of elements and their reciprocal relations identifies the peculiar qualities and powers of a matter or substance, which of course is ontologically wholly different in the physical and social orders: it is basically fixed in the natural order (although not as much as it was believed in the past), while it is highly contingent and interactive in the social order.

The word "substance" is here used by taking into account what Wendy Bottero writes on relationality, when, criticising Bourdieu's approach to relationality, she speaks of "the substance of social interaction" (Bottero 2009: 399) and "the nature of social networks" (ibid: 417).

Whilst Bourdieu prefers a "relational" rather than a "substantialist" reading of social position, his retreat from the substance of social interaction creates a number of problems in his explanatory framework. There are three issues here. Firstly, by stressing the relational properties of social position, Bourdieu sometimes places an undue reliance on homologies between structurally equivalent positions to explain social action, without sufficiently attending to the substance of those social positions. Secondly, many of Bourdieu's core concepts - habitus, field, and, more generally, social space - depend upon assumptions about their interactional properties which are left unexamined. Key elements of Bourdieu's conceptual framework presuppose the prevalence of a particular network pattern homophily, where agents associate on the basis of social similarity. But the significance of homophily for Bourdieu's account of social practice is never fully confronted, and is treated as a theoretical a priori on which concepts like the habitus sit. However, the existence of homophily cannot simply be assumed, and to adequately situate Bourdieu's account of the habitus (and field) greater attention must be paid to the substance and patterning of social networks. Thirdly, by "bracketing off" the concrete nature of social

8 Following Hegel, Günther (1976: 349) talks of a balance between exchange and order so to combine them in a new codifiable principle that may be called "founding relation" (Grundverhältnis or "basic relationship"). 
networks as a feature of social space, Bourdieu ignores the variable interactional properties of that space, and so downplays the intersubjective character of practice (Bottero 2009: 403-404).

In my view, such substance exists in so far as there is a covalent bonding between the elements or parts that generate the substance with its particular qualities and powers. By covalent bonding, I mean the stable bond of attractive and repulsive forces between agents who share elements of their actions. The nature of a social network is its molecular quality, that is the quality of its constituent social molecule, for instance, the molecule which constitutes a company, a voluntary association, a political party, a cooperative, a kinship, etc. as a sui generis network. These different forms of association are obviously contingent and a matter of degree. What is essentially relevant is their peculiarity from the viewpoint of the qualities and causal powers which influence the interactions in a relationally contested field or organisation. Let me explain a bit more.

In chemistry, a molecule is defined as an electrically neutral group of two or more atoms held together by covalent chemical bonds. In sociology, I argue, we can speak of a social molecule as a group of elements held together by co-relations which form a sui generis social bond. Since social morphogenesis, unlike the chemical or biological, is open to evolution and not predetermined, in the same vein, we can say that social molecules are not predetermined and are open to changes, which anyway are not casual or indeterminate, precisely because the elements of the molecule and their reciprocal relations shape a peculiar pattern that distinguishes one social sphere (a "relational order") from another.

The social molecule, then, unlike the molecule in physical nature, is not a pre-established composition, determining which other components it can "mix" with, but it is a structural and cultural configuration of elements acted by people and emerging from their interactions (the interactional order). The latter can change the molecule characterising the stuff of a social formation or context, but what the word "molecule" wants to underline is that the qualities and powers of that social formation depend on the characters of its basic relationality. This is, I guess, what we mean when we talk about a "tribal relation," a "feudal relation," a "capitalist relation," a "third sector relation," a "market relation," a "teacher-pupil relation," a "professional relation," a "family relation," an "internet relation," a "therapeutic relation," a "neighbourhood relation," a "friendship relation," and so on and so forth.

In society, unlike in the physical order, the spheres of social relations are neither like a gas, or a liquid, or a solid (in a gas, the molecules move freely and are independent of each other, in a liquid the molecules are loosely attached, and in a solid the bonds are strong and hold the molecules together). The social fabric is not like that, although many speak of some similarities. What I call social molecules are patterned emergents in the social realm. They can emerge through morphostatic or morphogenetic 
processes and are structured in a way or another due to their internal relational constitution. Their elements interact and establish relations of interdependence between them and with their respective environments in a setting which is relationally contested because the actors/agents intend to influence the peculiarities of the kind of molecule they are engaged to produce (e.g. a different form of capitalism, friendship, school, family, care, and service).

When we talk about capitalism, we talk about a socio-historical formation in which the economic (analytical) dimension of social relations rules the other dimensions within all those social relations that characterise this social formation. It is highly misleading to separate economic and social relations as if they were two empirically different kinds of realities, as some scholars do. To claim that, under capitalism, economic relations can be "conceived as a particular kind of social relations nested, in a way that is supportive and subversive at the same time, into more encompassing social relations" (Streeck 2012: 4, italics mine) leads to conceive of economic relations as a sort of subset of social relations, while they are fully social relations, and hence leads to the confusing position of having to "show how economic relations upon closer inspection turn out to be social ones" (ibid).

The fact that economic relations mould an entire historical formation has to be understood not on the basis of their dominance over other noneconomic relations, but on the basis of the fact that there is a peculiar structure (what I call "social molecule") which pervades all spheres of society, that is economy, politics, laws, lifeworlds, styles of life, families, and even religions as cultural systems. This is what I mean when I say that the quality of a social formation is due to its social molecule. If a new society has to come, it should have a different social molecule. That is what lies below the assertion that unprecedented relational subjects are building such a new social molecule.

This perspective is very instructive about the future of capitalism. If capital, as that specific social relationship of which Marx spoke, has configured the whole of modern society, we must understand if and how the social molecule called the "capitalist relationship" has changed or will be able to change in the future.

Reflecting on Marx's thought, Shaikh (1990: 72) writes, "Taken by itself, a sharp stone is simply a relic of some ancient and inexorable geological process. But appropriated as a cutting instrument, it is a tool or, in a somewhat more murderous vein, a weapon. As a stone, it is a natural object. But as a tool or weapon, it is an eminently social object whose natural form is merely the carrier of the social relations which, so to speak, happen to have seized upon it. Even any particular social object, such as a tool, can enter into many different sets of social relations."

With respect to this correct statement, I observe that when a stone is used as a weapon, social relationships do not change its natural substance (being a stone), but change only its meaning for those who look at it and use it. The stone is now a weapon and no longer a simple physical object, that is it refers 
to social relationships and not to physical relationships. If we change the relational context, we do not change the natural substance of the stone, but we change its sense and its use relations. So, in society, we can always take physical nature in "another way." One wonders: is this also the case for social entities created by mankind? I would respond positively in the case of inert object that cannot act by themselves, but whose operations totally follow the commands of those who use it. For example, the loom used at the beginning of industrialisation, which we find treated in Marx's analyses. One wonders: is it the same for entities such as artificial intelligence (AI) and robots, and in some respects information and communication technologies (ICTs)? In no way. It is clear that, if we apply an AI to the loom, social relationships change. Digital smart technologies do not have a physical substance such as that of stone or loom, but they are human artefacts that involve a relational context in which the interactions between the user and the object change radically. The digital "intelligent" entities at least partially escape the control of those who use them. They open up to a set of possible relationships that are not predictable. Herein lies the discontinuity between the modern (capitalist) economy and the aftermodern digital economy.

If we follow this reasoning, we see that the new economy opens up to the world of possibilities by moving away from physical nature (and from a culture that operates with analogical symbolic code), and therefore we must ask ourselves whether it is still possible to believe that substance and relationships are co-principles of reality, or if we have to completely abandon the substance and speak only of relationships. I believe it is not so. Even digital artefacts (AI, robots, ICTs) have a "substance": they are what they are. Once created and deployed, they are not liquid entities that can take any form. Therefore, the problem is to understand what substance we are talking about in the case of human artefacts. The artefacts I am talking about only admit a certain set of relationships and not others, which means they have their own structure. This structure is their "substance."

For those involved, this substance determines certain social positions and not others. It is up to users to respond reflexively to what those substances tend to determine in user behaviour. A morphogenetic analysis of the process is required (according to the sequence: conditional relational structure [of AI, robot, ICT] $\rightarrow$ interactions of the structure with users and between users $\rightarrow$ change of the initial relational structure). Change is played in interactions and transactions, but the process generates a structure (substance), however, provisional. Therefore, the principle of the co-evolution of substance and relationship still holds true. The mediation of the relational context opens up to logics that may no longer be those of the pure and simple antagonism between capitalists and proletarians predicted by Marx. This is the phenomenon of the hybridisation of social relations, and, with it, of social identities (Donati 2020).

The digital age spreads self-employment and networking. Someone may observe that digital tools (cell phones, tablets, AIs, robots, apps, etc.) are 
still governed by the large multinationals that produce and manage these tools, such as the Silicon Valley multinationals. Undoubtedly, this observation has its share of truth. However, one can no longer speak of an expropriation of the proletarians by a capitalist class, because, to an increasing extent and in the long run, digital technologies will make many economic activities more autonomous, will make them virtual, and therefore work will become the expression of a widespread intelligence and entrepreneurship.

The social framework in which these tools are used gives the worker more room for manoeuvre. A capitalism of social networks emerges in which the fundamental capitalist institutions change their relational structure: the means of production assume a private character only in use, but their ownership depends on the network; the profit motive is limited not only by national governments and international authorities (such as the WTO), but also by a growing competition from non-profit, civil economy, and third sector organisations. The typical competition of the capitalist market persists and also takes on new forms of exploitation of human beings. However, something new changes the previous structure. The capitalist-worker relationship is increasingly mediated by social networks in which there is a relational evaluation made by users of goods and services in terms of reputation and responsibility against companies. In short, a new relationality of social networks creates another capitalism, in which capital becomes - more than before, but differently than before - a human and social relationship (close to what today we call human capital and social capital), in which power is not based essentially on the ownership of the means of production, but on the qualities and properties of the relationships linking people.

\section{Structural effects and relational effects: the added social value of social relations}

I suggest distinguishing between structural effects and relational effects. In the T1-T4 sequence of every social morphogenetic cycle, the structural effects are active at time T1 vis-à-vis agents/actors that begin to interact at time T2. The relational effects intervene in the T2-T3 phase and have as outcome the emergent structural elaboration at time T4.

Structural effects exert their influence on an arena of agents/actors that is a black box: we cannot foresee how the agents/actors will elaborate the influences exerted by the conditioning structure on them.

By their nature, structural effects have a tendency to exert strong pressure to uniform the agents' behaviour irrespective of their individual subjectivity (opinions, values, and reflections). An example of structural effects is the influence that a surgical team exerts on its members. In this setting, the habit of swearing is also practiced by individuals who would normally prefer not to do so. The reason is that there is a collective pressure on everyone due to their having to cope with the stress coming from the intervention's invasiveness and drama (Blau 1960). Likewise, those participating in 
collective mass movements often find themselves behaving in a way that is contrary to their own values inclinations because of the pressure exerted by the mass of people. The same thing happens when someone has to vote openly in a public assembly due to the fear of others' judgement.

Nevertheless, even in the most structured situations, it is rarely the case that all the individuals involved behave in the same way, caving into structural pressures. In such a case, when someone distances him/herself from the group's social behaviour or norm, the relational effects are revealed. In these the agents' freedom is expressed, which is possible because they relate to the situation in a different way on the basis of a meaningful difference: they take on a different cultural model in relation to the purpose of their actions (the L-G axis in Figure 4.5). By enacting different behaviours, they create relational effects that escape the conditioning structure.

In the intermediate $\mathrm{T} 2-\mathrm{T} 3$ phase of social morphogenesis, empirical research can observe the respective contributions of individuals and networks of relations to final outcomes. Individual contributions come from the redefinition that the Self undertakes of its own identity and action when the relational context is changed. The contributions of social networks derive from how others influence the redefinition of Me-We-You in the circle of interactions. The two types of contributions are interconnected. This has been verified, for example, in a study on why and how social interventions succeed in influencing young people belonging to deviant gangs to stop committing crimes (Weaver 2016).

Let us suppose that we pose the question, "Why do some people stop offending and others do not?" To answer this question, we must hypothesise the existence of a black box that operates between the structures that condition people's behaviours, on the one hand, and, on the other, the outcomes of the interactive networks in which the people find themselves. What must be investigated is the way in which the relations are configured in phase T2-T3. The agents/actors have constraints and enablement that come from the outside as well from the network's dynamic. Even though the people act with a certain autonomy, and depending on their reflexivity, it is the opportunities offered by the network that decide whether there will be a cessation of criminal behaviour or not. What is important is to see the sui generis reality of relations that can produce relational goods or evils depending on how they influence individual choices by opening or restricting the possibilities of creating another relational order of reality.

It is in this connection that the issue of the different ways of conceiving social capital comes up. Holists understand social capital as the civic culture that certain institutional structures instill in citizens. Their primary focus is on the ways that cultural norms structure the relationships in which social capital is embedded. Social capital is seen mainly as a structural effect. Methodological individualists emphasise, instead, a micro-sociological definition of social capital as a rational choice of individuals who relate to each other spontaneously. 
In my view, social capital must be understood as a type of social relations that have particular qualities and powers in that they produce, not so much civic conformity or normative reproduction, as relational effects, that is effects that come from the creation of meso level networks of relations in which trust, cooperation, and reciprocity can operate autonomously. Contribution of individuals as such is not enough, nor is the presence of a regulatory cultural system sufficient, but it is necessary to consider the added value of relationships as such.

What needs to be highlighted is the fact that the social network operates with a reflexivity of its own that is characterised in that it entwines agents' internal conversations between them and in this way generates a relational reflexivity between the nodes in the network which exceeds the individual (personal) reflexivity of agents. Examples are what we call team reflexivity, group reflexivity, reflexivity of the We-relation that are not reducible to the single subjects but have their own dynamics (and story), being of different kinds at different moments.

The network's relations change because individuals seek to realise an identity of their own that cannot be achieved except through the network itself (in which the dimension of reflexivity is played out, i.e. Me, We, You). In short, in the intermediate $\mathrm{T} 2-\mathrm{T} 3$ phase of every morphogenetic cycle, the place where change occurs is located in the relational network in which personal reflexivity, social (interpersonal) reflexivity, and system reflectivity meet one another. The subject of this encounter is not the individual as such, but the relational subject.

What relational effects bring to light is the added social value (SV) of social relations that are put into play in phase $\mathrm{T} 2-\mathrm{T} 3$ of the morphogenetic cycle.

\section{The added social value of social relations}

In the abstract, saying "added value" means, briefly, making reference to an increase in something/someone's value. The increase is produced by something/someone that has acted/operated on something/someone that via a causal effect - has increased its value. When this happens through the social relation, we have the added social value of the social relation, that is, the added SV that the social relation confers on something/someone. It is the social relation that valorises something/someone.

The social relation can valorise things, (individual or collective) agents/ actors, or social relations and in this latter case, we are moving towards producing a relational good.

An associative network or a third sector organisation creates because, in producing goods or services (e.g. a service providing care for children, the disabled, and the elderly), it uses a greater quantity and a better quality of social relations as compared to the social relations used by the market or public administrative bureaucracies. If, then, these more numerous and 
better relations are targeted not only at simple service offerings as such but also at relations among the subjects involved in the service, then the service itself becomes a relational good. It becomes a relational service strictly speaking, because the service is made of relationships and not of functional performances.

Saying that a social relation has an added SV means noting the fact that when two entities bond together, they generate a third that goes beyond the properties and powers of each of them and is irreducible to their sum.

The complex of refero-religo that is brought into being generates an added value because it increases the value of the constituents of the relationship, by virtue of the social relation itself. This is an effect that emerges from the relation's properties and causal powers.

This perspective helps us to see the partiality and non-generalisability of the definitions of social relations such as that proposed by Shih (2020:1) according to which the relationship is "a process of mutual constitution that reproduces imagined resemblance, where actors self-identify with others on the basis of a Wittgensteinian network of perceived similarities overlapping." This type of relationships also exist, but in this case, the relationship has little added value, as it is limited to cementing a network of individuals characterised by a reflexivity which is highly dependent on significant others, and therefore little individualised, which is typical of Eastern cultures. These relations can also emerge in the West, but only under the pressure of catastrophic events, such as the Covid-19 pandemic, when everyone is called to conform to the same imagined similarity, that of potential victims of the catastrophe, and therefore to live in heavily regulated social networks.

In order to understand the enhancement process implemented by the relationship, it is necessary to conceptualise the "relation that valorises," given that not all relations have the capacity to produce this emergent effect. And so we ask: how is the relation that confers added value configured?

The SV of an entity X (something, someone, or even a social relation) can be defined as a function of the social relation (r) between two subjects, ego and alter, who carry out the interactions (i) (exchanges or transactions) between them, that is, $\mathrm{SV}(\mathrm{X})=\mathrm{f}\{\mathrm{r}[\mathrm{i}($ ego/alter $)]\}$. More generally, the SV of an entity $\mathrm{X}$ is a function of the network of relations $(\mathrm{Nr})$ among all of the network's subjects (or nodes, Ss) which carry out the interactions (Ii) in the network: $\mathrm{SV}(\mathrm{X})=\mathrm{f}\{\mathrm{Nr}[\mathrm{Ii}(\mathrm{Ss})]\}$.

Networks of relations differ in many ways as to how they treat an entity X. Each network valorises $\mathrm{X}$ in a different way. Certain networks do not valorise $\mathrm{X}$ at all, but exploit it or annihilate it. Only certain networks of relations produce relational goods while other networks produce relational evils.

If we stay with the simple case of the dyadic relation between Ego and Alter, the relation that confers added value can be seen according to four dimensions that are interrelated among themselves. The diagram of Figure 4.6 indicates four boxes in which the four prevailing meanings of the added value of social relations are placed. They are indicated with the letters A 


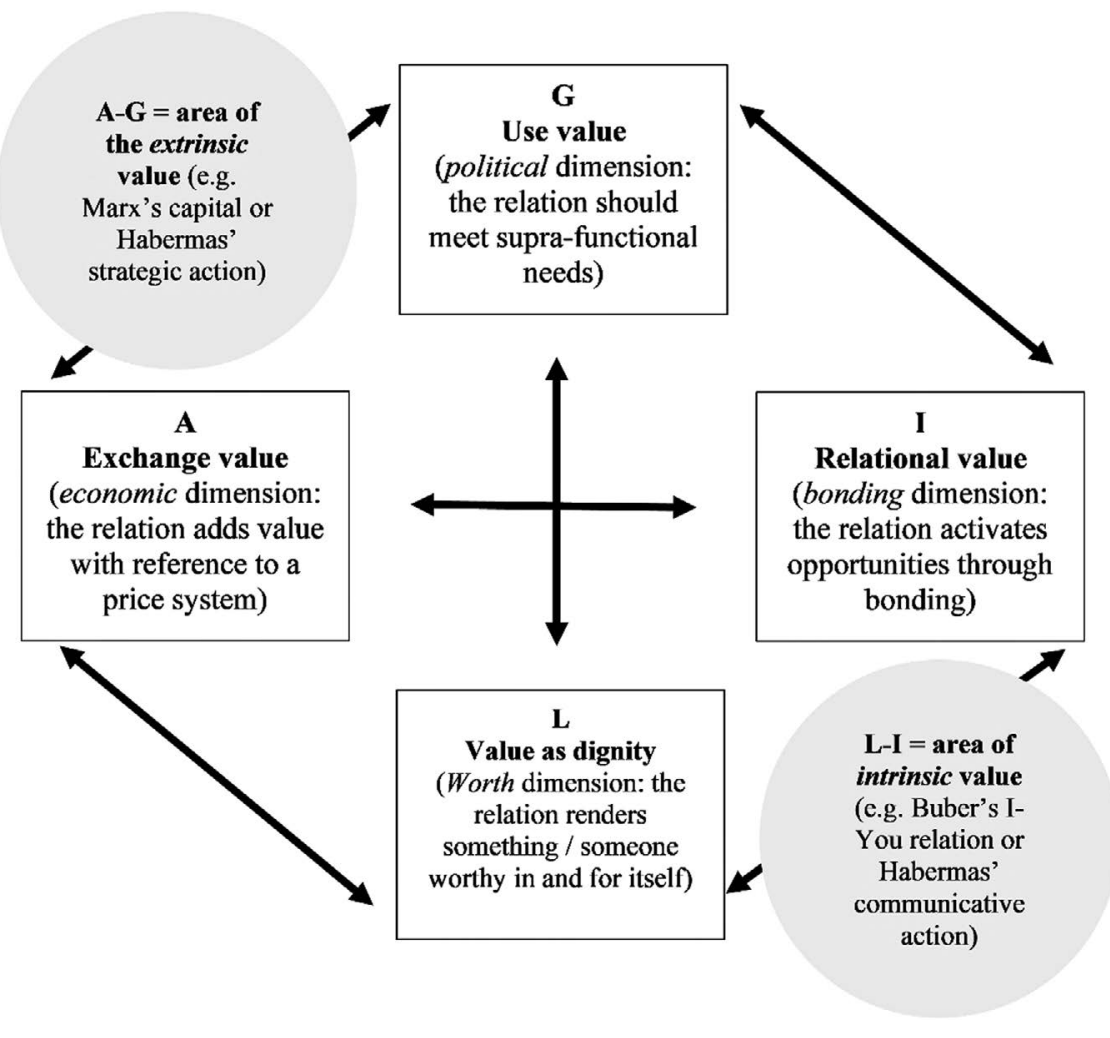

Figure 4.6 The different meanings of the value added by the relation to something/ someone (analytical dimensions of a relational complex)

Source: Author.

(exchange value), the letter $\mathrm{G}$ (use value), letter I (relational value), letter $\mathrm{L}$ (value as dignity). Arrows are drawn between the four boxes to indicate their reciprocal relations. In addition, between $\mathrm{A}$ and $\mathrm{G}$, the area of values in an extrinsic (instrumental) sense is indicated in a circle, and between $\mathrm{L}$ and I, the area of values in an intrinsic sense (as a value in itself). Let us look at the four meanings of "value" more closely.

(A) The relation confers an exchange value when, through the relation, the instrumental value of something/someone is increased with reference to a pricing system (added value in terms of utility).

(G) The relation confers a use value when it can better help satisfy something/someone's fulfilment needs (added value in terms of suprafunctional goals).

(I) The relation has a relational value as a factor that activates connections that increase the subjects' opportunities and capacities (added value in terms of the creation of relations that improve the 
opportunities and/or capacities, qualities, and powers of what/ whom has been put in relation); the relations that we call "social capital" (trust, cooperation, reciprocity) optimise this criterion (added value in terms of bonding).

(L) Something/someone is valorised in its/his/her value of dignity (meaning intrinsic value) when, through the relation, there is an acknowledgement and amplification of the sense of dignity (recognition and respect) that something/someone has in and of itself/ oneself without the possibility that this value (Würde in German, worth in English: Collier 1999) can ever be a matter of negotiation.

These are the analytical dimensions of social "value" that the relation can add to something/someone. In social morphogenetic processes, the value of something/someone is modified. It can be increased or decreased or, indeed, nullified in some or all of its dimensions. Valorisation is an operation of enhancement that comes about through a particular combination among all or some of the four dimensions of Figure 4.6.

For example, in the field of human services, in order to produce added $\mathrm{SV}$ for the well-being of people, the task of social workers is to mobilise a network of social relationships that increase the value of people in dealing with their problems. They can get it if and to the extent that they refer to the ability of social relationships to mobilise opportunities and resources including relationships themselves - which are qualifications for the parties involved.

This type of operation is, in theory, specific to non-profit organisations due to their supposed good motivations. But this better quality of the nonprofit sector is not at all obvious. In order for this to happen, it is necessary that these organisations give priority to valorisation criteria that make reference to the relational value and to the value of dignity (Figure 4.6). This means that there must be the potential for a certain quality and quantity of social capital, that is relations of trust, cooperation, and reciprocity.

These relations have to do with the areas of dignity and relationality (area L-I) of the value, that is, with the area of relations that confer an intrinsic value on something/someone (X) (the box at the bottom right of Figure 4.6). If this area works so as to valorise positive relations, then relational goods can be produced which, in turn, feed social capital in a sequence that is not circular but has the linear character of a time sequence (as we will see later). In this way, the relationality that unfolds in the interaction among actors can increase or decrease the parameters (trust, etc.) of the social capital on which public relational goods depend. If these parameters go below a certain threshold, or even reset to zero, not only is a public relational good not produced, but social capital is also consumed. This is what happens most of the time if the process of valorising something/someone remains confined within the area of exchange value and of use value (area A-G, the box at the top left of Figure 4.6), that is in interactions that privilege the social 
relations' extrinsic value. Many empirical investigations demonstrate this. Suffice it to say that I am referring here to the vast literature on the positive correlations between people's well-being and the quantity and quality of their social relations.

A final annotation. In principle, the area of extrinsic value (area A-G: e.g. Marx's capital or Habermas' strategic action) and the area of intrinsic value (area L-I: e.g. Buber's I-You relation or Habermas' communicative action) are at the antipodes (in Figure 4.6). Nevertheless, in real valorisation processes, there is a differentiation underway depending on the timing and the issue at stake, for which it is advisable not to dichotomise forms of valorisation. It is not a given, for example, that added relational value, which in and of itself has an intrinsic qualitative character, is necessarily incompatible with, let us say, added exchange value, which is extrinsic. On the contrary, the current tendencies to promote cooperation between profit and non-profit entities, for example by establishing organisations of different nature that associate them in order to produce relational goods, are going in just such a direction whereby a process of "composite" valorisation in which all four of the valorisation criteria (in Figure 4.6) work together to create a "total" added SV.

\section{Societal morphogenesis as a surplus of relational forms}

The passage from the modern structure to the aftermodern structure is a morphogenetic transformation of modern capitalistic society through a surplus of new relational forms.

From the previous chapters, we know that modern society is based on the state-market binomial. The social molecule of this structure leaves individuals free to act within an arena of competitive opportunities regulated by political power. The means-target axis (A-G in Figures 4.3 and 4.6) prevails and makes the value and normative components (the L-I axis) of every social relation functional to itself.

Precisely due to the way in which it is configured, this structure neutralises the relationality of civil society where a third sector is operating in an attempt to privilege the values-norms axis. The latter seeks to develop new forms of sociability that have difficulty in becoming institutionalised. There is thus an open conflict between the state-market binomial, on the one side, and a new civil society, on the other. Where is this conflict headed? Is it possible to believe that a society different from the modern one can emerge?

My hypothesis is that a societal morphogenesis is in fact being produced, which is making the third sector emerge in such a way as to alter the lib/lab structure. To verify this hypothesis, it must be demonstrated that in important sectors of society the binary lib/lab structure is being replaced by a triadic structure in which, in addition to the lib and $l a b$ components, the third sector component is gaining ground so as to give rise to a new social molecule that goes towards forming a new relational system on a societal level. 
Many empirical studies have shown that this dynamic is effectively underway.

Let me give some examples referred to the spreading of initiatives "generating the civil" and the emergence of civic entrepreneurs catalysing social innovations. As to the former, one can quote the renewal of self-sustained community foundations, the creation of ethical banks, the adoption of communitarian economies (like the economy of communion), the non-governmental organisations based upon the principles of social solidarity and extended reciprocity between insiders and outsiders, the production of commons on the Internet, the processes of civil constitutionalisation of human rights (Teubner 2012), the civic networks providing welfare services by adopting third sector criteria instead of market criteria or public administration standards (Donati and Colozzi 2001).

As to the emergence of new civic entrepreneurs, the starting point consists in observing that we cannot solve complex horizontal problems with vertical command-and-control solutions. "The speed of change toward third-party provision of all types of public services continues to outpace the ability of most public officials and agencies to manage these collaborations effectively" (Goldsmith, Georges, and Burke 2010: 8). Therefore, "growing cadres of civic entrepreneurs eager for change bring bold interventions that push the bounds of how to address public problems. They are a savvy, motivated, and results-oriented group of individuals who, through disruptive innovations, create opportunities and hope" (ibid: 25). The basic idea that pushes civic entrepreneurship is to combine communal ideals with the efficiency and technological know-how of business, which produces effective community change.

To my mind, the normative impulse supporting social innovation is the following: any civic entrepreneur should enforce a social morphogenetic process which has to change the given hierarchical governmental (conditioning) structure by setting up partnerships among a variety of stakeholders, so to create a complex network which generates social innovations (elaborated structure). "Create an environment for social change" is its slogan.

It is essential to keep in mind that these innovations, and in particular the role played by the third sector, do not replace the state-market binomial, but change it, precisely by causing its morphogenesis. The shift to $a$ new social formation happens through morphogenetic cycles in which the conditioning structure (state-market arrangements at time T1) cannot work without introducing a "third actor," which means that the hierarchical and mercantile codes have to relate to (and interact with) the networking code that forces and binds them to include relational imperatives (in T2-T3). For example, the state has to adopt a social governance style of action, implying more civic participation in designing and implementing its plans, instead of using a pure authoritative style; and the market has to consider the relational dimensions of its modes of production and consumption, implying, among other things, an active, symmetrical, and non-instrumental role for 
the non-profit sector within it. The triangulation state-market-third sector produces then (time T4) an elaborated structure constituted by new configurations at the macro, meso, and micro levels.

If we analyse the social processes through which modernity is passing into crisis and its members (individual and collective) are becoming newly reflexive, we see that emerging social forms are arising in those areas where market and state generate gaps, which cannot be dealt with without changing the social relationality involved in such situations.

Jonathan Rushworth and Michael Schluter (2011) argue that learning to think relationally is the first step towards building a more relational world. They claim that there are three main elements to Relational Thinking: (i) learning to see public policy and personal issues through a relational lens; (ii) changing goals, values, and practices of organisations; and (iii) developing an analytical framework appropriate to relationships. Let us see how this can be achieved with an example.

\section{The instructive example of social cooperation}

From civil society are arising initiatives of a cooperative type which are trying to create a socially responsible economy able to alter the laws of the capitalistic market. Their objective is to keep the law of profit under control by linking money to social constraints. This is exactly what Luhmann held to be an anti-modern action because it does not operate with functional criteria and does not open up to more contingencies; rather, it does the opposite in that it ties the contingencies created by money to prosocial demands. It is a fact that social cooperation truly exists to the extent that cooperative relations do not adopt the law of functional equivalence: they reject the criterion of valorisation based on monetary equivalence. Their ability to thrive is thus always problematic. From an empirical standpoint, cooperative initiatives usually transform into capitalistic organisations. Why?

In my opinion, the success of social cooperation depends on its ability to stabilise an after-capitalistic social molecule. Let us see what this means.

Social cooperation is an organisation characterised by its own social form (molecule) that is based on the following interwoven elements:

(G) The goal is to produce goods that are not sought for reasons of profit but which must have a prosocial end, that is they must satisfy needs that cannot be made subject to exchange for profit.

(A) The means (financial capital, work, technologies) are chosen and treated in a manner appropriate to the pursuit of prosocial goals.

(I) The organisational norms or rules are non-distribution constraint, internal democracy as the cooperative's mode of governance, the joint and several liability of the cooperative's members.

(L) The value (the distinctive criterion that valorises the worth of cooperative organisation) is prosociality, which means to provide benefits not only for those who work in it, but also for the surrounding 
community, and is applied to the mode of production, the destination of goods, and the use of proceeds; sociality, then, means to take care of the people working in it as well as of their families and the community as a whole according to a subsidiarity principle rather than in terms of acquisitive competition.

The integration among these four components is always problematic. It requires a high and appropriate level of reflexivity in managing relations among the elements of the specific social molecule that we call "cooperative form." In particular (see Figure 2.3), system integration (A-G) and social integration (L-I) are difficult to combine according to a concomitant complementarity that is able to connect them efficaciously. When the impulse to create a social cooperative that comes from the sociocultural area (values and norms) does not find complementary correspondents in the system area (means and goals), the cooperative initiative goes into crisis.

This happens because the concomitant complementarity between social and system integration must confront challenges that lead the cooperative to adopt a contingent complementarity. ${ }^{9}$ For example, managers use money for a collective profit, thinking of then investing it for prosocial purposes. But in this way, they abandon the primacy accorded to the value of sociality. The constraints that prevent the contradictions between profit motivation and prosocial ends weaken and lead to competitive contradictions between system requirements and social integration requirements. When this happens, cooperative enterprises become market enterprises.

Throughout history, many social cooperatives have experienced a capitalist drift. The drift in the direction of a capitalist mode of operation can only be avoided if social integration (the value of sociality [L] combined with regulatory norms [I]) manages to make the integration of the system relational (i.e. it manages to use means of production [A] appropriate for prosocial purposes [G]). If the target of producing relational goods, and not other things, must be achieved, all the factors of production (money, labour, technologies) must be assessed in a relational context that organises them so as to produce these goods.

Generalising the discussion, we can say that the creation of "other markets" (not capitalistic markets) depends on introducing a new relationality into the components of the cooperative form (its social molecule). This is precisely what is happening in some sectors of the economy, which, not by chance, are often called "relational" such as the production of a commons

9 For the concepts of constraining contradiction, concomitant complementarity, concomitant compatibility, and contingent complementarity, see Archer (1988: 153-175, 235-237). Contingent complementarity here means a situational logic in which relationships are oriented towards increasing opportunities at the cultural system level and specialisation at the sociocultural level. 
on the Internet (Bauwens 2008), relational tourism, the relational enterprise, the civil economy (Bruni and Zamagni 2004; Rushworth and Schluter 2011).

The social molecules that escape from modernity have a certain characteristic: in an implicit or explicit way, they orient towards producing relational goods - instead of individual or aggregated goods - through relations. This is precisely the task of social capital. In the international debate on this topic, at times social capital is considered as an explanans and at other times as an explanandum. The solution that I propose lies in observing social capital inside a morphogenetic sequence with respect to its products, that is, relational goods.

To clarify this point, I propose a scheme (Figure 4.7) that synthesises my foundational theses according to which social capital is a product of relational goods and, in its turn, is a regenerator of relational goods, or vice versa, relational goods generate social capital which, in turn, regenerates relational goods. This recursivity can be clarified by introducing the morphogenetic scheme that takes into account the temporal phases and the autonomous ("stratified") contribution of each element in the single phases of the process.

Figure 4.7 wants to offer a solution to a long-standing issue: whether it is social capital that generates relational goods or vice versa. The answer is that there is no logical priority of one over the other, but instead a mutual determination between the two that develops in sequences of time cycles.

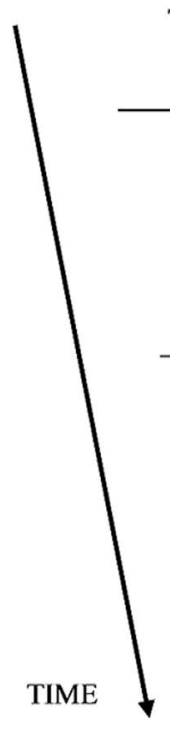

T1 Starting network: there exists (or is created) a network of relations between actors that activates/mobilizes itself in order to organise a social service (this is the design of the intervention which aims to create a relational good)

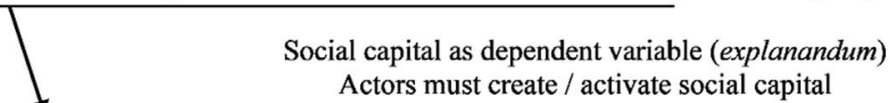

T2 Interactions in the network: the dynamic of the network of relations generates interactions which can produce [or consume] social capital in terms of relations of trust, cooperation, reciprocity [or the opposite] - T3

Social capital as independent variable (explanans) Produced social capital can generate a relational good (on the contrary, the consumption of social capital can generate relational evils)

T4 Emergent network and its effects: the emerging network, endowed with increased social capital, produces the service planned at the start as a relational good (the social intervention is successful), or it fails completely or partially

(a new cycle begins in which network actors can retry the intervention or abandon it)

Figure 4.7 The generation of relational goods through social capital (and vice versa) Source: Author. 
The influence of one on the other depends on the phase in which we observe the social process.

Let us look at an example of the added SV of social capital and the (re) generation of relational goods over time.

Time T1: suppose that a plurality of actors wants to create a day-care service for children or home care for elderly people that has the characteristics of generating relational goods for them. This target can be achieved by mobilising relations among the concerned (formal and informal) caregivers and the individuals who need the service, with particular attention to the primary and secondary networks of those to be assisted. This generates a situation from which more or less social capital can emerge in terms of trust, cooperation, and reciprocity among the actors.

Phase from time T2 to time T3: the fact that more or less social capital is generated depends on the agents and the network that is created and mobilised. Here the agents' and their networks' reflexivity comes into play.

Time T4: if the dynamic in the network interactions of the second phase (T2-T3) has generated more social capital, instead of consuming it, then the initial project of producing a relational good is achieved; otherwise, if, in the second phase, social capital was only consumed or destroyed, relational goods are not produced, and the project fails. After time T4, another morphogenetic cycle will begin.

This framework avoids conflations between relational goods and social capital, keeping them distinct yet also relational with one another.

So we can speak of an added SV of social capital that (i) consists in (re) generating (rather than consuming, annihilating, etc.) relational goods; (ii) can be measured with the associative network's capacity to produce internal and external relations that act in a reflexive manner (Donati and Solci 2011: 101-109) in such way that the shared interpersonal relations are made more efficacious, that is they increase the network's operative efficacy (the strengthening of the target $\mathrm{T}$ applied to the associative network).

Added SV is the difference between the initial situation at time T1 and the situation at the end of the morphogenetic cycle under consideration at time T4. It measures the capacity of an associative network to be efficacious qua association (thus, to not be subject to something/someone other than itself) in that it measures the capacity of a network to produce sociability as an added value (i.e. as the enhancement of its relational parameters: e.g. the degree of reciprocity, cooperation, trust, and affinity). In the event that social capital has decreased, consumed, or destroyed, we will speak of a subtracted SV.

A practical example can be that of the Tagesmütter (the so-called mother of day). This figure can be created with a contract issued by the municipality to the mother of a young child ( $0-3$ years old) who takes on the task of caring for other children (two or three of them) of approximately the same age in her home (it is a family day care). The contract creates a relation among the involved families, which assign themselves a shared 
objective or task (the care of their children, entrusted to the Tagesmütter). In this action of trust and reciprocal cooperation, there is the premise and promise of a relational good. It depends on the second phase (how the interactions among the families go) as to whether their interactions will operate so as to generate, and not consume, social capital. In other words, if a relational context is truly created consisting in trust, cooperation, and reciprocity among the children's parents (who, due to the type of relation established, know and spend time with one another in keeping with qualities and powers that a day-care centre organised in a bureaucratic or mercantile manner would not produce), then social capital generates a network of families in which relational goods flourish; otherwise, the social intervention takes another path.

More generally, organisations can be considered as being of the third sector when they are configured as social spheres that produce relational social inclusion through a virtuous interaction between relational good and social capital. The interaction is virtuous both because it enhances each of them in turn and because it operates with prosocial, civic, or civil finalities.

\section{How social relations realise social morphogenesis}

There exist many and differing types of social morphogenesis. To conclude this chapter, I would like to propose a typology based on the ways in which the conditioning social structure (at time T1) constrains social relations in the intermediate phase (T2-T3) of the morphogenetic cycle. The constraints can have to do with each of the social relations' dimensions (goals, means, norms, values) and also with the relations between these dimensions.

For the sake of simplicity, I propose to classify the ways that social relations are constrained along a continuum that goes from highly determined (highly bound) modalities to highly chaotic (highly unbound) modalities. I then propose to explore an intermediate modality: relationally steered morphogenesis.

1 Highly constrained (highly bound) social morphogenesis starts from a social structure that allows agents/actors fairly limited degrees of freedom because it occurs within cogent and inalterable constraints. Actions are apparently free, but they operate as in a labyrinth from which it is difficult to exit. Lib/lab structures are of this type. They constrain subjects' choices to an arena of limited opportunities by a strict regulation based on the compromise between state $(l a b)$ and market (lib).

2 In contrast, chaotic (highly unbound) social morphogenesis is what happens in a processuality that minimises or annuls the constraints imposed by the structures at the morphogenetic cycle's initial time. Its reason for being is to create relationships that do not have to respond to 
structural constraints. The social molecule annuls the normative component. It affirms relationality as an end in itself. This type of morphogenesis mirrors the relativistic and pragmatist thinking that is typical of the late modernity, which is relationist, not relational, precisely because the relationship must not respond to mutual expectations for a common good, but is an indeterminate sequence of events. Behind this way of thinking and acting, there is the paradigm of the capitalistic market, which generates ever new products and ever new possibilities in an expansive and evolutionistic scenario, without responding to constraints. Thus, it does not need social bonds; on the contrary, it erodes them and has to hollow them out; it creates them and makes them disappear immediately afterwards if it wants to expand. For this reason, it requires adaptation and openness on the part of agents. Individuals must be "open" to any outcome or solution, hoping that it will be positive but with no assurances. Economic institutions, for-profit markets, and corresponding political structures open up possibilities (variations) that are selected on the basis of the fact that they do not demand any constraint apart from combinatory gain, which takes place in the single interaction or, indeed, in each exchange or transaction. A paradigmatic example is the idea of the "pure relation" theorised by Giddens (1992) as paradigmatic for all emergent social relations in post-modern society. In his view, pure relationship means that the relationship exists solely to meet each partner' needs and will continue only so long as it succeeds in doing so.

3 In the intermediate zone between highly constrained social morphogenesis and chaotic social morphogenesis, there are forms of social morphogenesis guided by modalities centred on managing relations, and networks of relations, with a certain intentionality and symbolic purpose that generates norms and means along the way: this is guided or steered social morphogenesis. It generates new social forms on the basis of their own guiding distinctions. These distinctions, however, do not act as constraints on the arena of opportunities but serve, rather, to broaden opportunities by modifying existing constraints.

Obviously, constraints are not easily changed, but the agents/actors intend to achieve this by generating a new relational order through steering their interactions in the intermediate $\mathrm{T} 2-\mathrm{T} 3$ phase, in order to change the constraints posed by the conditioning structure. If we understand constraints as the initial system's guiding distinctions, steered social morphogenesis aims to alter such criteria. For example, it changes the freedom-equality pair to the equality-solidarity pair, or it moves from the binary constraint of freedom-equality to the ternary constraint of freedom-equality-solidarity. There are many social practices moving in this direction. Examples of the first kind are all those procedures which are based upon "concertation" as 
a method of conflict resolution. ${ }^{10}$ Examples of the second kind are all those social policies which provide welfare entitlements on the basis of the fact that the individual accepts certain conditions for performing acts of solidarity towards other individuals or the community. In the fiscal field, many incentives to economic actors are given on the condition of acting in a manner which is supportive to weak people or particular disadvantaged social groups.

The operations proper to a steered social morphogenesis are modes of connecting fundamental principles governing the processes rather than opposing them with binary thinking. Changes of social forms are not conceived as an adaptation to an environment that is evolving and imposing choices that alter relations' primal way of being (their sui generis nature), but instead as an empowerment of the potential that the relationships possess in themselves, with their possible variations. The steering operations are carried out by continuously reorienting the relationships towards the purpose to which they must aim, taking into account the necessary variations in the means and norms. This is possible if agents return (reflexively) to the roots (value and objectives) of what constitutes and characterises the target relationship as a new social molecule. The generation of new social forms, including transformations of personal and collective identities, is rooted in the goal sustained by the latent values that characterise, distinguish, and constitute that social form - which is not reducible to the whims of the actors/agents.

The distinction among the three types of social morphogenesis consists precisely in the way social relations are conceived and treated. Highly constrained social morphogenesis conceives of social relations as transactions emerging from individual choices made in an arena of opportunities that is strongly delimited by existing social structures. Liquid social morphogenesis is a-relational, that is, it does not attribute any structure to the relation as such. Steered social morphogenesis operates with a relational code that confers creativity to the structure of the social relation, which can modify the existing reality by generating a new relational structure.

\section{Summary}

Modernity opened Pandora's box of social relations, taking them from the world of the probable to the world of the possible "always otherwise"

10 The word concertation is used especially in European politics to define a modality of governance. It means cooperation, as among opposing factions (for instance trade unions, corporations, and government), aimed at effecting a unified proposal or concerted action. Each actor accepts limitations on its freedom of action in order to give priority to a solidary solution. 
(always possible in another way). Social realm now sees relationships of all kinds arise, where nature and culture continually fight and recombine. The tension between the two poles grows without breaks. Precisely this tension between nature and the artificial world, far from erasing nature, is today generating a new social relationality.

There are those who think that this relationality can be a "rational reconstruction" carried out under the aegis of rational choice (Coleman 1993) and others who think in terms of self-organising complex systems (Günther 1976). In my opinion, these approaches can easily end up in a form of upward or downward conflation, respectively. Instead, a progressive social morphogenesis seems to require a kind of relationality that responds to what I call "relational reason" (Donati 2009).

Unlike modern rationality, the relational reason seeks out significant social relations and not objects (the fetish of commodities). When the question of meaning that is inherent in the social relation does not find an answer in what is offered by nature (things and human persons), it must necessarily build a social world that does not exist in nature. Human nature thus comes to be subjected to the tension of the artificial (the so-called post-human, hyper-human, cyborg). If the artificial does not take into account the natural needs of human relationality, it generates the non-human. This is demonstrated by the cases of communication technologies that produce psychic and social pathologies because they do not take into account the needs of human sociability.

In this tension between the relational nature of human beings and a purely rational or functional construction of society that disregards the former, spaces open up from which the relational forms that characterise the social morphogenesis of contemporary society are generated.

Compared to the past, the qualitative difference of today's social morphogenesis is found in this: modernity says "I" and opposes it to "You" (including the "You" of nature); the aftermodern says "We" and therefore seeks a relationality that creates synergy between what is natural and what is artificial, but it can realise relational goods only by overcoming the lack of relational culture of modernity. The new digital technologies can help create new "communities" (We-relations), but they also present strong risks as we will see in Chapter 9.

As Marx and Simmel foresaw, capitalist society was bound to generate more and more autonomous social relations that bent back upon the agents to condition their deeds (and the natural world) profoundly. The society of the future will be able to escape a chaotic social morphogenesis only if it succeeds in inflecting morphogenesis in terms of a "We-in-relation" (a relational We) - in other words, if society will be able to sustain social morphogenesis through relational subjects. 


\section{The morphogenesis of social morality}

\section{Where the agency/structure debate has failed}

What is it that comes to be socially defined as "morally good" or "bad"? Or simply acceptable or inacceptable? And by whom? Who defines which moral norms are accepted and which are excluded?

For many sociologists, social morality consists in the attribution of meaning to the action taken by individual humans who, in aggregating with one another, arrive at a consensus that constitutes collective morality. The latter is then reflected back to single individuals, directing their actions towards a social order in which the moral distinction between good and bad corresponds to that between normality and deviance.

What I want to emphasise is that, in the mainstream view of social morality, the circularity between agency and (cultural and social) structure glosses over two problems: (i) the issue of the relational constitution of agency/Self and (ii) the issue of the relational constitution of cultural and social structures.

In my opinion, much of the debate over agency and structure is flawed by these ways of reducing morality to a game between individuals and social structures. What they seriously undervalue and distort is the theme of morality as a relational phenomenon. The morality of social relations is attributed to the individuals as such, who feel uncertain, anxious, insecure. The relatively autonomous role of social relations in forging morality is overlooked or relegated to the shadows.

My intention is to understand the origin and change of morality, not as the result of aggregations of individuals in a context defined by certain (social, cultural, and communicative) structures or, vice versa, as the influence of structures on individuals' agency, but as the product of the relationality that develops in networks of social relations that connect social agents/actors.

The problem needs to be brought into focus. It is a matter of understanding if - and if so, how - one can speak of an intervening variable, that is the morality of social relations as such, which constitutes a sui generis order of reality in as much as relations have simultaneously to meet individuals' needs, on the one hand, and the needs of the governability of institutions, on the other. 
The question is, does a morality of networks of relations exist that is distinct from the morality of individuals and from that of sociocultural systems? Put another way, can we speak of morally good social relations/ networks and morally bad social relations/networks? Can we do this without falling into the holistic fallacy of believing that social relations/networks can themselves think (morally)?

In my view, the morality of a social relation (or network of relations) is due to the fact that it can produce relational goods or relational evils. In order to produce relational goods, a social relation/network should meet the following requisites:

1 Be an emergent phenomenon.

2 Be morally good in itself and not merely a matter of subjectively being deemed so.

3 Be such as to give the subjects individual goods that they could not obtain otherwise.

Love, hate, forgiveness, and friendship are social relations - and not only individual feelings - that are morally good or bad as feelings. Contempt, the refusal to help, aggression, and violence are morally bad social relations, and not only individual feelings. Certainly, the moral qualities of social relations have correspondences in the properties of individuals, but they are not the same thing because these are two different orders of reality. The morality of reciprocity on the part of the individual (as a feeling, attitude, or act) is not the same thing as the morality inherent in the social relation of reciprocity. I will now try to explain these assertions.

\section{An alternative view}

In my view, morality can be analysed from three points of view, which correspond to three different paths for the formation of moral agency.

Path [1] explains social morality by the conditioning of social and cultural systems which, through their normativity, impose themselves on individuals' agency. Here are situated theories of the "collective conscience" (e.g. Durkheim and Mary Douglas) and of cultural traditions (e.g. Putnam and Fukuyama). The morphostasis/morphogenesis of morality is explained there as the product of normative systems that reproduce themselves or transform themselves on the basis of a logic that is inherent in the same social and cultural institutions, in functional and/or communicative terms. As Mary Douglas (1986) would say, morphogenesis happens when institutions, which are supposed to be thinking entities, change their way of thinking. As Luhmann would have it, the morphogenesis of moral norms is the product of the change of semantic codes due to the effects of reflective mechanisms of communication. He assumes that, in a funtionally differentiated society, the process of communication depends on the inherent laws of the 
communication system itself, which is ironically expressed in the aphorism, "Only communication can communicate." Morality is defined as a special form of communication that carries with it only indications of approval or disapproval. "The moral is not something good. Of course, that should not lead us to say that the moral is something bad [...] The moral functions only as a distinction" (Luhmann 1993: 996). Trust itself, which for Parsons arises from the normative order of the open society, in Luhmann can no longer count on norms that disappear and must be entrusted to communication (Jalava 2003). But, I observe, this does not mean that there are no norms: the point is that the norms of a presupposed moral tradition are replaced by the norms of communication, that is by the mechanisms of codification.

Path [2] is opposite and complementary to the first one. It explains social morality as the product of individual actions that generate a collective system of values and norms by aggregation. A number of individuals, driven by mechanisms of an aggregative type, participate in processes of identification with a shared set of symbolic and normative values that replace other, existing values. Here are situated the collective movement theories. Examples are radical feminist movements, antiglobal, LGBT, protest, and revolutionary movements, such as the so-called Arab Spring. The collective movement replaces certain values and moral norms with others. It is a matter of understanding what this path's specific morphostatic/morphogenetic process is. In general, it consists in the fact that, given certain forms of systemic conditioning, large groups (or even masses) of individuals set themselves up in reaction to them and radicalise certain values: these groups can be of a conservative type (e.g. fundamentalist religious or racial movements) or of a transgressive or rebellious type (e.g. radical social movements on gender issues or for the abolition of private property).

As I have already said, the majority of theories explain social morality as the product of a combination between these two paths. The most emblematic example is the morality that I call lib/lab. This consists in the fact that, on the one hand, the morality of individual freedom and of free aggregations of individuals (lib morality) is invoked while, on the other hand, morality is instead considered as an institutionalised entity that has the task of guaranteeing equality of opportunities in the relationships between individuals (lab morality). It is assumed that (i) on the one hand, free individuals can adhere (or not) to a shared system of values and moral norms (the lib dimension of moral agency), and (ii) on the other hand, the system of shared moral values works in such a way as to guarantee its own effective realisation through system mechanisms of a "mechanical" type (the lab dimension of moral agency), which end up strengthening individual orientations. Morphogenesis happens only if aggregations of free individuals change the moral system; otherwise, the system continues to be reproductive.

A macroscopic example of the lib/lab view is the idea of the European Union's master plan as supported by the former President of the European Commission, Romano Prodi (2002: 20): "The integration of free market 
forces and competition, on the one hand, with equality of opportunity for all citizens, on the other, is the master plan of the new European construction." This is a vision of society that has its roots in early modernity and, to a certain extent, at the end of the 20th century has found expression in Giddens' (1998) so-called Third Way. It was relaunched by Tony Blair in his speech to the British Parliament in July, 2014, when he proposed the copenetration of state and the market because neither of them can resolve social problems by itself. Lib/lab theorists care little that actual reality demonstrates that this model produces more problems than it solves. They do not see that its failures are due to the fact that it forces social subjects to act within the double bind between state and market.

In my approach, there is also a rather more complex path. In this path [3], morality certainly depends on moral (primary and corporate) agents, but it has its own relational dynamic. If we agree that individuals necessarily find themselves confronting already existing institutional structures, the morphogenetic process starts from individuals who create networks of social relations which, on certain conditions, confirm or alter moral institutions (social and cultural systems). Institutions, in turn, and always on certain conditions, strengthen or alter the morality of networks of social relations and, thus, influence individuals. In this path, the explanation of morality must come to terms with what happens in the intermediate space between individuals and institutions (normative systems). The ways in which (intersubjective and/or impersonal) social relations structure social networks become crucial. The understanding of the social morphogenesis of morality becomes much more problematic because the intermediate transitions are subject to great variability.

Figure 5.1 offers a representation of these processes. The graph contains two circles which are connected by an arrow going from the first to the second and by an arrow going from the second circle to the first. System morality is placed in the first circle, agency morality in the second. One affects the other. In the middle between the two circles there is a rectangle which indicates the existence of a network of relationships that connects the two previous circles and represents the morality of the social network in which the actors are embedded. Mutual input and output lines are drawn between this central rectangle and the two circles. These arrows indicate that the social network acts as an intermediary in the dialogue between system and individual morality.

What I want to elucidate is the difference between the morphogenesis described through paths [1] and [2], which correspond to the morality of lib/ $l a b$ arrangements, and the morphogenesis that passes through social networks (path [3]), which corresponds to new relational configurations.

In the circular path between [1] and [2], what happens in the intermediary temporal phase (T2-T3) of the morphostatic/morphogenetic process is regarded as an aggregation of individual behaviours that identify themselves - positively or negatively (through positive or negative 


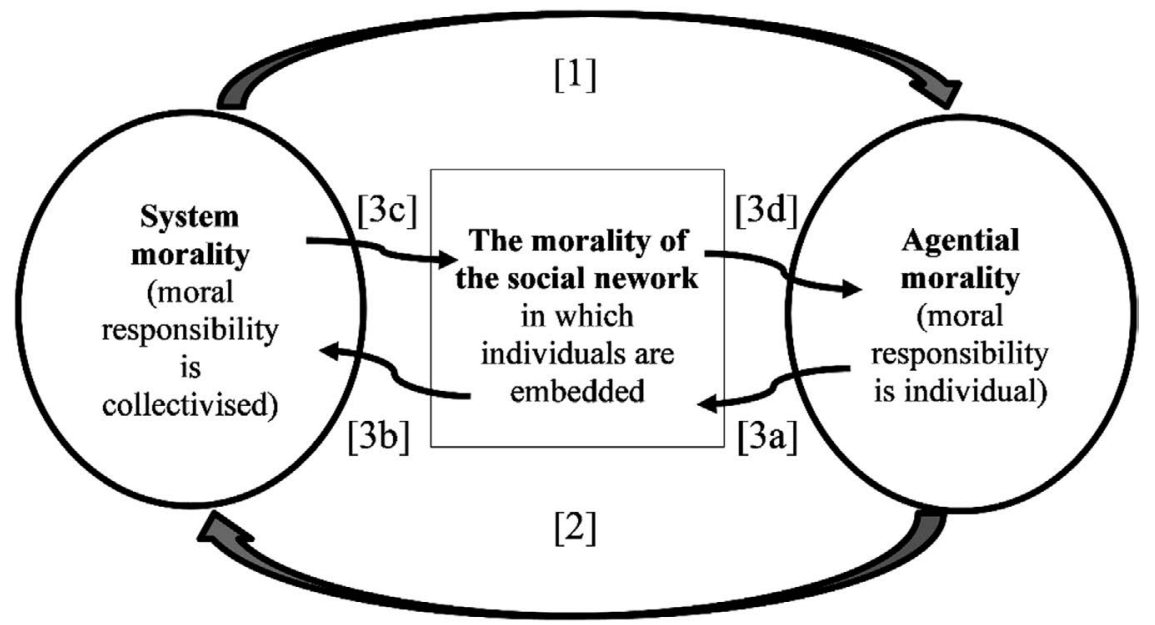

Figure 5.1 How system morality and agential morality are mediated by the morality of social networks

Source: Author.

feedbacks) - with the same symbols/values that, if they are institutionalised, reflect on the aggregations of individuals and can be confirmed (morphostasis) or altered (morphogenesis) by new aggregations of individuals. The keystone of this interpretation of social morality lies in individual agency's adhering to one or another "group consciousness" (corporate actors), which can be of the majority or specific to minority groups (as is called for by the doctrine of multiculturalism). According to this view, the participation of individuals happens through their identification with a shared symbolic "focal point" (a social class, a cultural or religious tradition, or a social movement) that does not necessarily require specific interpersonal and network relations among the participants.

In path [3], instead, we can observe changes of morality in much more detail through different moments because in phase T2-T3 operates a relational logic which is overlooked or underestimated by explanations based on the paths $\{[1]-[2]\}$ (Figure 5.1).

\section{The obsolescence of classic paradigms}

The lib/lab paradigms of social morphostasis/morphogenesis can generally be traced back to a framework in which the conditioning structure at the initial time T1 dictates the moral norms with respect to which individuals must take a position in their interactions in phase T2-T3. This happens whether the conditioning structure privileges moral norms of a libertarian and individualistic type (as happens in the United States since the lib side prevails over the lab side there), or whether the opposite happens, as 
in Europe, where the lab side of political regulation prevails over the lib side of the free market. In this paradigm, it is claimed that individuals can conform, or not, to the conditioning structure according to modalities that Merton (1938) synthesised in his famous five types of adaptation to legitimate ends and means (among which are moral norms) that characterise the conditioning structure.

Merton's typology is built on positive and negative feedbacks on the part of individuals vis-à-vis the conditioning structure's ends and norms. These are first-order feedbacks. The scheme assumes that the formation of collective actors in phase T2-T3 consists in the adherence of a certain number of individuals to each of these modalities (this is an example of the use of the binary agency/structure, micro/macro scheme). The aim of this theory is to discover how some social structures exert a definite pressure upon certain persons in the society to engage in nonconformist rather than conformist conduct. Merton's answer is that "certain phases of social structure generate circumstances in which infringement of social codes constitutes a "normal' response" (Merton 1938: 672).

With reference to change in morality, it is assumed,

1 conformity and ritualism produce morphostasis,

2 renunciation leads one to stay out of the social arena, while

3 innovation, in as much as it alters norms but not ends, seems to constitute the modality of internal development of the same system according to the Parsonian model of modernisation (the Parsonian model of up-grading modernity and successful modernity).

Where is morphogenesis? My thesis is that the Mertonian type of scheme commits a series of fallacies. The first fallacy is that it explains morphogenesis through positive and negative feedbacks alone. The second fallacy, in parallel, is to assume that morphogenesis is produced by automatic mechanisms. For example, some sociologists assume that buying newspapers, donating blood, or participating in sport or cultural associations automatically indicates that these individuals share a civic culture and have relations of trust, cooperation, and reciprocity with one another. Such a correlation, much less a causal connection, does not have any empirical foundation.

In other words, these theories overlook the feedbacks activated both by individuals and institutions on social relations, and they think that adherence to certain norms produces social change in and of itself. To my way of thinking, it is not so. Adherence alone to the change of a moral norm (via positive feedback) is not sufficient for explaining the changes in the relations that individuals have with one another and with the object of their actions. If we want to understand the current morphogenesis of morality, it is necessary to introduce a relational view on the ways in which moral norms form and change society. 


\section{Why relationality matters in generating different morals}

Feedbacks to social relations follow a different logic compared to the logic inherent in feedbacks to single actions. My argument is that feedbacks to social relations (relational feedbacks), rather than feedbacks to simple actions, change the normativity of the generative mechanisms that produce social morphogenesis through positive and negative feedbacks.

The argument's key point is that reactions (feedbacks) to the single actions of Ego and Alter and reactions to the relation between them are different orders of reality. It is possible that Ego rejects (or accepts) Alter's action and accepts (or rejects) the relationship with her/him, and vice versa, if and only if the feedback exercised towards the single action is of a different order of reality with respect to the feedback towards the reciprocal relationship. The different order of reality implies a different normativity (logical rules) in as much as agents refer to single actions or, instead, to social relationships.

Let me synthesise the differences between conceiving $\mathrm{M} / \mathrm{M}$ processes on the basis of mechanical mechanisms (MecMec) and on the basis of relational mechanisms (RelMec). In the case of MecMec, moral norms arise from the adherence/deviation of individuals' actions vis-à-vis system morality. In the case of RelMec, moral norms arise as a consequence of taking into account the autonomous relationality between agents. Let us look at this in more detail.

One, in the case of MecMec, moral norms are expected to operate independently of the social interrelations among actors, and therefore,

- The normative order depends on conformity/deviance of individuals' actions towards the system (this is the logic of the "agency and structure" debate).

- The feedbacks can be positive and negative, but not relational.

- The black box of the mechanism works with a trivial causality (operating with internal functional constraints and given boundary conditions).

- The normativity of social mechanisms is supposed to be "automatic" in so far as the interactions in the mediating social network (phase T2-T3 of the morphogenetic process) are bound by standard norms.

- The outcomes are aggregative social phenomena or functional performances, that is the outcome is determined by the mechanisms inherent in the social network conforming to structural constraints.

- As examples we can think of social systems/institutions working as goal-seeking machines (e.g. traffic norms); organisations relying upon belief-formation mechanisms, bandwagon effects, snowball effects, etc. (e.g. advertising agencies or election campaigns); procedural justice; procedural democracy; adiaphoric normativity in facing social issues; bureaucratic anormative regulations. 
Two, in the case of RelMec, norms are expected to emerge and operate through the dynamics of sociability/relationality among agents, and therefore,

- The normative order depends on social relations generated by agents and their networks.

- The feedbacks are positive and negative at the first order, and relational at the second order of the interactions in the network.

- The black box of the mechanism works with a non-trivial causality (whose workings depend on internal complexity and in interaction with the environment).

- The normativity of the social mechanisms is sensitive to the interactions in the mediating social network (phase T2-T3 of the morphogenetic process) and, for that reason, is nomos-building.

- The outcomes consist in the emergence of relational subjects (primary and corporate agents) whose normativity is an expression of an underlying interacting social network.

- As examples we can think of relational couples (e.g. the higher the commonality of the couple's external network, the greater is the sharing of conjugal roles); civic associations; community foundations; social streets; peer production and co-production; relational goods; "Beyond GDP" indicators (where economic well-being depends on the quantity and quality of bonding and bridging social capital - see Stiglitz Report 2009); justice based upon norms of social (relational) equity; fair trade; deliberative democracy; associative democracy.

RelMec are generative in as much as they are relational operations. They are so when the normative dimension of the mechanism corresponds to a "logic" (in the sense of a rule or set of rules) that combines the elements of the relationship between the agents (Ego and Alter) in such a way as to ensure some validity and efficacy in what emerges from their relationship. Otherwise, it is a MecMec.

For example, the basic mechanism of Western political democracy is the rule that after a contest between the majority and minority, one votes yes or no on a proposal. This logic (normativity) is not relational. The morality is inherent in the logic of numbers: the political decision is taken on the basis of the majority of votes. This is the moral norm leading to political decisions. In this case, voters vote through the mechanism of positive and negative feedbacks.

The activation of RelMec does not happen in the current political system of parties because they operate with a binary morality that does not allow consensus building to happen through the establishment of networks of relations among the decision makers who are members of the different political parties. If this were possible, it would also be possible to form democratic networks of decision makers in political bodies that reflect the networks of civil society. 
This possibility is available in the decision-making sphere of certain third sector organisations (such as in true social cooperatives) in so far as they give priority to the value of the relations of civil society, which their decisions create. It could also happen in the political system if a "relational state" model was adopted, which would operate through relational inclusion rather than through a compromise between market and state, that is lib/lab inclusion. "Relational inclusion" means that a full participation of people in society (or a social subsystem) is realised by providing citizens with the opportunities to act as relational subjects in relation to other social subjects - with the same rights and obligations - and not on the ground of adhering to market competition ruled by the political power. The lib/lab system is built precisely in such a way as to make the system immune to this network of social relations.

Human social relations exist at a sui generis level of reality. Many examples can be given to show that this autonomous level of reality exists.

First example, reciprocating a gift cannot be explained by an automatic functional mechanism, nor as a mere individual response that Ego gives to Alter, who gave Ego the gift. ${ }^{1}$ Reciprocation is a relation that has its own morality; it is the expression of a moral norm that does not depend either on the impersonal function of the gift or on the individual action of giving "something" that is owed to one person in particular, but resides in the suprafunctional meaning of the true gift relation as such, as a good in itself (which means for the goods it carries with it beyond the utility of a particular exchange of things and/or services).

Second example, the electoral vote follows a different moral norm (logic) if it is given as a relation to a person or to an ideology in the contest between political parties. The reason resides in the fact that the morality of interpersonal relations is different from the morality that is applied to impersonal relations.

Third example, in a hierarchical network where the founding relation stipulates obedience, the ordinary dynamics are mostly productive of conformity to norms, while a network that works with a "be different" norm is more likely to produce anomie (think of that large part of consumer advertising, aimed at teenagers and young people, which carries the motto "be different"). An example of conformity is Horstink's (2011) research on a group of soldiers in the military. This study investigated the influence of relationship density and relationship power on moral reasoning. Findings showed that social network properties influence moral reasoning by creating similarity within groups. An example of the latter type is provided by studies on those social networks on the Internet that aggregate individuals who act as hackers

1 The idea that moral responsibility consists in the infinite debt of whoever has received the gift, as Bauman (1998) maintains, is clearly individualistic. Perhaps it is because of this that Bauman considers morality as a poisoned gift (Junge 2001). 
without having anything or very little in common (Haythornthwaite 2002, 2005). In both cases, a crucial explanatory factor of the different outcomes lies in the properties and powers inherent in the specific relational morality of the networks.

In all of these examples, the morality of agency follows one "logic" rather than another because the reference to the relations entailed by the agency (i.e. the moral good or bad inherent in the relation) changes. If the relations are virtual, the morality also can become "virtual," meaning that the moral norms lose some of the constraints characterising and binding interpersonal relationships (it is empirically verified that, in the environment of virtual communications on the Internet, users are often encouraged to simulate or suspend certain rules of responsibility that they cannot avoid in real relationships). By changing reference to different kinds of relationality, morality can follow a different logic (e.g. in the anonymous matrix of communication provided by the Internet, morality becomes more impersonal, and, by virtue of the means of communication, the rules combining goals and value-patterns may disregard the distinction good/bad which would be necessary in a non-virtual environment). On the empirical plane, we see that the normativity of being "ethical" towards individual persons or impersonal institutions (impersonal discourses, systems, distant others) is actually different precisely because of the different morality (tighter or looser, more expressive or more utilitarian, etc.) implied in these relational references.

It is for this reason that it is necessary to take into account unexpected, indirect, and perverse effects. The following seems to me to be an interesting example. If we accept that in late modernity the sociocultural structure imposes the moral imperative to "be different" (i.e. "choose your own option of what is good and bad"), we can ask, why is moral indifference the unexpected and unintentional outcome? A possible explanation in terms of the morphostasis/morphogenesis scheme is the following. The morality of institutions pushes towards moral individualism, but the network of individuals has a structure and moral dynamic that are such as to nullify the value of personal choices. The network's moral reflexivity is hindered or fractured (as is the postmodern public sphere in general), so that indifference (non-differentiation) predominates in moral choices. This process is obviously favoured by those ICTs which work by cancelling out the value of moral differences due to the composition effect (choices elide with one another), so that the medium becomes the moral norm ("the medium is the message").

\section{The morality of welfare and its morphogenesis}

From its inception, the welfare state has been the object of endless discussions regarding its moral foundations and its consequences for the morality of society as a whole. It is not possible to discuss this issue extensively. I will come to the point. 
The point is that over the course of its development, the welfare state has adopted an increasingly impersonal morality. The moral responsibility for social problems (poverty, unemployment, health needs, pensions, social assistance, as well as education and housing, etc.) was handed over to an impersonal "system." The welfare package (inclusive of all basic needs that must be satisfied to have a decent standard of living) has become an automatic social right (a right of citizenship), in the sense that it does not take into consideration the beneficiaries' conduct. The morality of this welfare resides in the principle of solidarity (i.e. of inclusion and social cohesion) realised through state redistribution.

With reference to Figure 5.1, we can say that moral responsibility passed from individuals to the system (from [1] to [2]), completely skipping over social networks (path [3/a, b, c, d]). The system has thus proceeded to protect single individuals, relieving the social networks (families, informal networks, associations, and institutions of civil society) of obligations towards the collective well-being.

In recent decades, a vast literature has shown the negative effects that this course of action has had on the forms of primary solidarity (informal networks) and secondary solidarity (networks organised in associative forms). The outcome of this story has been, and is increasingly, the crisis of the welfare state, which derives from not only the State's inability to address social problems (the fiscal crisis, the inadequacy of public bureaucracies, etc.), but also, and especially to the fact that it generates perverse effects, among which are the progressive lack of responsibility of individuals and the fragmentation of social integration. Undoubtedly, the rhetoric of conservatives and parties on the right when they speak about this issue is overblown. Nevertheless, beyond the rhetoric of conservatives, the problem is real.

The morphogenesis of the moral norms that justify the welfare state is thus in a radical crisis. My thesis is that the morphogenetic process of the moral norms that must give a moral meaning to welfare is blocked today because the existing model of the welfare state is propped up by the reproduction of lib/lab morality: the lib side calls for a return to individual responsibility (autonomous individual reflexivity) while the $l a b$ side relaunches public intervention with Keynesian types of adjustments, reaffirming the primacy of collective responsibility. The proposal of a "third way" that introduces a new mix between moral lib principles and lab principles (Giddens 1998) has not yielded significant results. Proposals of this type cannot work because lib/lab morality is blocked; it does not produce a creative morphogenesis, but only a bound morphogenesis.

It is here that the argument about the morality of social relations makes its entrance. Individuals must be morally free, and the social system must guarantee social solidarity, but how can we proceed so that by choosing one we do not eliminate the other term?

Figure 5.1 suggests that we look at how individuals and systems interface through social networks: in other words, whether or not they invest 
the relationality of the networks with morality and how they consider the morality of interpersonal and communitarian relations as such. Freedom must make reference to the concrete relations that individuals create and in which they live. And the system must take care of relations by evaluating whether they produce relational goods or evils (this is the "relational state"). The goodness or badness of social relations comes into play, in and of themselves and in their effects. It is here that a new morality of welfare comes into being. Alongside other morphogenetic processes, another type of morphogenesis is taking shape, which I call relational.

On the theoretical plane, the sequence of change that the morality of welfare has undergone can follow different paths of morphogenesis in the cycle T1-T4. I offer three as examples (Figure 5.1).

- T1: the "starting" situation is given by the systemic crisis of the welfare state's morality: the welfare state enters into crisis perhaps because it nullifies individuals' moral responsibility by completely impersonal principles of responsibility, or perhaps because - vice versa - it appeals to individual responsibility while overlooking the norms of solidarity.

- In the intermediate T2-T3 phase: the interactions between agents represent the search for moral norms in response to the systemic crisis; these interactions can follow, roughly, three paths:

i Interactions are guided by an action model that tries to adjust the norms of negotiation between state actors and market actors, leaving aside other actors; this modality (of communicative reflexivity within the lib/lab system) represents a search for internal adaptations to the "system," which are generally of an opportunistic type.

ii Interactions are predicated on action models characterised by an autonomous reflexivity, so that the social networks, lacking any shared moral constraints, become chaotic.

iii Interactions adopt action models supported by a meta-reflexivity that is oriented towards building social networks in which there is an important effect of "open coordination"2 between the parties, valorising the morality of reciprocal relations (relational steering).

2 As an example, we can refer to the open method of coordination (OMC) adopted by the European Union. The OMC provides a new framework for cooperation between the Member States, whose national policies can thus be directed towards certain common objectives. Under this intergovernmental method, the Member States are evaluated by one another (peer pressure), with the Commission's role being limited to surveillance. The European Parliament and the Court of Justice play virtually no part in the OMC process. The OMC takes place in areas which fall within the competence of the Member States, such as employment, social protection, social inclusion, education, youth, and training. 
- T4: the structure of emergent morality depends on how the normativity of phase T2-T3 was elaborated; according to the three above paths and three types of morphogenesis are possible,

i A morphogenesis of lib/lab morality (the lib/lab is not in and of itself morphostatic); the emergent norms are a combination of criteria of efficiency and efficacy in the definition of welfare morality.

ii An unbound morphogenesis of lib/lab morality, which means the appearance of moral norms of individual freedom and of collective constraints that proceed on their own, without significant connections between them, thus producing contradictions and perverse effects.

iii A morphogenesis of morality that amplifies, selects, and stabilises new opportunities on the basis of a normative criterion that valorises relationality between the parties as a primary good; for example, welfare measures come to be considered as morally good/bad depending on whether or not they lead to the flowering of interpersonal relations (in the positive case, one speaks of "relational state," "welfare society," etc.); here the moral norm of social structures is based on the qualities and causal properties of the social relations that are promoted.

The third alternative (relational morphogenesis) indicates a morality characterised by the moral norms of equitable and sustainable welfare as the product of sui generis relational networks. As examples one could cite, co-production (Pestoff 2009, 2015), public-private partnerships generating new networks at the local level (Evers 2015); social cooperatives able to be counter-cyclical instead of having only a buffering role on the impact that the systemic crisis has on citizens (Vidal 2015); and, in general, the so-called relational turn in social innovation (Fløysand and Jakobsen 2011).

Every society has to come to terms with an intrinsic ambivalence: on the one hand, it must make individuals understand that they depend on the collectivity and that, therefore, the altruistic orientation must prevail over an individualistic orientation, but, at the same time, it has to push individuals to maintain a certain distance from the prevalent norms so as always to be able to develop their own evaluations and thus guarantee a certain degree of creativity, innovation, and pursuit of change on the part of the individual.

The problem of lib/lab is that its moral norms do not provide a solution to this ambivalence. We can take the case of bibliometric evaluations of scientific research. Leydesdorff (2014) considered the ethical and cultural consequences of relying on metrics to assess the quality of scholarship, and reached the conclusion that the present system, centred on individual production, penalises the research of groups, networks, and between cooperating individuals. The answer to the problem of how to combine individual creativity and collective subjectivity can only come from a relational 
approach that highlights that innovation is a product of networks that share norms formed in an autonomous manner by the sociability of participants without being imposed by a superordinate structure. The problem is whether the network is able to avoid the absence of or lack of clarity in the norms. The answer is the relational subject, the morality of the We-relation. Lacking this, networks fail.

Let me recapitulate the fundamental argument. The social and cultural structure given at $\mathrm{T} 1$ enters into a systemic crisis as regards the moral norms that it contains, explicitly or implicitly. The crisis leads to the commencement of interplay in phase T2-T3, from which derive effects to which, de facto, society responds, depending on the case in question, with three types of morphogenesis that have three types of outcomes: (i) circularity of the morality between subjective norms and institutional forms (Beck, Bonss, and Lau 2003); (ii) widespread anomie and morality as a reaction to having hit bottom (Teubner 2011); (iii) morality generated through new forms of relational steering (Donati 2015b). This is the moral scenario of society during the transition to aftermodernity.

\section{The societal moral fabric in the era of globalisation}

The scenario that we have before us is that of lib/lab systems continuing to operate while encountering growing failures and perverse effects. Their morality is self-defeating. The rapidity of failures depends on the accelerating erosion of certain values and traditional norms that early modernity had preserved in the past. The immediate consequence of such failures is perceived as unbound morphogenesis, which means growing widespread anomie.

We can hypothesise that morality in the era of digital globalisation will proceed according to three concomitant types of morphogenetic changes typical of each T1-T4 morphogenetic cycle. The processes can be described through the morphogenesis of the basic structure of social relations (see relational-AGIL in Figure 4.2).

At time T1, we find a structure AGIL-1 that contains its own Morality-1, which conditions agents when they begin to interact with one another. At time T4, we end up with a new structure AGIL-2 that characterises Morality-2.

In phase $\mathrm{T} 2-\mathrm{T} 3$, three scenarios are possible:

a Bound morphogenesis: the network of agents operates on the basis of Morality-1, which at time T4 can produce both morphostasis (communicative reflexivity) or a bound morphogenesis (autonomous reflexivity); within the double bind of lib/lab morality, Morality-2 reproduces Morality-1 or is a variation of Morality-1 that maintains the lib/lab morality. 
b Unbound morphogenesis: the network of agents breaks the structure of Morality-1 and at time T4 generates an unbound morphogenesis, that is an anomic morality. Here the Morality-2 is characterised by a relational logic that is unable or refuses to draw distinctions (thought to be discrimination instead of a fair recognition of a difference that must be treated as such, because justice requires that different realities are not treated uniformly, but in an equitable way) and, thus, does not valorise connections, but fosters reflexivity in a fragmented or impeded way.

c Relational morphogenesis: the network of agents operates with strategies of a relational steering type and produces relational morphogenesis. The structure of AGIL-2 is characterised by a meta-reflexive logic that indicates ends and means as a function of relations' value. This is the case of morality that is generated in those social spheres where relationality is taken seriously as a source of moral norms. In other words, interactions are guided by the morality of the relation as such, which is based on relational feedbacks. New opportunities are created by means of relational steering: the network, in interaction with the exterior, introduces variations in resources, actors, power relations, and norms that legitimate the variations that stabilise relational feedbacks.

This framework underscores the fact that the structure of the relation that establishes and characterises the social network contains the moral norm that organises the means and ends of agency - both individual and collective - as a function of the value of the relation itself.

In case (Morality-2 a), a norm of collective utility prevails that maximises the criteria of efficiency and efficacy. An example is found in organisations that operate with a managerial style (business morality).

In case (Morality-2 b), a norm that maximises criteria of contingent preference prevails. It could be of an expressive or opportunistic type, in the sense of seizing the most attractive opportunities of the moment: for example, the morality of Facebook networks.

In case (Morality-2 c), a norm prevails that gives priority to the criterion of the moral value (the dignity) of the relation that characterises the social network. Civic networks, such as "social streets" (an initiative born in Italy and now widespread in many countries: see on Google), are an example of this type if and when the relation that inspires associative agency is the value to be pursued, independently of its utility, efficacy, or efficiency. In these social practices, what matters is not the material goal that can be achieved (for instance, the maintenance of a common good like a public garden in the neighbourhood) as such, but "the sociality behind it," that is the sociality among the neighbours who produce it.

The third solution at time T4 (Morality-2 c) entails the primacy of the moral value over the relation's other components. Moreh (1986) has shown that the economic theory of utility does not take into account moral 
behaviour, that is behaviour in which an individual faced with two courses of action may choose the one that is less profitable to him or her. According to relational theory, when the goals that the individual has to achieve consist of peculiar social relations (e.g. personal care, education, friendship, etc.) then the morphogenesis has to be steered towards the achievement of higher relational goods. My thesis is that this behaviour has greater probabilities of being chosen when the individual is in a relational context endowed with certain properties and powers rather than in another one. The context is not only a constraint, but also a resource, a pool of opportunities offered by the network formed with others.

Luhmann (2008b) wonders, "Are there indispensable norms in our society?" His answer is negative. In his view, it is impossible reasonably to expect that any given moral or legal norm is normatively indispensable. This answer is in line with the ontological and epistemological premises of his approach, which is decidedly sceptical and amoral. I think that he should be challenged with an even more paradoxical question: "Are there still indispensable social relations in our society?" The latter issue is strictly linked to the former. Let us take the example of work relations. No doubt work is increasingly considered an indispensable social relation, not only from the standpoint of economic support, but also of the person's emancipation/humanisation, and in this sense, it is a moral imperative, which of course admits of exemptions in special circumstances (such as chronic or severe illness).

To understand the argument according to which there are social relations that are morally indispensable, it is necessary to consider the fact that social relations always involve morality. The practical moral norm (not the ethical principle, which is located on another level of reality, that is in the cultural system) is redefined and changes the moment that the agent reflects on relations with others in a context, and not when he/she reflects internally on his/ her own "I." The individual's internal reflexivity considers the moral norm in order to define his/her moral agency in terms of the aspect of his/her subjective determinations (the process of discernment, deliberation, dedication is analysed in depth by Archer 2003), but the moral norm has a dynamic that does not depend only on individual agency, whether of a single individual or of a multiplicity of $\mathrm{N}$ individuals (aggregated or interacting with one another) - just as it does not depend entirely on the dynamic of structures (social and cultural institutions). The practical moral norm is formed and created in the relational process among agents. The problem is how we can analyse the contribution given to the formation of the moral norm inherent in the social relation (e.g. that of work and family) by, respectively, the single agents, institutions, and the relation itself.

The present moral imperative in Western culture, which has been absorbed into social theory, is that individuals should be active, intentional, autonomous, independent, reflexive, capable, and constantly able and willing to make rational choices in their lives. Behind this moral imperative for 
agency, other aspects of what it is to be human remain in the shadows. In terms of actions, the human aspects consist in the fact that agents "always and as such have patients, beings which the action affects" (Reader 2007: 588). Whoever teaches influences the student necessarily, but the student retroacts upon the teacher. Whoever sells necessarily conditions the buyer, but is also conditioned by him/her. Whoever cares for a sick person has an impact on him/her, but is also influenced by him/her (Hałas 2021). To put this more sociologically, every action entails a relationship. No single person is ever completely an agent or a patient during any one moment of interaction. The Self is called, like it or not, to acknowledge itself as a relational subject. This reality is grasped if one understands that, from the moral point of view, what is at stake resides in the relation; indeed, what is at stake is the relation itself, considered for its moral properties and powers, because it is in the relation that what is good/bad, right/wrong is defined in (and is relevant to) the identity of being an actor or a patient, an active or a passive subject. When the relation presents itself as ambivalent, the ambivalence can be dissolved by determining what type and degree of reciprocity exists between Ego and Alter, given the fact that it is the properties and causal powers of reciprocity that define what is good or bad.

\section{Summary}

Morality is a social fact, but it is treated as being a problem of the individual or the system, or a mix of the two. Current mainstream sociologies explain changes of morality in terms of opposing accounts. For some, morality evolves by systemic emergence. For others, morality evolves through an emergence of subjectivity. If, until a few years ago, these two versions were seen as antithetical (Durkheim's collective conscience vs Weber's individual subjectivity, in particular "charisma"), today structure and agency seem to operate in a synergic manner. My explanation is that this happens because a lib/lab configuration of morality prevails, which intrinsically contains a double bind (the double bind of the system constraint on individuals to be morally "free": see Bateson 1972: 271-278).

a The theses arguing for morality as "systemic emergence" maintain that morality becomes a product of functional systems that, in as much as they proceed only through communications and are (ontologically) only communication, de-normativise society, including human beings, in a system that includes animals, plants, and every other entity, obviously including the entire artificial world of technology.

This thesis claims that in the differential and complex modern societies, the true protagonists of such events and processes are no longer humans or groups of humans with their material needs and their "values," but roles and functions, systems and environments: a whole world of givenness and 
system relations in which human individuals operate like mere interchangeable and perfectly fungible elements. According to Luhmann (2008a), this fact leads to a new style of morality that is founded on the shared interest in reducing fear and no longer on norms.

It is in this way that the era of globalisation presents itself, according to some, as morally adiaphoric. Decisions that need to be taken can no longer and should no longer be regulated based on a substantive distinction between good/bad that appeals to some "nature," "reality," or "objectivity" because decisions on good and bad become "technical," ethically indifferent, adiaphoric.

In this chapter, I have tried to show that this vision commits a macroscopic error of perspective because it does not see the human character of the social as a nomos-building activity. The type of morphogenesis that it recognises is only that of the dehumanisation of social morality.

b In contrast, the theses arguing that morality is becoming completely subjective (morality as "emergence of subjectivity") commit an error of perspective that is symmetrically opposed to the previous one because they make the morality of institutions (structures) coincide with that of individuals. Those who support this thesis (including many academic scholars and even many magistrates in courts) argue that morphogenesis takes place through those cultural movements that claim that people, as pure individuals, have the right to define their identities and their relations as they like. The distinction between social relations (as structures) and subjective consciousness is no longer productive. What individualisation of social relations essentially means is that the perceived relation is the social structure, and that, consequently, both the individual perception and the social structure might vary at people's will. In their opinion culture becomes an experiment whose aim is to discover how we can live together as equal but different; the aim of normativity is less and less to prescribe a certain way of living and more and more to clear the institutional conditions for a multiplicity of lifestyles to be recognised. This means that any collectively shared definition of relationships and individual positions has gone.

My opinion is that these two broad currents are, at the same time, antithetical and complementary (the moral norm refers to two polarities that are simultaneously opposite and complementary) because they entrust impersonal morality to the system and locate the morality of free individuals in the system's environment where it can fluctuate at will. System morality guarantees subjective morality, which can create the most disparate social forms. On the basis of this perspective, the morphogenesis of morality would be destined to play out this game between individuals and the social system.

In this chapter, I have tried to show that this scenario identifies the processes of morphogenesis inside lib/lab that leads to the dissolution of 
lib/lab. If we manage to see that morphogenesis happens through complex social networks, in which specifically human relationality takes on increasing importance - which is different from that of animals and other living beings - we can glimpse other forms of morphogenesis. These forms are not simply the crisis of morality tout court, or the replacement of modern morality by the synergy between system amorality and subjective amorality, but rather the birth of a new morality that valorises the good/bad of social relations upon which human flourishing depends, as well as the morality of human persons.

Once upon a time, society was believed to rest on a morality. When a foundation could no longer be found in religious beliefs, it had to be sought either through reinterpreting these beliefs or looking elsewhere. Contemporary society does not prevent morality from still existing. Rather, it renders morality morphogenetic by continually generating and destroying moral norms through the reduction of what is moral to pure communication, understood as the transfer of information and images. Moral norms seem to become purely communicative. This kind of morphogenesis configures a society riddled with paradoxes. There are those who play on these tendencies to gain advantages. Others become their victims. Still others react, creating other ways of making society, and are carriers of a new morality. In any case, in the long run, the key to solving the problem lies in the fact that a sustainable morality must come to terms with the normativity that is intrinsic in the properties and causal powers of each kind of social relation and, specifically, of the "relational pattern" that characterises the social network in which individuals define their personal identity at a given time among the manifold networks to which they choose to belong. 


\section{Morality and social networks}

\section{Who or what is responsible for the market outcomes?}

Since the time of Aristotle, traditional ethics has imputed the morality of action to the "acting subject" on the basis of a principle of linear causality. Over time and, in particular, with the advent of the modern social sciences, the social, economic, and cultural conditioning weighing on the subject have been evidenced. The result has been to attribute moral responsibility to a "conditioned subject," one that is constrained by existing societal structures (including so-called unjust laws).

Who does bear the moral responsibility for the fact that broad strata of society do not have equal life opportunities as compared to those who enjoy more favourable positions? It is easy to impute the cause of this social fact to societal structures that were produced in the past, for which no one in the present is responsible. Yet the fact is that structures do not think or act by themselves. They are not imposed on the human mind, as some assert (e.g. Mary Douglas). Structures' causality is always mediated by agency. Nevertheless, structures do count for at least two broad orders of reasons: (i) because they define the field of immediately accessible opportunities; (ii) because they place constraints on agents in terms of incentives and sanctions.

This is our problem: given that the responsibility of agents is proportional to their freedom, it is a matter of understanding what freedom agents enjoy vis-à-vis market structures in order to prevent the harm that their actions bring to bear on distant others as a result of the causal mechanisms inherent in the market.

Who is responsible for the harm that one or more market agents/actors ${ }^{1}$ bring to bear on distant others in an impersonal and unintentional way?

1 With the term agent, reference is made to the subject's freedom; with the term actor, reference is made to the social role covered by the subject. Actors are subjects that act in the roles of functional systems. Agents are subjects who act in systems of interdependence, both interactive and structural. The harm that market agents/actors cause to "distant" others can come from functional systems as well as from systems of interdependence, but their moral responsibility is different.

DOI: 10.4324/9781003146698-6 
The "others" can be single individuals, vast social groups, and even entire populations. Moral responsibility can be direct, indirect, or adiaphoric. ${ }^{2}$

It seems relatively easy to identify direct moral responsibility when, for example, a financial trader directly sells tainted financial products without informing clients of the elevated risk of loss, or when the owner of a sweatshop exploits underage or female workers, violating basic human rights. In these cases, Ego inflicts direct harm on Alter, who is a proximal (near) person, which does not exclude the possibility of harm done to third parties as well.

But what can we say when the economic action is done with the intention of harming no one, yet causes negative effects? Take the example of the decision to move investments to a new place to reap more profits when this decision involuntarily causes unemployment and poverty in other locations.

Traditional moral theory usually applies the principle of double effect (or the principle of the indirect volunteer). As commonly formulated, this principle establishes that it is possible to legitimately allow or tolerate a bad effect that comes about through an act of choice if four conditions are met:

1 The action in itself, apart from the harm caused, is good or at least indifferent (e.g. moving an investment from one place to another).

2 The good effect of the action is what the agent directly intends, only allowing the bad effect to happen (e.g. the unintended effect of unemployment).

3 The good effect must not be obtained by means of the bad effect (in the example under consideration, this is possible).

4 The path must be the only one possible or, in any case, there must be a proportionately grave reason to let the negative effect happen (in the example, this is problematic). ${ }^{3}$

If these criteria are met, the individually honest investor can feel at ease. But is this really how things are?

The moral criterion of the "indirect volunteer" presupposes a "linear" society, where the intentionality and causality of social action can be established with certainty. However, in a society in which interactions and relations are increasing out of all proportion, with marked circularities and long causal chains, this principle goes haywire.

2 Adiaphoric responsibility is "technical" responsibility, which cannot be judged in the strictly moral terms of good/evil. Here, I am making reference to the "adiaphoric society" spoken of by Bauman (1993) and to the "adiaphoric company" addressed by Jensen (2010).

3 J. L.A. Garçia, Double Effect, in Encyclopedia of Bioethics, New York, NY: MacMillan, 1995, p. 637-639. 
A society in the process of globalisation increases the cases in which the morality of action is problematic because action has a multiplicity of effects that, for the most part, are not grasped by the individual subject of the action. The reason for this resides in the fact that the reticularity of society is increasing significantly, that is the production of effects that are the outcome of complex social networks is increasing.

There was a time when people wondered whether the moral responsibility for poverty should be imputed to the fact that the poor are lazy, incapable, or maladapted or, instead, to certain economic and social structures. The social sciences have demonstrated for some time now that agents/actors' (direct, indirect, or adiaphoric) moral responsibility can never be separated from the societal structures within which they operate.

The answers generally offered by economists consist in claiming that we are dealing with the unintentional consequences of free market "laws." Harm is conceptualised as "side effect" or "negative externality." Harm caused to distant others is held to be the inevitable consequence of freedom as the guiding value of the social institution of the market and, thus, a contingency that is intrinsic to it. Redress can be thought of only ex post once rock-bottom has been reached. ${ }^{4}$ In democratic regimes, one expects that ex post consequences will be tempered and countered by political systems according to the societal arrangement that I call lib/lab.

The social sciences, however, point out that the market is a structure (or system) endowed with an ontological reality (Elder-Vass 2009) which, in a necessary and intrinsic manner, unintentionally harms distant others in a variety of ways, whether within single nations or in the relationship between developed and developing countries. These effects are called "unintentional structural effects," precisely to indicate that they depend on mechanisms that elude the power of individuals. When they deviate from the actors' intentions, they are called perverse effects (Boudon 1989).

Yet we wonder: is it really true that these mechanisms are inevitable and the effects unexpected? If it is true that these structures do not depend on the power of individuals, can we assert, for this reason, that harm to distant others does not have to do with the moral responsibilities of the agents/ actors?

Hard (holistic) conceptions and weak (individualistic) conceptions of the social structure both end up nullifying the moral responsibility of agents/ actors. System theorists shift responsibility on to societal structures (defined as adiaphoric). The theorists of individualism undermine the concepts of structure and agents' moral responsibility relative to social facts.

I do not share either of these two explanatory modalities. My thesis is another one.

4 This is the logic clarified by Teubner (2011). 
The social mechanisms or devices that cause certain effects (such as harm to strangers) are not a structure (either strong or weak) that determines agents/actors' behaviour in a compulsive or nominalistic way. Social mechanisms are relational, in the sense that they are made of social relations that are enacted by individual or collective subjects. Societal structures are institutionalised social relations, which are the product of networks of relations. For this reason, there exists a moral responsibility on the part of individual and collective subjects for outcomes, even for the unwanted outcomes. However, in order to see what is at stake, it is necessary to understand what is meant by the fact that social structures are built by individuals, but are not made of individuals in as much as they are made of relations, and it is here - in the relational dynamic - that the problem of moral responsibility is to be found.

For example, poverty, unemployment, and inequality brought about involuntarily are the product of networks of social relations: indeed, they are themselves social relations in which all actors, whether close at hand or distant, share, in different forms and degrees, some moral responsibility.

Yet we must be careful. If we adopt a relational perspective, there is the risk of falling into positions of circular relativism. The risk is that of locating moral responsibility in a sort of hermeneutic circle in which subjects' and structures' responsibilities alternatively chase after each other and merge (central conflation).

George Soros offers an example of this when he says that economic structures are produced by the expectations ("reflexive rationality") of financial operatives (on the stock exchange, in particular) and, at the same time, consist of such expectations, which discount the future. In short, it is assumed that the structures of the financial markets are made by the reflexive truths of financial agents, who reproduce them in terms of expectations, so that market outcomes can be completely irrational without it being possible to clearly attribute the causes to structures or agents.

This is the danger of "relationism," that is of adopting a view that locates responsibility in the pure circularity of relations among market subjects, which create the economic structures by which they are defined as market agents/actors. In order to avoid relationism, it is necessary to adopt an authentically relational perspective, which consists in identifying the specific responsibility of each term of the relations and, at the same time, the causality imputable to the subjects' relations as emergent effects.

\section{Questioning the prevailing explanations of causality}

In the field of sociology, holistic explanations seem to prevail. The most widely held version explains structural effects as expressions of functional requirements. It is claimed that also unintentional effects fulfil certain functions that are necessary to the social system. Therefore, there is no responsibility of single social actors, whether individual or collective. If the law of 
competition demands that the prices of merchandise be kept low, the individual entrepreneur has no choice but to pay lower salaries and resort to lay-offs, without worrying about the consequences for workers' families. It is noteworthy that functional systems give primacy to the adaptive function (A of AGIL).

The possible impersonal social harm brought to bear on distant others is seen as inevitable, and even morally indifferent, because it is essential to the good functioning of social systems. A certain number of unemployed and poor workers are considered to be an inevitable systemic factor for which market agents/actors do not bear responsibility because the economic system only works if it is possible to resort to the mechanism that uses a certain share of the labour force entering and leaving the labor market, as required by contingencies. Is this holistic point of view acceptable on the scientific level? In my opinion, it is not acceptable once the so-called functional imperatives (or the alleged laws) of the system are demonstrated to be neither necessary nor inevitable.

In the field of economic theory, the opposite point of view prevails, inspired by some version of methodological individualism. A great many mainstream economists hold that structural effects are "perverse" effects (unexpected and unintentional) due (i) to deviations from market mechanisms' optimal functional mode (which is equal to perfect competition), and (ii) to potential and concomitant defects in the regulation that should be guaranteed by political systems (with legislation on the labour market and the redistribution effected by the welfare state). According to prevailing economic policy, the perfect market should not produce negative structural effects - such as poverty - but only positive structural effects - such as legitimate or deserved inequality. Harm to distant others is considered an unwanted effect. The recipe for avoiding social harm thus consists in (i) correcting those market mechanisms that do not correspond to perfect competition, (ii) correcting welfare state mechanisms that do not guarantee equality of opportunity for individuals in the market and that, on the contrary, generate poverty traps and other harm.

The outcome of this logic is always to resort once again to compromise between state and market. According to this reading of the processes, individuals do not bear either direct or indirect responsibility for the impersonal harm brought to bear on distant others. The responsibility for social evils falls to the political collectivity, which should provide redress through impersonal measures (l'État-Providence as a safeguarding principle that lightens the burden of individuals' personal responsibility, or completely relieves them of it).

This solution is not satisfactory for many reasons. First, it risks making single agents unaccountable. Single agents do not have to change their logic of preferences and can shift their personal responsibility on to the collectivity. Second, it is not satisfactory because, as the history of the welfare state demonstrates, the solution that proposes to redress social evils by 
socialising harm through a continuous broadening of social citizenship of a lib/lab type multiplies the state's pervasive powers without civilising the market, given that it reinforces these same market mechanisms.

\section{Do alternatives exist?}

In my opinion, we need to begin by acknowledging that markets have become complex systems that no longer answer to linear logics. Systems develop networks that are black boxes in which each social relation is a structure that is refractory to its environment, so that the quality of that relation is regulated inside the system without it being possible to see the consequences on the outside.

Traditional ethics finds itself in a position of difficulty precisely because it is still anchored to linear thinking, that is to finalistic principles and to principles of linear causality, while market systems have expunged finalism and operate via functions and without a principle of linear causality.

It is easy to see the conflict between the functionalist perspective and the humanistic perspective. The former holds that all that which is possible for agents/actors is ethically licit, without it being possible to guide the system ethically. For the latter, instead, social structures, being the product of human persons, can be changed by giving them an ethical finality.

What hope does the humanistic perspective have? Or put another way: is it possible to alter market rules by introducing ethics as an independent variable that functions as a constraint on economic activity, considered not only in the single act, but in the causal chain at a distance?

Apparently, the confrontation between a functionalist and humanistic perspective is impossible to win for the latter, because ethics cannot stand alone against the instrumental aims of the market, and also because market's perverse effects can happen even if agents/actors are personally honest and the market's rules respect ethical constraints. If we conceive of the markets as functional systems, neither the ethics of business (see the regulations introduced in national and international financial systems after the crisis of September 2008) nor an organic ethics (such as that of the traditional Catholic Church teaching) can be successful. The answer can become affirmative if we realise that markets can be observed no longer as functional systems, but as systems of interdependence, characterised by a growing interactionality and relationality. This is what the relational paradigm proposes.

\section{In which direction should research be taken?}

Let us start over again with the question: who does bear moral responsibility for causing impersonal harm to others?

Certainly, the interactions among market agents/actors matter. But exactly how do we define these interactions and their outcomes? 
We must first reflect on the fact that the points of view of methodological holism and methodological individualism lack relationality.

As regards the holistic position, it should be pointed out that the functionalist theory of social systems encounters insurmountable limits and no longer functions beyond its own restricted operative domain because human persons are not an irrelevant environment for systems' autopoietic mechanisms. People assess and judge these mechanisms and can react reflexively on their modus operandi (voice and exit). Agents/actors feel responsibility for the devices that run systems despite (or perhaps because of) the fact that system mechanisms operate "impersonally," that is, by giving priority to adaptive requirements (A of AGIL) as a function of preset goals ( $\mathrm{G}$ of AGIL), with no concern for problems of legitimation and of social integration (the dimensions of latency and relationality, the L-I axis of AGIL).

As regards the individualistic position, I point out that the Coleman boat that determines market results (Coleman 1990) is not made of individuals who can act according to their wishes and even less according to the thinking of rational choice theory. The network that connects them is not a reality that they can alter to their liking on the basis of individual preferences and tastes. The network cannot be configured on the basis of a purely internal reflexivity of participants because it has to do with the dynamic of social relations as such.

We need a second-order observation that reasons about relations in terms of reciprocity and, for this, is able to observe agents' responsibility in building certain networks rather than others. In short, the moral responsibility of agents for the impersonal harm brought to bear on strangers becomes visible when the former are not considered as atoms, but as relational subjects.

In such a case, it becomes plausible to understand that there exists a moral responsibility of agents, if and to the degree that (i) agents consider the effects of their social relations, in as much as they are distinct from technical relations, and (ii) they adopt a vision of the market that makes manifest the ethical value (criterion) that is used latently as the argument for the function-goal of economic activity.

\section{The market as a black box}

Let us observe the impersonal damage to distant others as an outcome brought out by the process that brings from a certain distribution of $\mathrm{N}$ actors to another distribution, passing through the modification of the network of their market relations (i.e. the black box).

At the beginning of the process (time T1), we observe $\mathrm{N}$ agents/actors, embedded in a social context that influences their opportunities and action preferences, who start interacting. The context is a structure produced by previous morphostatic/morphogenetic (MS/MG) cycles. This structure defines the positions and resources of the $\mathrm{N}$ agents/actors in the 
initial distribution of their conditions (X). In the interval of time T2-T3, the $\mathrm{N}$ agents/actors make their individual and reciprocal choices, so that the choices combine with one another generating a network of relations between the $\mathrm{N}$ agents/actors. This network is a black box that operates the mediation between the initial distribution of the $\mathrm{N}$ agents/actors (X) and the resulting distribution (Y) which emerges at time T4. It is a matter of knowing this network (black box) and understanding its structural, cultural, and agential dynamic. In distribution Y, we see what the market has produced in terms of better or worse conditions among the $\mathrm{N}$ actors involved. We can find the relational goods and the relational evils that the black box (the market) has produced.

To see the effects on other distant ones, the market needs a third party to act as a sort of supervisor. This entity has the task of assessing the responsibilities in generating harms to distant others and intervene into the market process. It is necessary because (i) the morphogenetic process cannot be "finalised" ex ante (harms are emergent effects), (ii) the black box is not trivial. At the same time, it cannot be constituted by a single entity external to the overall process, be it an international organisation, an international network of NGOs, advocacy, or third sector organisations, consumer or worker organisations. It should be a relational network of the main stakeholders. Here is the point where the for-profit sector and the non-profit sector must cooperate through agreements that empower a third party - to which they rely because it represents them both - to evaluate the results of the process and to propose changes in the various aspects of the process itself. What this network of stakeholders can do is change the initial conditions, the black box, and/or the resulting distribution.

This requires a deep change in the dominant economic ethics, which considers the $\mathrm{N}$ market players as autonomous and rational - and generally hypo-socialised - subjects who are ethical in as much as they respect the regulations in economic transactions (lex mercatoria). Such an assumption forgets that subjects are not truly autonomous because (i) their interests, preferences, and choices are already embedded in particular contexts; (ii) their rationality is quite limited by the system's complexity, and (iii) there are many non-rational factors at play.

In order to find a solution to the problem, it is necessary to avoid seeing the causes of harms to distant others only in individuals or only in (social, cultural, and economic) structures. Theories of exchange on the free market and theories of hierarchical structuring are losing their explicative capacity. Harms to distant others are increasingly caused by impersonal, remote networks in which agents/actors' moral responsibility takes on an indirect or adiaphoric connotation. The question is, if, on one side, free inter-individual exchanges and, on the other side, organisational hierarchies give way to "social networks of stakeholders," what will be the fate of moral responsibility? 


\section{The moral criteria for imputing responsibility: the role of reflexivity and of the relational subject}

Let us ask ourselves how we can understand the causal connections between an average market agent's action and its effects.

In the case of lib/lab theories, things are quite clear. Agents make their choices from among the opportunities offered by structures and with the degree of freedom allowed by the structures. This theory emphasises the pressure of structures on individual actions. According to this theory, agents act in a certain way even if they are aware of the harm that they can cause because the environmental pressure is so strong that they would otherwise be labelled as deviant (Blau 1960) and thus would be marginalised from their social group of belonging. The black box works in such a way that harmful outcomes are imputed to structures, not to agents.

The solutions of voice and exit emerge from those who discern that distribution $\mathrm{Y}$ of the opportunities that emerged at time T4 is not morally (and in certain cases, nor politically) acceptable. At this point, to whom is responsibility to be imputed?

The ethics of traditional cultures judges results in a deductive manner, ex post, with a retroaction that does not see the social causes of the process; it limits itself to judge the actions of the $\mathrm{N}$ market actors and asks to change their preferences and choices. But how can responsibility be imputed to individuals if the causes of the harm to distant others are not clear? Asking to compensate those who have been harmed with a public intervention or with an appeal for private charity does not alter the black box.

If the process must avoid generating relational evils and producing relational goods, the black box must be modified, making the process more reflexive on how it operates. Making the process reflexive means to "turn back" and identify the responsibility of who and/or what (social mechanisms) have operated in the black box so to produce the social evils. This means adopting a relational ethics.

In order to understand how structures can be changed, it is necessary to understand how they are causally generated.

Structures are made of relations, and relations are made of reciprocal actions (with their intentions, norms, means, and values). What we call "market rules" are relational structures. Precisely, due to their relational characters, they generate an "other" reality with respect to the individuals and structures that we observe in a given space-time. This "third" is constituted by emergent effects, which traditional ethics does not see because it judges individuals and structures. It is here that ethics must make itself reflexive or, rather, meta-reflexive, in the sense that it must judge the morality of actions in light of how they configure relations, leaving aside agents' intentions and existing structures.

This means that the ethical criteria that assess market functioning and its products must make reference to social relations rather than to impersonal 
system mechanisms or individual preferences, possibly encouraged by incentives to carry out cooperative games. In any case, causal imputations can no longer be of a deductive and conditional type, of the type whereby, "if event X happens, judgment Y then applies."

It is necessary that the ethical criteria for imputing moral responsibility concern agents not only as regards their individual subjectivity (internal conversation or personal reflexivity), but also as regards their social relations. Agents must reflect on the relations that condition them and on the relations that they generate. Relational good can transform itself into a relational evil if a suitable social (i.e. relational) reflexivity and a certain system reflexivity are not activated. Ethics must make itself relational. What does this mean?

It means seeing good and evil in social relations and not only in the individuals as such or in the conditioning structure. To understand the significance of this statement, one should think about the following examples.

Traditional ethics considers poverty as a condition lacking resources that should be given by the collectivity to those who are most unfortunate. Relational ethics, instead, sees poverty as the product of relations that, even if enacted with good intentions, have objectively deprived some people of opportunities to which they had a right for reasons of reciprocity. Likewise, unemployment is seen as the product of policy that considers work as a commodity rather than as a social relation (Donati 2001). It is certainly true that, since last few years, the UNO has learned to conceive of poverty as a lack of capabilities, which leads to exclusion from the circuits of productivity. Amartya Sen, whose thinking has contributed to this new view, has proposed that the conception of poverty as a pure and simple lack of income be superseded. Nevertheless, concepts such as a "lack of capabilities" or a "lack of flexibility in the job market" usually make reference to individuals as such, that is they reflect an individualistic point of view, or they reflect a collective point of view, making reference to impersonal structures, rather than being expressed as forms of social relations. This is due to the fact that the common good is defined as a "total good" (a product of aggregation) rather than as a "relational good" (emergent multiplier effect).

In general, what I want to claim is that involuntary, but illegitimate, inequalities must be seen as the product of a relationality among actors who lack reflexivity in the mechanisms that distribute life opportunities, even if those who participate in the exchanges are individually honest.

At this point, a clarification of the concept of ethical good (and, conversely, the ethical evil) that I am proposing is called for. The idea that ethical good consists in the flowering of the human person in happiness is widely shared, together with the assumption that happiness is due to the personal virtues of people. The opposite idea applies to ethical evil, seen as the result of personal vices. In this view, the normative components of social relations that lead to a happy or sad life are a consequence of persons' virtues or vices.

What I add to this conceptualisation is a more complex view of the relationality inherent in social morality. The social dimension of the morality of 
action does not only consist in the fact that the person must relate to others in a virtuous way with his/her own intentionality (necessary condition). It also includes the person's responsibility for the consequences of the relation as such on other relevant persons (sufficient condition). Social morality is good if this second condition is met, that is if the effects of one's own action on others are considered. In this consists the social nature of virtue, differently from personal virtue.

This is relational ethics, which joins together intentionality towards a good and responsibility for the relational value of the good as it is expressed in its consequences. The ethics of intentionality and the ethics of responsibility cannot be separated, precisely due to the relational character of the good or evil. We go beyond modernity to the extent that we attribute a responsibility to subjects not only for what they think or do individually, but in as much as they generate social relations that have a sui generis reality leading to certain consequences. This means conferring an ontological statute on the concept of relation when it is applied to the social realm. The morality of an action does not reside only in the conscience of the subject acting with an eye to a good in itself, but also to the fact that the subject does or does not respond to the relational nature of the sought after good.

\section{The relational subject}

The "discovery" of the reality of the social relation leads to a new ethical reflection when we consider that the relational good/evil is created in the interactive network (black box) in the interval between times T2-T3. Let us look at what happens inside the black box with the help of the morphogenetic framework (Figure 6.1).

Time1 - Starting social structure conditioning individuals’ morality

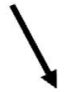

(individual ethics: agents are positioned within the initial structure and act accordingly to their individual choices)

Time2 - Interactions between agents (agents are required to reflect upon the positive / negative externalities of the network they create)-Time 3

Temporal phases (relational ethics, depending on the constitution
of a relational subject $)$

Time4 - Emergent structure in which moral responsibility is up to the agents as relational subjects, i.e. as responsible for the effects of their network on distant others

(a new cycle begins)

Figure 6.1 The interplay between individual and relational ethics

Source: Author. 
Figure 6.1 is entitled "the interplay between individual and relational ethics" to underscore that relationality changes moral responsibility over time. Morality is not given, once and for all, in the initial decision. It does not only concern single acts in time, but has to do with these acts in as much as they produce relational effects over the course of time.

At the initial time T1, individuals find themselves in a structure that conditions them, and they make their choices. Traditional ethics considers the morality of the individual acting according to his/her inner reflexivity in response to the social context. At time T2, individuals' choices interact with the choices of other agents. A network is constituted among "proximal" individuals (proximity network). In this phase T2-T3, moral responsibility concerns each agent due to the contribution that he/she makes to the network. In order to see the connections with external others towards which there is the responsibility of positive/negative impacts, the constitution of a relational subject is required. If a relational subject can be actually generated through a morality that exercises its reflexivity on relationships (relational ethics), then a new social structure is generated consisting of a social network that seeks to avoid harm to distant others or at least to remedy it. Obviously, the problem is not solved once and for all. Here begins a new cycle in which agents will have to make their individual choices in response to the new structure.

Decisions in the proximity network affect the outside, even in an unintentional way, because there exist structural (unintentional) connections. Only if agents pass from their individual morality to take the point of view of the relational effects of their actions, that is if they become relational subjects, is it possible to avoid damage to other distant people. Otherwise, relational evils are inevitable. As we will see in Chapter 8, the relational subject is one who acts taking into account the fact that relations can produce relational goods or evils.

For example, when white workers in the United States prevented Blacks from joining labour unions, they did not do this to prevent them from working - or even due to racism - but because they believed that Blacks would not be loyal to the union and, therefore, they thought that they were defending their legitimate interests. They had "good reasons" for their actions. Nevertheless, the emergent structural effect was to produce unemployment and poverty among Blacks, as Merton (1968: chapter 2) has shown.

From the standpoint of relational ethics, moral responsibility implies the duty to know the relational connections between the networks in which the agent is integrated (IN) and the relevant environment. Yet studies to this effect are quite rare because the issue of knowledge on how relationships operate is fairly new in social research. However, it is possible to make several assertions that are validated by network relational analysis.

For example, we can say that moral responsibility is proportional to the degree of the Ego's centrality in the network. Nevertheless, whoever occupies 
the position of broker has a supplementary responsibility, because the broker's responsibility is not only towards the network's individual nodes (each Alter), but also towards the network overall in as much as what the network generates depends on the way in which a broker manages the structural holes of the network.

It should be noted that the relational interpretation of the principle of responsibility is quite different from the one formulated by Hans Jonas, not only because Jonas has addressed this principle mainly as it relates to phenomena of the natural environment (ecological and bioethical issues), but above all because he has applied it to individual action in the spirit of Kant ("Act in such a way that the effects of your action are compatible with the continuation of an authentically human life"). What distinguishes relational ethics is the fact that actors must act reflexively on the results of their actions combined (related) with others' actions, and not only on the consequences of their own individual actions. In order for this to happen, it is necessary that a moral relational subject, to which moral criteria are applied, be constituted.

Undoubtedly, some connections are known and certain, while others are not known, or are uncertain. But ignorance is not an excuse for refusing to take responsibility. In many cases, the "remote" causality of the market's economic phenomena is identifiable only if we presuppose the existence of a relational subject with respect to which agents are responsible. This is because it is with respect to this relational subject that an observer can assess the morality of their actions, whether ex ante or ex post, in producing those causal chains from which harm brought to bear on distant others is derived.

For example, a company that hires women with no concern for their relations with their babies is potentially responsible for the harm suffered by the latter due to the lack of maternal care. A company cannot ignore the network of relationships that its workers have in the company environment (of course this principle applies equally to men and women). Most probably many companies do not have bad intentions, but nevertheless they produce relational harms in the human environment of the territory in which they operate. Relational reflexivity on the process requires one to see the company, the employees, and their families as relational subjects. When one says that employers have a social responsibility towards their employees, this means that they should consider potential harms to employees' families due to the type of work done. The solution is to make relational contracts that provide for family-work reconciliation measures. The contract is said to be relational precisely because it considers the relational effects of work on family life that lie outside the so-called contractual synallagm in which a simple equivalence is assumed between services rendered and remuneration of the individual employee's work. The relational contract is made not between two individuals (traditional ethics), but between two relational subjects (relational ethics) 
The relational perspective is not wishful thinking but has empirical corroboration in a number of new ways of running the market that follow relational ethics. For example, many companies prevent harm to distant others by building networks in which for-profit and non-profit subjects cooperate, and for this are called socially responsible.

We are witnessing the birth of markets in which agents' behaviours and rules are being redefined from the point of view of the relational subject constituted by a broad spectrum of stakeholders (business ownership and management + workers + consumers). New forms of relational economy are being born, integrating for-profit and non-profit sectors and holding as a value the social responsibility of business (CSR, shared responsibilities) and the production of commons. More recent than the concept of CSR is that of "shared value," used by Michael Porter as well as Mark Esposito, Rosabeth Moss Kanter, and others, which has the advantage of using economic language in the sense that it underscores that the goal of business is also value and not only profit. Social and positive ecological effects are included in value. Running through all of these innovations is the observation that the company that pursues only monetary profit is destined to fail.

These configurations are characterised by the fact that they introduce what I call "relational reflexivity" (Donati 2011b) into the black box and, in this way, civilise the market, while the typically capitalistic configuration which is based on an acquisitive autonomous reflexivity - commodifies it.

\section{Some examples}

If market participants do not understand harm brought to bear on distant others as a product of their actions, it is because they do not see, or refuse to see, the relations that connect them. The prevailing political economy of today immunises them against these relations. How can one respond to someone who refuses to consider relations? How can we address someone who wants to have to do with us only for the things exchanged and not for the relations entailed by the exchange?

Let us look at some examples.

\section{One: Social dumping}

Social dumping is a practice considered in the economic world to be unfair. But in point of fact, in the United States, it is a normal praxis backed up by the laws on competition, and in the Europe Union, it is politics backed up by European Commission policy directives meant to foster market competition among member states. The lib/lab system imposes a market structure based on social dumping. And so one wonders: what responsibility does the individual business person, who practises social dumping, have in generating harm in those countries or areas where it causes unemployment and endangers workers' social rights? 
From the perspective of relational sociology, conformity to starting structures does not absorb all of the entrepreneur's moral responsibility because the latter has degrees of freedom in seeking alternative solutions if and when he/she wants to avoid unintentionally causing harm. Entrepreneurs can adopt cooperative strategies aimed at building social networks linking the countries or areas involved so to avoid harms to distant others. It is in this way that a civil economy arises that respects the ethical criterion of reciprocity as the moral rule of relations.

\section{Two: Acquisition of merchandise produced in violation of human rights}

What moral responsibility does the consumer have when he/she buys merchandise produced with the exploitation of child labour or with the low paid work of women operating in "sweatshops" in developing countries?

The answer lies in the reflexive awareness of consumers. If they have no knowledge of the fact, we cannot impute moral responsibility to them. But if the network of corporate agents makes this reality public, and a consumer comes to know about it, he/she has the freedom to choose what to do. And so the knowledge of exploitation is an element in the imputation of responsibility because the consumer has the possibility of not buying sweatshop products.

\section{Three: The rejection of risks that entails fostering the bankruptcy of countries in financial crisis}

Let us take the case of a financial agent who withdraws his/her financial investments from a country in which there are risks of fiscal and financial instability. By acting in this way, the agent contributes to causing unemployment and economic recession in that country. Such an agent is generally perfectly aware of what he/she causes, but imputes harm to the laws of the market. Moral responsibility lies above all in the refusal to alter the network of interactions, which can produce harm to distant others. The responsibility is thus proportional to the degrees of freedom available for altering the network of financial actors in such a way as to avoid both one's own losses as well as harm to others. Surely, we cannot criticise a person who tries to save his/her modest savings. On the other hand, from a moral standpoint, one must reserve a negative judgment for rating agencies which, with their evaluations, contribute to increasing the difficulties experienced by those countries that already have weak economies and, thus, instead of helping these countries to get back on their feet, they favour dynamics that lead these economies towards situations of even deeper crisis (they are examples of self-fulfilling prophecies).

\section{Four: The involuntary creation of poverty}

From the standpoint of sociological analysis, the condition of poverty is not a condition that is caused solely by certain individual characteristics, 
but is above all the product of a relational system. Addressing poverty as an emergent effect of networks of relations completely changes the perspective as compared to an essentialist point of view that considers poverty as a condition caused by material (economic or physical) factors which have determined a certain outcome, that is the lack of means. The struggle against poverty in the market - without intervention by the political system - consists in creating a new interactive network of a cooperative type.

"Social causality" is a relational causality. Life conditions and opportunities are the outcome of a relational context that not only conditions individuals' choices, but also has its own logic of interdependence that generates social problems. The category of interdependence is a moral category in which it can generate relational goods or evils. The moral responsibility for the creation of poverty does not have to do with the individual behaviour of single persons, but with the consequences of the relations that they activate. They are responsible for the outcomes because they are responsible for the relations that generate them, even if these are emergent effects that come about without their direct intentions.

The poverty of the homeless man on the street or of the illegal immigrant is the result of a long chain of actions that could have been individually legal and honest, but which did not take into account either aggregate or emergent effects. When I see an old lady collect her pension at the post office, she does not know that this money comes from me and from those who are currently active in the work force (Ignatieff 1984). But I know it, and I am responsible for it. If I do not pay my taxes and other contributions to the collectivity, if I do not share in the We-relation and the relational subject that supports it, I am responsible for the failure of the collectivity to pay this lady's pension.

\section{Summary: can we civilise the market?}

The idea that the market is a system of exchanges where the golden rule is pure competition, that is, with no premises beyond the mere capacities of individuals, is a myth. It is a pure illusion due to the simple fact that it erases the relations between market participants. Competition is a social virtue if it means cum-petere, that is, to strive together towards a good that entails the benefit of all those involved.

The modern political economy that originated in the 18th century, which in general terms theorises the market as "formally free" and, as much as possible, "deregulated," is based on the removal of social relations because of its individualistic theological matrix. This model has historically triumphed over the idea of a civil economy (Genovesi 2005 [1765-1767]; Bruni and Zamagni 2004) based on social relations. Although Adam Smith spoke of sympathy among market actors, it is quite true that all political economy from Ricardo onward is pervaded by ethics of "immunisation" of market agents against social relations. 


\section{Insights into the morphogenetic changes}

Since the economy is effectively relational, its ethics must also be relational. Redistributive economies (based on a central authority) and the so-called social market economies (which are a form of state regulation of the market) do not properly have a relational character and, for this reason, fail in combating the harm brought to bear by the market on distant others. At most, they can bring some relief.

In any case, in order to avoid harm caused by the market to distant others, recourse to the state alone is inadequate. Rather, mechanisms of civil society are needed, if and in as much as they are relational. An economy is relational not because it entrusts itself to the regulations of some political system, but because it invests in solutions based on the social governance of civil society's networks.

With this, we have reached the crux of the problem: the morality of actions in the market is essentially a problem of civilising the market, considered as a network of interdependences and interactions.

Ethics can operate as an independent variable or a function-objective of economic activity if it is conceived according to reflexive criteria, and no longer according to unidirectional criteria (i.e. the conditional criteria such that, if $\mathrm{X}$ happens, then the norm $\mathrm{Y}$ is applied).

In a society that is exploding agents' subjectivity and creating always new relations via the channels of globalisation, it is no longer enough to say that the morality of action depends on the conscious intention to pursue something good in itself. Unfortunately, social evils do not derive only from behaviours that are dishonest, corrupt, and driven by greed for money or power - which are clearly visible, they derive from social networks that do not operate with adequate relational reflexivity. 


\section{Part III}

Why and how can the new society be "good"? 


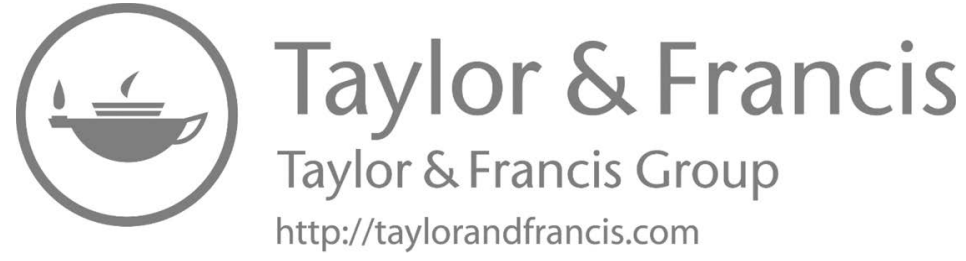




\section{What does the good life consist of?}

\section{What is a "good life"?}

I am interested in shedding light on practices that are inspired by a realist utopia that uses opportunities in order to realise a modus vivendi that allows people to enjoy relational goods in different social spheres. In short, I would like to highlight those social forms of the good life that are capable of including new life opportunities within the social relations that orient our conducts of life towards an "agonistic sociability." This oxymoron alludes to the fact that, instead of encouraging citizens to bracket their moral and cultural disagreements, we have to cultivate oppositional yet respectful, that is relational, civic, and political practices. In the field of social services, this means developing relational social work. I argue that a flourishing civil society, on which a civil democracy is grounded, can be fostered by those social networks that are able to generate competing relational goods.

My argument is that in a society conceived as a field of opportunities, the discriminating factor of "living well" becomes the relational or non-relational nature of the good that is sought and realised by the acting subjects. It is a matter of clarifying the modalities with which the good is generated and which effects follow from it. The proof of this argument consists in giving evidences that there exists a specific logic of opportunities that is capable of realising a "society of the human," that is social forms in which, whatever the means used to realise social relations, the latter can be generated only by subjects who are actively oriented to each other according to a suprafunctional sense. This is a society in which, from the standpoint of relational realism, the good life coincides with the creation and enjoyment of relational goods.

\section{Classical and modern conceptions of human happiness}

There are two alternative views on what human happiness might be, which have prevailed over the centuries: a hedonic idea of happiness and a eudemonic one. 
For the hedonic conception, happiness is the result of avoiding pain and seeking pleasure, the key concept of all utilitarian schools, in both its individual and aggregated forms. Social relations are considered as "entities" that can bring pleasure or pain as other "objects" do.

On the other hand, we find the eudemonic view, which, apart from being more theoretical and holistic, takes a different view of human relationality. It considers happiness as a more complex concept, not strictly limited to attaining pleasure. Happiness is something like flourishing human living, a kind of living that is active, inclusive of all that has intrinsic value. It is the ultimate goal of human life and an indirect result of the practice of virtue.

I will focus on the latter conception, starting from Aristotle, who claims that pleasure is an enérgeia of the human body and mind, whereas happiness is the enérgeia of a human "being a human" (Aristotle, Nicomachean Ethics, Book X, Chapters 1-5). Aristotle understood human happiness on two levels.

On the individual level, as the satisfaction of the human being's natural needs (physical, psychological, and sociocultural), with the purpose of enhancing the more elevated human qualities, however one defines them (rationality, contemplation, otium, spiritual virtues).

On the social level, life consists in enjoying interpersonal friendship and in conducting a correlated active and peaceful life in the public sphere of the polis, with the intention of pursuing the common good. Aristotle does not examine in detail the relational nature of good life, limiting himself to making individual happiness dependent on the happiness of the political community, the latter having primacy over the former. Throughout history many other conceptions have been formulated on this basis, which certainly need not be summarised here. On the one hand, human needs have been discussed at great length, and, on the other, the relationship between individual happiness and collective happiness has been variously described.

What I wish to recall is the fact that in classical thought and until the beginning of modernity, good life is related to two basic conditions: (i) it refers to a naturalness of human needs and thus presupposes a human nature, however this is defined, and (ii) it implies that the political community is capable of pursuing the common good by resolving social conflicts and giving citizens the security necessary to enable their human potentialities to flourish. These potentialities are generally understood as virtues.

Virtue (in Latin, virtus, and in Greek, $\alpha \rho \varepsilon \tau \dot{-}$-aretè) is understood as a disposition of the spirit towards the good; in other words, it is a person's capacity to excel in something, to accomplish a certain act in an excellent way, to be virtuous as the perfect way of being.

In premodern thought, virtue has a stable disposition called habitus as its prerequisite. Habitus is a fundamental means for achieving the good life in that it regenerates a social order conceived as an ideal that is stable and immutable in its principles. From this comes the idea that a happy society, and a good life for its citizens, is achieved by a strict correspondence 
between personal virtues and social order, and that it is reproducible over time. The idea that individuals' happiness (as the realisation of their virtues) projects itself onto the entire society prevails so that if individuals, as such, act for the good and are happy, the society will also be happy.

This vision lacks relationality. With modernity, this framework is progressively called into question. To the degree to which the individual is no longer incorporated (embedded) in a given community and becomes "casual" (formally free and available in the capitalist labour market), the distance between the individual and society grows increasingly greater. With the advance of the national state and the spread of capitalism, the two assumptions of premodern thought fall: the notion of human nature and that of the common good are radically changed, altered, overturned, and, with them, the meaning of good life as well.

With the progressive erosion of the metaphysical-religious roots of the past, the problem of how to sensibly conduct one's life becomes an increasingly fraught problem. In a society in which the values that guide life are no longer "founded" but simply chosen with subjective options, good life presents itself as an always problematic and somehow unreachable goal. The fact is that all of modern culture, from its beginnings to the present day, is marked by the drama of defining "what is human" and consequently what is, or can be, human happiness (Archer 2000).

With post-modernity, the process of change becomes ever faster and deeper. What is the conduct of life that leads to happiness and, even more to the point, what is the society that can foster it? These are questions that are increasingly debated along two main lines: on the one hand, there are those who hold that the good life consists in the emancipation and liberation of individuals' subjectivity from any system constraint (a new form of the hedonic ethos); on the other hand, there are those who believe that it consists in the possibility of building highly techno-functional impersonal social systems that can relieve individuals from their material needs, which is a technocratic reformulation of the eudemonic ethos. In reality, Western modernisation mixes both the aforementioned tendencies: individualism and system functionalism mix and intermingle with one another. They support and feed off each other.

Social structures, once the possibilities of basing moral values in an objective reality are lost, now function as if the problem of living in a good society can be reduced to a question of individual preferences and tastes that are allowed or not allowed by the system. The happiness of individuals is relegated to the private sphere, where it is subjectified and becomes narcissistic (Lasch 1979) while public happiness (the well-being of social systems) is entrusted to the chance of creating "reflexive systems" (Beck, Bonss, and Lau 2003). Everybody agrees that late modernity requires more and more reflexivity in order to pursue happiness. A system qua talis cannot be properly reflexive; it can only be reflective in a mechanical way like in a mirror. So, we have to distinguish between different modalities of being 
reflexive, whereas Beck, Bonss, and Lau (2003) fail to make these distinctions. As Beck, Bonss and Lau (2003: 3) put it, "reflexive does not mean that people today lead a more conscious life (...) reflexive does not signify an increase in mastery and consciousness, but a heightened awareness that mastery is impossible. Simple modernity becomes 'reflexive modernisation' to the extent that it disenchants and then dissolves its own taken-for-granted premises." This leaves the referent, the purpose, and the point of reflexivity highly ambiguous.

Here it seems clear that the problem of human happiness is being posed in radically new terms, for at least two major orders of reasons.

1 Social and cultural systems no longer presuppose the existence of a human nature. On the contrary, they tend to alter existing reality to enter into the realm of the "post-human," the "transhuman." They create the humanoid and the cyborg. All prior forms of humanism become obsolete. In short, human happiness no longer consists in the realisation of potentialities that are proper to human nature and only to it, but exists "elsewhere," an "elsewhere" that cannot be defined because it does not have either an identity or stable boundaries. It is said that society becomes liquid, and people must live on the edge of chaos.

2 The processes of social differentiation erode the concept of the common good and, with it, the idea that good life can be guaranteed by a political community. The common good is identified sometimes with public goods, sometimes with total goods, that is with impersonal entities or entities created by a simple aggregation of individual goods. The idea that public happiness can avail itself of private vices, indeed, that it is the product, even if an unintentional one, of private vices becomes a social norm. The logic of the production of the goods in which happiness consists is left to the neo-liberalism of the market regulated by the state (lib/lab arrangement).

As a consequence of all of the above, happiness becomes a mysterious object, a dream, a passion, a conduct of life without a symbolic and normative centre. It is no longer a project. It is abandoned to the intrinsic ambivalence of a Western morality that puts everything into doubt and is thought of as being purely "liquid," while, in fact, it is not, due to the existence of tough structures of social inequality, in particular in accessing social service and in organising alternatives to the lib/lab arrangements.

In reality, this society does not see that the liquid life and the risks that hinder the possibility of achieving the good life depend on precise social and cultural structures. We can ask, where can such a society, which appears to limit itself to being aware of its own inability to solve the problems that it generates, find happiness? Where is the "good society?" Certainly it is not only in material well-being. Various scholars have evidenced the "paradox of happiness," which states that in the dynamic of advanced societies, 
beyond a certain threshold of material well-being, increases in income and material goods do not, in fact, lead to increased happiness but generate its opposite, that is unhappiness and a whole set of connected individual and social pathologies.

Many economists and psychologists of the so-called economics of happiness are still far from giving a convincing answer. In my opinion, the reason for this shortfall lies in the fact of not having really understood the role that social relations play in fostering human flourishing. The human being is a sui generis potentiality that can be actualised only through the relationality with other human beings. The central point becomes that of understanding how the logic of opportunities, which is supported by the morphogenetic society (Archer ed. 2013), puts social relations into a state of fluctuation and what consequences this has on the good life.

\section{The opportunities offered by the morals prevailing in modernity}

In the modern conceptions of the good life, goods are of an either individual or collective nature. In a nutshell, we can say that there exist two moralities of the good life that drive social changes, and a third morality that is generally considered auxiliary, complementary, and, in any case, residual compared to the other two.

Said in short, the two driving moralities are those of the capitalist market and the state (or political system). The morality of the economic market extols the ideal virtues of honest and efficient competition in producing a never-ending supply of new goods that are supposed to improve the wellbeing of individuals and society. As a matter of fact, these virtues are not actually practiced. What is really at work is the idea that a good society should allow agents to engage in their free and private activities by means of which they are expected to enrich themselves and the social body (liberal morality). For this morality, opportunities are created by the capitalist market. Of course, there are other kinds of markets, with different moralities, based on different norms of exchange. But it is well known that capitalism marginalises these different (civil) economies.

Collective morality, instead, extols the civic virtues of agents' participation in and responsibility towards the public good, which is identified in the total good of the redistributive state that guarantees the rights of citizenship and equality of material starting conditions (socialist morality). For this morality, opportunities are created by the state or the political-administrative systems existing at the different territorial levels.

The third morality, the most marginal one, is that of the social spheres in which the virtues are neither those of the market nor of political citizenship but make reference to relations of trust, cooperation, and reciprocity in lifeworlds. Opportunities are created in and by the primary and secondary social networks of civil society whose morality is based neither on profit exchange nor on redistributive norms, but on criteria of reciprocity 
(peer-to-peer production, coproduction, open coordination, partnership, etc.). The marginality of this third morality is attested to by the fact that its guiding-value (fraternité or solidarity) is not institutionalised in the cultural system (including the legal system) as, instead, the other two guiding-values are (liberté and égalité).

These three moralities refer to different logics of opportunity, which are opportunities in liberal, socialist, and "associational" terms. All three have their own specific conception of what we call life opportunity. The opportunities offered by the market, those offered by the state, and those offered by the networks of lifeworlds respond to different relational logics intrinsic to the three aforementioned moralities, respectively, of economic exchanges, political safeguards of citizenship, and associative relations. Each logic of opportunities reflects a different morality of social relations.

It then becomes a matter of analysing who offers the opportunities, how the opportunities are selected and utilised, and what their effects on the good life are.

\section{How globalisation redefines the logic of opportunity}

I wonder, which logic of good life opportunities dominates the globalised world? It seems that this logic is driven by an "aesthetic energy" that makes individuals choose favourable opportunities on the basis of a type of utility that is instrumental to goals that are the "interests of the moment," with no constraining finalities responding to a long-term project - and, thus, without norms that potentially make the choices stable so that individuals end up aggregating and disaggregating with a growing variability that no longer responds to any social order except that of expressing a diffuse spontaneity. Family structures and "family moralities" offer numerous examples of the variety of ways in which individuals aggregate and disaggregate.

These new situational logics of opportunity seem to correspond to an underlying impulse that we could call "collective addiction," favoured by the medium of an "anonymous communication matrix" (Teubner 2006a). It is a logic of the search for happiness through an unchecked availability of all possible innovations, which makes people addicted to continual change as if they were addicted to a drug. The process of societal morphogenesis takes the features of an "addictive society" (Teubner 2011).

Now the question becomes, to what extent is the good life pursued in a rational and reflexive manner, by whom and in which contexts? And where are new conceptions of the good life emerging in a non-normative way? What supports social integration? What produces social disintegration? In other words, is it possible that, passing through a phase of unbound and anormative morphogenesis (Archer 2016), new conceptions or effective social practices of the good life can be generated in which agents/actors find a stable consensus among themselves and build something in common? 
It seems to me that on this issue two main opposing arguments are advanced. One argument holds that the new prevalent moralities of the good life are the product of agents who are basically unconscious, driven by weak or fractured or impeded forms of reflexivity, that is modalities of action that characterise a passive attitude or a more laissez-faire outlook of "wait and see," without any ability to anticipate the outcomes of their actions and life course. The other argument claims that, nonetheless, the new conceptions and practices of the good life are the product of conscious and free agents who make rational decisions.

From the standpoint of the relational theory of society, which is neither relationist nor formalist, we see acting subjects faced with the need to confer a normativity on social relations that is adequate to successfully achieving the promise of a good life. This need can only be met with a minimum of adequate reflexivity leading to an agonistic understanding of normativity (Maxwell 2012). In other words, so that interactions between social agents can produce social cohesion that respects the rights of human persons, it is necessary for acting subjects to acquire the characteristics of contesting relational subjects.

People create social cohesion to the extent that they act as subjects who reflect on social relations as emergents and, without necessarily sharing the same tastes and opinions, are nonetheless able to build a We-relation. This entails understanding the meaning and practical implications of how a relational subject is constituted, whether this is a single person or a set of people who act as a collective entity or as social network.

\section{In search for a relational logic of happiness}

The morality of the lib/lab configuration of society is based on an injunction: "you must be free" to seek opportunities that fulfil you. This injunction is configured as a "double bind" that consists in one's being at the mercy of a paradoxical message: if you obey this injunction, you show that you are not free because you do it out of obligation; if you do not obey this injunction, then this means that you renounce being free. Apparently, there is no way to escape this paradox, which is notoriously at the origin of so many psychic and social pathologies and was elucidated as "the trap of postmodernity" by Michel Foucault (1966).

The relational paradigm argues that a way out exists. In order to see it, it is necessary to escape the paradoxes of modernity with a process of cultural breakthrough. This process consists in semantisising the injunction, turning its meaning upside down, that is by resorting to a counter-paradox: "you must be free" comes to mean that you must choose whom to depend on because freedom consists in having the possibility of choosing the relation to which to belong, the bond that, through your choice, is the foundation of your identity. 
To solve the paradoxical message of late modernity, one needs to look at the enigma of the social relation and be able to manage it. The enigma is inscribed in the social and cultural structures that impose on individuals the norm of having to realise themselves by making themselves independent of every social bond. From this system injunction derives a clear deception that consists in attributing to lone individuals the responsibility for everything that happens to them in life. Theirs is the fault, theirs the shame. From this comes the repressive sense of human and social relations in present-day society. This social norm is not saying, as some think, that the human person has the moral obligation to enhance his/her capacities: on the contrary, individuals are commanded to transcend themselves, to go beyond their capacities and potential to take on qualities and properties that the human does not have. This is the post-human, the hyper-human, the transhuman, and the cyborg.

In the private sphere, interpersonal bonds are replaced by technologies: for example, procreative relations are replaced by reproductive technologies; primary relations are replaced by virtual communication on the Internet; in the public sphere, collective bonds are replaced by system bureaucracies and mechanisms; in large organisations, the bonds between people are replaced by technological tools; in public debate, dialogue between faceto-face participants is replaced by mass media and new apps. In all of these cases, social bonds become increasingly virtual. What was considered the "natural" quality of the bond between human beings loses its meaning, is rendered artificial, and, as a result, the bond can be constructed and altered at pleasure.

The fact is that, when talking about good life, we have to reconsider what we mean by "human nature" and, correspondingly, what the demands that social and cultural structures impose on people's action are, especially with respect to the social bond. We have to understand the complexity of the morphogenesis of the human in order to grasp the novelty of the human wherever it is regenerating rather than destroying itself.

My thesis is that the regeneration of the human, wherever it is not being lost but is instead flourishing, emerges as the product of a qualified morphogenesis of the social bond. I would like to explain this statement by analysing the causes that make the transition from lib/lab morality to a relational morality necessary.

This transition starts when interacting actors take a distance from the system of opportunistic logics supported by the lib/lab arrangement. The sequence is the following: (i) first of all, variability increases within the lib/lab framework; (ii) the opening of new, purely contingent opportunities creates a space-time in which the search for new rules for the selection of alternatives takes place; these rules refer to relations that must be generated; (iii) if choices are enacted that, in a targeted way, guide the creation and use of opportunities according to new relational logics, stabilised social innovations emerge in which the goal of humanising these same social relations prevails. 
We can delineate the discontinuity between lib/lab ethics and relational ethics with respect to their creation and use of opportunities as follows.

In the lib/lab paradigm, (i) the ethics of good life is a private choice and becomes public only as an external constraint on action; (ii) the social quality of objectives and products is independent of inter-human relations because it makes reference to the achievement of the maximum of individual opportunities. The "advantageous" new forms of "variety" are necessarily appropriated by those who, even if they start from supposedly equal positions, have the capacities and means for appropriating them.

In the relational paradigm: (i) the ethics of good life pertains to the social relation in that it is a bond between humans, that is inter-human; (ii) the social quality is that which derives from the respect for and fostering of values and norms that give priority to caring for the relations between the acting subjects. In this case, the appropriation of "advantageous" new forms of "variety" by some to the detriment of others is hindered or very limited because here the moral norm of reciprocity, understood as symbolic exchange, is in force.

The aim of a symbolic exchange is to generate, maintain, or change a meaningful relationship with significant others. The easiest example of a symbolic exchange is doing someone a favour, giving a gift, or offering assistance. It can be a move to start a relationship of reciprocity, wherein reciprocity does not mean an exchange of utility (do ut des), or an act that regenerates an existing relationship. When the act is based on an already established relation, the favour, gift, or assistance maintains a circuit of reciprocal favours, gifts, or assistance. The circle can be restricted to two persons or enlarged to include many people. In any case, the exchange is not calculated in monetary terms but is part of a series of acts that maintain and keep up a relationship. The difference from monetary exchanges is marked by the rejection of any form of monetary payment for such favours, gifts, or assistance. In a way, the "payment" is intrinsic to the relation itself, that is the relational good enjoyed by those participating in the relation, and the "money" (not the "currency") is the symbolic medium inherent in the action producing that good (i.e. the reference to the bond). It is in the spheres of society where reciprocity is the foundational norm that good life resides.

The original sin of the lib/lab arrangement lies in the fact that, by ignoring the value and intrinsic norms of social relations inspired by the symbolic exchange, it generates relational evils. The passage from a lib/lab arrangement to a societal arrangement in which morphogenesis is "guided" (steered) requires positive norms (e.g. voluntary work on the part of the healthy, environmental concern) and negative norms (e.g. discouraging prostitution and exploitative uses of labour) that follow a logic of opportunities in which the common good is redefined as a relational good. The reason for this assertion is the fact that a common good without relationality between those who produce it and those who use it renders the ethics of the good life sterile and indifferent. 


\section{The morality of social relations: empirical examples}

The relational vision of society reveals that social problems arise from specific contexts that generate relational evils and that the morality for combating these must be inspired by relational work on these networks.

Let us take the case of social interventions that aim to make young people desist from committing crimes and to reintegrate them into a good society. Various studies demonstrate that friendship groups, intimate relationships, families of formation, employment, and religious communities play a central role in changing the life course of young delinquents. As Weaver and McNeill (2015: 95) suggest, we have to explore "the ethical implications of these findings, suggesting that work to support desistance should extend far beyond the typically individualized concerns of correctional practice and into a deeper and inescapably moral engagement with the reconnection of the individual to social networks that are restorative and allow people to fulfill the reciprocal obligations on which networks and communities depend."

Let us take the case of poverty. As very many studies demonstrate, poverty is not only the product of individual characteristics, but above all of differences in access to opportunities. Social networks are the factor that conditions access to goods and services that can be obtained in markets (Marques 2012). Social inequalities have often been analysed from the point of view of characteristics of individuals or the workings of large opportunity structures such as the job market or the offer of direct income transference policies. In reality, the best solutions to poverty are those inspired by the paradigm of relational work. It is necessary, however, to distinguish between how relational work has been adopted in the United States and how it arose and has been practiced in European countries.

In the United States, relational work is taken to be assistance towards pursuing a life plan in which material help is given to poor or indigent people within a long-term relation that valorises the individual capacities of the poor and unemployed. This way of intervening has some value, but it does not alter the structures that generate social inequality because the goal of escaping poverty is pursued through a personal life plan within the framework of unchanging social structures that correspond to the compromise between the capitalist market and the welfare state (lib/lab), even though welfare measures are performed by third sector agencies (Jindra and Jindra 2015). Relational work, theorised and practiced as the modification of social, cultural, and economic structures, is very different (Folgheraiter 2004; Folgheraiter and Raineri 2012). It aims at helping people who experience life difficulties and vulnerabilities to remodel their relational contexts in a meta-reflexive manner (not directive) so to support their willingness to get a good life through a relational steering. An instructive example concerning relational social work in foster care has been provided by Calcaterra (2017), in which she demonstrates how the relational method provides significant operational guidance to social workers who, to understand how to support 
people, are confronted with important questions regarding conceptual, methodological, and ethical issues, so as to achieve better results in recognising and strengthening the motivation of the same people who are moving towards a desired improvement.

The question is: can we assess the morality of social networks, that is whether and how a network produces moral values such as justice, solidarity, subsidiarity, etc. or, vice versa, injustice, oppression, marginalisation, exploitation, etc.?

The answer can be affirmative, but a relational framework is necessary to understanding this because it involves giving an assessment about relations and the networks of relations, and it is not enough to consider only individuals' intentions (or their "altruism"), or only the morality of the social structures that condition individuals. This is the limitation of several sociological views of the classical welfare state (from Richard Titmuss to Talcott Parsons).

In my view, the morality of a social relation (or network of relations) consists in the fact that it can produce relational goods or relational evils for those who take part in it, independently of agents' intentions.

For instance, (i) the freedom to dismiss an employee can be intentionally good in order to save a company or increase its competitiveness, but it can produce poverty or social inequities (not as a simple "fact," but as a relational evil); (ii) redistributive state policies can have good intentions aimed at assisting the poor, but they can generate a "poverty trap" or other social traps (relational evils). When, how, and why can we say that a social relation is good or bad?

In order to produce relational goods, a dyadic social relation (or the relationality of a network of social relations) should meet the following requisites: (i) a necessary requisite, but not a sufficient one, is that the social relation be good in itself, that is in its own structure or "molecule" and, therefore, in its own elements, which are its goal, means, guiding norm and value pattern), and not only in the feelings, aspirations, or intentions of the subjects/agents; (ii) the social relation should generate an emergent phenomenon that brings a good to each participant; and (iii) the good enjoyed by each participant could not be obtained "otherwise," that is in a way that is lacking the We-relation.

For instance, a "mafia relation" does not meet the first requisite, since its structure is morally bad, although it can meet the other two requisites. On the contrary, a measure of redistribution pursued by public (state) policies can be morally good in itself, but generate relational evils because it does not meet the second and/or the third requisite.

The fact is that social networks are highly ambivalent. They offer opportunities and resources, but also constraints and obstacles to access to and use of opportunities. This can be seen in the research on structural holes and on the brokers that occupy positions of intermediation of information and exchanges between the nodes on networks. According to some authors, 
brokers play a positive role in offering opportunities (Burt 1992). It is argued that the wealth of a society's information depends on the informational potentialities of social circles (structural holes) that social entrepreneurs (bridges) are able to put into contact with one another. According to others, brokers play a decidedly ambivalent role; for instance, Ahuja (2000) claims that structural holes have both positive and negative influences on subsequent innovation. According to still others, they have different functions; for example, the results of the research done by Fleming, Mingo, and Chen (2007) illustrate how collaborative brokerage can aid in the generation of an idea but then hamper its diffusion and use by others.

Certainly, social networks are "assets" (Lazega 2007; Gulati 2007), but we must draw distinctions between the characteristics of each network because the fact of producing relational goods or evils is correlated with the morality of the good life that each network supports. It is important to reiterate that relational goods are goods that consist of relations: they are not material entities, they are not performances, they are not ideas - they are none of these things. They are relations. Let us take two examples, one negative and the other positive.

The negative example is when relational goods are lacking. A very common case, whether in families or in universities and work places, is the presence of structural holes in the networks of relations among people who are managed by brokers who hinder rather than foster communication among all the nodes in the network. The brokers are mediators who prevent people from being able to relate to one another and thus hinder the creation of a relational good. Empirical investigations demonstrate how important the attributes of nodes are in configuring the characteristics of social networks (Wang, Robins, Pattison and Lazega 2015).

The positive example is friendship. Friendship is a social relation that goes beyond individual dispositions. Certainly, friendship flows from people, and only people can be friends and create friendship, which is a virtue for them as persons. But it cannot be an individual undertaking. Ego and Alter are not friends as individuals. Friendship is the acknowledgement of something that does not belong to either of the two, although it is of both of them. This is the relational good. It is the good that exists in common between people; only they can create it, but it does not belong to either of the two people, even if it is of both of them. Likewise, friendship cannot be the product of a social structure; it cannot become an institution, a structure to which people must conform. To be friends, there have to be at least two people who must share and exchange something on an interpersonal level. As Lazega and Pattison (2001) have shown, friendship mitigates the competition in social networks and fosters the creation of social capital. It is the sharing, that is the reciprocal action that generates the We-relation, the relation as the reciprocal action of We, which gives sense and form and content to friendship. Sharing cannot be an explainable fact in individual terms, even if it is not a collective reality: it is not imposed by anyone, it 
cannot be dictated by any authority, and no one can experience it as something constrictive or external. To understand this, it is necessary to move beyond both methodological individualism and methodological holism, which are the two great currents of thought that still dominate the social sciences today. They do not seem to have understood the new realities that are emerging in the worlds of the economy, as well as in those of the production and consumption of goods and services, including the worlds of welfare and the Internet.

In these worlds, we see the spread of productive practices that operate on the basis of a "relational logic" so that the value of goods and services references the quality of the social relations, and not the quantity of the time of the work that was employed to produce them. Social capital is "good" if personal relations are good. In this way, the validity of all the classical economic theories is overturned, theories which, from Ricardo to Marx, computed the value of a good or service in terms of the time necessary to produce it, as lib/lab logic still does to a great extent. This "relational logic" is intrinsically a form of social morality because it involves the fact that a criterion of value is introduced (the quality of the relation) in place of ethically neutral quantitative parameters.

The economy in which the social relation is a value comes to define a new ethics of work and economic transactions. We need to look at things through the lens of relationships rather than just the lens of money. The reason we do that is that behind every financial transaction there is a relationship. It is the relationship that determines the long-term success and impact of what goes on in terms of finance and money. So if one really wants a successful economy, one has to see and give relevance to the social relations that are underneath the financial transactions.

\section{Which eudemonic morality emerges through these phenomena?}

Certainly, the idea of happiness, the good life of individuals as well as of society, depends on the creation of common goods. But, as I have already said, in a complex and globalised society, common goods must be interpreted as relational goods within particular networks that have positive externalities for the surrounding community.

In this regard, it is necessary to consider how the new media (ICTs) are revolutionising "real" (interpersonal and structural) social relations through virtual relations. Clearly, we must distinguish between the different types of media, the different ways of using them, and their specific outcomes. There are media that allow for the production of relational goods and others that generate relational evils. This is what the morphogenetic approach proposes to explain concerning the morphogenesis of the human person, agency, as well as social and cultural structures, in relation to a possible good life.

When people become aware of all of this, social change begins. New processes emerge that are aimed at reassessing relations with others. One 
discovers that working as a team, cooperating with others rather than acting individually, is more effective and satisfying, on condition, obviously, that the task has not been imposed and that teamwork is not a tool used by those in charge to make higher profits. Family bonds are rediscovered as relations that, while being constraints, give a meaning to one's life that other relations cannot give. A growing number of people realise that they can achieve their goals only through new forms of association and new social movements. New demands for justice and social solidarity arise requiring a vision capable of putting the needs and rights of all of a community's members into relation with one another. Indeed, many discover that we are all deeply connected to one another. Each person's decisions, choices, and actions are not purely individual matters but are enacted in relation to others. It is irrational to think of them as simple expressions of the autonomous Self. One comes to realise that, in reality, each person lives in dependence and interdependence on so many others, without whom one could not be the person one is, and could not become the person one desires to become.

It becomes apparent that each individual's history resides in relations with significant others. The human person is not a self-sufficient entity: he/ she is an "individual-in-relation," where the relation is constitutive of the person. We are all in the same boat, in the sense that we depend on one another. And so the question becomes: what kind of boat is this? I think that we can call it: We-relation. But what kind of relation is this? In other words: how should the relationality between us be so that individuals fulfil their own humanity and do not become alienated from themselves to become another individual or something else?

Traditional collective movements - called mass movements - no longer offer adequate answers in that the identity they confer is of an aggregate type and is not relational. The identity acquired by the individual from the fact of belonging to a collective movement based on identification with a symbol (e.g. ecological, anti-global, civil rights movements) can become significant only on two conditions: (i) if it is mediated by an adequate inner (personal) reflexivity and (ii) if the personal reflexivity is capable of realising a relational (social) reflexivity with others. Both of these conditions are hardly ever met by collective movements if they are purely aggregative. They can be present, instead, in collective movements in which people have real relations, and not only virtual ones, with one another, and these relations cause a social form (instituted form) to emerge that is capable of stability and its own action. The social networks on the web (run through information and communication technologies) can do this on the condition - which is by no means a given - that the virtual relations are only a tool, and not a replacement, for intersubjective relations.

In the society of the human, well-being is constituted by the good of the social relation as the path towards obtaining individual goods. The relational good consists in all those relations that can be generated and enjoyed together with others and on which individuals must rely in order to obtain 
everything that they could not have without such a relation. Examples are all of those immaterial, yet real, goods such as cooperation, friendship, recognition, cooperation, solidarity, mutual help, enjoying a positive climate in a firm, classroom, or social street, and so on, which meet most human needs.

\section{The relational character of the moral good}

We have to be clear about what we mean by the relational character of the (moral) good and the good society. Many authors speak of the relational character of the good, but, in reality, they are referring to individual agency. I offer two examples.

Christine Korsgaard observes that the (human) good is, above all, an affirmation that something is normative for me, for my condition, as an act of sympathy with myself. ${ }^{1}$ She claims the relational nature of the good, but she does it from an individualistic and, in the end, constructivist point of view.

In adhering to Kant's philosophy, she maintains that the good has a relational character in as much as a certain entity becomes held in common, that is it is "constructed" as being shared by rational subjects who are capable of having sympathy with themselves and living this sympathy through empathy with others. The good is relational in that it is constructed with ends that are "shared among all of us" ("Good, then, is the schematic name for the solution to the problem of shared ends. This, then, is my answer to the question of why we operate with the concept of the good: because as rational creatures who are capable of seeing the world through the eyes of others, we are faced with the task of constructing a state of affairs that is, as far as possible, good-for us all," Korsgaard 2013: 24-25, italics mine).

For Korsgaard, then, the good is relational, not because it consists of "good" relations, but because it is shared by individuals who use their relations to make something good held in common. She does not see the relational constitution of these common goods, because the goods do not consist of relations properly. In short, relations have no substantive reality in themselves. The good life does not require a reality endowed with certain relational qualities and properties in itself, but is good in that it is constructed as being good for each of the participants.

Ana Marta González (2011), reflecting on these issues, identifies some paradoxes and internal contradictions in the thought of Kant that can be

1 "For to say that something is good-for me is to describe something's relation to my condition as having normative implications, and that in turn is to endorse the view of myself that, simply as a conscious being - as a being who is in her own keeping - I necessarily take of my own condition. One might see the endorsement of that view as an act of sympathy with myself." (Korsgaard 2013: 24, emphasis mine). 
traced to a lack of a relational vision. First, while Kant takes the educational process to be a radically moral enterprise all the way through - and hence, placed in a relational context - he also aspires to constitute education as a science, to be improved through experiments, thereby paving the way for a systemic approach to education; in spite of its moral inspiration, his systemic approach not only could enter into conflict with the moral demand of taking each individual subject as an end, but is also marked by an intrinsic paradox, already involved in the ambiguity of the term "humanity," which might mean (i) humanity as a moral disposition present in each individual human being, or (ii) humanity as a whole, as the "human species" (ibid: 433). Secondly, González finds that the Kantian conception, "leaving aside the mechanism of education to focus on its relational dimension," leads to the affirmation that "the attainment of a moral culture depends on teaching children to act upon principles, and hence autonomously," with the paradoxical consequence that "in order to educate autonomous human agents, we have to engage in a process marked by heteronomy" (ibid: 442).

González' critique of Kant's philosophical conception regarding education, claiming a relational approach, is enlightening and correct. "While improving education may become the object of a systemic action, education is always the object of a moral relationship" (ibid: 437); “... moral education, as something directly linked to personality, is always beyond the reach of those techniques, and dependent on a relational approach to education" (ibid: 438). This relational approach, however, remains focused on the promotion of the individual human being as the way to get better relations. The structure of education as a social relation is basically referred back to the moral quality of individual people: "autonomy is prepared in the context of personal relationships, interested not so much in the cold development of potentialities as in the real good of the person" (ibid: 452).

If, as González acknowledges, educating means performing a relational activity, then we have to understand education as a sui generis social relation, as a dynamic structure in itself, situated in a relational context, and act with it and on it, not only on individuals. Otherwise education remains only the object of individual agents' morality, and not also a moral entity in itself, with its own qualities and causal properties. Interpersonal relations are considered as "conductors" of individual morality, not yet as moral goods (or evils) in themselves. To become truly relational, the moral good requires the adoption of the second person perspective on the part of the agents, i.e. a dialogue (a dialogical self), and not only a first-person perspective. The latter, of course, is absolutely necessary, but it is not sufficient in order to get "good relations," which is what Kant believed.

\section{Summary: the good life through social relations}

Ethical neutrality in social theorisation is basically a myth. Certainly, sociology distinguishes itself from social theory because, in analysing social 
matters, it does not have to take sides on this or that value. It is inevitable, however, that it, too, always presupposes value choices (Lidz 1981), which obviously cannot be those of direct ethical or political engagement; otherwise, sociology is transformed into an ethical or political doctrine. The moral burden of explicitly declaring the value choices in play is incumbent on the sociologist when entering the arena of social theory, where such choices can obviously be diverse and plural.

As for my relational sociology, it makes reference to a social theory that does not make a priori value choices, but points to the good or the bad in the effects produced by the societal dynamic. It juxtaposes the ways in which acting subjects generate different social consequences, which can contradict not only their situational expectations, but also the values of the collective morality that they support.

Relational analysis leads one to conclude that postmodern morality erodes the common good because, consciously or, more often, unconsciously, it erodes social relations. There are obviously good reasons for assessing social relations negatively when they are used to exploit people (such as in human trafficking or prostitution), to organise social groups for the purposes of common criminality or corruption, or for other morally negative ends. On this basis, however, the postmodern morphogenetic society has elaborated a social morality according to which the good life consists in an indefinite increase in life opportunities, on the assumption that human identity can continuously change, endlessly altering its social relations. Today's moral norm dictates the celebration of "relationism" as the path towards the individualisation of the individual. In the postmodern cultural system, it is assumed that happiness consists in this process.

Reality ends up debunking this morality. The idea that in order to achieve a good life the logic of opportunities must be untied from the value and norms of social relations leads to continual failures. The reason lies in a precise sociological reality. In fact, happiness is sought in the creation of ever new social relations; it becomes possible to enjoy all possible opportunities only on condition of immunising oneself from the relations themselves, that is, on condition of not rendering any particular relation (any opportunity) necessary: thus, on condition of not binding oneself to anything or anyone, if not for the opportunities of the moment (this is the "pure relation" theorised by Giddens).

The moral norm celebrated by the unbound morphogenesis emerging from the crisis of the lib/lab system makes the maximum contingentism and relationism imperative. It celebrates relationality while negating it at the same time. But negating the identity of the relation means also negating the identity of the subject: hence, the impossibility for the individual of achieving authenticity in his/her identity. Such is the paradoxical outcome of this conception of the good life: living in relations without tying oneself to them. In this way, the individual can increase his/her life opportunities always on condition of not privileging any one social relation over others, 
which - according to this collective narrative - would involve limitations and discriminations. It is a morality of nondistinction because the moral norm dictates that no distinction should be made, since every distinction is discrimination. But in this way, a "reverse discrimination" operates because one chooses to not choose (one decides not to distinguish). This is a moral norm that leads to cultural and moral regression because human civilisation requires the continuously renewed and creative use of distinctions.

Considering everything and every human action within the relation in which we find it and looking at it from this point of view is essential to giving meaning to things and actions. Human life in pursuit of happiness - in a couple, in a working relation, or in the search for a job that isn't there - does not mean alienating people within the limits of the relational situation in which they find themselves, but the complete opposite. It means giving them a perspective for managing their human condition in a horizon of openness to meaning - openness to other relational worlds, that is.

We must acknowledge that still today we lack a proper reflexivity on the relational nature of the good life if we understand this expression in the sense that the good is constituted by certain social relations instead of others. These are the relational goods that bring truly human happiness going well beyond material welfare. 


\section{The emergence of new social subjects generating relational goods}

\section{Identity and relationship: "distinguish to unite"}

The topic of the relational constitution of identity pertains to every existing entity. It will be addressed herein only as regards the human person (as social agent and actor) and those social formations in which the person identifies herself as belonging to a collective (We-relation).

Identity and relation are two distinct concepts, but their distinction does not signify separation as they are ontologically and phenomenologically connected. The theoretical and practical problem is to understand how their connection is articulated. The two perspectives of identity and relationship cannot be separated empirically, but only analytically. The human person, as an "in-Self" being, does not find her identity by remaining in herself, but can find it only by relating to the Other-than-Self. At the same time, this relation cannot disregard the identity of the terms that constitute it. In synthesis, identity is formed in the relation between the Self and the Other-thanSelf, without the Self's and/or the Other's nature (substantia) being entirely absorbed by their relation.

It is possible to understand this reality if we are able to see the enigma of the relation, which consists in the fact that the relation unites the terms that it connects (it creates commonality) while, at the same time, it respects and promotes their specific difference. This potentiality is due to the fact that the relationship that binds the subjects is not only a bond (a structural link, a religo), but is also a subjective reference (refero) that modifies the structural bond generating an emerging effect. Only in this way does one understand, for example how the identity of a couple, a family, a civil association, a sports team, or an orchestra is that of a We (We-relation) in which each member can and must find his/her own collective identity without negating or demeaning his/her own personal identity.

As human persons, we naturally ask ourselves what our most meaningful identity is, the one that substantiates the reasons for our life beyond the contingent identities that we assume over the course of our existence (the identity that we have in our family of origin, in school, at work, in civilian, political, and religious life). The response to this question ("Who am I and 
why am I alive?") cannot be given by the person only with reference to herself, but must be found by relating to something or someone that can give one the sought after identity, in which it is possible to find the ultimate and most satisfying meaning for one's existence.

Obviously, we can give ourselves many different answers. When we succeed in putting aside all contingent historical identities, we find this deeper identity in the relation with an ultimate reality (the totally Other), in whichever way the latter is understood. This consideration, which is a statement of fact corroborated by empirical research, conveys how essential it is to understand identity as a relational reality. For those who have a religious belief, in particular, the deepest and ultimate identity is given by the relation of divine filiation, by feeling that one is a child of God.

Having already dealt extensively with the concept of relationship, I will first focus on the concept of identity and then I will try to understand the constitution of the human subject as an emergent effect of personal and relational reflexivity, and the challenges that this constitution must face in the process of social morphogenesis.

\section{The concept of identity}

The concept of identity is polyvalent and poses a series of problems. In the field of philosophy, the question of identity assumes a problematic form because we have two models of identity available: idem identity (the selfsame) and ipse identity (the self in its existence). I propose a relational reading of these models. Idem is the identity of something that endures over time while appearances or, as the Aristotelians say, "accidents" change. Since antiquity, its philosophical model has been substance. Substance is the substrate, the suppositum, the support, which is identical in the sense that it is constant, and in its sameness does not change: it does not depend on historical time even if it passes through the vicissitudes of time and can learn from them.

Ipse, instead, consists of the identity of a Self that rediscovers itself (it reidentifies with itself reflexively) when confronted by the observation of changes in itself due to the variability of feelings, inclinations, desires, and positions taken as situations and historical circumstances evolve. Ricoeur (2004) gives the example of the ipse identity of the individual who keeps a promise. Ego (idem) keeps his promise despite the changes to his internal state, for example, of mood (ipse). I am the same, and I conserve myself as the same person despite having changed over time in certain internal or external aspects (at 70 years of age, I am still Me, the same as when I was 10 years old, even if I am obviously a bit changed). However, Ricoeur does not see the social contexts in which idem and ipse are relationally constituted.

In the field of the human and social sciences, the problem of the person's identity exists in relation to two types of different questions. One refers to the perspective of the first person, the other to the perspective of the second person. 
1 When the question is "Who am I for myself? Who do I want to be for myself?" the answer is produced by the internal conversation, that of the individual who reflects within him/herself on the social context. We are speaking about the individual identity that arises - as Archer (2003) maintains - as an answer to the regular exercise of the mental ability, shared by all (normal) people, to consider themselves in relation to the (social) context, and vice versa, considering that "each mode of reflexivity is a distinctive way of deliberating about oneself in relation to one's society" (Archer 2003: 349).

On this level, the social relation is an attribute of the Self with reference to the social roles one plays in a contingent context (in the family, at work, in associations, etc.). Personal identity is individual because it refers to the ultimate concerns of the person, which are selected, deliberated, and pursued in the intimate space of individual self-consciousness in response to the context. The tenet according to which the person "is what she cares about" should be interpreted in a phenomenological sense, that is in the sense that the person becomes what she loves by changing her ultimate concerns and what follows.

2 When the question is "Who am I for the (relevant) others? What do I want to be for them?," the answer is social identity. The others can be family members, relatives, close friends, work colleagues, members of an association or community, and so on. Social identity can be a $M e, W e, Y o u$ in the way that Archer (2003) has well clarified. It can be interpersonal, when it is referred to another person (e.g. I am the son of Mary), or collective, when it is referred to an impersonal social category or group (e.g. I am a teacher, an Italian, etc.). When the identity attributed to Ego by others is elaborated by Ego in his/her mind (personal reflexivity), the resulting personal identity is still of an individual type, because it is referred to Ego as an individual who can accept, negotiate, or reject it in different ways.

Identity based on relational reflexivity has a different nature from that which people elaborate within themselves and for themselves because relational identity answers to the ethic of the second person and not to that of the first person. The social identity becomes relational when Ego identifies him/ herself in that identity by exercising his/her own reflexivity on a relation that includes the point of view of the Other, and on this basis, it redefines its identity differently from how the Ego would have elaborated it in the first person. The term "Relational Self" here means the capacity to consider one's actions in light of others' needs and feelings (Gelfand et al. 2006: 431).

From interviews with young university students, Archer (2007) draws the conclusion that young people make their life choices by essentially resorting to their inner conversation. For her, resort to their internal conversations 
alone can establish their own portfolio of engagements and finally lead to the endorsement of a course of action resulting in a (fallible) project for its realisation. At the same time, with great insight, she analyzes how young people, in making decisions, can be influenced by the dominant modality of reflexivity they have learned in the family of origin as well as by the choice of the working sector which traces the dominant modality of their reflexivity. I believe that, without denying the importance of people's inner conversation, we need to take a more properly relational perspective. In other words, young people, like all human persons in the course of their existence, make decisions not only by reflecting on their own preferences, but rather by having to take into account the relationships with the significant others that are present in the context in which the decision must be taken and assessing the consequences of their decisions on these relationships. The decisions of young people, as well as of people of different ages, have to balance their own inner desires and projects ("I'm keeping my options open") with the value attributed to primary relationships (Me) and secondary relations (We) in which they are embedded. These relationships have a decisive impact on the social role they have to play, regardless of the awareness of them that they have individually. I call relational reflexivity the need to respond to relations with a You and with significant Other. It is distinct from the individual reflexivity of the inner conversation. Take the case of a young person who prefers a certain professional occupation to another or quits a job because it gives priority to the relationship with the partner. This decision does not depend so much on the reflexive mode learned in the family of origin, but rather on how the person evaluates the possible outcomes of her decision on interpersonal relationship (I-You) with the partner, and what responsibility she chooses to assume for these results, redefining her concerns if necessary.

Archer denies that

a couple can succeed in forging a life together ('our' life as a couple after graduation) on the basis of hermeneutically entering into one another's aspirations for what kind of life that would be, given appropriate detachment on the part of both. Empirically - and assuming they are in love - it is quite likely that their external conversations together will cover this ground to the best of their abilities. But these discussions cannot be a necessary condition for the source of 'We-ness.' The best of their abilities is not good enough because as subjects they cannot avoid the double hermeneutic. However hard the two try, they produce Alter's interpretations of Ego's self-interpretations and vice versa, doing so seriatim with no way out of this trap.

(Archer 2019b: xii) 
I think that Archer's thesis about the impossibility of escaping from the double hermeneutics is correct when it happens that Ego and Alter are not able to activate their relational reflexivity, but this is not always the case. In the couples who create relational goods, Ego and Alter are capable to grasp the meaning of their different aspirations and the possibilities to reconcile them through the cognitive ability inherent to empathy (understood as Stein 1989 does, differently from Husserl), and thus find the way to create a sharing of feelings and practical goals that lead to build a We-ness. The thesis of the impossibility of escaping from the double hermeneutics cannot be generalised.

In short, a first-person perspective is not enough to define the whole identity of the human person. Second- and third-person perspectives are also needed, as they are constitutive of people's social identity which affects their personal identity. Without an ability to take a second-person perspective and also a third-person perspective, humans are not able to think morally.

In other words, relational identity is the result of an exercise of reflexivity carried out on the relationship as such, including in it the actions and expectations of the Other, in order to redefine one's identity in that relation, by appreciating that relation as a good, renegotiating it or rejecting it as negative or indifferent for some reason.

An example can be the identity that a mother must elaborate, not as an individual who has her own concerns, thoughts, and feelings in respect to the son, but having worked out her identity as a mother, considering how her son sees her and desires her, what he expects of her, how he wants her to be for him (the same thing, of course, applies to the father). We know that it is the child who generates the identity of father and mother. That is why a mother and a father must take into account the fact that their parenting relationship has consequences on the child that do not necessarily correspond to their individual concerns, thoughts, and desires, as well as considering the fact that any parental relationship depends on how the entire network of family relations operates.

In the ethic of the second person, the subject defines his/her identity reflexively starting from the question: "How do You consider me?," "How do You want me?," "How must I be for You?," where the You is not the "I" that questions him/herself, but is an Alter who desires a certain relation with Ego. By accepting the relation with the Other as being significant for the Self-identity, Ego is called upon to accept the You and to acknowledge the Self-identity as an Other, which entails a new identity (Ricoeur 1954), that is it is a reidentification that explains the changes in the person.

To clarify the point, let us consider difference between the first- and second-person perspectives. According to Lynne Rudder Baker, the first-person perspective is a complex mental property that enables one to conceive of one's body and mental states as one's own. She writes (Baker 2015: 79-80): 
A first-person perspective is a primary-kind property that members of the kind person have essentially. A first-person perspective is a dispositional property with two stages - rudimentary and robust (...) One acquires a robust first-person perspective as she acquires a natural language. To conceive of oneself as oneself in the first-person, one needs a self-concept - a nonqualitative way to think about oneself. I take concepts to be inextricably linked to language. There's no self-concept, and hence no robust first-person perspective, outside a language. Learning a language is learning concepts, and learning concepts is learning about reality. After a child acquires a battery of concepts like water, mommy, ball, want, toy, chair and so on, she is ready to understand a concept like mine, which she uses to apply to what belongs to her - conceived of as herself, from the first-person perspective. A robust first-person perspective - an ability, not just to recognize oneself as distinct from everything else, but to conceive of oneself as oneself in the first person - makes possible almost all our characteristic human activities.

So far, so good. The second-person perspective is quite different. It consists in the fact that Ego considers the Other as him/herself and reflects on the fact that his/her own identity, as well as that of the Other, depends on their relationship. It presupposes a reflexivity on the relationship that is not conducted from the point of view of the "I," but from the point of view of the Other with which Ego is in relation, and, as a consequence of this, by taking the point of view of the relationship itself.

The ethic of the second person is not the assumption of the Other in me, as some believe, but it is the ethics of my relationship to the Other considering the fact that the relationship is not the product of my own subjectivity, just as it is not the product of the subjectivity of the Other (i.e. the product of two perspectives in the first person), but it is ethics related to the good of the relationship itself as individual goods depend on it, that is to the relational good.

The difference between the perspective of the first and second person marks the difference in kind between the inner reflexivity that underlies the subject's individual identity and the relational reflexivity that underlies the relational identity of the subject. To understand this difference, it can be instructive to make a parallel with individual and social responsibility. For example, some parents refuse the vaccination of children, claiming that they are harmful to them. In making the decision to vaccinate one's child or not, the parent can reflect individually, based on his knowledge and opinions in relation to his context, and then uses personal reflexivity. If instead he considers the fact that if he does not vaccinate his child he puts other children with whom he plays at risk, then he uses relational reflexivity, because he takes the point of view of the relationships between his child and other children from which relational goods or evils can come. 
Without relational reflexivity there is not really a dialogue; there is no true "encounter" with the Other, because identity remains self-centred, if it relies only on individual reflexivity (Caetano 2015: 68). There is not even a deep knowledge of the world because knowledge is relational (Fiske and Haslam 1996). And there is no adequate socialisation because a good socialisation is the outcome of relational processes in which the reference to the Other is fundamental in order to establish a shared identity (Van de Walle 2011). The well-being of the person is essentially relational (White 2017).

The notion of relational identity is delicate and complex because it highlights not only the reflexive cognitive components of the mind, but also the feelings and the cultural models of reference of the subject. On the capacities of cognitive reflexivity I will say later, here I limit myself to mentioning the affective-emotional and cultural dimensions.

There is something sensible in the argument by Jan Burkitt (2016) according to which we need an understanding of agents as interactants, ones who are interdependent, vulnerable, intermittently reflexive, possessors of capacities that can only be practised in joint actions, and capable of sensitive responses to others and the situations of interaction. But I would not fully agree with his view that "instead of agency resting on the reflexive monitoring of action or the reflexive deliberation on structurally defined choices, agency emerges from our emotional relatedness to others as social relations unfold across time and space" (ibid: 323 ). Emotions are certainly one component of social relations, as is affection in its various forms (Scheve (von) 2018). Ana Marta González (2017) has clearly highlighted the role of emotions as sites of social reflexivity. However, in contrast to Burkitt, while accepting that feelings are relational commentaries upon one's concerns and ideals, I think that they are not sufficient to support one's relational identity when the latter faces serious challenges. Since emotions are relationally constituted within cultural worlds (Hong 2004), they need a structured social context to support personal and relational identity.

As regards the cultural dimensions of reflexivity, both kinds of questions on identity (who I am for myself, and who I am for others) have to do with those value patterns that orient the ideals and images (representations) of oneself. When a person asks these questions, she must find the answers in symbolic references, usually linguistic, to define herself in her own identity and for the others. In asking: "In what do I identify myself?," "What is the model of life that I like?," the answer is a cultural identity. The cultural identity responds to the question about the symbolic world to which I feel a belonging or a reference, in terms of beliefs and values in a modus vivendi or in a lifestyle that willy-nilly associates me to others and is generative of meaning for my Self. This symbolic world is made up of people or things that have value for me. It is identity in the sense of configuring one's image in relation to (by belonging to) other persons or certain sociocultural groups, or even things (e.g. in pantheistic religions). This relation includes cultural aspects - that is framing rules and feeling rules - based on which 
individuals validate their own moral action and feelings and are, at the same time, evaluated by others (Stets and Carter 2012).

Personal identity becomes problematic when the answers to the aforementioned questions are multiple (the Self perceives him/herself as a $\mathrm{mul}$ tiple self), that is, in situations in which the "I" identifies or must relate to different identities and must try to keep them together while making choices, or risk fragmentation, alienation, and other pathologies (Gambino 2017). One can agree with Harrison White when he claims that the problem of identity underlies a control problem. But White's approach is strongly reductive and, in some respects, misleading.

White, who is considered by many scholars as the first theorist of relational sociology, defines identity as "any source of action not explicable from biophysical regularities, and to which observers can attribute meaning" (White 1992: 6). So far nothing wrong. The sore point lies in the fact that the constitution of identity is attributed to the social network, putting the agency in the background, that is making it fully dependent on existing social links. In Identity and Control, White makes the claims that agency is "the dynamic face of networks," that "stories describe the ties in networks" and that "a social network is a network of meanings" (White 2008: 65-67, $245,315)$. His argument is that identities emerge as a result of interaction in the social molecules (White 1992: 22) and "spring up out of efforts at control in turbulent contexts" (White 2008: 1).

Certainly, nobody can deny that identities try to control themselves in relation to the environment, which is made up mainly of other identities. But the existence and operation of identity cannot be reduced to a control problem. First, the need for control is only one of the components of identity (it is just one of the modes of the normative component of the relation). Second, by claiming that the starting point of sociological analysis should be identity rather than persons, given the assumption that persons can never be analysed separately from their environment, White (1992: 24) drastically reduces the complexity of the relationship between the human person and her identity. Third, he commits an ecological fallacy ${ }^{1}$ when he states that identities are tagged to individual human beings (White 2008: 10) by arguing that identity is a product of the network of social interactions; for him, persons come into "existence and are formed as the result of overlaps among identities from distinct network populations" (2008: 129). Fourth, he claims that identities are constituted by an essential relation - the tie that is constitutive of identities (White 2008: 20-36), and therefore conceives the constitutive relationship of identity essentially as a bond (religo), which means to ignore the intentional and value reference dimension (refero). In

1 An ecological fallacy (or ecological inference fallacy) is a formal fallacy in the interpretation of statistical data where information about the nature of individuals are deduced with operations of inference from the group to which those individuals belong. 
short, notions such as network and tie reflect a relational stance, but they are interpreted from a structuralist point of view. For these reasons, the so-called relational sociology proposed by White is strongly deficient. The same applies to the sociologies of those who follow Harrison White, such as Jan Fuhse and Reza Azarian.

\section{How to connect identity and relationship?}

The connection between identity and social relations is an inter-dependence and an inter-penetration between them. One term depends on the other in such a way that something of identity enters into the relation, and the relation goes to constitute a part of identity. Both personal and collective identities are formed within an environment constituted by a structural and cultural domain. The formation of identity requires a symbolic system of reference within which the generative mechanism of refero-religo-emergence of identity is carried out. It is possible to distinguish the contribution of the individual subjects and the autonomous contribution of their relationship (Tam 1989). Individual virtues (outcome of individual reflexivity) can generate social virtues (i.e. virtuous relations such as trust, friendship, cooperation, loyalty, and forgiveness) at the condition that the subjects are able to exercise an adequate relational reflexivity on the network of their relations. Personal identity problems and relational evils are significantly correlated with the fact that the social networks in which people live are deficient in supporting the conditions and opportunities to exercise their relational reflexivity.

Saying that the identity of Ego is constituted in relation to an Alter means affirming that there exists a relation between Ego and Alter to which they give their personal contribution, but which takes on its own autonomy, unpredictability, and spontaneity to the point of becoming a resource or a challenge for Ego and Alter. To "ex-ist" (from the Latin ex-sisterre) means "being outside" the terms of the relationship. For example, saying that Mark is a responsible professional in terms of his relational identity means indicating that he cares for the relationships with his customers in such a way as to derive his professional identity by treating such relationships as an autonomous reality and not as an expression of his inner identity.

An issue sometimes raised has to do with the question of whether, from the ontological and epistemological point of view, identity precedes the relation or vice versa (who "comes first"?). Martin Buber's affirmation according to which "in the beginning is relation" (Buber 1970: 68) has a theological and metaphysical significance that gives the relation absolute primacy (Metcalfe and Game 2012) as "the living center" of community (Avnon 1993). In a different vein, from the sociological point of view, the statement that is at the base of my relational sociology - "in the beginning is relation" (Donati 2011a: 17-25) - should be understood in the sense that the individual's action is already always situated within a social context of pre-existing 
relations and must be analysed and understood in that relational context, with its history and its dynamics. This should be done without dissolving identity in the relation or vice versa, because substance and relation are $c o$ principles of reality. Identity is not entirely given by the relation, nor does the relation derive immediately from identity. If we reduce the identity to the relation, we fall into some sort of relationism, which dissolves identity in an indefinite referencing of relations to other relations (relativistic pragmatism); if we take the second position, we fall into a sort of fundamentalism, because identity comes to be somehow "reified" and quite often used as a "weapon" for opposing other identities.

\section{Overcoming unrelated thought with relational thinking}

Unrelated thought refers to those ways of thinking about identity that attribute qualities to entities exclusively on the basis of their substantial nature without making reference to the relational context (situation, circumstance, environment) in which they are situated and are subject to change. This way of thinking, which spans the history of Western culture, considers social relations as "accidents" which, although real, do not alter an entity's identity. In contrast, "relational thinking" attributes qualities and causal properties to entities taking into account their own nature but at the same time considering their actual identity as a relational reality that emerges from the dynamics of a relational context.

To understand the movement from one way of thinking to the other, it is useful to distinguish between three types of semantics in defining identity (Table 8.1).

Table 8.1 Three ways of defining identity

\begin{tabular}{|c|c|c|}
\hline $\begin{array}{c}\text { Identity through } \\
\text { self-reference }\end{array}$ & $\begin{array}{c}\text { Identity through } \\
\text { negation }\end{array}$ & $\begin{array}{c}\text { Identity through } \\
\text { relational differentiation }\end{array}$ \\
\hline $\begin{array}{c}\text { A's identity is given by a } \\
\text { simple relation of equality } \\
\text { or equivalence with itself } \\
\text { (according to a self- } \\
\text { referential symbolic code } \\
\text { of identity) }\end{array}$ & $\begin{array}{c}\text { A's identity is given by a } \\
\text { relation of negation of } \\
\text { what A is not (it is a } \\
\text { double negation, according } \\
\text { to a binary code) } \\
\text { (it ends up evaporating) }\end{array}$ & $\begin{array}{c}\text { A's identity is given by a } \\
\text { relation (reciprocal action) } \\
\text { with the Other (the not-A) } \\
\text { that generates A's identity } \\
\text { as an emergent effect } \\
\text { (according to a generative } \\
\text { code) }\end{array}$ \\
$\begin{array}{c}\text { A = not (not-A) } \\
\text { (e.g. John is simply himself) }\end{array}$ & $\begin{array}{c}\text { (e.g. John is not Mark or } \\
\text { anyone else; but, } \\
\text { radicalising the distinction, } \\
\text { John is not John) }\end{array}$ & $\begin{array}{c}\text { (e.g. John is himself in } \\
\text { relation to Mark and } \\
\text { everyone else, even with } \\
\text { respect to himself in the } \\
\text { reflexive process of } \\
\text { distinguishing I, Me, You) }\end{array}$ \\
\hline
\end{tabular}

Source: Author. 
In the monistic conception, typical of ancient classical thought, identity is understood as substance, as something that does not need to put itself in relation to something other than itself. Identity is founded on the principle of self-reference $[\mathrm{A}=\mathrm{A}]$, by which A's identity is immediate and exists without mediations. From a social historical point of view, talking about the general population, the individual's identity coincides with that of the social group of belonging (tribe, social stratum, local culture) and is lived in an almost automatic way through the internalisation of habitus. The space of personal reflexivity is limited, and relational reflexivity, even where it can be practised, is not theorised.

This cultural semantics obviously does not negate the existence and importance of relations in defining social identities, but it considers them as something natural and taken for granted (e.g. the identities that come from family relations and those belonging to a tribe, gens, ethnicity). From the political point of view, all social subjects (and civil society itself) tend to be identified with the attribution given them by the community or the state (polis, civitas). This way of thinking about and experiencing identity is typical of societies characterised by segmentary differentiation (tribal and ancient societies) and vertical differentiation (i.e. stratified by class, such as medieval society).

The dualistic conception of identity, instead, is typically modern and is based on the principle of difference, by which [A = not (not-A)]. In other words, A's identity is given by the negation of all that is not A. Identity is formed on the basis of difference understood and managed in a Hegelian dialectical way or as a total separation according to a binary code $(0 / 1)$. For example, civil society is that which does not identify with the state, and thus its identity is constituted by the negation of the political society. In this semantics, relations certainly become important and, above all, in potential continuous change as compared to what happens in the monistic paradigm. But curiously enough, in its evolution, this semantics is radicalised to the point of leading to negation of the same operation of negation, that is it leads to making all the distinctions indistinct (as happens in Latour 1999). So it ends up evaporating (as succinctly indicated in Table 8.1).

The relational conception of identity defines difference not as opposition or separation, but as an emergent effect of reciprocal actions between A and non-A (i.e. as a relation). Identity is founded on the relational principle by which $[\mathrm{A}=\mathrm{R}(\mathrm{A}$, not-A)]: that is, A's identity is given by a relation (R) between $\mathrm{A}$ and not-A, where the relation becomes binary only in very special cases. In this semantics, identity is defined not by binary dialectic negation, but rather by relating to an otherness through exchanges, communications, and transactions. In short, to say that the identity of Ego is different from that of Alter means that they have a relation in which they share something at the same time that they differ in other aspects. For example, Mario's Italian identity is different from John's English in that they have two different citizenships, but they are both citizens of a state. If we conceive the difference as a relation, we can see how that difference unites them 
while respecting and promoting their difference (this is what the enigma of the relation consists of). The identity of civil society, for example, is defined in relation to the otherness of the state and on the basis of this relation, which entails a distance but does not entail negation of a dialectical type, rather it implies exchanges and interdependencies.

Table 8.1 gives a very concise example of the differences between the three ways of defining identity. While in monistic semantics John is simply himself, in binary dialectic semantics John is neither Mark nor any other person with whom he can be compared; but, when the negation is radicalised, John is no longer distinguishable from himself. In the third semantics, John's identity consists in being in relation to Mark and all significant others, and also in relation to himself when he reflects on the distinction between I, Me, You as an acting subject.

Willis Overton (2014) offers us an example of relational differentiation when he writes,

[T] he prevailing Cartesian split narrative was that mother and infant constituted two separate independent entities, and that the glue that came to bind them together was reinforcement via the feeding situation. The alternative narrative offered, especially by Bowlby and other's known as 'object relations theorists' such as Donald Winnicott, was relational in nature, arguing that mother and infant formed an initial psychological unity and the question was not how two separate entities become glued together, but how a single psychological unity differentiates into a relational two. Harlow's work severely weakened the Cartesian narrative, and the work of Bowlby and his coworkers demonstrated the several phases or stages in the relational differentiation of the mother-infant matrix.

(Overtone 2014, 329-330, emphasis mine)

Relational semantics $[\mathrm{A}=\mathrm{R}(\mathrm{A}$, not-A)] implies that Ego defines his/her identity by taking a certain distance from him/herself to make room for comparison with the Other, which means recognising that there is a certain contingency in the very unity of personal identity, which therefore comes to be constructed in a complex way, through Ego's relational reflexivity urged and favoured by what is other than oneself.

Postmodern society should take into account the necessity of redefining identity by relating to others no longer in a functionalist manner, but rather in a suprafunctional one. The "I" is formed by relating with the Other and through the Other than the self. The "I" and the Other must no longer become specialised functionally (and thus be distinguished by the separation of tasks and identities), but, vice versa, must find their own identity in a certain way of being in relation to the Other, sharing some things and distinguishing themselves for other things.

In the dualistic (i.e. dialectic or binary) semantics, the distinction is a division - a slash - for which one is on one side or the other; in the relational 
semantics, the distinction is a relation that differentiate the terms starting from something they share. Both personal identity and social identity are relational, although in different ways.

The ontological reason that substantiates this statement lies in the fact that any difference arises from what is common rather than vice versa. In this regard, it may be useful to consider Heidegger's thinking according to which "human beings are always already social and dwell originarily in a social world" (Aspers and Kohl 2013: 487). This statement should be read as Heidegger's comment on Aristotle. Following this argument, Knudsen (2019: 337) claims that "in general, Heidegger's ontology proposes to understand being through a unitary, relational structure between self, others, and world." Personally, I appreciate these efforts to read Heidegger in a relational key. But I think that the tension between social relationality and self-relationality central to Heidegger's ontology of Dasein is not really resolved by him because he does not have a theory of the relationship. He does not articulate the social relation in any way, and certainly not in the sense of Emirbayer's pragmatism as suggested by Aspers and Kohl. However, I can recognise that there is an opening of Heidegger to relationality.

In my view, Heidegger's ontology can have some interesting relational developments provided one gives it a certain interpretation. In his Letter on Humanism, Heidegger (1999: 287) writes, "In Sein und Zeit, it is with intention and due to prudence that it is said il y a l'être: being «gives itself». The expression il y a does not exactly translate as «it is given» (es gibt) because the «it» $(e s)$ that here «gives» (gibt) is being itself. It «gives itself» (gibt) indicates the essence of being that gives, granting it its truth. The giving of itself to openness, together with the openness itself, is being itself."

I believe that it is possible to give an interpretation of Heidegger's thought that allows us to see both its limits and its possible developments in light of the relational approach. In fact, saying that "being gives itself" means that being is in itself "free giving," that it has the qualities and properties of "givingness," and, in this sense, being is not only open to the relation, but it is intrinsically relational. If being, as Heidegger says, is "the giving of itself to openness, together with the openness itself," I wonder: maybe Heidegger is not speaking in this definition about being as relationality? But Heidegger does not speak of relationality. Being for him is that which simply "is," as if being (its truth) could not become "other than that which is given."

Instead, for my relational sociology, if being is an act, or better, if what exists has its own "act of being" (the "act of being" endowed with its energeia that pertains to what exists), and this act is relation, precisely due to this, that is its intrinsic relationality, being is not static (it is not morphostatic), but is dynamic and morphogenetic. This means that it can develop (it can "progress," i.e. it can move forward or onward over time and space). Therefore, this is my argument, being permits its own transformation on the sole condition that its intrinsic relationality is respected. Against this 
background, it is possible to speak of relational essentialism for human and non-human beings ${ }^{2}$ Human essence has its own historicity, which means that we cannot completely separate substance and accident once and for all, because there are real accidents (such as relationships) that constitute the substance so that it can develop over time.

It is in light of this ontological consideration that, in my opinion, we should interpret Heidegger's perspective in Identity and Difference (1969), according to which identity is not equality or equivalence. He observes that, to make the difference, two terms are necessary, in order to have equality only one suffices. ${ }^{3}$ We can thus say that, while in the idem, difference disappears, in the ipse, difference manifests itself. The relation of the human with its being is characterised by identity and not by equality. What is human and its being co-belong to each other because they remain different although they share an identity consisting precisely in this co-belonging. Without the one, therefore, the other is not given, even if the identity of one is not that of the other if not in the relationship of mutual belonging.

I call this founding relationship "vital relationship." This relation to oneself is a renewed belonging to oneself through a relational feedback with the Other. It is a belonging to oneself (Egol) because, as a relationship, it implies the passage from a non-being to being someone different (Ego2) (someone, not something) in a reference system (refero) that keeps me bound to Other than myself (religo). The "non-being" is a possibility, usually undetermined, that should prove to be compatible with the transition from Egol to Ego2.

In other words, it is an emerging relationship (creative) that makes me exist unlike in the past on the basis of another reference system that I have chosen and to which I am bound, even if only temporarily. The I cannot say to him/herself "I am myself" except through the Other than self, which may be a physical entity or another person in the world, in all orders of reality (natural, practical, social, and transcendental).

It is in relating to the outside that Ego activates the life of the inner Self, which exists in itself. The inner life has its autonomy, but this autonomy can only be activated and managed in relation to the outside, that is to others and to the social context. For this reason, I call it "vital relationship." It is in this relation that the person can transcend herself, that is tap into a reality that improves life, given that the person cannot transcend herself in a single act or in an external object (animal, plant, machine, or anything else). She does this by activating a reflexive relationality to what is other than herself. It is in this relation that the subject does verification of the uniqueness of

2 P. Donati (2021), 'Relational Essentialism.' In M.S. Archer and A. Maccarini (eds). What's Essential to Being Human? London: Routledge, chapter 4.

3 "Since in the equal (idem) difference disappears, [while] in the same (ipse) difference appears" (Heidegger 1969: 58). 
her identity as human person (as the singular existence of a link between her mind and body) that lives in an environment.

Obviously, the vital relation can generate goods or evils, but what is sure is that the absence or defect of the vital relationship is a problem for human development. The fact that, in a society, outweigh the social evils indicates that people fail to transcend themselves. Without this transcendence, people have an impaired, weak, distorted, fractured identity, and society navigates in the ocean of social evils, such as injustice, violence, corruption, and existential emptiness.

The relational semantics becomes particularly important in the era of information and communication technologies (ICT) because the use of these new technologies creates an inextricable intertwinement between online and offline experience in which the Self is configured as a complex informational system that shows it multiple faces in multiple social contexts through a recursive loop. Hence, the necessity of increasing the reflexive capacities of the relational subject who must find its permanence (the $u b i$ consistam of the idem identity) by continually re-elaborating itself (the ipse identity) in the sea of contingencies. The great challenge of the future lies in the fact that identities change because of the hybridisation of social relations that derives from mixing the logic of the artificial (digital reality) with the natural human logic (analogical reality).

\section{Recognising an identity means creating a relationship}

How does the recognition of identity come about? It happens through relations (per relationem). Paul Ricoeur (2004) investigated the individual act of recognition and concluded that it consists in three moments: (i) the cognitive attribution of a specificity (e.g. if I hear a person speak a strange language that seems German to me, I wonder if she is a German or not), (ii) validation of the attribution (e.g. she is really a German and not a Dutch person who is speaking German), and (iii) gratitude as an appreciation of what exists (in my example, I appreciate that person and I feel sympathetic with her; more generally, this process concerns any existing entity, even non-human).

The relational perspective vindicates the relational character of these operations and affirms that all of this is possible, and, in fact, occurs only in a social network of relations that implement the circulation of mutual gifts. The recognition of identity implies a relationality that is not restricted to the simple one-to-one relationship between the one who acknowledges and the one who is acknowledged.

A practical example can clarify this perspective. Lucia is an Italian volunteer working in a centre for welcoming human life (called CAV, Centro di accoglienza alla vita,) in a big hospital of Milan that specialises in women's health and maternity care. Lucia offers pregnant women who are in trouble because they experience pregnancy as a drama the possibility of developing a free and conscious decision, whatever it is. Lucia observes that they do 
not have a (meaningful) relation either with themselves or with the baby that is developing in their womb. What does Lucia do to help these women? She encourages them to talk, to tell her everything that they can, in a conversation that essentially aims at creating a meaningful relation with her. In this dialogue, the pregnant woman's emotions become Lucia's emotions, and, in their exchange of feelings, reciprocal empathy grows. At this point, their relationship becomes the subject that brings help to the woman (Lucia says that the relation becomes "the subject of care," care being, in other words, taking on the burden of the pregnant woman's suffering). Lucia allows that relation to act reflexively. Living in this relation, the pregnant woman discovers her own identity. In fact, as Lucia says, she is reborn, or, really, "is born" "because she was never born as a person." So, she becomes aware of the choice she wants to make.

In this practical example, we see the causal power of the relation in generating the identity of a human person because she discovers herself through a relational reflexivity. Generally speaking, we discover ourselves, what we are and what we want, (i.e. what most concerns us) from the experience of an "encounter" with an Other that allows us to redefine our identity while being ourselves ("who we are" to ourselves). The relation with the Other, when the Other is committed to creating a We-relation to which the encounter's subjectivity is entrusted, illuminates the totality of the individual human person and gives "form" to her identity because it is capable of penetrating one's deepest being. In brief, the flourishing of human identity is the fruit of the relational reflexivity exercised between people when they are oriented towards building a We-relation from which their individual identity flows.

\section{What is "social" in the relational subject?}

The term "relational subject," as developed by Donati and Archer (2015), indicates individual and collective (social) subjects as "relationally constituted," that is in as much as they acquire qualities and powers through their internal and external social relations. In this chapter, I am interested in seeing what this means for people's identity, that is as regards the role that the relation with the Other plays in defining and redefining one's own identity, whether personal (the identity that the "I" has of itself within itself) or social (the identity that the "I" has as it belongs to a social network with Others).

Obviously, this comes about in different ways depending on whether the subject is individual or collective (such as a family, a sports team, an orchestra, a civil association). In the individual subject, the relation to the Other enters into the individual consciousness and plays a role in the internal self-definition of the individual Self. In the collective subject, the relation to the Other alters the network of relations between the members of the collective subject and, therefore, also the processes that lead to discernment, deliberation, and dedication on the part of the social subject when it acts as a collective. 
I start by asking myself the following question: is the "I" that situates itself in the existing relation with the Other the same "I" that converses internally with itself? The answer is certainly affirmative, because only the "I" reflects, but it is the way in which it "reflects" that is different. Why and how is it different? And what does this diversity engender?

The "I" that converses with itself inside its own mind (inner conversation) has, as an outcome, the fact of rethinking and reprogramming its own deliberations in response to the social context. But one could ask: what outcome is had by the "I" that reflects, not only on itself within itself, and not only on itself in relation to the world, but reflects in/on/with the relation as such with the Other and the world around?

Let us take the example of a musician in an orchestra when she reflects on the quality of her personal performance and when she thinks about and within the performance of the orchestra as a whole and, from this standpoint, evaluates her own performance: are these two ways of reflecting the same thing? This is the problem of the "relational subject." If the musician only thinks about herself, she will seek her personal model of perfection and nothing more (autonomous reflexivity). If she thinks about her contribution as a function of the orchestra, she will seek her best "adaptation" to the orchestra's performance (communicative reflexivity). If, instead, she reflects on/in/with the orchestra's performance and on how this performance can be improved in the event that the musicians relate to each other in a different way, she will seek to alter the performance of the orchestra as a whole, that is she will seek to produce a different emergent effect - which is to say, a more satisfying performance of the orchestra. In this latter case, we can speak of meta-reflexivity. However, it is necessary to distinguish between individual and collective (or relational) meta-reflexivity. The meta-reflexivity of single musicians remains an individual fact that has certain repercussions on the orchestra. It becomes social - "relational," that is related to a collective - when each musician looks (relates) to the orchestra conductor who represents and interprets the We-relation. Therefore, in the conducting of the orchestra, meta-reflexivity appears as the reflexivity of the "collective subject" that is thus because it behaves in a "fully" relational manner.

Sociologists are used to speaking of "collective (or social) subjects" to indicate groups of individuals which act "collectively" in the sense of a collective entity that is supposed to evaluate objectives, deliberate on its own concerns, and strive to achieve them. Examples of collective subjects are a couple, a family, a voluntary association, a cooperative, a labour union, a political party, a foundation, a local community, and a social movement, although, of course, with different relational qualities and causal properties. Both in common parlance and in scientific studies, it is claimed that these collective entities evaluate, deliberate, and "act" as "subjects."

The term "social subject" indicates a collective subject in that it is constituted by relations between individuals that form part of it, and by the external relations that it has in as much as it is expressed in a "We." However, this 
We is not a "thing," it is not an artificial entity, a symbol, an idea, an entity superimposed as a sort of "collective mind," and not even a collective intentionality. We have a "relational social subject" when this We is configured as a relation (We-relation).

What is "social" in the relational subject? The human individual is relational by nature, but relations are created in time and space, that is in a situated sociocultural context. The human person's identity, as reflexive consciousness of the Self, is not a substance in and of itself lacking relations, but is constituted by relations with other human individuals.

In order to understand how the human person is a "relational subject" in a more profound way than the simple Aristotelian assertion that man is a political animal and exists only in society, which is a conclusion drawn from first level empirical observation, we need a sophisticated theory of social relations that is missing from classical ancient thought. Saying that the human individual exists only because he was conceived and raised by other human beings and, moreover, needs other people in order to live, etc., is banal. This says little or nothing about how the human individual is effectively structured (configured) in as much as he is a concrete and situated "relational being." We need second-order observations, as well as second-order (relational) feedbacks.

The problem is to understand how social relationality structures the human individual's personal and social identity as well as agency. When the personal "I" meets the social realm in the form of $M e, W e$, and You, it is necessary to see how it considers these social identities, that is what relation the Self establishes with them. The relations that the Self establishes with $\mathrm{Me}, \mathrm{We}$, and You are very different from one another. An analysis of these dynamics is not within the scope of this chapter, however.

When I claim that a relational subject is a subject that exists only in relation and is constituted by the relations that she cares for, I do not mean to assert that the social relation is a subject in and for itself, but that the relation has its own (sui generis) reality in that it has causal powers and its own qualities. Sui generis (= of its own kind) here means that social relations are the effect or creation of human activities, but they are an emergent phenomenon that corresponds to a different (autonomous and peculiar in its characteristics) order of reality in respect to human behaviour/agency. This relationality (the relation as a real entity) is activity-dependent, but has its own structure, which can be seen in the power that it exercises in retroacting on the terms (Ego and Alter) of the relation itself through relational generative mechanisms (Donati 2015b).

It is not usual that the terms of the relation (Ego and Alter) personally reflect on how the relation between them can be generated or altered by their action. Normally, the relation remains implicit and latent. No wonder the fact that people do not "see" relationships. In the social world, something akin to the physical world happens. We do not see light, we see with light. In the same way, in the social world, we do not see relations, 
but we see with relations, which themselves remain unseen. Most of the time, individual subjects treat the relation with the Other in an implicit and latent manner. They become reflexive subjects in the relational sense ("relationally reflexive") when they succeed in thinking of the relation that they have with other agents/actors as an entity in and of itself and, thus, they consider it as a reality that, while depending on themselves, can in turn "determine" their identity and action. (Here the verb "determine" means that the Ego-Alter relation "circumscribes" the agent/actors with boundaries, "delimits" them, "identifies" them with a certain precision, and "specifies" them).

The underlying question that arises is that of understanding what "the social" is (what we mean by the word "social") when we use it in the expression "social relation." The term "social" is notorious for being extremely ambiguous and riddled with misunderstandings. In general, it is used as a synonym of "collective," in the sense of an aggregation of many individuals. But here, instead, I use it as a synonym of "relational": saying that something or someone is social means saying that it is relational, in the sense that it exists and is relevant for the social fabric (not on other planes of reality) in that it is defined by its social relations. So, the seemingly tautological expression "social relation" refers to the relationality inherent in the relation.

To clarify this point, it is worthwhile remembering that the term "social" is very often used in human and social sciences in the following two ways:

1 as resulting from individual actions, psychological projections or exchanges between individuals who orient themselves reciprocally; here the social is understood as an aggregate effect of a multitude of individuals who produce a collective result; in this case, the orchestra players would not need the orchestra conductor or a soccer team would not need a coach; for example, this is the way in which Uhlaner (2014) explained the onset of the Arab Spring as an aggregate effect of those who participated in the social networks of protest against the political regimes of their countries;

2 or as the structure that overshadows (impinges upon) individuals and makes them think and act in a certain way; in this case, the orchestra players would be no more than the orchestra conductor's executors or the soccer players would be no more than their coach's executors; the social movements of the Arab Spring would then have to be explained by one overarching supernational structure that has caused them, which is highly unlikely.

If one adopts these two meanings of "social," it is not possible to see the relational subject. In the first case, the relational subject is reduced to an "event" or a consequence of individual choices (in a certain sense, a sort of upward conflation is performed); in the second case, the relational subject is 
reduced to a structure lacking agency (in a certain sense, the outcome of a sort of downward conflation).

It is thus necessary to arrive at another meaning of "social," the one that I am proposing here.

3 The social can be understood as "another" order of reality (I call it "the relational order," not to be confused with Goffman's "interaction order"), different from an aggregate reality and from that of a structure overshadowing the agents/actors and which determines them because it consists of relations. It is only in this way that we can explain the emergence of relational goods.

On this point, however, it is necessary to draw some distinctions among those scholars who share the idea that the analytical unit is the social relation, and that it is neither the individual nor structures conceived as a "whole" that determine individuals.

For various reasons, I do not follow those authors who declare themselves to be "relational" but who, in reality, propose a "transactional" sociology because they conceive of the social as "transaction" between individuals (see Emirbayer 1997; Dépelteau and Powell 2013). They should be called transactional sociologies.

The defects of such transactional sociologies are principally these two: in the first place, since they conceive of social relations as the fluid product of situational interactions between individuals, they do not see the sui generis reality of the relations and, in particular, the reality of the social structures constituted by those relations that are not transactional (e.g. the fact of being born of two parents is a relationship that is not transactional); secondly, by reducing the relation to an interaction or multiple interactions, they run the risk of committing a central conflation between agency and structure. On the other hand, I would not call relational sociologies those structural approaches where the social is defined as an outcome of network structural mechanisms, because they ignore the relational feedbacks, which are the engine of the emergence process.

\section{How the relational subject connects personal and collective identity in the morphogenetic process}

Figure 8.1 summarises how structural, agential, and cultural factors affect the formation of the subject's identity in the context of social morphogenesis through personal and relational reflexivity. It focuses on how personal identity and collective identity connect in the relational subject in the course of sociocultural dynamics. This figure is intended as an original elaboration of the SAC (structure-agency-culture) scheme suggested by Archer (2013). The originality of my elaboration lies in the fact that I emphasise the role of relational reflexivity and not only that of the agency's personal reflexivity illustrated by Archer. 


$\begin{array}{ccc}\begin{array}{c}\text { Positions and roles of } \\ \text { the social structure }\end{array} & \begin{array}{c}\text { Subject's } \\ \text { agency }\end{array} & \begin{array}{c}\text { Cultural system that } \\ \text { conditions interactions }\end{array}\end{array}$

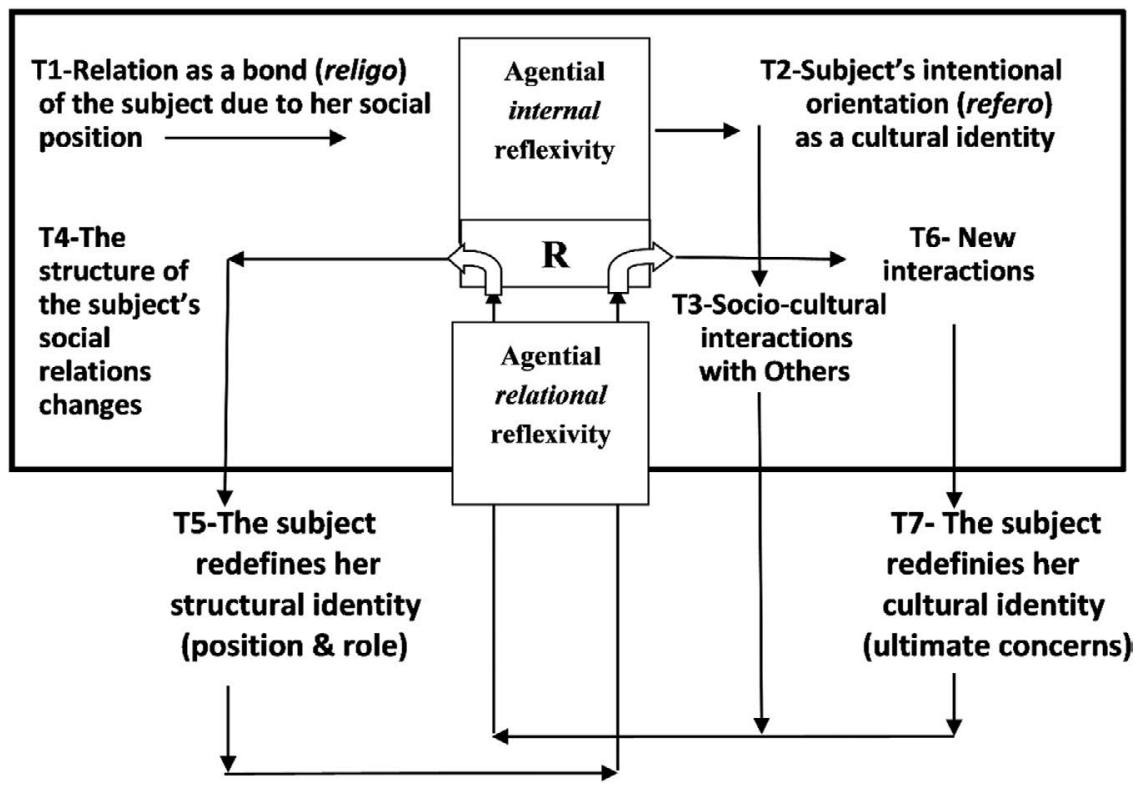

Figure 8.1 The morphogenesis of the subject's relational identity (along the time steps T1-T7)

Source: Author.

The diagram of Figure 8.1 is composed of seven rectangles in a temporal sequence from time $\mathrm{T} 1$ to time $\mathrm{T} 7$, which describe the morphogenesis of the identity of the acting subject that must act within a given social and cultural structure. At time T1, the subject is positioned in a conditioning structure to which s/he responds with individual reflexivity which, at time T2, assumes an orientation in the reference cultural system. At time T3, the interactions with the other actors begin which prompt the activation of the relational reflexivity of the subject who is induced to change his/her relations in the network at time T4. By modifying the relations, at time T5, the subject changes his/her structural identity, that is his/her position in the social structure. This leads the subject to reactivate the relational reflexivity which, at the time T6, modifies the interactions with others and therefore leads the subject to redefine his/her cultural identity at time T7. The diagram outlines a cycle that repeats itself over time, continuing to change the relational identity of the subject.

The acting subject is always already included as an agent in a social structure (in a family, school, professional organisation, voluntary association, social movement, etc.) in which she must define and personalise her role (actor). Initially, therefore, the subject occupies a position/role in a social network on which she must reflect personally (personal reflexivity) having 
to give an answer to the social position that she occupies. In doing so, her inner conversation relates to the context and therefore participates in sociocultural interactions.

In the interactions of the social structure (network) in which the subject is inserted, the social relationship appears as a bond (religo), that is as a link inherent to the roles. In sociocultural interactions, instead, the intentional orientation (the refero) of the subject prevails, made of values, feelings, and expectations that respond to cultural conditioning. The result of sociocultural interactions leads to a redefinition of the Self as a new relational Self. This redefinition of the identity is then subjected to the relational reflexivity of the subject, which translates it into an emerging relationship with the social structure, to redefine her structural identity in the sense of the personalisation of her social role.

The process continues by subjecting again the identity of the structural role played in the social network to relational reflexivity, which is exerted precisely on the qualities and causal properties of the new relationship, which needs to be confirmed in the inner conversation. The symbol $\mathrm{R}$ in Figure 8.1 indicates precisely the connection between personal reflexivity (i.e. personal identity) and social reflexivity (i.e. collective identity), referring to the We-relation. It is this connection that transforms the identity of the relational subject which rests only upon individual interests (the personal concerns understood as what the individual cares about) into a sharing of relational interests (the collective concerns intended as taking care of social bonds and their outcomes) by the subject.

I argue against an integration of agency and structure as it was suggested by some scholars in the debate of past decades. I think it is necessary a shift of focus from solutions relying upon an interplay between agency and structure towards solutions that are based upon the role of social relations, which are a phenomenon on their own, with their own properties and dynamics, which transcend agents and structures. It is the interactive networks between people and structures that are of greatest importance.

On this path, the great novelty of the last years and, in perspective, of our future is given by how ICTs and artificial intelligence (AIs) will influence the hybridisation of social relations in building a relational society according to Figure 8.1. In this regard, I believe that the relational approach is very useful to avoid both the excessive voluntarism of the approaches that emphasise the agential self-reflexivity of the subjects and the positivistic approach of those who emphasise some form of structural determinism, while it is essential to resist to those attempts that support a radical hybridisation of relationships like the Actor-Network-Theory.

\section{Perspectives: human identity vis-à-vis post-/trans-human}

Within the so-called postmodern society, identity - any identity - is called into question to the point that it is proposed that we do without the very 
concept of identity (Brubaker and Cooper 2000) to dissolve it in the pure dynamism of social relations, as if relations did not have their own structure with specific properties and their own causal dynamism.

This pervasive tendency began with so-called identity politics according to which we are "all different, all the same" (this is the slogan of the political ideology of multiculturalism), which has stoked cultural and ethical relativism, has fragmented society and created more conflict than solidarity. In their more general formulation, theories of ideological multiculturalism reject distinctions because they conceive of them as discrimination, and thus they promote a cultural indifferentism that constitutes a fertile ground in which the post-/trans-human can grow. In fact, if the human person does not have a substantial identity, but can be resolved in relations with no residues, then by replacing relations with the spread of new technologies (ICT, AI, robots, etc.), it is possible to create another world, one of androids, humanoids, "electronic persons," developed to replace human persons in order to reach a new stage of evolution (the theory of singularity) beyond human identity.

To the tendencies operating in favour of the transition to the post-/ trans-human, one can and must object that the essence of the human is found in the relation, because from the anthropological point of view, the human originates in the relation, and it is in the relation that persons must learn how to accept their limits, at the same time that they desire to overcome their finiteness, in response to the need for transcendence that characterises the human person in her deepest identity. We will tackle this topic in the next chapter.

Identity is that which generates the relation and, at the same time, is generated by it. Conflations are always possible in every way, but it is up to the "relational rationality" (Donati 2009) to escape the vicious circles. The relational rationality connects the Weberian Zweckrationalität (instrumental rationality) and Wertrationalität (value rationality) in such a way as to give a moral character to social relations.

Persons activate relations based on their own personal identity, but over time, relations change and regenerate their identity. How this happens depends on how relational reflexivity intervenes, if it operates. It is possible to change the relation and, with this, to change identity, just as we can change identity and, as a consequence, change our relations. But it is necessary to see under which conditions this is feasible and with which consequences, in terms of the relational goods and evils that are produced. To avoid falling into tautologies or vicious circles, we must see identity as a "relational subjectivity," that of a substance or nature constituted relationally and evolving relationally.

The major challenges today come from new digital technologies that mediate relationships of all kinds (natural, practical, and social - i.e. within and between individual and collective subjects). This topic will be addressed in the next chapter. 


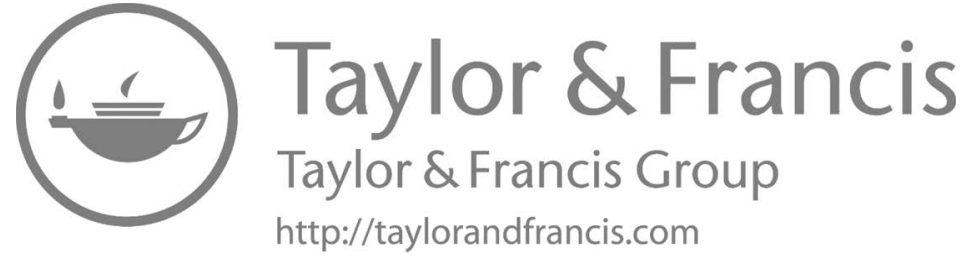




\section{Part IV}

The hybridisation of society: shall we forget about its human character? 


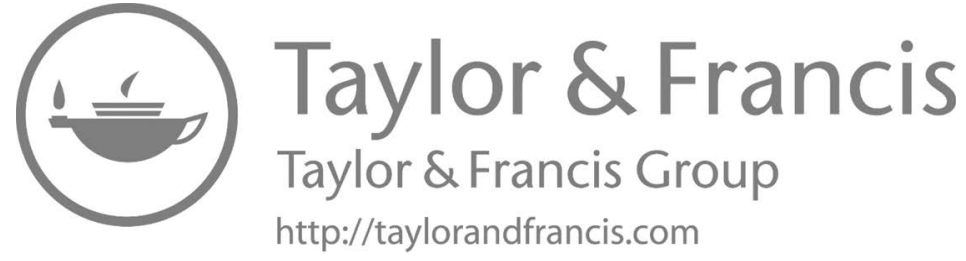




\section{The new scenario of a hybridised society}

\section{The topic and its rationale}

In this chapter, I propose an analysis of how the processes of human enhancement brought about by the digital revolution (information and communication technologies [ICT], artificial intelligence [AI], robotics) are modifying social identities, relationships, and social organisations, and under what conditions this revolution can shape organisational forms that are able to promote, rather than alienate, humanity.

A recent debate in France about the use of robots in care for the elderly ended with the question: "can the human relationship be the product of our technological feats?." We must look for a plausible answer.

Personal and social identities, relations, and organisations are forced to take shape in the environment of a Digital Technological Matrix (henceforth DTM) that is a symbolic code, which tends to replace all the ontological, ideal, and moral symbolic matrices that have structured societies in the past. The concept of matrix used here refers to the meaning that this concept has in my book on the theological matrix of society (Donati 2010). It indicates a vision of the world that has a religious character, or functionally equivalent to that of religion, in that it expresses the "ultimate values" of that society. In the case of DTM, these values are those of an indefinite evolution of humanity and the cosmos that replaces the representations of the world as a reality created and supported by a supernatural reality. ${ }^{1}$

Obviously, I am talking about a trend, not only because in each society different cultural matrices coexist, in particular in the multicultural societies emerging today, but also because it is always possible that the conflicts between cultural matrices can intensify and diversify. What I argue is that DTM, like all cultural matrices that have a transcendental character, that is express a vision of the cosmos and humanity in their ultimate ends (éschata, the ends of everything), has an intrinsic propensity to become the dominant matrix, similarly to what happened with the cultural matrix of modernity

1 The values I am referring to are well illuminated by Harari (2017) and by various contributions to the volume of Floridi (2015). 
that made traditional cultures marginal. As a form of Tech-Gnosis, the peculiarity of the DTM is that of making the boundaries between human and non-human labile and crossable in every way in order to foster hybrids. Hybridisation is defined here as entanglements and interchanges between digital machines, their ways of operating, and human elements in social practices.

Hybrids, however, are not random or purely contingent entities. They stem from complex interactional networks, in which social relations are mediated by the DTM. The processes of hybridisation of social identities and relations are selective and stratified according to the ways in which the human/non-human distinction is relationally thought of and practiced. We are witnessing a co-evolution between $\mathrm{AI} /$ robotisation and human relations.

Three scenarios of hybridisation are outlined along with three kinds of societal morphogenesis: adaptive, turbulent, and relationally steered. The chapter examines these scenarios of co-evolution and their possible consequences.

I am not so interested in discussing whether AI or robots can be more or less human in themselves - or not human at all - but rather how they can interact with humans and affect their social relationships so as to generate a different kind of society characterised by the hybridisation between human and non-human.

I will tackle two major themes: (i) the first concerns the problem of how we can distinguish inter-human relations from the relationships between human beings and machines, which implies the need to clarify what the processes of hybridisation of identities and social relations consist of and how they happen; (ii) the second theme concerns the consequences of digitalised technological innovations on the hybridisation of social institutions and organisations and, ultimately, the possible scenarios of a hybridised society.

\section{The hybridisation issue}

The hybridisation of society emerges from a process of social morphogenesis in which social structure, culture, and agency intertwine so to produce social and cultural hybrids together with a hybridised agency. Figure 9.1 schematises these processes, which consist in a series of subsequent morphogenetic cycles.

In principle, each morphogenetic cycle can start from the side of the social structural domain or from the side of the cultural domain. Let us see what happens in either case.

1 If the morphogenetic cycle begins on the side of the structural domain, for example when a robot is introduced in a certain social context (a factory, a school class, etc.), what happens is a redefinition of the social network and the social positions of the actors involved. As a consequence 
of the presence of the newcomer, structural interactions change and produce structural hybrids. These hybrids affect human agency in the direction of influencing their impact on the cultural domain.

2 If the morphogenetic cycle starts from the side of the cultural domain, for example when new software (digital literacy, programs, algorithms) are invented and adopted by people, then new sociocultural interactions produce cultural hybrids. These cultural elaborations modify human agency, which introduces changes into the structural domain.

3 The human agency changes through active (reflexive) responses to the above changes. The possibilities for developing this morphogenetic process are certainly dependent on how the DTM operates in the cultural and structural domains, but we must consider the autonomous role of human agency.

One may wonder, which morphogenesis occurs in the human person (agency) and in his/her relational qualities and properties? What happens to social relations between humans and between humans and robots?

Figure 9.1 illustrates the relational structure and dynamics of co-evolution between digital machines, their ways of operating (according to their symbolic code), and human agency illustrates this process in case it starts from the social structure. Co-evolution can obviously have positive or negative results in terms of compatibility among the different components, and therefore, it can generate both relational goods and relational evils.

The diagram in Figure 9.1 is composed of eight steps in a temporal sequence from time $\mathrm{T} 1$ to time $\mathrm{T} 8$ which describes the hybridisation of the acting subject and his/her relationships when operating with new digital technologies, for example when an AI or robot is introduced into the subject's relational network. At time T1, the introduction of the new technology (hardware) modifies the structural network of the subject's relationships, and thus modifies his/her position or social role. In the T2-T3 time phase, interactions in the network activate hybridisation processes of the social structure, so that structural hybrids emerge at time T4. The structural hybrids lead to hybridise the action of the subject, who is depicted in a central rectangle. The next step, at time T5, indicates that, following changes in the social structure, the subject changes his/her cultural identity in reference to digital software. This step is followed by a phase of interactions between time $\mathrm{T} 6$ and time $\mathrm{T} 7$ in which sociocultural interactions activate cultural hybridisation processes. Consequently, at the time T8, cultural hybrids emerge that condition the subject's action. As in previous figures, it is a typical cycle that repeats itself over time.

Notice that the morphogenesis of human agency is both passive and active in both directions, towards the cultural as well as the structural domain. (i) It is passive because it is influenced alternatively by one of the two domains, and (ii) at the same time, it is active towards the other 


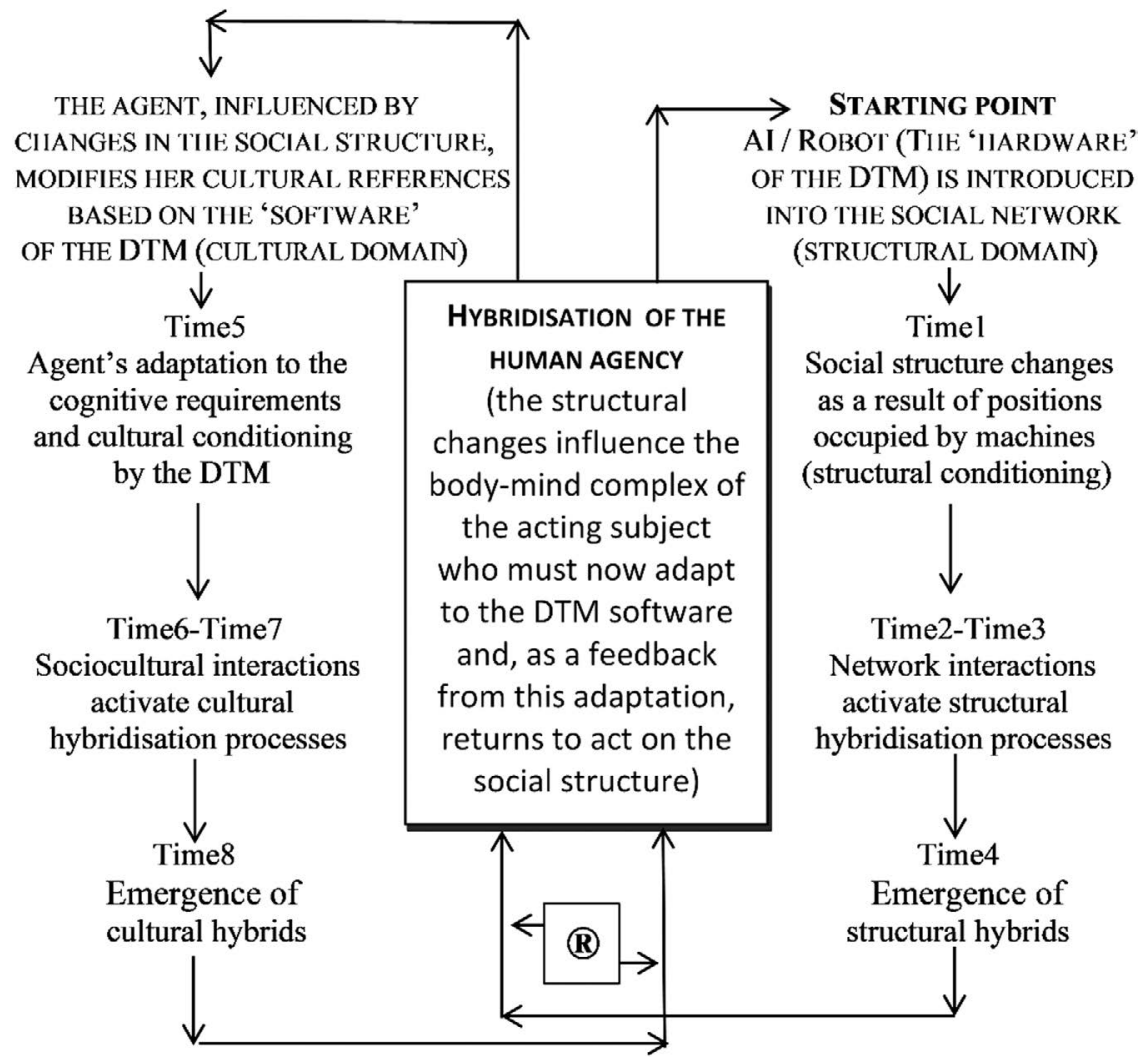

Figure 9.1 A scheme of the process of hybridisation (structural, agential, and cultural)

Source: Author.

domain. It is here that the body-mind relational unit has to confront the coherences or dissonances between the two domains. The symbol ${ }^{\circledR}$ means that there is a connection of some sort between the cultural and structural processes of hybridisation of the human person in his/her social identity and social relationships. Such a connection can be of different kinds, from the maximum of synergy (complementarity), as when cultural identity is well adapted to the position occupied in the social structure, to a maximum of conflict (contradiction), as when cultural identity conflicts with the position occupied in the social structure.

What is most relevant in Figure 9.1 is to observe the dematerialisation of the human agency due to AIs operating in and through the quantum network (the Internet) where information is transmitted with qubits. The process of hybridisation takes place in fact contaminating the relationships that operate on the basis of the principles of classical physics applied to the 
natural world with virtual relations that operate on the basis of the postulates of quantum physics, where the latter dematerialises the natural world. ${ }^{2}$

The conceptual scheme summarised in Figure 9.1 is fundamental to understand the hybridisation processes of social identities, relationships, and organisations in the light of the idea that, to speak the language of Adams (2006: 517), "hybridity becomes a complex potential." These processes proceed according to a relation of contingent complementarity between changes in the cultural background that encourage the development of certain forms of virtual thinking and utopian discourse, on the one hand, and changes in the material production of new technologies (AI/ robots) in the structural domain, on the other hand. Hybridisation occurs when culture (software) and structure (hardware) mutually influence and reinforce each other, as long as human agency adapts to these processes. Relations play the most crucial role because changes in identities and organisations depend on them. The hybridisation of society is based on the assumption that these processes operate in a functional way, making sure that the passivity of human agency translates into its active participation in such an evolution with minimal reflexivity or a reflexivity substantially dependent on the DTM. The kind and extent of hybridisation depend on how subjects reflect on their relationships, on which their internal conversation (personal reflexivity) depends.

The hybridisation of people's identities and social organisations (families included) consists in the fact that these entities change their relational constitution to the extent that the cultural and structural processes of change affect (in Figure 9.1 they "cross") the human person and modify his/her ways of relating to himself/herself, to others, and to the world. Agency is obviously dependent on how the brain and the mind of the person work. Both the brain and the mind are seriously influenced by the way technologies operate (Greenfield 2014) because they absorb certain ways of communicating, using and combining information, and following performance logics that can ignore or alter analogical thinking. As Malabou (2009) claims, the human being has his essential difference in the plasticity of his mind that the robot will never have (synaptic plasticity is unique, there cannot be two identical brains).

Since hybridisation means some form of mutual adaptation between humans and AI bots, one can ask to what extent this mutual influence (up to mutual constitution) can arrive. Personally, I do not believe that humanoid robots can be or become "relational subjects" in the sense of being able to relate to others and to the social context by forming a We-relation with

2 For a debate on this issue, see the theory by Alexander Wendt (2015) according to which consciousness is a macroscopic quantum mechanical phenomenon and the critical review by Porpora (2018b), in particular for what concerns the consequences of Wendt's ideas on social life. 
adequate personal and relational reflexivity. They cannot for various reasons, in particular because their mind and their body-mind complex are ontologically different from that of humans, so that human-human interaction (HHI) and human-robot interactions (HRI) are ontologically different (of course, if we assume that human relations are constituted by reciprocal actions which are something more and different in respect to pure behaviour). Basically, AI bot's mind (consciousness) has an inside-out and outside-in relationality that is structurally different from the human one (Lindhard 2019). There is much debate about whether robots can be friends of human beings. My position is that it is unlikely that humans will ever be able to be friends with robots. The capacity to enter into a friendship of one's own volition is a core requirement for a relationship to be termed friendship. We also have a duty to act morally towards our friends, to treat them with due respect. To be able to do this, we need to have self-knowledge, a sense of ourselves as persons in our own right. We do not have robots who display these capacities today, nor is it a given that we ever will.

Bearing in mind the substantial differences between social relations and technological relations (Nørskov 2015), I believe however that it is possible to recognise that "social robots, unlike other technological artefacts, are capable of establishing with their human users quasi-social relationships as pseudo-persons" (Cappuccio et al. 2019: 129).

Hybridisation means that, through sustained interactions with technologies (the "fully networked life"), the previous modes of considering (conceiving) oneself, relationships with others, and what form to give to a social organisation in keeping with the (analogic) principle of reality are mixed with the way digital technology works, that is the (fictitious) principle of digital virtuality. Hybridisation means blending the real and the fictitious, the analogical and the digital. This happens, for example when one wants to modify one's own image through an app that presents it in an altered form in shapes and colours in a social network.

To clarify this point, I must say that I do not use the term "real" in opposition to digital. In my view, the polar opposites are "real" vs "fictitious" and "digital" vs "analogical." If a hacker creates a profile on Facebook, or another social network, and transmits fake news, such news has its own reality, even if it is the reality of a fiction. While the story's referent is not real, the story itself is causally efficacious and therefore real in that regard. So, instead of contrasting a digital message to a real message, it is better to contrast a digital message with an analogical, because the analogical code implies, in fact, an effective analogy relationship between what is written or transmitted and what is really existing. In short, hybridisation can take place by putting in interaction and then mixing what is real and what is fictitious, what is digital, and what is analogical. Table 9.1 presents four types of relationships that generate hybrids when they are conflated with each other.

Qualitative and quantitative research provides some empirical evidence of these hybridisation processes: 
Table 9.1 Four types of relations generating hybrids when mixed together

\begin{tabular}{|c|c|c|}
\hline & Real & Fictitious \\
\hline Analogical & Face-to-face relationships & Plays on relationships \\
\hline Digital & $\begin{array}{l}\text { Digital relations that } \\
\text { replace face-to-face ones }\end{array}$ & Virtual simulation relationships \\
\hline
\end{tabular}

Source: Author.

- About ICTs: in the chapter "Twenty-First Century Thinking," Greenfield (2009) suggests that the decline of reading in favour of fragmentary interactions, such a computer games or short messages on the Internet, threatens the substance of both our neurological makeup and our social structures.

- human persons alter their pace, rhythm, and sense of Self; they change their personal reflexivity.

- About AI/robots: authentic human relationships are reduced to and sacrificed in favour of digital relationships (we expect more from technology and less from each other); technology appeals to us most where we are most vulnerable (passions, feelings, interests, etc.).

- Digital relations erase precisely those aspects of randomness that also make human life, people, and relationships interesting, spontaneous, and metamorphic.

- By developing new technologies, we are inevitably changing the most fundamental of human principles: our conception of self, our relationships to others, and our understanding and practice of love and death; nevertheless, we should not stop developing new technologies, but do it differently by adopting an approach of relational observation, diagnosis, and guidance.

In short, the processes of hybridisation of identities, human relations, and social organisations are closely connected and change together (in parallel with each other), varying from case to case due to the presence of intervening variables in specific contexts.

\section{The process of hybridisation of identities and relations}

From the point of view of critical realism, hybridisation can and must instead be considered as a morphogenetic process that leads from entities structured in a certain way to entities structured in another way. The fact remains that we should understand how to define the proprium of the human in hybridisation processes.

Lynne Rudder Baker's theory is often cited as a solution to this problem as it replaces a "constitutive" rather than a substantive (so-called identifying) view of the human. 
Baker (2000) argues that what distinguishes persons from all other beings is the mind's intentionality detached from corporeity since we, as human beings, can be fully material beings without being identical to our bodies. In her view, personhood lies in the complex mental property of first-person perspective that enables one to conceive of one's body and mental states as one's own. The argument is that the human mind must have a bodily support since the body-mind relation is necessary, but the type of body - and, therefore, the type of body-mind relationship - can be quite contingent; according to her, we can change the body with artefacts, provided we do so from the perspective of the first person. The relation between one's mind and one's body is open to any possibility. Consequently, since the personality is equal to the self-thinking mind, we must then acknowledge the existence of a personality in any animal or machine that can be judged sentient and thinking, on the condition that it is aware that it is itself that thinks. In this way, the possibility of anthropomorphising robots, as well as robotising human bodies, becomes thinkable and legitimised.

Following this line of thought, some thinkers today are more and more inclined to acknowledge the existence of moral behaviours in certain species of higher primates like the chimpanzees. They are supposed to have a "moral intelligence," that is in that they are compassionate, empathetic, altruistic, and fair (Bekoff and Pierce 2009), and, along the same lines, they recognise the possibility that special robots endowed with an artificial morality might exist (Pana 2006). It seems to me that this perspective is totally at odds with a realistic social ontology for which human relationality - between body and mind, as well as in social life with others (the two are closely related) - has qualities and properties that cannot be assimilated with the relationships that certain species of animals or ultra-sophisticated intelligent machines can have. On the whole, Baker's theory is inadequate to the task of accounting for the possibilities and the limits of the processes of hybridisation because it does not account for the relational constitution of identities and social forms.

The hybridisation of human relations, paradoxically, is due to the logic inherent in such relationships, which, by definition, must be open to the world and cannot remain closed in self-referentiality, as artificial machines do. In interacting repeatedly with machines (AI/robots), agents can incorporate certain aspects of the communication logic of the machines in their way of relating to others and the world. However, we cannot equate the relations between humans with those between humans and robots. How can one not see that the qualitative difference between these relations, for example when someone says that the robot is "my best friend," reduces the relationship to pure communication (i.e. to communication and only to communication, as Luhmann 1995 states)? This is an unjustified and unjustifiable reduction because communication always takes place within a concrete relationship and takes its meaning, qualities, and properties from the kind of relationship in which it occurs, whereas the kind of relationship depends on the kind of subjects that are in communication. 
The problems caused by DTM (ICTs and robotics) arise when sociability is entrusted to algorithms. The introduction of the sentient robot changes the context and the relationships between human subjects as well as the form of a social organisation.

Let us take the example of a social structure of a sports type like that of a football team and a football match. How does the behaviour of a football team that is equipped with a goalkeeper-robot capable of parrying all rolls change and if two teams agree to play a game in which each of them has a robot as a goalkeeper, what will be the consequences on human behaviour?

There cannot be a "we believe" or a relational good between humans and robots for the simple fact that the supervenience human-robot relation is ontologically different from the supervenience relationship between human beings. ${ }^{3}$

The partial or total hybridisation cycle of relationships is selective and stratified on the basis of (i) how the personal and relational reflexivity of agents operates, given the fact that reflexivity on these processes is necessary to get to know each other through one's own "relational self-construal" (Cross and Morris 2003) and (ii) how the reflectivity of the network or organisational system in which the agents are inserted operates.

We can see this by using the morphogenetic scheme, which describes how the hybridisation processes depend both on the reflexivity of the subjects and the reflectivity of their social network exert on the different forms of human enhancement.

At time T1, we observe an organisational context (or network) in which human persons must relate themselves to a DTM of sentient machines. In the temporal phase T2-T3, persons and sentient machines interact with each other. The hybridisation of social relationships and identities can happen in various ways, and with different intensity, depending on whether the human person adopts:

a A conformist reflexivity, that is a reflexivity that is purely dependent on the machine; in practice, the agent person relates to the machine by identifying herself with the digital code, which means that she "connects" to the machine without establishing a real relationship (connection is not a social relationship); it happens, for instance, when people identify themselves with their Facebook profile.

3 A set of properties $A$ supervenes upon another set $B$ just in case no two things can differ with respect to $A$-properties without also differing with respect to their $B$-properties. In slogan form, "there cannot be an $A$-difference without a $B$-difference" (Stanford Encyclopedia of Philosophy 2018). In our case, the human-robot relationship would be equivalent to the human-human relationship if the robot were human. 
b An independent reflexivity, that is a reflexivity that is autonomous with respect to the machine; the machine is used as a simple tool, and the human-robot relationship follows basically an analogic code.

c A critical reflexivity (meta-reflexivity) which means that the agent discerns the meaning of relations on the basis of the difference between the digital and the analogical code by re-entering 4 the distinction digital/ analogical into what has emerged in previous actions and their outcomes; if the agent uses the interaction s/he has with the machine in this way s/he can redefine the purpose of interaction, in order to establish a more satisfying relationship.

d An impaired reflexivity, that is an impeded reflexivity that is caused by an absorbing routine identification of the agent with the machine.

e A reflexivity that is fractured due to the fact that the agent combines the digital code and the analogical code in a casual and fuzzy way.

At time T4, a new social network emerges which exerts its reflectivity on agents and machines. At this point, a new morphogenetic cycle starts.

As Lally explains, "The development of the human subject (individual and collective) takes place through a progressive series of processes of externalisation (or self-alienation) and sublation (reabsorption or reincorporation). Human subjects and the (material and immaterial) objects of their sociocultural environment form a subject-object relation which is mutually evolving, and through which they form a recursively defined, irreducible entity" (Lally 2002: 32). These subject-object sociotechnical associations constitute the material culture of everyday life; they are bundled together with the affective flows of human relations and the emergence of a life-world in which images and emotions play a crucial role in the hybridisation of relationships, because it is through them that the subjects identify with the logic of virtual-digital relationships (LaGrandeur 2015).

The hybridisations of social relations and human identity through the DTM are connected to each other because the human being is a relational subject. The dynamics of mutual influence between identity and relations in the hybridisation processes are not linear, but proceed along very different paths between the human person as a natural being (body, mind, and moral conscience), the human being a practical user of technologies (ICTs, AIs, robots), and the human being as a social subject within the networks of social relations. Let us see the hybridisation of human identity through these different paths of interactions.

4 I am referring to the re-entry operation proposed by Spencer-Brown (1979) and subsequently used by Luhmann. I have taken it up by interpreting it in a relational key to define the human: see P. Donati. 'A Critical Realist Perspective on Humanness as a Meaningful Re-Entry of Relational Distinctions.' Journal for the Theory of Social Behaviour, first published: 15 October 2020 https://doi.org/10.1111/jtsb.12252. 
1 There are two-way interactions between the natural being and the use of technology (regardless of the social context). From a phenomenological point of view, the relational context is always there, but in many cases, the human agents do not take this into account, as often happens in medical settings and biological laboratories.

Examples of this type of hybridisations are those widely used in medicine, where they involve checking whether the technology helps repair damaged organs or cure diseases. Baker (2013) generally refers to this type, obviously considering the individual as natural beings. But even when she speaks of human enhancement in the proper sense, that is as making someone or something "more than normal" by applying digital technological devices that are well beyond therapy, she does not consider the implications on the level of the social context. Since the relational order (context) is practically ignored in her theory, Baker's vision remains restricted to a mentalised interpretation of the hybridisation of the individual.

2 There are two-way interactions between the use of technology and sociality. In this case, the human agent is a virtual person who disregards his/her body-mind constitution. An example of this type of hybridisation is the phrase: "I do not use the blog; I am the blog." Hybridisation happens through the formation of a relationship in which Ego's identity is its blog. There is an identification of subject and object: no longer, "I make my blog in order to communicate," but, "I am my communication" (Stichweh 2000). The logic of the blog becomes part of the logic of the Subject, who reflects according to the logic of what was once an instrumental object (a means) and now has become his/her way of relating (a norm), which reflects a value placed in the communication itself, in the presence of an undetermined goal.

The dialogue between the " $I$ " (i.e. the Self that dialogues with itself), its "Me" (i.e. what the "I" has done in the past), and its "You" (i.e. what the "I" is willing to do in its future) $)^{5}$ takes place within an interactive time register, in which time is evenemential, that is has no durée (it is composed of a sequence of events each without duration). It is neither relational (the time of relationships that have a beginning, a duration, and an end), nor symbolic (the time of symbols that have no time, those that do not depend on time), but is purely interactive (appears and disappears with every single communication). ${ }^{6}$ The temporal history of the body-mind reflexivity, its stock of knowledge (Elster 2017), does not enter the process, except for the

5 This sequence is elucidated by Archer (2013, Figure 2, p. 4).

6 On the three registers of time interactional, relational, and symbolic, see Donati (2011a: 180-181). 
aspects in which it has been mentalised. The identification of the human person with the blog is the outcome of the process of mentalisation, which corresponds to the fact that an AI has become part of the Subject's Mind, forging its conscience.

3 There are two-way interactions between the agent as natural being and sociality. In this case, the individual acts regardless of the technology, and, therefore, there is no hybridisation. This is the case, for example of families of elderly people who do not use digital technologies or of voluntary organisations that take care of the poor and fragile without resorting to any technological tool.

4 There are processes in which the agents are involved in all three orders of reality. Their body-mind complex and their sociality are mediated by technology, which is what happens "normally," in fact, in any institution and organisation. The kind and degree of hybridisation depends on the network structure in which the agency is embedded.

It is important to emphasise that hybridisation of social identities and organisations through the use of technology happens by involving the mind-body complex in the context of relationships, without the mind being separated from the body. The mind of agents/actors is modified through the perception and activation of those social relationships that are possible only through the body.

Victoria Pitts-Taylor offers an account of how the mind works on the basis of a brain that is "complex and multiple, rather than determined and determining" (Pitts-Taylor 2016: 8). Drawing on work by feminists, queer theorists, and disability theorists, she offers an understanding of bodies and cognition that can incorporate the cognitive differences that result from differences in embodiment and that can recognise the social shaping of both bodies and cognition. She notes that scientists have discovered that some neurons seem to respond both to our performance of an action and to our observation of others performing that action. These mirror neurons have been theorised to underlie social relationships, especially "mind reading" and empathy. We need to do a better job of recognising the complexity and variety of social relationships. In particular, the assumption that the existence of mirror neurons shows that we are naturally in tune with others and empathetic to them leaves social neuroscientists unable to account for why our empathy for others is so often selective and so often fails. This is an example of how social neuroscience can take the embodiment of brain and social relationship seriously, differently from those attempts to create digital identities as avatars that simulate the human without being human (e.g. the Soul Machines company), thus producing a morphic sociability devoid of real humanity (Pitts-Taylor 2016).

Let us now move from the micro to the meso and macro levels of the social fabric, to consider the institutional and organisational hybridisation processes. 


\section{The emergence of hybridised institutions and organisations}

I call hybridised organisations and institutions those that pursue a social configuration in which advanced digital technologies are conferred a certain degree $(\mathrm{min} / \mathrm{max})$ of decisional and operational autonomy. Technology (AI/robotics) takes on an autonomous and decisive role in managing the organisation of roles and relations between the members of the organisation. The digital logic with which hybrid organisations operate is that of increasing opportunities, conceived, however, not within the framework of their relational implications, but according to the maximum useful variability in terms of system efficiency.

The relational approach to social organisations can show why and how $\mathrm{AI}$ and robots cannot replace humans because of the specific generative character of inter-human relations. In fact, the utilitarianism of efficiency refers to the relationships between an actor/agent and "things," while the search for relational goods - or the avoidance of relational evils - implies relationships between human beings, which, unlike algorithms, are generative of meta-reflexive solutions for problems of human relationships. ${ }^{7}$

Let me give an example of how an algorithm can generate relational evils. On November 21, 2017, the algorithm of the multinational company Ikea fires a worker of its megastore in a small town near Milan. Marica, a 39-year-old mother separated from her husband with two young children, one of whom is disabled, is fired because she does not observe the new work shift assigned to her by the algorithm. The algorithm has ordered her to show up at 7 a.m., and, instead, she arrives at 9 o'clock according to the old work shift because she has to look after the children, and, in particular, she has to take the disabled child to therapy. Previously, the woman had explained to the manager that she could not work that shift, and the manager said that he would consider her situation, but the algorithm worked on its own and fired her. The company did not review its decision, and, instead, continued to dismiss other workers on the grounds that they did not comply with the indications of the algorithm.

Undoubtedly, it cannot be said that the algorithm has not been proven to have its own "personal" decision-making ability (some call it "electronic person"), but it was certainly not a relational subject. Clearly, the algorithm

7 By relying on the notion of auctor proposed by Donati, Scott Eacott (2018: 85) observes that "Auctor provides the intellectual resource to overcome the structure/agency analytical dualism through recognition of relations and the generative rather than deterministic nature of activity without defaulting to a naïve form of autonomy. [...] Auctors generate organising activity. They are not separate from spatio-temporal conditions but simultaneously constitutive of and emergent from them. The substantialist basis of organisational theory in educational administration and leadership is broken down through relations. This is why, despite my (somewhat artificial) partitioning of organising activity, auctor, and spatio-temporal conditions here, they work together, in relation, to generate an elaborated communication of activity." 
simply followed its procedural rules, neglecting the needs of people and their relational context, which were unpredictable for him. If so, we can say that the algorithm is not a person, given the fact that a person is an "individual-in-relation" (relationality being constitutive of the individual), although it is presented as such by management. The algorithm's personality is a convenient managerial fiction.

In the perspective of the future, in my opinion, in principle, it will be possible to build more sophisticated $\mathrm{AI} /$ robots that can take into account people's needs and their relationships. However, in addition to modifying the instructions provided to the algorithm accordingly, it will be always necessary to supervise its operations by a management that, by adopting a relational steering approach, must be able to deal with the individual problematic cases and the complexity and diversity of contingencies that the algorithm cannot handle. Without such relational steering, the hybridisation of organisational relationships due to increasing automation will only mean exploiting people and dismissing them by blaming the algorithm for making inhuman decisions and apologising for not being able to do otherwise.

From a more general view, as Teubner writes, "Today, with the massive emergence of virtual enterprises, strategic networks, organisational hybrids, outsourcing and other forms of vertical disaggregation, franchising and just-in-time arrangements, intranets and extranets, the distinction of hierarchies and markets is apparently breaking down. The boundaries of formal organisations are blurring. This holds true for the boundaries of administration (hierarchies), of finance (assets and self-financing), of integration (organisational goals and shared norms and values) and of social relations (members and outside partners). In formal organisations, membership becomes ambiguous, geographical boundaries do not matter much anymore, hierarchies are flattened, functional differentiation and product lines are dissolved" (Teubner 2002: 311).

Hybrids raise problems of conflict between divergent normative arrangements. As a strategy to deal with these changes, Teubner recommends a "polycontexturality which combines heterarchy with an overarching unity" (Teubner 2002: 331) assuming that this organisational solution would represent the new institutional logic capable of avoiding collisions between spheres ruled by incompatible norms.

I take Teubner's proposal as an example of an attempt to preserve the human (with its regulatory requirements) alongside the acceptance of the entry of new hybrids, through general rules that allow different norms to coexist in different domains (Teubner 2006b). Unitas multiplex is his key word for preserving the integration of society, supposing that human beings and actants, human relations and artificial relations, could coexist within a neo-functional differentiation architecture under the aegis of the DTM.

I have serious doubts about the tenability of this perspective (although it worked in the 17th century as the Hobbesian solution to the problem of social order). I think that it is an impracticable solution in a networked 
society governed by the DTM. In any case, it does not work for a "society of the human." As Hobbesian (Leibnizian) rationalism, Teubner's idea of a constitutionalisation of the private hybridised spheres does not address the issues of the relations between human and non-human, and the relations between divergent normative spheres. Teubner's perspective simply avoids the relational issue. In short, it has all the limitations of a multicultural doctrine that ignores the reality of what lies in between opposite spheres that have incompatible normative orders. It is silent about the issue of how far the hybridisation processes can go and to what extent they can affect the human.

The same difficulty is present in the Luhmannian theory that seems to do a good job of interpreting the hybridisation processes insofar as it places all cultures under the aegis of a conception of society as an "operating system" (Clam 2000). According to Luhmann (1995), all systems, organisations, and interactions are forced to use a binary functional code that is precisely the one through which the DTM proceeds. Hybridisation follows a functional code. For this reason, he believes that the humanism of the old Europe is unsustainable and, therefore, sees no other way than that of considering the human (especially the human of the Western tradition) as a residual entity fluctuating in the environment of the DTM. Are there no alternatives? I think that there are. We must examine the possible scenarios.

\section{Three scenarios dealing with the processes of hybridisation}

The digital transformation of society is destined to produce different types of hybrids through different types of social morphogenesis. I would like to summarise them in three scenarios: adaptive morphogenesis, turbulent morphogenesis, and relationally steered morphogenesis.

1 Adaptive $M G$ producing hybrids by trial and error: this is the scenario of a society that adapts itself to the hybridisation processes produced by DTM in an opportunistic way; it is configured as an afinalistic infosphere - without pre-established goals, as in Floridi (2015) - that tries to use the technologies knowing that they have an ambivalent character: they allow new opportunities but also involve new constraints and possible pathologies; therefore, it is essentially engaged in developing self-control tools to limit emerging harms (theories of harm reduction).

2 Turbulent MG favouring mutations: this is the scenario of a society that operates for the development of any form of hybridisation; it becomes "normal" to anthropomorphise the robots, as well as to robotise the human body: in principal, it is run by anormativity and anomie (lack of presupposed moral norms) and openness to mutations, understood as positive factors of "progress" (theory of singularity); it operates through ceaseless, unrepeatable, intermingling processes of relational flows with "confluence of relating" (Shotter 2012). 
3 Relationally steered MG aiming to configure technologies in order to favour relational goods: this is the scenario of a society that tries to guide the interactions between human subjects and technologies by distinguishing between humanising and non-humanising forms of hybridisation. The aim is to produce social forms in which the technologies are used reflexively in order to serve the creation of relational goods. This requires that producers and consumers of technologies work together interactively, that is that they are co-producers and partners in the design and use of technologies, careful to ensure that technologies do not completely absorb or replace human social relations, but enrich them. This alternative is certainly much harder to pursue than harm reduction, but it is not impossible, and it is the one that leads to good life. ${ }^{8}$

Hybridisation cancels every dualism. In particular, it erases the dualism between the system and the lifeworld (theorised by Habermas 1987) and replaces it with a complex relational system in which each action/communication must choose between different causal mechanisms (Elder-Vass 2018).

To be human, technological enhancement must be able not only to distinguish between the different causal mechanisms, but also to choose the most productive social relationships that can generate relational goods. New technologies generate not only unemployment, as many claim. They also release energy for the development of many jobs in the field of virtual reality and make it possible to put human work into those activities that have a high content of care, such as education, social assistance and health, or a high content of cultural creativity.

Laaser and Bolton (2017) have shown that the introduction of new technologies associated with the advance of performance management practices has eroded the ethics of the care approach in banking organisations. Under electronic performance management monitoring in bank branches, in particular, co-worker relationships have become increasingly objectified, resulting in disconnected and conflict-ridden forms of engagement. This research reveals the multilayered and necessarily complex nature of co-worker relationships in a changing, technologically driven work environment and highlights the necessity for people to defend the capacity to care for others from the erosive tendencies of individualised processes. Within the relational approach, this entails assessing the way in which an organisation uses $\mathrm{AI} /$ robots to enhance human relations from the viewpoint of what I call "ODG systems" (already illustrated in Chapter 2) aimed at the relational steering of digitised organisations.

ODG systems, based on the sequence: relational observation $(\mathrm{O}) \rightarrow$ relational diagnosis $(\mathrm{D}) \rightarrow$ relational guide $(\mathrm{G})$, can make use of $\mathrm{AI} /$ robots

8 Practical examples can be found in the journal "Relational Social Work" (free access). 
as means for communication, information sharing, and decision-making. The cooperative norm is obviously inserted as a basic standard in the AI/ robot that supports the agents of the social network. The sequence works like that. (O) Relational observation aims to define the problem to which AI must respond in terms of a problem that depends on a certain relational context. Therefore, it favours the meso level (in which relational goods can be produced). (D) Relational diagnosis aims to define the satisfactory (or not satisfactory) conditions with respect to the effects produced by the way the AI works on the relational context (i.e. whether the AI contributes to produce relational goods instead of relational evils). (G) Relational guidance aims to modify the AI and its way of working to support a relational context that can be mastered by people in order to generate relational goods.

An example of product innovation can be that of systems that try to regulate the AIs of self-driving cars. The AI must be constructed in such a way as to take into account the main parameters of the relational context in which it operates. The AI must see objects and people around, and assess their relationships with respect to those who sit in the driver's seat to put him in a position to intervene on situations that present a high contingency. Relational observation implies the ability of AI to calculate the relationships given in a context and those possible in its shortmedium-range evolution. Relational diagnosis concerns the ability to perceive possible clashes in case the relationships with objects and people can become dangerous while the car is on the street. Relational guidance means the ability to regulate these relationships in order to make driving safer.

At the organisation level, we can consider any company that uses AI/ robots in order to produce satisfactory goods for customers. The AI/robots that are used must have similar characteristics to those just mentioned for the self-driving car. It is up to those who organise the production, distribution, and sale of business products to configure the $\mathrm{AI} /$ robots so that they have the ability to contextually relate and evaluate the progress of relationships in various sectors within the company and in the context of sales and of consumption. It is not enough to improve the cognitive intelligence of the single $\mathrm{AI} /$ robot. It is necessary to build $\mathrm{AI} /$ robots that are able to regulate the progress of the network of relations between producers, distributors, and consumers of company products. Is this possible?

I do not know practical examples already in place, but the idea of "distributed responsibility" among all the actors in the network that produces, distributes, and uses the goods produced goes in this line. It requires that the $\mathrm{AI} /$ robots be constructed and monitored within a project of (i) observation of their relational work, (ii) with the ability to diagnose deviations from satisfactory procedures and results, and (iii) the orientation of the management to operate according to a relational guidance program. 


\section{Summary}

With the fourth technological revolution, social identities, relations, and organisations are forced to take shape in the environment of a Digital Matrix that works through a symbolic code that tends to replace the ontological, ideal, moral, and theological matrices that have structured societies in the past. As a form of Technological-Gnosis, its peculiarity is that of making the boundaries between human and non-human labile and crossable in every way in order to foster hybrids. Hybrids, however, are not random and purely contingent entities. They stem from complex interactional networks in which social relations are mediated by the DTM. The processes of hybridisation are selective and stratified according to the ways in which the human/non-human distinction is thought and practised. Three possible scenarios of hybridisation can be outlined: adaptive, turbulent, and relationally steered.

As a criterion for evaluating hybridisation processes, I have proposed assessing how digital technologies mediate the transformations of people's mind-body identities with their sociality so as to assess when such mediations produce those relational goods that lead to a virtuous human fulfilment or, instead, relational evils.

The justification of this perspective is based on the fact that human beings are at the same time the creators of society and its product. They are the parents and the children of society. As a consequence, it is not the physical world (Anthropocene) and/or the artificial world (AI/robots) that can produce the human being as a human being, but society, which, from the point of view of my relational sociology, consists of relationships, that is "is" (and not "has") relationships. Therefore, the quality and causal property of what is properly human comes into existence, emerges and develops only in social life, that is only in and through people's sociability, which is however embedded in the practical order and has roots in the natural order. In fact, only in sociality does nature exist for the human being as a bond with the other human being. The vital element of human reality lies in the relationality, good or bad, that connects Ego to Other and Other to Ego. The proper humanisation of the person is achieved only through the sociality that can be enjoyed by generating those relational goods in which the naturalism of the human being and his technological enhancement complement each other. 


\title{
10 Prospects
}

\author{
Should we abandon the dream \\ of a human(e) society?
}

\begin{abstract}
"What madness not to know how to love men as they are! Fool who cannot bear to be a man" (Augustine of Hippo, Confessions, 4, 7)
\end{abstract}

\section{Can society retain its human qualities and properties?}

The fact that the boundaries between the human and non-human (both natural and artificial) entities evaporate in many social and cultural contexts reveals that the ground is ready for a kind of society (read: networks of social relationships) that abandons its human characters to take on others, still unknown. What features will the future society have?

Many fear the advent of algocracy (the rule of algorithms) as that particular form of governance of relationships where the process is managed by the operating algorithms of digital platforms. According to Aneesh (2002), who invented this term, algocracy tends to flatten all bureaucratic structures because it does not require any intermediate or centralised level of management. Thus, the indestructibility of bureaucratic structures prophesied by Weber seems no longer a stable truth despite (or because of) the continued advance of formal rationality.

In this way, social relations become even more indifferent to differences ("neutral") than they already were in modernity (Aneesh 2015). A real "neutralized society" is born - neutral even in the face of human/non-human difference. People become disconnected from the local interactions and personal relationships that make their lives anything but neutral.

By subjecting social relations to virtual games, and by neutralising relational goods, the possibility of finalising social life towards the emancipation of the human person is brought into play.

Advanced societies seem to be more and more dissatisfied with the limitations that are inherent in the so-called human nature. The premise of this dissatisfaction was clearly expressed by Friedrich Wilhelm Nietzsche in his famous writ "Human, all too human" (1878), in which he launched the theme of transcending the human for the next centuries. Since then, it is a matter of fact that the old Western humanism has declined for many 
reasons that cannot be enumerated here. In principle, this evolutionary trend has to do with a culture that does not accept the limits of the human, which is considered too weak, fragile, and perishable, and therefore should be transcended. As Simmel (1970: 8) has claimed, "man is the limited being without a limit."

Looking at the future horizon, we ask ourselves: is it still possible, if it ever makes sense, to speak of some sort of (neo) humanism or should we abandon humanism and proceed towards the transcendence of the human?

What does it mean to transcend the human? As it is well known, today, this means getting into the post-human, the trans-human, and the cyborg. Well, if we transcend the human, in what society will the next generations live?

Transcending the human entails denying certain characteristics that are uniquely human (including those of weakness and physical limitations) and those that constitute human nature, just as we can still observe them today (Haslam 2006). It implies operations that take something away from the human to replace it with something else.

Giving the human beings special skills through particular technologies can strengthen or threaten human nature, depending on how technologies affect the constitution of the human person and her internal as well as external relationality, and therefore the qualities and properties of society understood as a web of relational social forms.

\section{The two currently prevailing scenarios of societal evolution}

To my view, we have to face two grand scenarios of the societal evolution, underlying the present cultural dynamics in the globalised world, that implicitly contain the two prevailing models of understanding human transcendence. On one side, the Western scenario based upon the expansion of science combined with technology, positivistically geared to generating a new being with superhuman abilities. On the other side, the Eastern idea that the world evolution consists in a continuous bringing into existence what does not exist, that is a process in which human transcendence means the construction of a machine of Nothing.

Western scenario is fundamentally linear or multilinear in the way it considers evolution, while Eastern scenario is basically circular, although in both scenarios, there may be spiral visions.

1 The first scenario assumes that human nature is equal to a bios equipped with psychism, what is human in the future will consist of these dimensions enhanced by technology. The distinctions between human beings and other living beings (animals and plants) will be gradually falling. Human sociability will be transformed accordingly. In this perspective, the human being is considered as a nature's beautiful glitch, that is a beautiful error of the natural evolution that has succeeded 
in surviving and now is evolving into an infosphere which becomes anthropo-eccentric rather than anthropocentric (Floridi 2015). The basic tenet is that "more tech makes us more human." The great vehicle of this epochal transformation is seen especially in the ICTs, most of the times in combination with genetic engineering, nanotechnology, biotechnology, and cognitive sciences. The latter cease to be instrumental means mastered by people and become autonomous forces that modify (i) our self-conception (who we are), (ii) our mutual interactions (how we socialise), (iii) our conception of reality (our metaphysics), and (iv) our interactions with reality (our agency). In each case, ICTs have a huge ethical, legal, and political significance, yet one with which we have begun to come to terms only recently.

The supporters of these views are convinced that the aforementioned impact exercised by ICTs is due to at least four major transformations: (i) the blurring of the distinction between reality and virtuality; (ii) the blurring of the distinction between human, machine, and nature; (iii) the reversal from information scarcity to information abundance; and (iv) the shift from the primacy of stand-alone things, properties, and binary relations, to the primacy of interactions, processes, and networks.

The impact summarised in (1)-(4) and the transformations behind such an impact listed in (i)-(iv) are used as a test to verify the foundations of the Onlife Manifesto philosophy, in the following sense. It is assumed that people's perceptions and understandings of the realities surrounding them are necessarily mediated by concepts. The latter work like interfaces through which people experience, interact with, and semanticise (in the sense of making sense of, and giving meaning to), the world. In conclusion, we humans grasp reality through concepts, so, when reality changes too quickly and dramatically, as it is happening nowadays because of ICTs, we are conceptually wrong-footed (Floridi 2015: 2-3).

My criticism to the technological versions of this Western scenario concerns the fact that there is a lot of determinism in it. In particular, one could observe that, contrary to what this vision maintains, perceptions precede their conceptualisation, so there is a gap in between them the enigma of the relation) and not an overlapping as this scenario supposes. It is true that perceptions appeal immediately to the need for attributing a cultural meaning to what is perceived, but this happens through a reflexive processing of sensory experience. While for relationists, perception is necessarily mediated by concepts, as if they were the unavoidable interfaces through which reality can be experienced, relational sociology argues that cultural mediation is necessary for the full recognition of reality, but it is the product of a process of personal and relational reflexivity, which of course may be very weak or even distorted, and indeed can be of many different kinds. 
It seems to me that opposing this view on the basis of an individualist first-person perspective is not enough, because this view of personhood does not recognise the consistency needs and uniqueness of the relation between mind and body in each person. Baker (2007) and Archer (2020), for example, claim that the first-person perspective is necessary and sufficient to preserve the sense of the human. Their perspectives are perfectly compatible with this evolutionary scenario, once we admit that technology can change the human body without affecting the way one thinks of oneself as oneself. I disagree with these scholars because I believe that denying the essentiality of the mind-body relationship, as well as the importance of social relations, in the constitution of human identity, means abandoning the human to enter another order of reality. Despite an apparent support for the enhancement of the human, they actually hide a reductive understanding of the human and its depreciation in favour of an increase in the importance of technology and the Digital Matrix.

We have to pay attention to the influence exerted by social relations on the first-person perspective. That is why, while I too maintain a first-person perspective (together with the second-person and third-person perspectives), I do not agree with Baker when she thinks that we can split what is human (the animal) and what is a person (in her definition: "self-reflectivity on one self"), as - in her view - they do not form an identity. On the contrary, I argue that the notion of "human person" indicates the more overarching concept that captures the fully social, interconnected, and bonded human being in her entirety. However, much, as Luhmann states, the body seems to disappear from modernity, and, however, much the split between the psychic system (consciousness), the social system and the biological system increases, thus making the unity between these elements more and more difficult, however, as Francis Halsall (2012: 4) writes, "the body has the ability to migrate between different systems and, thus, has a transcendent status in social systems. That is, the body can migrate between social systems and, in Luhmann's terms, irritate them in significant ways."

Human body, mind, and agency cannot be completely separated. Either they are integrated, or the human is no longer such and the action is no longer human. The unity of this difference is a relation. Splitting body and mind is an abstract operation that can serve to give absolute priority to a first-person perspective, defined as "our inwardness - our abilities not just to think, but to think about our thoughts; to see ourselves and each other as subjects; to have rich inner lives" (Baker 2002: 370). This is a naïve perspective. It does not see how the relationships between people influence their inner life and does not provide any meaningful account of the real experiences of "normal" people (not humanoids or robots) in connecting their body and mind in a hyper-technological society. The "inner" abilities Baker is referring to are strictly dependent on the new communicative environment as well as the possibility of keeping a sense of identity in the connections between body and mind. Baker's first-person perspective seems to be a strategy that is proposed to leave the body to the technologies and preserve (indeed enrich) the 
"reflective mind" (Baker 2013, 2016), as if the same technologies did not have a powerful influence on the personhood through body modifications. In the end, what she calls "constitution view" of the human person is a-relational, in a double way: because she splits what is human and what is person (Baker 2002: 370), and because she ignores the role of social relations in constituting the way in which the first-person perspective can actually operate.

2 The second scenario can be illustrated by quoting an evocative paper by Bilimoria (2012), in which he comments on the Veda books, where it is written: "In the beginning, there was neither Non-Being (asat) nor Being (sat)" (RgVeda X.129.1; it is called "hymn to the creation"). Bilimoria asks, "what was there, bottomless deep?" Eventually, he responds positively. The bottomless deep is called "Nothingness" (the absence of existence). The argument is that "Radical Nothingness ought not to be feared: it may have therapeutic value in the hallowed Wittgenstenian-Yogacārin sense (an antidote for excessive linguistic conceptualism); it may prove to be the timely yet fairest watchdog on the excesses of the Technoscience culture (to which Heidegger similarly drew attention), as well on fundamentalisms of all varieties, the return of religion to the public sphere (despite the Enlightenment's decree), secularism re-seeking the sacred enchantments, and on other attachments to Absolutes, perhaps also to being/Being, and an acute partner in inter-faith, intradeist and inter-faithless conversation of the Four Horsemankind" (Bilimoria 2012: 528). "Therefore - so he concludes let there always be non-being so we may see their subtlety, and let there always be being so we may see their outcome. The two are the same. But after they are produced, they have different names. They both may be called deep and profound (...) Deeper and more profound..." (quotation from Tao-te Ching by Lao-tzu) (Bilimoria 2012: 528).

Unlike the progressive vision of the former, this scenario leads to see the evolution of the whole creation, and the human evolution within it, as a "machine that creates Nothingness" (ibid) in the sense of bringing into existence what does not exist originally. It can mean to give real existence to certain fictional beings. In this scenario, there is no reason to distinguish between human and non-human, or to think of transcending the human, simply because, to put it bluntly, the religious matrix of these Oriental cultures is a sort of naturalistic pantheism.

The general notion of "matrix" here refers to a cultural code (symbols and norms for their use) that orients or steers the way in which agents/actors intersect their intents, the situational context in which they find themselves, the meanings they use and the generated effects (Donati 2021). While the term matrix is generally used in the sense of "(symbolic) matrix of society," here I would like to underline its transcendent dimensions (I will focus on the concept of transcendence in the next pages). 
In short, I have chosen to mention these two visions because, although distant and even contradictory in some respects, they seem to merge in the globalising society. What emerges from their mix is a culture that views human transcendence as an endless evolution that is supposed to supersede human deficiencies by mixing natural and artificial elements of all kinds. Western rationalism is mixed with Oriental naturalism within a complex "nature + technology" in which transcendence is a matter of an immanence that surpasses itself. The result is a vision of human transcendence as a process of incessant creation of new singularities (see the theory of technological singularity). From my point of view, however opposite, these two scenarios seem to be connected by a subtle and hidden thread, that of a kind of vitalism mixed with some kind of (a Nietzschean?) nihilism. What now is called "post-truth politics," is, in fact, a form of cultural nihilism, since on the digital media "anything goes," which means that communications do not discriminate between truth and non-truth, and communicative rationality is substituted by emotionality.

Bruno Latour's thought, which refuses the concept of "out there" vs "in there" and renders the distinction between subject and object simply unusable by arguing that there are only "events," " provides an example of such an approach. It blends the Western culture with many typical elements belonging to the Oriental culture. According to Latour, there is no reality out there, everything goes, science has no conceptual content, the more ignorant one is the better, everything is political anyway, subjectivity should be mingled with objectivity, and "action is simply not a property of humans but of an association of actants" (Latour 1999: 182). Following Vandenberghe, we might ask: should we expect to become "humants," that is humans dehumanised by the actant? "When science enters in action, meaningful action disappears and all we are left with is a pasteurized and desymbolized world of strategically acting dehumanized humans, or humants" (Vandenberghe 2002: 55).

\section{A third scenario}

The two scenarios just mentioned place human transcendence, respectively, in the total immanence of the technological evolution and in an immanent process of creation that makes exist what is not. It is noteworthy that the adoption of both these perspectives is part of the strategy adopted by Luhmann to build his sociological theory overtly anti-humanistic.

I would like to present here a third scenario that introduces another idea of human transcendence. It conceives transcendence as an emerging relation between what exists (immanent reality) and what can be (transcendental reality).

1 "There is no object, no subject, no contradiction, no Aufhebung, no mastery, no recapitulation, no spirit, no alienation. But there are events. I never act; I am always slightly surprised by what I do" (Latour 1999: 281). 
I start by offering my own interpretation of the following sentence from the Vedas books: "sages who searched with their heart's thought discovered the existent's kinship in the non-existent." 2 What does it mean that there is a "kinship" between the existent and the non-existent when referred to the human being?

In my view, kinship can be interpreted as a particular relation between the existent and non-existent forms, not necessarily - as the original text seems to suggest - the reversibility of being and non-being within a cosmic vision of the emptiness of all things. Instead, if we adopt a relational perspective, the kinship between Being and Non-Being appears as an enigmatic relation, so that the existence and possible evolution of the human being (and its constitution) could be seen as a particular kind of relationality between immanence and transcendence.

In other words, we can say that, at the beginning (of any creation, not only of the original creation), there is not a bottomless depth, but a Being ever able to transcend itself by relating himself to the Non-Being (what does not exist yet). It is in this process of transcendence (emergence) that being and non-being are related, while no one of them can exist per se in absolute isolation. The very nature of creation would then consist in a relation that brings into existence what does not exist through a process of emergence. Is it possible to think that what is human can transcend itself in this "relational nature" of the creation? The possibility of arguing in favour of a positive answer to this question lies in the social ontology of what we mean by "relation" and "relationality."

The relations between a human being and other living beings (animals and plants) or machines do not possess the same qualities and causal properties of the inter-human relations. If the interpersonal relations are replaced by technological devices, for instance the ICTs or robots, they become digital or mechanical, that is they change completely their qualities and properties. They take on qualities and properties other than human ones. These are, for example, the social relations of the actant theorised by Bruno Latour.

In short, I want to support the thesis that the human does not transcend itself neither in the technological enhancement of the individual nor in the personal unit act, nor in creating more sensitive, smart, and human-like machines, but in the human relationship and only in it. As Anders (2016: 45) claims, "what makes 'thing' the person is not the scientific treatment, but the actual treatment of man by man."

Since I realise that the reader may wonder "what is the mystery of this "magical' relationship," in the following I will tackle the riddle of the social relation. The issue concerns the relationships between what is human and

2 RgVeda X.129.4 (italics mine). Another translation from Sanskrit is "The sages who have searched their hearts with wisdom know that which is kin to that which is not." 
all its environments (in the sense of systems theory), that is both the physical and the transcendental realities.

\section{The distinction human/non-human is a transcendental relation containing an enigma}

To speak of transcendence implies a social ontology of reality that, in turn, refers to a transcendental reality, and therefore it calls for the existence of a transcendental cultural matrix to which the ontology is closely connected. When I claim that this matrix is "theological," I do not refer to the dogmatic beliefs of a specific religious faith, but I mean the symbolic code underlying every great culture or civilisation concerned with ultimate realities. Every scientific theory depends on a cultural matrix through which it "sees" the world, and this matrix has meta-reality presuppositions of any kind. The founding fathers of sociology, like Comte, Marx, Durkheim, and Weber, provide different examples, and also today we can find such an underlying matrix in every sociological theory, although it is often unspoken, hidden, and removed.

The service of the transcendental matrix is to mould our needs, experiences, and ways of thinking. It does this through an operation with which it treats the existent (the Dasein) in the light of what it supposes to transcend it. What is this operation of transcending what is simply observed at a certain moment?

Transcendence here can mean:

1 a metalevel of reality that we must and can reach, in order to explain what happens in reality, by relating to a further "layer" beyond what already exists in an open horizon (matrix) of up-grading orders of realities;

2 the necessity of this meta-relation, as distinct from its contingencies;

3 whereas "necessity" is a matter of recognition of what is latent (hidden, invisible, unmeasurable) as an "ultimate reality" bordering on religion (i.e. what is sacred, and, as such, conferring a dignity to every being, in the sense of recognising that every existing being deserves the respect that is due to it according to its own nature).

The recognition of the dignity of every entity (in Latin the word dignus means "a thing or a person deserving respect for its qualities") marks the boundary between what I call "immanent transcendence" (as a product of society) and "transcendent transcendence" (as a reality which is not the product of society, because it is pre- and meta-social).

The problem with which we are confronted is: what are the relationships between the human and the transcendental realities?

The possibility of linking human relations and transcendental relations depends on the fact of being able to see and manage the enigma of the relation as such, the enigma that lies in relationality as such (Donati 2015c). 
The enigma can be simply expressed with this question: why in order to achieve what people love and desire, transcending their constraints and limitations, must they go through the relations with other people especially when new technologies are available that make human relationships unnecessary?

Take the example of Sweden. As Erik Gandini's docu-essay The Swedish Theory of Love (movie 2015) has shown, Swedes' obsession with independence and self-sufficiency, coupled with a welfare state that provides for every physical need, has produced a lonely and alienated society (it's called "statist individualism"). Women can have a baby alone without a sexual relationship with a male partner, sperm-bank donors abound, many people die alone, and state investigators try to find the next of kin of old people who died alone and a suicide who were not found for years because all their standing orders were paid automatically. It is no coincidence that many young people refuse to live in the world of high technologies and organise camping in the woods to make alternative life experiences in common, take care of each other, relate and be in physical contact between people and with nature.

Why human beings should transcend their limitations and constraints by avoiding dealing with other people, especially when technologies can replace the presence of other human beings? If we know that a particular gene produces aggressive and deviant behaviours, why not avoid having to deal with these relationships by genetic engineering on DNA? Today, many jobs and services can be performed by machines: should we look for smart AI robots or humanoids who can work better than humans, by this way avoiding any sort of those interpersonal relations that we know in advance will be deficient and vexatious?

I am thinking of ordinary life, where people rely on technologies that once required human relations. The final horizon is that of a society, as Marx and Engels (Engels 1959: 386-387) advocated, in which takes place "the administration of things by things." Of course, they could not speak of robots and cyborgs. We have done a lot of progresses ever since. Today, a-normative regulations justified by an adiaphoric ethics are amongst those procedural devices that allow present and future social systems to go in that direction.

The modern West has exalted the Subject (the $I$ ) at the expense of its relations. It thought it could provide social relations at its pleasure. It also thought and thinks in system terms, with the "system" being understood as instruments of liberation of individuals. It refused to answer that which I call the enigma of the relation (Donati 2015c), which consists in the fact that Ego needs an Other who is the source at the same time of support and conflict.

Ego must find something in common with the Other and at the same time assert the differences with him. When modernity sought to give an answer to this riddle, it created new problems, either because the answer was that of conflict, of division, of confrontation, or stipulated and then betrayed contracts, or because it pursued strategic games that have ended badly. The post-human exacerbates these problems because, while providing more advanced technologies to Ego, it further removes or dehumanise social relations. 
The point is that Western culture does not have devices (indeed a cultural matrix) capable of treating the riddle of the relationship, in the sense of knowing how to connect with other people so to have something in common while accepting the inevitable distress arising from differences. From Schelling and Hegel forward, there have been many attempts to think about this issue. Aside from countless authors from the 20th century (especially Foucault and Deleuze), it is sufficient to cite Derrida (1982), who sees the difference as ambiguity, and Lyotard (1984), who proposes analysing les différends qui nous opposent through a radical relationism. The point is that, in all philosophy and culture that starts from the idealism of the 18th and 19th centuries and into the present day, the possibilities of conceiving of a relational matrix of society are still missing.

In the end, Western modernity has removed the reality - that is the proper space-time - of social relations, in order to create an indefinite number of relations, all virtual, all otherwise possible, in order to be able to play with them. Are these, as Jean Baudrillard says, "fatal strategies"? Or, are they functional strategies, as Luhmann says?

Luhmann's explanation, not surprisingly, goes back to Greek mythology. The solution of the enigma of the relation comes to be represented by Perseus, who managed to survive because he adopted a precise relationist strategy, consisting in avoiding the deadly gaze of the Gorgons (the enigma of the relation) through a continuous change in his position, in such a way as to never directly cross the gaze of the Gorgon. Luhmann (1990) calls this strategy "eurialistics," according to which the insoluble problems of society (its paradoxes, like its enigmas) should be treated in such a way as to avoid directly confronting them, which means constantly changing one's point of view by rendering everything "relative," that is, adopting a radical relativism, or rather radical relationism. It is not surprising that the prevalent culture of today recommends precisely this way of life as the solution to the enigma of the relation: it is that way of relating to others that consists in believing that one cannot and should not have any certainty in the face of problems.

Consequently, our ways of life are in conflict with an increasingly radical crisis, made of existential and solipsistic voids, because we are not able to confront and to respond to the enigmas of human relationships that ask to go beyond appearances. It is precisely here that lies the need to transcend the human in its present limitations.

\section{Any humanism requires a certain social ontology of relationality}

Affirming the transcendence of social relationships does not mean denying their immanence. This affirmation must be clarified through a confrontation between substantialist, relationist, and relation $a l$ sociologies.

Both the relational and relationist approaches share the need to overcome the substantialist approaches of various types (spiritualist, naive realist, 
positivist, rationalist) - which have dominated scientific thought in the past. This lesson comes to us from quantum theory which has questioned the existence of "the thing itself" (Rovelli 2020).

I would like to clarify my point of view by saying that the relationist point of view goes too far: it thinks the macro level of reality as if it could be understood in the same way as the micro level in which the indeterminacy principle applies. It conflates the micro and macro levels because it does not see the meso level. In this way, it does not understand the making of reality. Table 10.1 outlines a comparison between substantialist, relationist, and relational approaches with regard to social reality.

I refrain from commenting on it in detail because it seems clear enough to me. I just want to point out the most fundamental differences. Social ontology, paradigmatic assumptions, and epistemologies are basically different. Therefore, radical differences follow in the way of understanding the levels of reality, social structures, and social agents, what distinguishes the human and its capacity for transcendence.

The substantialist approach remains tied to the idea that an entity is real because it exists "in itself," regardless of the relational context, and exerts effects which are consequences of its own substance. The relationist approach completely overturns the previous way of observing reality. It assumes that an entity is real because it is observed and thought of as such by actors who are situated in highly contingent contexts. In short, something is real because it is observed by an observer who according to his tools of knowledge, believes it to be real. Contrary to both the first and the second, the relational approach thinks instead that an entity is real because it has specific causal powers in itself due to its own structure which is, however, formed by the dynamics of the past and present relational social context.

For relationists, social relations are nothing but transactions that occur in different social situations. They treat relations like a continuous and undetermined reference to infinity (an eschaton). Rorty (1999: 54) clarifies it very well, when he affirms, "Everything that can serve as a term of relation can be dissolved into another set of relations, and so on forever. There are, so to speak, relations all the way down, all the way up, and all the way out in every direction; you never reach something which is not just one more nexus of relations."

Is that precisely how things are? I think not. Relationality does not cancel out substances, but forges them in social time, so that we must always read reality as consisting of substance and relation as co-principles of what exists or that there may exist (the "being").

For my relational approach, the terms of the relation cannot be dissolved in other sets of relations by reason of the reality of their concrete relation, which is a structure with its own emergent properties and causal powers.

Open is the question of what social ontology we need in order to support a relational (not relationist) interpretation of reality, and what kind of transcendental matrix it needs. We find here two opposite positions. 
Table 10.1 A comparison between substantialist, relationist, and relational approaches

\begin{tabular}{|c|c|c|c|}
\hline $\begin{array}{l}\text { Features of each } \\
\text { approach }\end{array}$ & $\begin{array}{l}\text { Substantialist } \\
\text { approaches }\end{array}$ & $\begin{array}{l}\text { Relationist } \\
\text { approaches }\end{array}$ & $\begin{array}{l}\text { Relational } \\
\text { approaches }\end{array}$ \\
\hline $\begin{array}{l}\text { Social ontology } \\
\text { (something is } \\
\text { real because...) }\end{array}$ & $\begin{array}{l}\text { An entity is real } \\
\text { because it exists } \\
\text { "in itself," } \\
\text { regardless of the } \\
\text { relational context, } \\
\text { and exerts effects } \\
\text { which are } \\
\text { consequences of its } \\
\text { own substance }\end{array}$ & $\begin{array}{l}\text { An entity is real } \\
\text { because it is } \\
\text { observed and } \\
\text { thought of as such } \\
\text { by situated actors, } \\
\text { that is because it is } \\
\text { believed as real }\end{array}$ & $\begin{array}{l}\text { An entity is real because } \\
\text { it has specific causal } \\
\text { powers in itself due to } \\
\text { its own structure which } \\
\text { is however formed by } \\
\text { the dynamics of the } \\
\text { relational social context }\end{array}$ \\
\hline $\begin{array}{l}\text { Paradigmatic } \\
\text { assumptions of } \\
\text { theories }\end{array}$ & $\begin{array}{l}\text { Naïve realism/ } \\
\text { positivism } \\
\text { (substances } \\
\text { determine social } \\
\text { relations) }\end{array}$ & $\begin{array}{l}\text { Radical } \\
\text { constructivism } \\
\text { (relations determine } \\
\text { the entities that are } \\
\text { in relation and } \\
\text { therefore their } \\
\text { "substance") }\end{array}$ & $\begin{array}{l}\text { Critical, analytical, and } \\
\text { relational realism } \\
\text { (substances and } \\
\text { relations are } \\
\text { co-principles of social } \\
\text { reality) }\end{array}$ \\
\hline $\begin{array}{l}\text { Epistemological } \\
\text { presuppositions }\end{array}$ & $\begin{array}{l}\text { Knowledge consists } \\
\text { in the subject's } \\
\text { ability to adapt to } \\
\text { the object }\end{array}$ & $\begin{array}{c}\text { Knowledge of reality } \\
\text { is the observation } \\
\text { of the observer } \\
\text { who determines the } \\
\text { space-time of the } \\
\text { object }\end{array}$ & $\begin{array}{l}\text { Knowledge consists in the } \\
\text { observer's relational gaze } \\
\text { on reality, that is as an } \\
\text { activity mediated by the } \\
\text { relationships between } \\
\text { knower and known }\end{array}$ \\
\hline $\begin{array}{l}\text { The levels of } \\
\text { reality }\end{array}$ & $\begin{array}{l}\text { It is assumed there } \\
\text { is an analogical } \\
\text { correspondence } \\
\text { between the micro } \\
\text { and the macro } \\
\text { levels }\end{array}$ & $\begin{array}{l}\text { The micro and } \\
\text { macro levels are } \\
\text { conflated }\end{array}$ & $\begin{array}{l}\text { Micro and macro are } \\
\text { mediated by the meso } \\
\text { level which transforms } \\
\text { the indetermination of } \\
\text { the micro level into } \\
\text { determination of the } \\
\text { macro level }\end{array}$ \\
\hline $\begin{array}{c}\text { Social agent } \\
\text { /actor is ... }\end{array}$ & $\begin{array}{l}\text { Individual substance } \\
\text { of a rational nature }\end{array}$ & Actant & Relational subject \\
\hline $\begin{array}{c}\text { Social structures } \\
\text { are ... }\end{array}$ & $\begin{array}{c}\text { Expressions of } \\
\text { human nature } \\
\text { cultivated through } \\
\text { cultural } \\
\text { developments }\end{array}$ & $\begin{array}{c}\text { Stabilised } \\
\text { expectations based } \\
\text { on past } \\
\text { communications } \\
\text { and transactions }\end{array}$ & $\begin{array}{l}\text { Emergent effect of past } \\
\text { actions, interactions, and } \\
\text { transactions having their } \\
\text { own sui generis reality }\end{array}$ \\
\hline $\begin{array}{c}\text { What } \\
\text { distinguishes } \\
\text { the human } \\
\text { being }\end{array}$ & Rationality & $\begin{array}{l}\text { Meanings in } \\
\text { communication }\end{array}$ & Reflexive relationality \\
\hline $\begin{array}{c}\text { The human being } \\
\text { transcends his/ } \\
\text { herself in } \\
\text { society as ... }\end{array}$ & $\begin{array}{l}\ldots \text { s/he is able to } \\
\text { express his/her } \\
\text { potential in the } \\
\text { perfection of } \\
\text { acting (virtuosity } \\
\text { of her act) }\end{array}$ & $\begin{array}{c}\ldots \text { s/he hybridises } \\
\text { with the natural } \\
\text { and artificial } \\
\text { environment, } \\
\text { adapting to a } \\
\text { naturalistic } \\
\text { evolution }\end{array}$ & $\begin{array}{l}\ldots \mathrm{s} / \text { he flourishes in } \\
\text { accordance with the } \\
\text { potential of his/her } \\
\text { species-specific } \\
\text { relational nature } \\
\text { (relational essentialism) }\end{array}$ \\
\hline
\end{tabular}

Source: Author. 
On one side, there are those who argue that relationality is constituted and intelligible without any need for a transcendental matrix. Along these lines is Niklas Luhmann, who uses an autopoietic and self-referential cultural matrix for which relationships serve to operationally close each system, and simultaneously as a cognitive opening of each system. What unites him with the relationists is the use of a holistic formula of total immanence of social processes, according to which relational emergence excludes the reference to a transcendental reality. According to this theory, we must consider that, for example, a social cooperative, a family, a voluntary association, or a social movement - as a specific relational structure - is formed from itself without the need for an explanation that refers to some transcendental principle.

That is why, I disagree with Jean-Sébastien Guy (2018) when he tries to credit the thesis that Luhmann can be considered as a sort of forerunner of relational sociology. Guy writes, "system operations for Luhmann are relational operations. Indeed, with each operation of communication, a unity is projected over different elements scattered in time and space. These elements are as many micro-events that are connected together so as to create the macro-event that communication is (or meta-event: an event made out of other events). Since communication means coordination, this is accomplished when individuals take their cues from each other so that what one individual did at time $t$ can be selected retroactively by another individual as the reason or justification for his own action at time $\mathrm{t}+1$.These different elements are connected together so as to create a unity out of them that the communication process (that is, the social system) can then adopt as its own unity" (Guy 2018: 867). Since for Luhmann "relational operations" are defined by a system of cybernetic operations, they have nothing to do with social relationships as we observe them in real life. The above definition given by Guy resembles Weber's definition of the social relation, which I have already commented upon as a reductive definition limited to a communication reference (refero). As a matter of fact, Luhmann explicitly denied that the concept of social relation could be considered the basic unit of sociological analysis (this statement was made by conversing with me and is explained in a footnote of one of his last books commenting upon my Teoria relazionale della società). ${ }^{3}$

3 "Es gibt nach wie vor gute Gründe für die Beibehaltung dieser Begriffe [Struktur, ndr], wenn es darum geht, Systemmodellezu beschreiben. Aber über Modellbildung kommt man damit nicht hinaus. In ihrer operativen Wirklichkeit und in der Fluidität vor allem auch: im Reichtum ihrerübergangenen Möglichkeiten sind Systeme sehr viel komplexer, als es in einem Modell gezeigt werdenkann. Deshalb vermag ich auch dem Vorschlag von Pierpaolo Donati, Teoria relazionale della società, Milano 1991, nicht zu folgen, die Systemtheorie durch eine Relationentheorie zu ersetzen..." [translation: "there are still good reasons to keep these terms [like structure] when describing system models. But you can't go beyond modeling. Above all, in their operational reality and fluidity: in the richness of their past possibilities, the systems are much more complex than those that can be shown in a model. Therefore, I cannot follow the proposal of Pierpaolo Donati, Relational theory of society, Milan 1991, to replace the theory of systems with a theory of relations...”] (N. Luhmann, Die Gesellschaft der Gesellschaft, Suhrkamp. Frankfurt a.M., 1997, vol. 2, p. 34, footnote 95). 
A different view is that of relational sociology, for which relation - as ontological entity (insofar as it is) - has its reason for being in a transcendental reality (as defined earlier). This means that nothing comes into existence if not through/for/with the relation that possesses in itself a principle of transcendence. For example, the reality of a social cooperative, a family, a charity, a social movement, a company, a state, as a relational phenomenon sui generis - regardless of different contingent historical forms that these realities can take on - is fully comprehensible in its "being" only in the light of a transcendental symbolic code.

In these two seemingly opposed perspectives, Luhmannian and relational, there is the enigma of the relation that has come down to us through history. The riddle to be solved is this: is social relation in the world self-generated, as orthodox Luhmannians say, or does it depend on a "formula of transcendence"4 as relational sociology maintains?

The answer is not simple. In a certain way, it is tempting to say that both perspectives contain something true, but obviously not on the same level. If we place ourselves before the comparison of these two positions (claiming that social relation is formed even if transcendence does not exist and claiming, instead, that, if transcendence is not given, relation - ontologically speaking does not come to exist), and we ask ourselves who is right, we could respond by saying that they are both right, but from totally different points of view.

The former (the "Luhmannians") are right in the sense that it is true that relations form "spontaneously" (due to interactions and inherent operations in the same contingent relations), and nonetheless, from a critical point of view, it is by no means assured that they are positive and humanising relations, but rather the opposite is quite likely. For Luhmann, in fact, the relation is certainly an emergent phenomenon, but completely contingent and devoid of structured distinctions rooted in moral values inherent to relations themselves. That is why, Luhmann formulates an anti-humanist sociology.

The others (those who refer to a transcendental symbolic matrix) are right in observing that an emergent dynamic rooted in a transcendent reality leads more easily to the creation of relational goods that a purely immanent way does not manage to create. This statement can be confirmed by empirical evidence, when, for instance, sociological surveys (Mahoney 2010) point out that families who are inspired by religious values produce more relational goods than those more secularised, although obviously any determinism is always excluded, since no family, even the most religiously inspired, is exempt from producing relational evils.

I propose adopting an approach that links the two perspectives as one sociological thesis to be verified. In short, the thesis to prove becomes that the social relation has its dynamic autonomy on the level of the "second" causes in the sociological sense ("be the second" here means that refer to 
the contingent empirical causes that unfold in space-time, i.e. in each historically situated context), but does not have an absolute dynamic on the level of "first" causes in the sociological sense ("being first" here means that inhere in the ontological potentiality of an entity in coming to emergence as a reality sui generis). This is why, a transcendental matrix is needed in order to recognise human rights beyond contingencies. Human rights, differently from the civil, political, and social rights, are recognised to the individual person in so far as they involve specific relations (for instance every child has the human right to be raised in a family setting instead of an institution), which means that specific social relations should have a transcendental sense to the individual.

What brings together the first and second causes is the "vital relation," which is both human and social because it relates the human and the social to each other. It "stands outside" of terms that it gathers, with its own qualities and causal powers.

The vital relation is the relationship that a human person needs to find outside herself in order to be reflexive in herself on herself, so that she can tell herself to be herself, on the basis of distinctions with what she is not, because this relationship allows her to find her deepest identity. It is the relationship that makes us say: "Now I become myself."

This new relation to oneself is a new (or renewed) belonging to oneself, but beware: this belonging is not an end in itself, otherwise, one falls into solipsism and narcissism. It is a belonging to oneself because, as a relationship, it implies the passage from a non-being (a possibility $\mathrm{X}$, usually undetermined) to being someone (not something) in a reference system (refero) that keeps me bound to other than me (religo). Therefore, it is an emerging relationship (creative) that makes me live unlike in the past on the basis of another reference system that I have chosen and to which I am bound, even if only temporarily.

It is in relating to the outside of her interior life that the person activates the inner self, which exists in itself. Personhood matures through social relations. Personal identity is constituted in, and sustained through, our relations with others, such that were we to erase our relations with our significant others we would also erase the conditions of our self-identity.

The inner life has its autonomy, but this autonomy can only be activated and managed in relation to the outside, that is to significant others and to the social context. For this reason, I call it "vital relationship" (already illustrated in Chapter 8).

This relationality is not only that of symbolic references but includes structural bonds, and for this reason it is a concrete reality and not purely communicative. If the relationality between agents/actors consists of pure contingent communicative events, then it is equivalent to simple interactivity.

The solution to the enigma of the relation rests in the fact that the constitution of a relation according to a certain structure is necessary to ensure 
that each term (agent/actor) of the relation may obtain from it what it could not obtain in any other way. There are no functional equivalents for relations when they have a reality (relationality) sui generis. This is very clear for many traditional pre-modern cultures, like the Chinese culture of Guanxi (which means "relation"), in which any deal or exchange must take place within an established relationship and should lead to a renewed structured relation. It may become a practice also in trans-modern societies, although in different modes.

The non-fungibility of social relations unfolds when people realise that to live in a world of relations thought as "always possible otherwise" turns out to be a dream devoid of reality that generates frustrations, disillusions, addiction, estrangement, dissatisfaction, unhappiness, and a lot of social and psychological pathologies.

People know that certain social goods cannot be achieved through relationships that are standardised or interchangeable at will. Certainly, most people think that certain social relations do not make any difference. Take the case of the belief that all kinds of love relations with human beings or animals or things are equivalent or make no difference, on the basis of the idea that to make a difference between them is an intolerable discrimination. Most times, practical life undertakes to reveal to them the inconsistency of their belief. Then, and only then, they realise that one type of relationship is not fungible with a seemingly similar relationship. They start to think how to solve the enigma by looking for a unique (human) relationship that has no equivalent.

\section{When a social form can be called human?}

Human life can achieve as much as it can of happiness provided it passes through appropriate social forms, that is relationally valid to generate and express the humus of the human flourish. A social formation can be called human to the extent that the nature of its internal as well as external relationality is qualified by the recognition and satisfaction of basic human needs and nourishes people's reflexivity in order to help them to realise their ethical ultimate concerns as a way of transcending human limitations.

In my relational language, a social form is human insofar as the social relationships of which it consists are produced by subjects who are oriented to each other according to a suprafunctional sense. This is precisely what is lacking in the relationships triggered by a robot or an actant.

When I speak of the nature of the relation, I mean its internal principle of operation. It is this operative principle that justifies the assertion according to which "at the beginning [of each reality] there is the relation." This affirmation coincides with the ontological principle that is at the base of relational sociology. It is in this principle, on it, with or without it, which is played the fate of Western reason, and therefore of the "Western society" itself, as regards a possible new humanism. 
Relational sociology consists in the observation that society, or rather, whatever phenomenon or social formation (a trading company, a civil association, the state), including the global society, is neither an idea (or a representation or a mental reality) nor a material thing (biological, physical, technical, economical, or other types), but a complex network of social relations.

It is neither a "system," more or less preordained or impinging upon individual agents, nor a product of individual actions aggregated or added together, but it is another order of reality: the society is relation, and every social formation - think also of the Internet - is made of social relations. Every society or social form is distinguished by the mode sui generis of combining elements that make up what I call its constitutive "social molecule," whereas such elements interact according to certain relational dynamics making a new structure emerge which can simply reproduce (morphostasis) or modify itself in a meaningful way in time (morphogenesis).

At the base of this interpretation of the social stands the fact (not the idea or the mere figuration or fiction) that the social relation must be conceived not as a reality that is accidental, secondary, or derived from other entities (individuals or systems), but rather as a reality of its own kind. Such a reality is endowed with an autonomy that consists in the peculiar way in which the affective, cognitive, and symbolic elements proper to human beings come to be combined. To claim that society, indeed the whole of human reality, is relational can seem almost obvious, but it is not at all where the affirmation is understood as an ontological and epistemological presupposition, and therefore one is conscious of the enormous implications that derive from it.

\section{In the end: confronting the dissolution of humanity with relational thinking}

Post-humans and trans-humans are dreams populated by phantoms, and very peculiar phantoms that appear to be real, or better seem to be real because they produce some realities. As Günther Anders (2016: Section 24) claimed many years ago, "Phantoms are not only matrices of the experience of the world, but the world itself. The real as reproduction of its reproductions." The kind of evolution that they feed is far from human, call it dis-human, inhuman, or a-human. To avoid all the deceits that they bring with them, we need to overcome the dissolution of humanity with relational thinking.

Is that neo-humanism? I do not know. What I am suggesting is that we are forced to abandon the old humanism of the West taking into account the deep changes of our times that are trying to transform the human in something else through technological progresses. This fact obliges us to overcome the "personalistic views" of the past that have put what is human inside the individual as such rather than in its intrinsic relational constitution.

Traditional personalism distinguished humans from other living beings for their ability to exercise an inner reflexivity and make individual choices 
in the assumption that the goodness of these features would bring spontaneously the common good. A relational humanism must acknowledge that this old concept of person is no longer sufficient to identify the human person. In the new scenario, a person is human, and becomes more human (i.e. transcends itself), if and to the extent that she generates social relationships that support the flourishing of relational goods from which she feeds herself in order to be more humane.

When we do not know what to do with others and with the situations of life, or what relation to have with the contingent world around us, then we feel confused, weak, fragile, sad, and in crisis. Every existential situation in which we find ourselves, each encounter with something or someone that puts us in trouble, is a relation that challenges us. Usually, we do not think of these situations in terms of relations, because we only see individuals and things. We must confront something (a situation) or someone (people around) and ask ourselves what to do. The fastest way to go is to find a technological device that can solve this problem. In reality, however, behind the challenge of situations, there is a challenge that we do not see, and it is the most important challenge. It is the challenge of the relation in play, which asks us to transcend ourselves.

We need to see the Vital Relation that, by linking the unity of body and mind to the context of existence, makes it possible for us to transcend ourselves. We must learn to deal with this Vital Relation. The enigma to be solved lies in this relation, not in technological devices. Human life is in the enigma of the relation. The enigma of the relation contains the meaning of human life.

My answer to the question whether or not we can be humanist, and practise a humanist sociology, is therefore the following. The crisis of human relations brought about by the modernising and globalising processes is what fuels the search for a posthumanism that can remedy this crisis. The concomitant crisis of a collective faith in something that transcends ourselves, so to become demotivated to pursue a common endeavour, and of interpersonal relations at the micro level finds an explanation in the fact that in both of these relations, the texture of relationality is made evanescent. The loss of the inherent sense of the relation is the "intervening factor" which explains the crisis of social solidarity in its manifold forms. If the sense of the human relation diminishes, then so does the transcendental relation, and vice versa. The same holds true for the relation between the human and non-human realms. For a deeper understanding of this point, it is necessary to recall that the source of the meaning of the relation lies in its transcendental cultural matrix, although the social relation is generated (or is not generated) due to autonomous causes.

In summary, to grasp the why, where, and how the human can transcend itself, it is necessary to "see" the peculiarities of interpersonal relations and their enigmas. One could observe that even the relations with robots are enigmatic, but these enigmas are games, not the stakes of serious life 
(the Durkheimian "vie serieuse"). Thinking that the human person is distinguished from animals, plants, and machines because it is able to think in first person (i.e. to think of oneself as oneself) is not enough. Nobody can exclude the possibility that, in the future, very smart robots or cyborgs could become able to behave in such a way.

To my mind, what distinguishes the human person is her "structural relational constitution," in inner as well as exterior life. This dynamic constitution is the relational substance that enables the human person to transcend herself in the relationship with others and with the world, acting reflexively, not only in herself (personal reflexivity), but on the social relations as such (relational reflexivity), which she assesses as good or evil, taking into account the behaviour of significant others and the reference to the situational context.

The technologies that lead humanity towards the post-/trans-human must be analysed and evaluated based on the criteria of what human relations they assume, and of those they produce.

Early modernity modified natural relationships by creating an acquisitive capitalist society in which relationships are thought of as the fruit of individual choices. Homo faber is held to be master of his destiny. Modernity thus created a historical process in which relationships became a game, an expression of one's emotions and concerns, a play of fictitious distinctions to be used at will. The latest modernity has taken on the task of completely opening Pandora's box of relations, and consequently has met its own failure signalled by increasingly fierce system crises that leave society completely disoriented and uncertain, prey to the most unthinkable risks.

At this point, modernity can continue to play with Pandora's box of virtual relationships that do not respond to human needs, creating processes of neo- and hyper-modernisation, or it can take another path. The latter is the way of a society which, without rejecting the best acquisitions of the past, redefines its own cultural and social structures grounding on a relational social ontology and a relational anthropology. The latter presuppose a way of thinking that knows how to properly see and manage social and human relationships, which means revising the assumptions that the individual is a self-determining being and that social institutions must serve his/her self-determination.

Here is the point where relational society encounters a decisive fracture, in some ways unbridgeable, with all modernity. The fracture lies in the fact of having to take note that, pushing on the self-determination of the individual, we lose the human subject. Who is no longer master of herself, nor of the relational games she can play. The human subject cannot live without meaningful relationships, although their meaning is all to be discovered. Just the loss of significant relationships reveals that the human being is realised not in self-determination or in the technology of doing, but in the encounter with the Other, when the encounter produces a relational good. The being of the person, like that of society, is in the relationship. This is the social 
ontology needed by a new society. This is why, we cannot think of a society that completely expels the human from the social. The human person can be happy only when she knows how to emerge from relationships to the Other and at the same time knows how to "surrender" to these relationships.

However, we are all aware that this transition from modernity to an aftermodernity is not at all obvious. It is open to a sea of alternative possibilities, as I have tried to clarify by talking about the different morphogenetic scenarios.

Wondering whether aftermodern society will be better or worse than modern society makes no sense, because comparison is not possible. The society I speak of tries to transcend the modern one with a relational thinking that remains open to many and different possibilities. The relational society that lies in our horizon is not necessarily good or bad in itself. It can be both. In this, it reveals that it is still "human." 


\section{References}

Adams, M. (2006). 'Hybridising Habitus and Reflexivity: Towards an Understanding of Contemporary Identity?' Sociology 40(3): 511-528.

Alexander, J.C. (1983). The Modern Reconstruction of Classical Thought: Talcott Parsons. London: Routledge and Kegan Paul.

Alexander, J.C. (1994). 'Modern, Anti, Post, and Neo: How Social Theories Have Tried to Understand the 'New World' of 'Our Time.' Zeitschrift für Soziologie 23(3): 165-197.

Anders, G. (2016). Prometheanism: Technology, Digital Culture and Human Obsolescence [1956]. New York, NY: Rowman \& Littlefield.

Andersen, T. (ed) (1991). The Reflecting Team: Dialogues and Dialogues About the Dialogues. New York, NY: W. W. Norton.

Aneesh, A. (2002). Technologically Coded Authority: The Post-Industrial Decline in Bureaucratic Hierarchies. Conference paper, published on ResearchGate (accessed April 17, 2020).

Aneesh, A. (2015). Neutral Accent. How Language, Labor, and Life Become Global. Durham: Duke University Press.

Archer, M.S. (1988). Culture and Agency. The Place of Culture in Social Theory. Cambridge: CUP.

Archer, M.S. (1995). Realist Social Theory: The Morphogenetic Approach. Cambridge: CUP.

Archer, M.S. (2000). Being Human. The Problem of Agency. Cambridge: CUP.

Archer, M.S. (2003). Structure, Agency and the Internal Conversation. Cambridge: CUP.

Archer, M.S. (2007). Making Our Way Through the World: Human Reflexivity and Social Mobility. Cambridge: CUP.

Archer, M.S. (2010). 'Routine, Reflexivity, and Realism.' Sociological Theory 28(3): 272-303.

Archer, M.S. (2013). 'Social Morphogenesis and the Prospects of Morphogenetic Society.' In: M.S. Archer (ed). Social Morphogenesis. New York, NY: Springer: $1-22$.

Archer, M.S. (2016). 'Anormative Social Regulation: The Attempt to Cope with Social Morphogenesis.' In: M.S. Archer (ed). Morphogenesis and the Crisis of Normativity. New York, NY: Springer: 141-168.

Archer, M.S. (2019a). 'Bodies, Persons and Human Enhancement: Why These Distinctions Matter.' In: I. Al-Amoudi and J. Morgan (eds). Realist Responses to Post-Human Society: Ex Machina. Abingdon: Routledge: 10-32. 


\section{References}

Archer, M.S. (2019b). 'Varieties of Relational Social Theory.' In: P. Donati, A. Malo, and G. Maspero (eds). Social Science, Philosophy and Theology in Dialogue: A Relational Perspective. Abingdon: Routledge: viii-xxvii.

Archer, M.S. (2020). 'Can Human Beings and AI Robots Be Friends?' In: J. von Braun et al. (eds). Robotics, AI and Humanity; Science, Ethics, and policy. Dordrecht: Springer.

Archer, M.S. (ed). (2013). Social Morphogenesis. New York, NY: Springer.

Aspers, P., Kohl, S. (2013). 'Heidegger and Socio-Ontology: A Sociological Reading.' Journal of Classical Sociology 13(4): 487-508.

Ahuja, G. (2000). 'Collaboration Networks, Structural Holes, and Innovation: A Longitudinal Study.' Administrative Science Quarterly 45(3): 425-455.

Avnon, D. (1993). 'The Living Center of Martin Buber's Political Theory.' Political Theory 21(1): 55-77.

Baker, L.R. (2000). Persons and Bodies: A Constitution View. Cambridge: CUP.

Baker, L.R. (2002). 'The Ontological Status of Persons.' Philosophy and Phenomenological Research 65: 370-388.

Baker, L.R. (2007). 'Naturalism and the First-Person Perspective.' In: G. Gasser (ed). How Successful is Naturalism?. Frankfurt: Ontos-Verlag: 203-226.

Baker, L.R. (2013). 'Technology and the Future of Persons.' The Monist 96: 37-53.

Baker, L.R. (2015). 'Human Persons as Social Entities.' Journal of Social Ontology 1(1): 77-87.

Baker, L.R. (2016). 'Making Sense of Ourselves: Self-Narratives and Personal Identity.' Phenomenology and the Cognitive Sciences 15: 7-15.

Bateson, G. (1972). Steps to An Ecology of Mind. Chicago, IL: The University of Chicago Press.

Bauman, Z. (1993). Postmodern Ethics. Oxford: Blackwell.

Bauman, Z. (1998). 'What Prospects of Morality in Times of Uncertainty?' Theory Culture \& Society 15(1): 11-22.

Bauwens, M. (2008). 'Par Cum Pari. Notes on the Horizontality of Peer to Peer Relationships in the Context of the Verticality of a Hierarchy of Values.' In: M.S. Archer and P. Donati (eds). Pursuing the Common Good: How Solidarity and Subsidiarity Can Work Together. Rome: Vatican Press: 247-262.

Beck, U. (1992). Risk Society. Towards a New Modernity. London: Sage.

Beck, U., Beck-Gernsheim, E. (2004). 'Families in a Runaway World.' In: J. Scott, J. Treas, and M. Richards (eds). The Blackwell Companion to the Sociology of Families. Oxford: Blackwell: 499-514.

Beck, U., Bonss, W., Lau, C. (2003). 'The Theory of Reflexive Modernisation. Problematic, Hypotheses and Research Program.' Theory, Culture \& Society 20(2): 1-33.

Beck, U., Giddens, A., Lash, S. (1994). Reflexive Modernisation. Cambridge: Polity Press.

Beckert, J. (2006). 'Communitarianism.' In: International Encyclopedia of Economic Sociology. London: Routledge.

Bekoff, M. and Pierce, J. (2009). Wild Justice. The Moral Lives of Animals. Chicago: The University of Chicago Press.

Berger, P.L., Luckmann, T. (1995). Modernity, Pluralism and the Crisis of Meaning: The Orientation of Modern Man. Gütersloh: Bertelsmann Foundation Publishers. 
Berman, M. (1982). All That Is Solid Melts Into Air: The Experience of Modernity. New York, NY: Simon and Schuster.

Bhaskar, R. (2008). A Realist Theory of Science. London: Verso.

Bhaskar, R., Danermark, B. (2006). 'Metatheory, Interdisciplinarity and Disability Research: A Critical Realist Perspective.' Scandinavian Journal of Disability Research 8(4): 278-290.

Bilimoria, P. (2012). 'Why Is There Nothing Rather Than Something? An Essay in the Comparative Metaphysic of Nonbeing.' Sophia 51: 509-530.

Blau, P.M. (1960). 'Structural Effects.' American Sociological Review 25(2): 178-193.

Blok, A., Elgaard, J.T. (2011). Bruno Latour: Hybrid Thoughts in a Hybrid World. Abingdon: Routledge.

Bottero, W. (2009). 'Relationality and Social Interaction.' The British Journal of Sociology 60(2): 399-420.

Boudon, R. (1981). The Logic of Social Action: An Introduction to Sociological Analysis. London: Francis \& Taylor.

Boudon, R. (1989). Effets perverse et ordre social. Paris: Puf.

Brubaker, R., Cooper, F. (2000). 'Beyond "Identity".' Theory and Society 29(1): 1-47.

Bruni, L., Zamagni, S. (2004). Economia Civile. Efficienza, equità, felicità pubblica. Bologna: il Mulino.

Buber, M. (1970). I and Thou. New York, NY: Scribner.

Burkitt, I. (2016). 'Relational Agency: Relational Sociology, Agency and Interaction.' European Journal of Social Theory 19(3): 322-339.

Burt, R.S. (1992). Structural Holes: The Social Structure of Competition. Cambridge, MA: Harvard University Press.

Caetano, A. (2015). 'Defining Personal Reflexivity: A Critical Reading of Archer's Approach.' European Journal of Social Theory 18(1): 60-75.

Caillé, A. (1993). La démission des clercs. La crise des sciences sociales et l'oubli du politique. Paris: La Découverte.

Calcaterra, V. (2017). 'Relational Social Work at the Case Level. Working With Coping Networks to Cope Micro-Social Problems.' Relational Social Work 1(1): 39-60.

Cappuccio, M., Peeters, A., McDonald, W. (2019). 'Sympathy for Dolores: Moral Consideration for Robots Based on Virtue and Recognition.' Philosophy and Technology 34(1): 129-136.

Clam, J. (2000). 'System's Sole Constituent, the Operation: Clarifying a Central Concept of Luhmannian Theory.' Acta Sociologica 43(1): 63-79.

Coleman, J.S. (1990). Foundations of Social Theory. Cambridge, MA: The Belknap Press of Harvard University Press.

Coleman, J.S. (1993). 'The Rational Reconstruction of Society.' American Sociological Review 58: 1-15.

Collier, A. (1999). Being and Worth. London: Routledge.

Cook, G. and Muir, R. (eds) (2012). The Relational State. How Recognizing the Importance of Human Relationships Could Revolutionise the Role of the State. London: IPPR.

Cross, S., Morris, M. (2003). 'Getting to Know You: The Relational Self-Construal, Relational Cognition, and Well-Being.' Personality and Social Psychology Bulletin 29(4): 512-523.

Crossley, N. (2011). Towards Relational Sociology. Abingdon: Routledge. 


\section{References}

Dahl, R.A. (1979). 'Procedural Democracy.' In: P. Laslett and J. Fishkin (eds). Philosophy, Politics and Society. New Haven, CT: Yale University Press: 97-133.

Dahrendorf, R. (1994). 'The Changing Quality of Citizenship.' In: B. van Steenberger (ed). The Condition of Citizenship. London: Sage: 10-19.

Danermark, B., Ekstrom, M., Jakobsen, L., Karlsson, J. (2002). Explaining Society. Critical Realism in the Social Sciences. London: Routledge.

Davis, K. (1959). 'The Myth of Functional Analysis as a Special Method in Sociology and Anthropology.' American Sociological Review 24(6): 757-772.

Dépelteau, F. (2013). 'Review: Structure and Agency Again, and Much More.' Contemporary Sociology 42(6): 815-819.

Dépelteau, F. (2018). 'From the Concept of 'Trans-Action' to a Process-Relational Sociology.' In: F. Dépelteau (ed). The Palgrave Handbook of Relational Sociology. New York, NY: Palgrave: 499-519.

Dépelteau, F. and Powell, C. (eds) (2013). Applying Relational Sociology. Relations, Networks, and Society. New York, NY: Palgrave.

Derrida, J. (1982). 'Différance.' In: J. Derrida. The Margins of Philosophy. Brighton: Harvester: 1-28.

Destefanis, S., Maietta, O.W. (2015). 'Property Rights and Efficiency in the Care Sector. Evidence from Italy.' JEOD: Journal of Entrepreneurial and Organisational Diversity 4(2): 98-115.

Diwan, R. (2000). 'Relational Wealth and the Quality of Life.' Journal of SocioEconomics 29(4): 305-340.

Donati, P. (2001). Il lavoro che emerge. Prospettive del lavoro come relazione sociale in una economia dopo-moderna. Turin: Bollati Boringhieri.

Donati, P. (2009). 'Beyond the Dilemmas of Multiculturalism: Recognition Through 'relational reason'.' International Review of Sociology 19(1): 55-82.

Donati, P. (2010). La matrice teologica della società. Soveria Mannelli: Rubbettino.

Donati, P. (2011a). Relational Sociology. A New Paradigm for the Social Sciences. London: Routledge.

Donati, P. (2011b). 'Modernisation and Relational Reflexivity.' International Review of Sociology 21(1): 21-39.

Donati, P. (2015a). 'Manifesto for a Critical Realist Relational Sociology.' International Review of Sociology 25(1): 86-109.

Donati, P. (2015b). Social Mechanisms and Their Feedbacks: Mechanical vs Relational Emergence of New Social Formations. In: M.S. Archer (ed). Generative Mechanisms Transforming the Social Order. New York, NY: Springer: 65-92.

Donati, P. (2015c). L'enigma della relazione. Milano-Udine: Mimesis edizioni.

Donati, P. (2019). 'Discovering the Relational Goods: Their Nature, Genesis and Effects.' International Review of Sociology 29(2): 238-259.

Donati, P. (2020). 'Impact of AI/Robotics on Human Relations: Co-Evolution Through Hybridisation.' In: J. von Braun et al. (eds). Robotics, AI and Humanity; Science, Ethics, and policy. Dordrecht: Springer.

Donati, P. (2021). 'Being Human (or What?) in the Digital Matrix Land: The Construction of the Humanted.' In: M. Carrigan and D. Porpora (eds). PostHuman Futures. Human Enhancement, Artificial Intelligence and Social Theory. London: Routledge.

Donati, P., Archer, M.S. (2015). The Relational Subject. Cambridge: CUP.

Donati, P. and Colozzi, I. (eds) (2001). Generare "il civile": nuove esperienze nella società italiana. Bologna: il Mulino. 
Donati, P., Solci, R. (2011). I beni relazionali. Che cosa sono e quali effetti producono. Turin: Bollati Boringhieri.

Douglas, M. (1986). How Institutions Think. Syracuse, NY: Syracuse University Press.

Durkheim, E. (1951 [1897]). Suicide: A Study in Sociology. New York, NY: Free Press.

Eacott, S. (2018). 'Embedded and Embodied Auctors.' In: Beyond Leadership, A Relational Approach to Organisational Theory in Education. New York, NY: Springer: 81-94.

Elder-Vass, D. (2009). Towards a Social Ontology of Market System. University of Essex, CRESI Working Paper Number 2009-06.

Elder-Vass, D. (2018). 'Lifeworld and Systems in the Digital Economy.' European Journal of Sociology 21(2): 227-244.

Elias, N. (1978). What Is Sociology?. New York, NY: Columbia University Press.

Elster, J. (2017). 'The Temporal Dimension of Reflexivity: Linking Reflexive Orientations to the Stock of Knowledge.' Distinktion 18(3): 274-293.

Emirbayer, M. (1997). 'Manifesto for a Relational Sociology.' American Journal of Sociology 103(2): 281-317.

Engels, F. (1959). Anti-Dühring. Moscow: Progress Publisher.

Evers, A. (2015). 'Social Innovations on the Local Level. Approaches, Instruments and Different Ways of Dealing With Them.' In: P. Donati and L. Martignani (eds). Towards a New Local Welfare. Best Practices and Networks of Social Inclusion. Bologna: Bononia University Press.

Fiske, A., Haslam, N. (1996). 'Social Cognition Is Thinking About Relationships.' Current Directions in Psychological Science 5(5): 143-148.

Fleming, L., Mingo, S., Chen, D. (2007). 'Collaborative Brokerage, Generative Creativity, and Creative Success.' Administrative Science Quarterly 52(3): 443-475.

Floridi, L. (ed) (2015). The Onlife Manifesto: Being Human In a Hyperconnected Era. New York, NY: Springer.

Fløysand, A., Jakobsen, S.E. (2011). 'The Complexity of Innovation: A Relational Turn.' Progress in Human Geography 35(3): 328-344.

Folgheraiter, F. (2004). Relational Social Work. Toward Networking and Societal Practices. London: J. Kingsley.

Folgheraiter, F., Raineri, M.L. (2012). 'A Critical AnaFoucault, M. (1966). Les mots et les choses. Paris: Gallimard.

Frankfurt, H.G. (1988). The Importance of What We Care About. Cambridge: CUP.

Gambino, G. (ed) (2017). Patologie dell'identità e ipotesi di terapia filosofica. Rome: Edizioni Jus Quia Justum.

Gelfand, M.J. et al. (2006). 'Negotiating Relationally: The Dynamics of the Relational Self in Negotiations.' The Academy of Management Review 31(2): 427-451.

Genovesi, A. (2005). Lezioni di commercio o sia di economia civile [1765-1767]. Napoli: Istituto Italiano per gli studi filosofici.

Gergen, K.J. (1973). 'Social Psychology as History.' Journal of Personality and Social Psychology 26: 309-320.

Gergen, K.J. (1994). Realities and Relationships: Soundings in Social Constructionism. Cambridge, MA: Harvard University Press.

Gergen, K.J. (2009). Relational Being: Beyond Self and Community. Oxford: Oxford University Press. 
Gergen, K.J. (2015a). 'From Mirroring to World-Making: Research as Future Forming.' Journal for the Theory of Social Behaviour 45(3): 287-310.

Gergen, K.J. (2015b). 'Toward a Relational Humanism.' Journal of Humanistic Counselilysis of the Social Work Definition According to the Relational Paradigm.' International Social Work 55(4): 473-487.

ng 54: 149-165.

Giddens, A. (1992). The Transformation of Intimacy. Sexuality, Love and Eroticism in Modern Societies. Cambridge: Polity Press.

Giddens, A. (1998). The Third Way. The Renewal of Social Democracy. Cambridge: Polity Press.

Goldsmith, S., Georges, G., Burke, T.G. (2010). The Power of Social Innovation. New York, NY: Wiley.

González, A.M. (2011). 'Kant's Philosophy of Education: Between Relational and Systemic Approaches.' Journal of Philosophy of Education 45(3): 433-454.

González, A.M. (2017). 'In Search of a Sociological Explanation for the Emotional Turn.' Sociologia. Problemas y Prácticas 85: 27-45.

Gray, J. (1993). Post-Liberalism. Studies in Political Thought. London: Routledge.

Greenfield, S. (2009). ID: The Quest for Identity in the 21st Century. London: Sceptre.

Greenfield, S. (2014). Mind Change: How Digital Technologies are Leaving Their Mark on Our Brains. New York, NY: Random House.

Gulati, R. (2007). Managing Network Resources: Alliances, Affiliation, and Other Relational Assets. Oxford: Oxford University Press.

Günther, G. (1976). 'Formal Logic, Totality and the Super-Additive Principle.' In: Beiträge zur Grundlegung einer operationsfähigen Dialektik. Hamburg: Felix Meiner: 329-351.

Guy, J.S. (2018). 'Niklas Luhmann before Relational Sociology: The Cybernetics Roots of Systems Theory.' Systems Research and Behavioural Science 35: $856-868$.

Habermas, J. (1984). Theory of Communicative Action. Volume One: Reason and the Rationalization of Society. Boston, MA: Beacon Press.

Habermas, J. (1987). Theory of Communicative Action. Volume Two: Lifeworld and System: A Critique of Functionalist Reason. Boston, MA: Beacon Press.

Habermas, J. (1998). The Inclusion of the Other: Studies in Political Theory. Cambridge: MIT Press.

Hałas, E. (2021). 'Relational Care: Rethinking Altruism.' In: E. Hałas and A. Manterys (eds). Relational Reason, Morals and Sociality. Berlin: Peter Lang: 69-93.

Halsall, F. (2012). 'Niklas Luhmann and the Body: Irritating Social Systems.' The New Bioethics 18(1): 4-20.

Harari, Y.N. (2017). Homo Deus: A Brief History of Tomorrow. New York, NY: Harper Collins.

Harman, G. (2009). Prince of Networks: Bruno Latour and Metaphysics. Melbourne: re.press.

Haslam, N. (2006). 'Dehumanization: An Integrative Review.' Personality and Social Psychology Review 10(3): 252-264.

Haythornthwaite, C. (2002). 'Strong, Weak, and Latent Ties and the Impact of New Media.' The Information Society: An International Journal 18(5): 385-401.

Haythornthwaite, C. (2005). 'Social Networks and Internet Connectivity Effects.' Information, Communication \& Society 8(2): 125-147. 
Heidegger, M. (1969). Identity and Difference. Chicago, IL: University of Chicago Press.

Heidegger, M. (1999). 'Letter on 'Humanism.' In: M. Heidegger. Pathmarks. Cambridge: CUP.

Hirsch, F. (1995). Social Limits to Growth. London: Routledge.

Hong, G.Y. (2004). 'Emotions in Culturally-Constituted Relational Worlds.' Culture \& Psychology 10(1): 53-63.

Horstink, T. (2011). 'The Effects of Social Networks on Group Moral Reasoning in the Royal Netherlands Army.' Universiteit Twente: Communication studies, Master Thesis.

Ignatieff, M. (1984). The Needs of Strangers. London: Chatto and Windus.

Jalava, J. (2003). 'From Norms to Trust: The Luhmannian Connections between Trust and System.' European Journal of Social Theory 6: 173-190.

Jensen, T. (2010). 'Beyond Good and Evil: The Adiaphoric Company.' Journal of Business Ethics 96: 425-434.

Jindra, M., Jindra, I.W. (2015). A Revolutionary Change: Relational Work in the New Safety Net. University of Notre Dame: Dept. of Anthropology.

Junge, M. (2001). 'Zygmunt Bauman's Poisoned Gift of Morality. British Journal of Sociology 52(1): 105-119.

Knudsen, N. (2019). 'Relationality and Commitment: Ethics and Ontology in Heidegger's Aristotle.' Journal of the British Society for Phenomenology 50(4): 337-357.

Korsgaard, C. (2013). 'The Relational Nature of the Good.' In: R. Shafer-Landau (ed). Oxford Studies on Metaethics, vol. 8. Oxford: Oxford University Press.

Laaser, K., Bolton, S. (2017). 'Ethics of Care and Co-Worker Relationships in UK Banks.' New Technology, Work and Employment 32(3): 213-227.

LaGrandeur, K. (2015). 'Emotion, Artificial Intelligence, and Ethics.' In: J. Romport, E. Zackova, and J. Kelemen (eds). Beyond Artificial Intelligence: The Disappearing Human-Machine Divide. Dordrecht: Springer: 97-110.

Lally, E. (2002). At Home with Computers. Oxford/New York: Berg.

Lasch, C. (1979). The Culture of Narcisism. New York, NY: Norton.

Latour, B. (1999). Pandora's Hope: Essays on the Reality of Science Studies. Cambridge, MA: Harvard University Press.

Lazega, E. (2007). Réseaux sociaux et structures relationnelles. Paris: Puf.

Lazega, E., Pattison, P.E. (2001). 'Social Capital as Social Mechanisms and Collective Assets: The Example of Status Auctions Among Colleagues.' In: N. Lin, K. Cook, and R.S. Burt (eds). Social Capital: Theory and Research. New York, NY: Aldine de Gruyter, chapter 8.

Leydesdorff, L. (2014). 'Advances in Science Visualization: Social Networks, Semantic Maps, and Discursive Knowledge.' In: B. Cronin and C. Sugimoto (eds). Beyond Bibliometrics: Harnessing Multidimensional Indicators of Scholarly Impact. Cambridge, MA: MIT Press.

Lidz, V. (1981). 'Conceptions of Value-Relevance and the Theory of Action.' Sociological Inquiry 51(3-4): 371-408.

Lindgren, J., Liukkonen, J. (2019). 'Quantum Mechanics can be Understood through Stochastic Optimization on Spacetimes.' Scientific Reports 9: 1-8. Doi: https://doi.org/10.1038/s41598-019-56357-3.

Lindhard, T. (2019). 'Consciousness from the Outside-In and Inside-Out Perspective.' Journal of Consciousness Explorations \& Research 10(3): 1-15. 
Lockwood, D. (1992). 'Social Integration and System Integration.' In: Solidarity and Schism. 'The Problem of Disorder' in Durkheimian and Marxist Sociology. Oxford: Clarendon Press: 399-412.

Luhmann, N. (1976). 'The Future Cannot Begin: Temporal Structures in Modern Society.' Social Research 43: 130-152.

Luhmann, N. (1990). 'Sthenography.' Stanford Literature Review 7: 133-137.

Luhmann, N. (1993). 'The Code of the Moral.' Cardozo Law Review 14(3-4): 995-1009.

Luhmann, N. (1995). Social Systems. Palo Alto, CA: Stanford University Press.

Luhmann, N. (1996). 'The Sociology of the Moral and Ethics.' International Sociology 11(1): 27-36.

Luhmann, N. (1997). 'Limits of Steering.' Theory, Culture and Society 14(1): 41-57.

Luhmann, N. (1998). Observations on Modernity. Stanford, CA: Stanford University Press.

Luhmann, N. (2008a). Die Moral der Gesellschaft. Frankfurt a.M.: Suhrkamp.

Luhmann, N. (2008b). 'Are There Still Indispensable Norms in Our Society?.' Soziale Systeme 14(1): 18-37.

Luhmann, N. (2002). Theories of Distinction: Redescribing the Descriptions of Modernity. Stanford, CA: Stanford University Press.

Lyotard, J.F. (1984). The Postmodern Condition: A Report on Knowledge. Minneapolis, MN: University of Minnesota Press.

Mahoney, A. (2010). 'Religion in Families, 1999-2009: A Relational Spirituality Framework,' Journal of Marriage and Family 72: 805-827.

Malabou, C. (2009). Ontologie de l'accident: Essai sur la plasticité destructrice. Paris: Léo Scheer.

Malfer, L. (ed) (2019). New Public Family Management. Milan: FrancoAngeli.

Manterys, A. (2017). 'Relational Sociology Paradigms.' Stan Rzeczy [State of Affairs] 1(12): 67-94.

Marques, E. (2012). 'Social Networks Matter in Gaining Access to Goods and Services Obtained from Outside Markets.' International Journal of Sociology 41: $10-27$.

Maxwell, L. (2012). 'Toward an Agonistic Understanding of Law: Law and Politics in Hannah Arendt's Eichmann in Jerusalem.' Contemporary Political Theory 11(1): 88-108.

Mendoza, X., Vernis, A. (2008). 'The Changing Role of Governments and the Emergence of the Relational State.' Corporate Governance 7(4): 389-396.

Merton, R.K. (1938). 'Social Structure and Anomie.' American Sociological Review 3(5): 672-682.

Merton, R.K. (1968). Social Theory and Social Structure. New York, NY: The Free Press.

Metcalfe, A., Game, A. (2012). 'In the Beginning is Relation': Martin Buber's Alternative to Binary Oppositions.' Sophia 51(3): 351-363.

Moreh, J. (1986). 'Morality and Welfare.' Theory and Decision 21: 209-230.

Mulgan, G. (2012). 'Government with the People: The Outlines of a Relational State.' In: G. Cook and R. Muir (eds). The Relational State. How Recognizing the Importance of Human Relationships Could Revolutionise the Role of the State. London: IPPR.

Nørskov, M. (2015). 'Revisiting Ihde's Fourfold “Technological Relationships”: Application and Modification.' Philosophy \& Technology 28(2): 189-207. 
Overton, W.F. (2014). 'The Process-Relational Paradigm and RelationalDevelopmental-Systems Metamodel as Context.' Research in Human Development, 11: $323-331$.

Pana, L. (2006). 'Artificial Intelligence and Moral Intelligence.' TripleC 4(2): 254-264.

Pitts-Taylor, V. (2016). The Brain's Body: Neuroscience and Corporeal Politics. Durham: Duke University Press.

Papilloud, C. (2018) 'Georg Simmel and Relational Sociology.' In: F. Dépelteau (ed). The Palgrave Handbook of Relational Sociology. New York, NY: Palgrave: 212-273.

Parsons, T. (1967). 'Christianity and Modern Industrial Society.' In: Sociological Theory and Modern Society. New York, NY: Free Press.

Parsons, T. (1977). Social Systems and the Evolution of Action Theory. New York, NY: Free Press.

Parsons, T. (2007). American Society. A Theory of the Societal Community. BoulderLondon: Paradigm Publ.

Pestoff, V. (2009). A Democratic Architecture for the Welfare State. London: Routledge.

Pestoff, V. (2015). 'Co-Production as Social Innovation in Public Services.' In: P. Donati and L. Martignani (eds). Towards a New Local Welfare. Best Practices and Networks of Social Inclusion. Bologna: Bononia University Press.

Porpora, D. (2018a). 'Critical Realism as Relational Sociology.' In: F. Dépelteau (ed). The Palgrave Handbook of Relational Sociology. New York, NY: Palgrave Macmillan: 413-430.

Porpora, D. (2018b). 'Materialism, Emergentism, and Social Structure: A Response to Wendt's Quantum Mind.' Journal for the Theory of Social Behaviour 48(2): 183-187.

Powell, C. and Dépelteau, F. (eds) (2013). Conceptualizing Relational Sociology. Ontological and Theoretical Issues. New York, NY: Palgrave.

Prodi, R. (2002). 'La mia visione dell'Europa.' In: C. D’Adda (ed). Per l'economia italiana. Scritti in onore di Nino Andreatta. Bologna: il Mulino.

Rasch, W. (2012). 'Luhmann's Ontology.' Revue Internationale de Philosophie 259(1): 85-104.

Reader, S. (2007). 'The Other Side of Agency.' Philosophy 82: 579-604.

Ricoeur, P. (1954). 'Sympathie et Respect: Phénoménologie et Éthique de la seconde personne.' Revue de Métaphysique et de Morale 59(4): 380-397.

Ricoeur, P. (1990). Soi-même comme un autre. Paris: Seuil.

Ricoeur, P. (2004). Parcours de la reconnaisance. Paris: Éditions Stock.

Rorty, R. (1999). Philosophy and Social Hope. London: Penguin Books.

Rovelli, C. (2020). Helgoland. Milan: Adelphi.

Rushworth, J., Schluter, M. (2011). Transforming Capitalism from Within. A Relational Approach to the Purpose, Performance, and Assessment of Companies. Cambridge: Relationships Global Report No. 1.

Scheve (von), C. (2018). 'A Social Relational Account of Affect.' European Journal of Social Theory 21(1): 39-59.

Seikkula, J., Arnkil, T.M. (2006). Dialogical Meet Social Networks. London: Karnac Books.

Seligman, A. (1992). The Idea of Civil Society. New York, NY: Free Press. 
Shaikh, A. (1990). 'Capital as a Social Relation.' In: John Eatwell et al. (eds). The New Palgrave: A Dictionary of Economic Theory and Doctrine. London: Macmillan Press: $72-78$.

Shih, C. (2020). 'Introduction.' In: Chih-yu Shih et al. China and International Theory. The Balance of Relationships. London: Routledge.

Shotter, J. (2012). 'Gergen, Confluence, and his Turbulent, Relational Ontology: The Constitution of Our Forms of Life within Ceaseless, Unrepeatable, Intermingling Movements.' Psychological Studies 57(2): 134-141.

Simmel, G. (1970). Saggi di estetica. Padua: Liviana.

Simmel, G. (1989). Philosophie des Geldes [1900]. Frankfurt a.M.: Suhrkamp (English tr. Philosophy of Money. London: Routledge, 2004).

Soros, G. (2000), 'Reflexivity in Financial Markets.' In: Open Society. Reforming Global Capitalism. New York, NY: Public Affairs: 58-90.

Spencer-Brown, G. (1979). Laws of Form. New York, NY: Dutton.

Stanford Encyclopedia of Philosophy (2018). Supervenience. Stanford, CA: Stanford University.

Stein, E. (1989). On the Problem of Empathy. Washington, DC: ICS Publications.

Stets, J.E., Carter, M.J. (2012). 'A Theory of the Self for the Sociology of Morality.' American Sociological Review 77(1): 120-140.

Stichweh, R. (2000). 'System Theory as an Alternative to Action Theory? The Rise of 'Communication' as a Theoretical Option.' Acta Sociologica 43(1): 5-13.

Stiglitz Report (2009). Report by the Commission on the Measurement of Economic Performance and Social Progress, by Joseph Stiglitz, Amartya Sen, Jean-Paul Fitoussi (www.stiglitz-sen-fitoussi.fr).

Streeck, W. (2012). 'How to Study Contemporary Capitalism?' European Journal of Sociology 53(1): 1-28.

Tam, T. (1989). 'Demarcating the Boundaries Between Self and the Social: The Anatomy of Centrality in Social Networks.' Social Networks 11(4): 387-401.

Teubner, G. (2002). 'Hybrid Laws: Constitutionalizing Private Governance Networks.' In: R. Kagan and K. Winston (eds). Legality and Community. Berkley, CA: Berkeley Public Policy Press: 311-331.

Teubner, G. (2006a). 'The Anonymous Matrix: Human Rights Violations by 'Private' Transnational Actors.' Modern Law Review 69(3): 327-346.

Teubner, G. (2006b). 'Rights of Non-Humans? Electronic Agents and Animals as New Actors in Politics and Law.' Journal of Law and Society 33(4): 497-521.

Teubner, G. (2009). 'Self-Subversive Justice: Contingency or Transcendence Formula of Law.' The Modern Law Review 72: 1-23.

Teubner, G. (2011). 'A Constitutional Moment. The Logics of "Hitting the Bottom.' In: P. Kjaer, G. Teubner, and A. Febbrajo (eds). The Financial Crisis in Constitutional Perspective: The Dark Side of Functional Differentiation. Oxford and Portland: Hart: 3-42.

Teubner, G. (2012). Verfassungsfragmente: Gesellschaftlicher Konstitutionalismus in der Globalisierung. Berlin: Suhrkamp.

Uhlaner, C.J. (2014). 'Relational Goods and Resolving the Paradox of Political Participation.' Recerca. Journal of Thought and Analysis 14: 47-72.

Vandenberghe, F. (2002). 'Reconstructing Humants: A Humanist Critique of Actant-Network Theory.' Theory, Culture \& Society 19(5/6): 51-67.

Vandenberghe, F. (2016). 'The Relation as Magical Operator: Overcoming the Divide Between Relational and Processual Sociology.' In F. Dépelteau (ed). The Palgrave Handbook of Relational Sociology. New York, NY: Palgrave: 35-57. 
Van de Walle, G. (2011). 'Becoming Familiar With a World': A Relational View of Socialization.' International Review of Sociology 21(2): 315-333.

Vidal, I. (2015). 'The Role of Third Sector in Local Welfare.' In: P. Donati and L. Martignani (eds). Towards a New Local Welfare. Best Practices and Networks of Social Inclusion. Bologna: Bononia University Press.

Wang, P., Robins, G., Pattison, P., Lazega, E. (2015). 'Social Selection Models for Multilevel Networks.' Social Networks. Doi: 10.1016/j.socnet.2014.12.003.

Weaver, B. (2016). Offending and Desistance. The Importance of Social Relations. Abingdon: Routledge.

Weaver, B., McNeill, F. (2015). 'Lifelines: Desistance, Social Relations, and Reciprocity.' Criminal Justice and Behaviour 42(1): 95-107.

Weber, M. (2013 [1922]). Economy and Society. Berkeley-Los Angeles: University of California Press, vol. 1.

Wendt, A. (2015). Quantum Mind and Social Science: Unifying Physical and Social Ontology. Cambridge: CUP.

White, H.C. (1992). Identity and Control: A Structural Theory of Social Action. Princeton, NJ: Princeton University Press.

White, H.C. (2008). Identity and Control: How Social Formations Emerge. Princeton, NJ: Princeton University Press.

White, S.C. (2017). 'Relational Wellbeing: Re-Centring the Politics of Happiness, Policy and the Self.' Policy \& Politics 45(2): 121-136.

Whittle, A., Spicer, A. (2008). 'Is Actor Network Theory Critique?.' Organisation Studies 29(4): 611-629.

Zelizer, V. A. (2012). 'How I Became a Relational Economic Sociologist and What Does That Mean?.' Politics \& Society 40(2): 145-174.

Zubero, I. (2018). 'El Tercer Sector como movimiento voluntariadista: una propuesta para repensar la identidad del TSAS desde el paradigma de la democracia del cuidado.' Revista Española del Tercer Sector 38(1): 43-68. 


\section{Name Index}

Note: Page numbers followed by "n" refer to notes.

Adams, M. 197

Ahuja, G. 160

Alexander, J.C. 23, 24

Andersen, T. 79n2

Anders, G. 217, 227

Aneesh, A. 211

Archer, M.S. 6, 11, 55, 57, 60, 63-65, 79n5, 104n9, 126, 151, 153, 154, 169-171, 182, 186, 203n5, 214

Arnkil, T.M. 79

Aspers, P. 179

Avnon, D. 175

Azarian, R. 175

Baker, L.R. 171, 199, 200, 203, 214-215

Bateson, G. 55

Baudrillard, J. 220

Bauman, Z. 57, 119n1, 131n2

Bauwens, M. 105

Beckert, J. 24

Beck-Gernsheim, E. 25

Beck, U. 2, 4, 22, 25, 88, 124, 151, 152

Bekoff, M. 200

Beniger, J. 2

Berger, P.L. 72, 83n7

Berman, M. 73

Bhaskar, R. 52, 53

Bilimoria, P. 215

Blau, P.M. 95, 138

Blok, A. 62

Bolton, S. 208

Bonss, W. 88, 124, 151, 152

Bottero, W. 91, 92

Boudon, R. 18, 132

Brubaker, R. 189

Bruni, L. 105, 145

Buber, M. 101, 175
Burkitt, I. 173

Burt, R.S. 160

Caetano, A. 173

Caillé, A. 34

Calcaterra, V. 158

Cappuccio, M. 198

Carter, M.J. 174

Castells, M. 2

Clam, J. 207

Coleman, J.S. 110, 136

Collier, A. 100

Colozzi, I. 102

Cook, G. 47

Cooper, F. 189

Cross, S. 201

Dahl, R.A. 21

Dahrendorf, R. 36

Danermark, B. 52, 53

Davis, K. 40

Dépelteau, F. 4, 54, 55, 64, 186

Derrida, J. 220

Destefanis, S. 41

Diwan, R. 40

Donati, P. 11, 27n2, 29, 31, 42, 45-48, 57, $59,64,66,72,79,86,87,94,102,106$, $110,124,139,143,175,180 \mathrm{n} 2,182,184$, 189, 193, 203n6, 205n7, 215, 218-219

Donzelot, J. 22

Douglas, M. 112, 130

Durkheim, E. 3, 6, 29, 30, 61, 72, 82, $112,127,218,229$

Eacott, S. 205n7

Elder-Vass, D. 132, 208

Elgaard, J.T. 62 
Elias, N. 22, 77

Elster, J. 203

Emirbayer, M. 6, 53, 54, 179, 186

Engels, F. 219

Esposito, M. 143

Etzioni, A. 2

Evers, A. 123

Fiske, A. 173

Fleming, L. 160

Floridi, L. 26, 193n1, 207, 213

Fløysand, A. 123

Folgheraiter, F. 158

Foucault, M. 22, 155, 220

Fuhse, J. 175

Gambino, G. 174

Game, A. 175

Gelfand, M.J. 169

Genovesi, A. 145

Georges, G. 102

Gergen, K.J. 52-54

Giddens, A. 2, 3, 24, 108, 114, 121, 165

Glynn Burke, T. 102

Goldsmith, S. 102

González, A.M. 163-164, 173

Gray, J. 16

Greenfield, S. 197, 199

Gulati, R. 160

Günther, G. 91n8, 110

Guy, J.S. 223

Habermas, J. 24, 36, 101, 208

Hałas, E., 127

Halsall, F. 214

Harari, Y.N. 193n1

Harman, G. 62

Haslam, N. 173, 212

Haythornthwaite, C. 120

Heidegger, M. 179, 180, 180n3, 215

Hirsch, F. 20

Hong, G.Y. 173

Horstink, T. 119

Ignatieff, M. 145

Jakobsen, S.E. 123

Jalava, J. 113

Jensen, T. $131 \mathrm{n} 2$

Jindra, I.W. 158

Jindra, M. 158

Kanter, R.M. 143

Knudsen, N. 179
Kohl, S. 179

Korsgaard, C. 163, 163n1

Laaser, K. 208

LaGrandeur, K. 202

Lally, E. 202

Lasch, C. 151

Lash, S. 2

Latour, B. 25, 62, 177, 216, 216n1, 217

Lau, C. 88, 124, 151, 152

Lazega, E. 160

Leydesdorff, L. 123

Lidz, V. 165

Lindgren, J. 57

Lindhard, T. 198

Liukkonen, J. 57

Lockwood, D. 23n1

Luckmann, T. 72, 83n7

Luhmann, N. 4-6, 8, 25, 33, 35, 36, 52, $54,58,72,87,103,112,113,126,128$, 200, 202n4, 207, 214, 216, 220, 223, 224

Lyotard, J.F. 6, 220

Mahoney, A. 224

Maietta, O.W. 41

Malabou, C. 197

Malfer, L. 47

Manterys, A. 42

Marques, E. 158

Marx, K. 2, 3

Maxwell, L. 155

McClelland, D. 2

McNeill, F. 158

Mendoza, X. 49

Merton, R.K. 116, 141

Metcalfe, A. 175

Moreh, J. 125-126

Morris, M. 201

Muir, R. 47

Mulgan, G. 47-48

Nietzsche, F.W. 211

Nørskov, M. 198

Overton, W.F. 178

Pana, L. 200

Papilloud, C. 3, 4

Parsons, T. 16, 23-24, 33, 87, 113, 159

Pattison, P. 160

Pestoff, V. 12

Pierce, J., 200

Pitts-Taylor, V. 204 
Porpora, D. 52, 57n2, 197n2

Porter, M. 143

Powell, C. 4, 54, 186

Prodi, R. 113-114

Proust, M. 1

Raineri, M.L. 158

Rasch, W. 52

Reader, S. 127

Ricoeur, P. 57, 168, 171, 181

Robins, G. 160

Rorty, R. 52, 221

Rovelli, C. 221

Rushworth, J. 89, 103, 105

Scheve (von), C. 62, 173

Schluter, M. 89, 103, 105

Seikkula, J. 79

Seligman, A. 20, 23

Shaikh, A. 93

Shih, C. 98

Shotter, J. 53, 207

Simmel, G. 3-4, 3-5, 72, 80n6, 87, 110,212

Solci, R. 106

Soros, G. 39, 133

Spencer-Brown, G. 202n4

Spicer, A. 62

Stein, E. 171
Stets, J.E. 174

Stichweh, R. 203

Streeck, W. 93

Tam, T. 77, 175

Tawney, R. 2

Teubner, G. 26, 112, 124, 132n4, 154, 206, 207, 224n4

Titmuss, R. 159

Uhlaner, C.J. 185

Vandenberghe, F. 55, 216

Van de Walle, G. 173

Vernis, A. 49

Vidal, I. 123

Wang, P. 160

Weaver, B. 50, 64, 96, 158

Weber, M. 3, 29, 61, 65, 72, 86, 127, 211, 218, 223

Wendt, A. 197n2

White, H.C. 55, 56n1, 174, 175

White, S.C. 173

Whittle, A. 62

Zamagni, S. 105, 145

Zelizer, V.A. 77

Zubero, I. 41 


\section{Subject Index}

Note: Page numbers in bold refer to tables.

Achieving Society, The (McClelland) 2
Acquisitive Society, The (Tawney) 2
Active Society, The (Etzioni) 2
actor network theory (ANT) 25, 62, 188
adaptive morphogenesis 207
addictive society 154
aftermodernity (aftermodern) 2, 8, 9, 17,
40, 42-46, 88-90, 94, 101, 110, 124, 230;
features of 45-46; relational approach
to freedom vs control dilemmas 27-33
agency-structure relationship 6, 7, 111-112
AGIL diagram 23, 27n2, 30, 31, 124,
125, 134, 136
AI see artificial intelligence (AI) algocracy (the rule of algorithms) 211

Alter 21, 55, 56, 67, 74, 78, 80, 98, 117-119, $127,131,142,160,171,175,177,184,185$ Alternative Investment Market 42 altruism 159

ANT see actor network theory (ANT) Arab Spring 113

artificial intelligence (AI) 35, 94, 188, 193, 194, 208-210, 217

auctor $205 \mathrm{n} 7$

autonomous reflexivity $64,122,124$, 143,183

body-mind relationship 196, 200

Bolsa de Valores Sociales y Ambietais (BVS\&A) 42

bound morphogenesis 124

bureaucratisation of society 6

BVS\&A see Bolsa de Valores Sociales y Ambietais (BVS\&A)

capital 46, 101; financial capital 103; human capital 95; Marxian concept of 6, 106; social capital 6, 51, 95-97, 100, 105-107, 118, 160, 161

capitalism 36, 151, 153; casino 19;

direction of 90-95; evolution of 51; of social networks 95

capitalist relation 92,93

Capitalist Society (Marx) 2

capitalist-worker relationship 95

causality 117, 118, 130, 131, 142; linear

10, 135; prevailing explanations of, questioning 133-136; social 145

citizenship $38,154,177$; corporate 46 ; definition of 36 ; right of 121, 153; social 135; societal 33

civil economy $6,25,41,95,105,144$, 145,153

civil society $23,26,31,33,37,38,42-47$, 49, 50, 101, 103, 118, 119, 121, 146, 149, $153,177,178$

collective addiction 154

collective conscience $18,82,112,127$, 166

collective identity, in morphogenetic process $186-188$

collective morality 111, 153, 165

common good $36,44,108,125,139$,

150-152, 157, 161, 163, 165, 228

communicative action 101

communicative reflexivity $64,122,124$, 183

concomitant compatibility $104 \mathrm{n} 9$

concomitant complementarity 85, 104, 104n9

conformist reflexivity 201

conservation vs progress 7

constraining contradiction 104n9

constructivism 51, 53, 222; 
contingent complementarity 104 , 104n9, 197

Copernican revolution 89

corporate social responsibility (CSR) 143

Covid-19 pandemic 1, 3, 7, 8, 11-12, 35, 98

critical realism 52-53, 58, 199

critical reflexivity 202

CSR see corporate social responsibility (CSR)

cultural identity 173, 187, 195, 196

cultural nihilism 216

Darwinian evolution 38-39

deconstructionism 52

dematerialisation 1, 2, 196, 197

Digital Technological Matrix (DTM)

193-195, 197, 201, 202, 206, 207, 210

division of social labour 6, 30

doctor-patient relation 78

domains, definition of $56 \mathrm{n} 1$

double bind 114, 124, 127, 155

double effect 131

DTM see Digital Technological Matrix (DTM)

ecological fallacy (or ecological inference fallacy) $174,174 \mathrm{n} 1$

Ego 21, 55, 56, 63, 67, 74, 75, 77, 78, 80, 98, 117, 118, 119, 127, 131, 160, 168, $169,171,172,175,177,178,180,184$, $185,210,219$

emergence (emergent) 5, 8, 21, 31, 33, $35,36,42,56-58,60,61,63.65,74$, 75, 77, 80-82, 85, 88, 90-92, 95, 98, $102,108,112,118,123,127,128,133$, 137-139, 141, 145, 155, 159, 202, 217, 221, 223-225; of hybridised institutions and organisations 205-207; in morphogenetic processes, theory of 4; of new social subjects generating relational goods 167-189; relational effects 9; of subjectivity, morality as 128 ; systemic, morality as 127 ; theory of 4

employer-employee relationship 78

enigma of the relation 218-220, 228

equality $16,18,22,32,83,113,114,153$, 180

ethical good 139

eudemonic morality 161-163

eurialistics 220
European Union 113, 122n2

exchange value 99-101

family group conference (FGC) 79, $79 \mathrm{n} 3$

family relation $85,92,171,177$

feedback: negative $79,116-118$; positive 79, 80, 91, 116-118; relational 6, 79, $80,91,117,125,180,184,186$

feudal relation 92

FGC see family group conference (FGC)

financial capital 103

founding relation $91 \mathrm{n} 8,119,180$

freedom vs control, lib/lab paradigm of 15-34; aftermodern society 27-33; conducted within modernity, attempts to reconcile 23-25; limitations 25-27; modernity $15-17$; obsolescence 25-27; paradoxes and empirical reality 19-22

freedom vs equality $15-16$

free market laws 132

friendship relation $78,92,160-161$

functionalism 40; competitive skills of 40; positivistic 17-18; structural neo-functionalism 25, 38; systemic 151

functionalist theory of social systems 136

gazing vs observing 58-59

GDP see gross-domestic product (GDP)

GDWB see Gross Domestic Well-Being (GDWB)

Gemeinschaft 17, 72

generative mechanism 9, 47, 52, 59, 71, $79-82,117,175,184$

generative semantics of the relation 75 , 81

Gesellschaft 72

GEXSI (Global Exchange For Social Investment) 42

good life 10-11, 63, 149-166; definition of 149; eudemonic morality 161-163; human happiness, classical and modern conceptions of 149-153; logic of opportunity, globalisation and 154-155; morality of social relations 158-163; moral opportunities 153-154; relational character of the moral good 163-164; relational logic of happiness 155-157; through social relations $164-166$ 
gross-domestic product (GDP) 40, 41 Gross Domestic Well-Being (GDWB) $40,41,45,50$ group consciousness 115

habitus 79, 91, 150, 177

happiness paradox 40

harm 8, 132-138, 141-144, 146, 172, 207, 208

hetero-control vs self-control 29

HHI see human-human interaction (HHI)

Hobbesian solution 206-207

holism 5, 17, 18, 58, 136, 161

Homo faber 229

HRI see human-robot interactions (HRI)

human capital 95

human happiness, classical and modern conceptions of 149-153

human-human interaction (HHI) 198

human/non-human distinction 218-220

human qualities and properties 211-212

human-robot interactions (HRI) 198

human transcendence 212, 216

hybridisation processes 195, 197-202, 204, 207, 210

hybridised society 193-210; identities and relations 199-204; institutions and organisations, emergence of 205-207; issues 194-199; rationale for 193-194; scenarios 207-209

hyper-modernity (hyper-modern) 2, 10, 229

ICTs see information and communication technologies (ICTs)

identity $167-175$; cultural identity 173, 187, 195, 196; hybridisation of 199-204; idem identity (the selfsame) $57,168,180 \mathrm{n} 3,181$; individual identity 169, 172, 182; ipse identity (the self) $57,168,180 \mathrm{n} 3,181$; personal identity $64,129,167,169,171,174,175$, $178,179,186,188,189,225$; recognition of 181-182; relational identity $169,171-173,175$, 187; and relationship, connection between 175-176; social identity $11,83,94,169-171,177$, 179, 184, 193, 194, 196, 197, 204, 210; sociocultural identity 65 ; unrelated thought with relational thinking, overcoming 176-181
Identity and Control (White) 174

Identity and Difference (Heidegger) 180

IIX see Impact Investment Exchange (IIX)

immanent transcendence 218

Impact Investment Exchange (IIX) 42

impaired reflexivity 202

independent reflexivity 202

indeterminacy $1,5,221$

indirect volunteer 131

individual-in-relation 162, 206

individualism $58,89,132,151$; institutionalised 9, 22, 26; methodological $5,17,18,134,136,161$; moral 120; statist 219

industrial revolution 4.035

inequality $4,16,22,51,57,74,133,134$, $139,152,158$. see also equality

information and communication technologies (ICTs) 8, 26, 94, 120, 161, 188, 199, 201, 202, 213, 217

Information Society (Beniger) 2

institutionalised individualism 9, 22, 26

Internet 65, 102, 105, 119, 120, 156, 161, 196, 199, 227; relation 92

involuntary creation of poverty $144-145$

I-You relation 101

KIVA project 42

Knowledge Society 2

late modernity (late modern) 2, 25, 30, $31,83,108,120,151,156$

Lebensphilosophie (Simmel) 5

Letter on Humanism (Heidegger) 179 lex mercatoria 137

lib/lab dilemma 3, 5-6, 8-10; freedom vs control 15-34; good life 152, 155-158, 161, 165; market/state binary code, overcoming 35-50; modernity as $15-17$; morality of social relations 158,161 ; relational logic of happiness 156,157 ; social morality $113-116,119$, 121, 123, 128, 129; societal morphogenesis 101,107

linear causality 10, 130, 135

logic of opportunity, globalisation and $154-155$

mafia relation 159

market: as a black box 136-137; civilisation 145-146; outcomes 130-133; relation 92 , 136; rules 135 , 
138; see also market/state binary code, overcoming

market/state binary code, overcoming 35-50; civil society 42-46; Darwinian evolution 38-39; finalism 40-42; recurrent system 35-36; relational state $46-50$

merchandise acquisition, produced in human rights violation 144

metanarratives: condition of incredulity 6-7

meta-reflexivity 59, 64, 67, 122, 125, 138, 158, 183, 202, 205

methodological individualism 5, 17, 18, 134, 136, 161

modernity: civil society and $42-46$; definition of 2-3; features of 45; as lib/lab dilemma 15-17

modern relationality $85,87-90$

molecule: definition of 92; social 9, 73, 79, 81-95, 101, 103-105, 108, 109, 174, 227

moral criteria, for imputing responsibility $138-143$

moral good, relational character of 163-164

moral individualism 120

morality 38, 61, 153-156, 164-166; artificial 200; collective 153; as emergence of subjectivity 128 ; eudemonic 161-163; liberal 163; relational 9, 120, 156; socialist 153 ; social morality, morphogenesis of 111-127; of social networks 10, 115, 130-146, 159; of social relations $158-163$; as systemic emergence 127; of welfare 120-124; Western 9, 152

morphogenesis 2, 6, 33, 49, 58, 71, 72, 76, 79, 81-83, 85, 88, 92, 96, 108-110, 154, 156, 157, 161, 165, 168, 186, 187, 194, 195; adaptive 207; bound 124; cultural morphogenesis 2, 11; relational 125 ; relationally steered 208; of social morality 111-129; social see social morphogenesis; societal, as surplus of relational forms 101-107; turbulent 207; unbound 125 multiculturalism 115, 189 MYC4, 42

negative feedback $79,116-118$ neighbourhood relation 92 neo-communitarians 24 neoliberals 17, 24

neo-relationists $24-25$

networks, definition of 56n7

nihilism: cultural nihilism 216

nomos-building 118, 128

"Nothingness" (the absence of existence) 215

objectifying gaze 51

observing vs gazing $58-59$

ODG systems 48, 208-209

OMC see open method of coordination (OMC)

ontology 4, 5, 7, 8, 11, 49, 59, 65, 67, 74, $91,126,127,132,140,167,175,179$, 180, 193, 198, 201, 210, 224-227; deep 53; flat (single-level) 55, 57; monovalent 53; social 9, 52, 53, 55, 58, 61, 200, 217, 218, 220-226, 229-230; turbulent 53

open method of coordination (OMC) $122 \mathrm{n} 2$

partners in a couple, relation between 78

personal identity $64,129,167,169,171$, $174,175,178,179,186,188,189,225$; in morphogenetic process 186-188 personalisation 49

personal reflexivity (internal conversation) 6, 7, 11, 64-67, 97, 139, 162, 169, 172, 177, 186-188, 197, 199, 229; types of 64

pluralism 83

polycontexturality 206

positive feedback $79,80,91,116-118$

positivistic functionalism 17-18

postmodernity (postmodern) 2, 5, 6, 16, $17,24,25,30,33,71,83,120,155,165$, 178,188

post-truth politics 216

poverty $38,40,46,50,131-134,139,141$, 158 ; involuntary creation of $144-145$; trap 37, 159

processual (interactional and transactional) order 55, 56

professional relation 92

prosociality (prosocial) 25, 41, 103, 104, 107

pure relation $87,108,165$

quantum physics 57

quasi-social relationships 198 
rational choice theory 136

reciprocal action 5, 33, 55, 60, 74, 77, 79,

138, 160, 177, 198

reciprocation 119

redistributive economies 146

refero $29-33,65,74,75,80,98,167,175$, $180,188,225$

reflecting team $78,79 \mathrm{n} 2$

reflectivity (reflective) 10, 40, 54, 112, 151, 201, 202; general definition

$63 \mathrm{n} 3$; reflective mind 214-215; selfreflectivity 214 ; systemic reflectivity 67 ; system reflectivity 97

Reflexive modernisation (Beck,

Giddens, and Lash) 2

reflexive rationality 39,133

reflexive systems 151

reflexive truth 39,133

reflexivity (reflexive): autonomous

reflexivity $64,122,124,143,183$;

communicative reflexivity 64,122 ,

124,183 ; conformist reflexivity

201; critical reflexivity 202; general

definition 63n3; impaired reflexivity

202 ; independent reflexivity 202 ;

meta-reflexivity 59, 64, 67, 122, 125 ,

138, 158, 183, 202, 205; personal

reflexivity (internal conversation)

6, 7, 11, 64-67, 97, 139, 162, 169, 172,

177, 186-188, 197, 199, 229; relational

(or social) reflexivity $6,7,11,49,59$,

63-67, 79, 97, 139, 142, 143, 146, 162,

$168-173,175,177,178,182,186-189$,

198, 201, 213, 229; system reflexivity 7

rejection of risks, from bankruptcy of countries in financial crisis 144

relation(ship): agency-structure 6; as an emergent 5, 74-76; capitalist relation 92, 93; doctor-patient relationship 78; employer-employee relationship 78; enigma of the relation 218-220, 228 ; family relation $85,92,171,177$; feudal relation 92 ; founding relation $91 \mathrm{n} 8,119,180$; friendship relation 78 , 92, 160-161; as generative mechanism 79-82; and identity, connection between 175-176; Internet relation 92; I-You relation 101; latent, with a reality $61-63$; in logical sense $59-60$; mafia relation 159; physical, with the natural world 60 ; with the practical world 60-61; quasi-social relationships 198; with the social world 61; vital relationship 180, 181, 225; see

also individual entries

relational approaches $\mathbf{2 2 2}$

relational character of the moral good $163-164$

relational constitution $11,49,93,111$,

163, 167, 197, 200, 227, 229

relational enterprise 105

relational essentialism 180

relational ethics $138-143,157$

relational evils $10,11,77,91,98,112$, 137-139, 141, 157-159, 161, 175, 195, 205, 209, 210, 224

relational feedback $6,79,80,91,117$, $125,180,184,186$

relational gaze 7, 9, 51-67; first-person perspective of 66; second-person perspective of 66-67; third-person perspective of 67

relational goods $10-11,41,42,44$, 46-48, 50, 66, 77, 90, 91, 96-98, 100, 101, 104, 105-107, 110, 112, 118, 122 , $126,137-141,145,149,157,159-162$, 166, 195, 201, 205, 208-211, 224, 228,229 ; emergence of new social subjects generating 167-189; human identity vis-à-vis post-/trans-human 188-189; identity and relationship 167-176; recognition of identity 181-182; social in relational subject 182-186; through social capital, generation of 105; unrelated thought with relational thinking, overcoming 176-181

relational humanism 54, 228

relational identity 169, 171-173, 175, 187

relational inclusion 7, 119

relational logic of happiness 155-157

relationally steered morphogenesis

107-108, 208

relational morality $9,120,156$

relational morphogenesis 125

relational outcomes 48-49

relational reality $67,168,176$

relational reason 110

relational (or social) reflexivity $6,7,11$, 49, 59, 63-67, 79, 97, 139, 142, 143, $146,162,168-173,175,177,178,182$, 186-189, 198, 201, 213, 229; sociological gaze and 63-67

relational society $1,2,7,11,12,31$, 33, 51, 67, 188, 229, 230; economic logic of 39,41 ; as morphogenetic 
configuration 71-110; relational state, need for 46-50

relational sociology $1,4,5,9,11,53,54$, 57, 64, 72, 73, 88, 90, 144, 165, 174, $175,179,186,210,213,220,223,224$, 226, 227; constructivist 7; critical 7; realist 7

relational state $46-50,119,122,123$

relational structural order 55,56

relational subject $10-12,33,36,49$, $80,91,93,97,110,118,119,124,127$, 136, 145, 155, 181-189, 197, 202, 205; personal and collective identity in morphogenetic process $186-188$; role in 137-139; social in 182-186; social networks 140-143

relational thinking $8,12,50,57,103$; dissolution of humanity with, confronting 227-230; unrelated thought with, overcoming 176-181 relational tourism 105

relational turn $5,6,123$

relational value $99-100$

relational work 46, 48, 77, 158, 209

relationist approaches $\mathbf{2 2 2}$

relationist gaze 53

religio $29-33,65,74,75,98,167,174$, $180,188,225$

reverse discrimination 166

right of citizenship 121

Rise of the Network Society, The

(Castells) 2

Risk Society (Beck) 2

SAC (structure-agency-culture) scheme 186

SASIX (South African Social

Investment Exchange) 42

scientific gaze 51-54

self-reflectivity 214

Silicon Valley multinationals 95

social capital 6, 51, 95-97, 100, 105-107,

$118,160,161$

social causality, as relational causality 145

social cohesion 121, 155

social cooperation, instructive example of 103-107

social dumping 143-144

social form of humans $226-227$

social identity 11, 83, 94, 169-171, 177,

179, 184, 193, 194, 196, 197, 204, 210

social integration vs system integration $23,28-29$ social market economies 146

social molecules 9, 73, 79, 81-95, 101, 103-105, 108, 109, 174, 227

social morality, morphogenesis of: agency-structure relationship 111-112; alternative view 112-115; classic paradigms, obsolescence of 115-116; relationality in generating morals, importance of 117-120; societal moral fabric, in era of globalisation 124-127

social morphogenesis: chaotic (highly unbound) social morphogenesis 107-108; highly constrained (highly bound) social morphogenesis 107 ; liquid 109; realisation of 107-109; relationally steered morphogenesis 107-108

social networks: capitalism of 95 ; example 143-145; market, as a black box 136-137; market outcomes in 130-133; moral criteria, for imputing responsibility 138-143; morality of 10, 115, 130-146, 159; prevailing explanations of causality, questioning 133-136

social ontology $9,52,53,55,58,61,200$, 217, 218, 220-226, 229-230

social relations 1,5 ; definition of 65 , 98; as molecule of the social 82-95; morality of 158-163; non-fungibility of 90; semantics of 74-75; social morphogenesis, realisation of 107-109; social value of 95-101; structure of 86 ; theory of 73-79; as transactions 75

Social Stock Exchange Asia (SSXA) 42 Social Stock Exchange Ltd. 42

social subject $8,33,36,114,119$, 167-189, 202

societal citizenship 33

societal evolution, scenarios of 212-218

societal moral fabric, in era of globalisation 124-127

societal morphogenesis, as surplus of relational forms 101-107

society: bureaucratisation of 6; civil 23 , 26, 31, 33, 37, 38, 42-47, 49, 50, 101, 103, 118, 119, 121, 146, 149, 153, 177, 178; definition of 71-73; morphogenesis of 71-110; as operating system 207; relational $1,2,7,11,12,31,33,39,41$, 46-51, 67, 71-110, 188, 229, 230; see also individual entries 
sociocultural identity 65

sociological gaze, relational reflexivity and $63-67$

solidarity $25-27,30,32,41,49,59,72$, $81,102,109,122,154,159,162,163$, 189, 228; primary 121; secondary 121 SSXA see Social Stock Exchange Asia (SSXA)

strategic action 101

structural effects 95-101

structural neo-functionalism 25, 38

structural relational constitution 229

subjectivising gaze 51, 58, 67

subject-object relation 202

substantialist approaches 222

sui generis 10, 12, 26, 72, 91, 92, 96, 109, $111,119,123,140,153,164,184,186$, 224-227

superordinate value of action 83

suprafunctional non-equivalence 88

sustainable development 40-41

Swedish Theory of Love, The 219

synergic antithesis 16, 19, 20, 22, 24

synergy $8,16-21,23,24,41,110,129$, 196

systemic functionalism 151

systemic reflectivity 67

system reflexivity 7

Tausch (exchange) 3-5

teacher-pupil relation 92

Technological-Gnosis (Tech-Gnosis) 11, 194, 210

theory of social relations $73-79$;

composition 75-76; form 76-79

therapeutic relation $49,77,78,92$

third sector relation 92

Third Way 3, 114, 121

transactional sociology 54, 186

transcendence 86, 181, 189, 217, 220, 221, 224, 224n4; human 212, 216; immanent transcendence 218;

transcendent transcendence 218

transcendent transcendence 218

trans-modernity (trans-modern) 2, 9, 226

tribal relation 92

turbulent morphogenesis 207

turbulent ontology 53

unbound morphogenesis 125

unemployment 48, 121, 131, 133, 139,

$141,143,144,208$

unintentional structural effects 132

unitas multiplex 206

unrelated thought with relational

thinking, overcoming 176-181

use value $99-100$

valorisation $9,42,100,101,103$

value of dignity 100

Vergesellschaftung (socialisation) 3, 23, 63,173

vitalism 216

vital relationship 180, 181, 225

Wechselwirkung (reciprocity effect) 3, 4, 75

We-in-relation 110

We-relation 66, 97, 110, 124, 145, 155 , 159, 160, 162, 167, 182-184, 188, 197

Wertrationalität (value rationality) 189

Western political democracy 118

Western rationalism 216

world system (or globalisation) 1, 8, 22, 26, 36-37, 39, 49, 50, 128, 132, 146; era, societal moral fabric in 124-127; logic of opportunity $154-155$

WTO 95

Zweckrationalität (instrumental rationality) 189 